\title{
Lead Selenide Quantum Dot Luminescent Solar Concentrators
}

\author{
A Dissertation \\ Presented to the advisory committee \\ at the Department of Electrical and Computer Engineering \\ University of Virginia \\ in partial fulfillment \\ of the requirements for the degree \\ Doctor of Philosophy (Electrical and Computer Engineering) \\ by \\ Dennis L. Waldron \\ B.Sc., Electrical and Computer Engineering, Lafayette College, 2010
}

May 2015 


\section{Approval Sheet}

This dissertation is submitted in partial fulfillment of the requirements for the degree of Doctor of Philosophy (Electrical and Computer Engineering)

Dennis L. Waldron

This dissertation has been read and approved by the Examining Committee:

Professor Mool C. Gupta, Adviser

Professor Archie Holmes, Committee Chair

Professor Joshua Choi

Professor Tatiana Globus

Dr. Joseph M. Zawodny, NASA Langley Research Center

Accepted for the School of Engineering and Applied Science:

Professor James H. Aylor, Dean, School of Engineering and Applied Science May 2015 


\section{Abstract}

Worldwide photovoltaic energy generation capacity is expected to double or triple 2013 levels by 2018 [1]. There is thus considerable motivation to reduce the cost of photovoltaic energy generation. Luminescent solar concentrators (LSCs) have the potential to reduce the price per Watt of solar power. LSCs operate by efficiently capturing sunlight over a broad spectrum using a fluorescent molecule such as dyes or quantum dots. The emitted fluorescent light is guided in a polymer matrix to the edges where a photovoltaic cell is attached for optical to electric power conversion. So, fluorescent dyes or quantum dots play an important role for efficient solar power conversion.

Here, functionalized, high quantum yield ( $\geq 70 \%$ ) lead selenide quantum dots (PbSe QDs) are investigated for use in LSCs to replace dyes as the luminescent molecule. PbSe QDs have a much wider absorption spectrum than dyes, and so are able to absorb more incident solar radiation. Individual optical losses in LSCs are characterized. These losses and routes to LSC optimization are investigated with a custom ray tracing model.

A method of incorporating QDs into an AB9093 epoxy or a poly(lauryl methacrylate-coethylene glycol dimethacrylate) polymer matrix is presented, as well as a discussion of other matrix candidates. The resulting QD nanocomposite optical absorption and fluorescence properties at room temperature were studied, and their properties in an AB9093 matrix were characterized as a function of temperature from $0^{\circ} \mathrm{C}$ to $80^{\circ} \mathrm{C}$ and compared to QDs in a toluene solution. A decrease in fluorescence intensity was found as temperature increased.

A nanocomposite LSC was fabricated and mated with a monocrystalline silicon photovoltaic cell. The resulting system had a power conversion efficiency (PCE) of $4.93 \%$ measured under one sun broadband illumination. This is the highest known broadband PCE of any LSC system using either silicon photovoltaic cells or QDs of any kind. 


\section{Acknowledgments}

I would like to thank my advisor, Dr. Mool C. Gupta for his help and guidance in pursuing my Ph.D. under the NASA Langley program at the National Institute of Aerospace, and NASA mentor, Dr. Joseph Zawodny, with whom my conversations are always thought provoking.

This work would not have been possible without the ongoing support of my collaborators at the University of Rochester Department of Chemistry, including, but not limited to Dr. Todd Krauss and graduate student Amanda Preske.

I made many great friends during my time in graduate school, the vast majority of which I will remember very fondly. Two, Craig Ungaro and Duncan McGillivray, deserve special mention for all of their help and for listening to my various rants and inconsequentialities.

Finally, I thank my wife and family, to whom there are not enough thanks to be given.

This work was supported by the NASA Langley Professor program and NSF I/UCRC. 


\section{Contents}

$\begin{array}{ll}\text { Abstract } & \text { ii }\end{array}$

$\begin{array}{ll}\text { Acknowledgments } & \text { iii }\end{array}$

List of Tables $\quad$ vii

List of Figures viii

List of Symbols and Abbreviations $\quad$ xi

1 Introduction $\quad 1$

1.1 Luminescent Solar Concentrators . . . . . . . . . . . . . . . . . 1

1.1.1 Operating Principle . . . . . . . . . . . . . . . . . 1

1.1.2 Advantages over Geometric Concentrators . . . . . . . . . . . . . . . 2

1.1 .3 Ideal Matrix Material . . . . . . . . . . . . . . . . . . . 3

1.1 .4 Luminophores . . . . . . . . . . . . . . . . . . . . . . . . . . . . . . . . . .

1.1.5 Different LSC Architectures and Advancements . . . . . . . . . . . . 5

1.2 State of the Art . . . . . . . . . . . . . . . . . 6

1.3 Other Applications of QD Nanocomposites . . . . . . . . . . . . 7

1.4 Problem Statement . . . . . . . . . . . . . . . . . . . . 8

2 Fundamentals $\quad 9$

2.1 Photovoltaic Cells . . . . . . . . . . . . . . . . . . . . . . 9

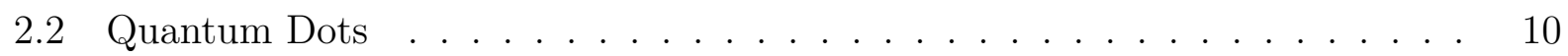

2.2.1 Physical Structure . . . . . . . . . . . . . . . . . . . 11

2.2.2 Exciton Bohr Radius . . . . . . . . . . . . . . . . . . 12

2.2.3 Electronic and Optical Spectra . . . . . . . . . . . . . . 13

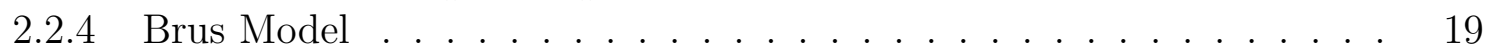

2.2.5 Effect of Temperature . . . . . . . . . . . . . . . . 22

2.3 Geometric Solar Concentrators . . . . . . . . . . . . . . . . . 25

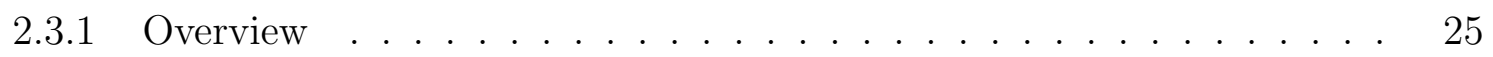

2.3.2 Maximum Concentration Factor . . . . . . . . . . . . . . . . 26

2.4 Luminescent Solar Concentrators . . . . . . . . . . . . . . . . . . . . . . . . . . . . . . . . . . 27

2.4.1 LSC Optical Losses . . . . . . . . . . . . . . . . . . . . . . . . . . . . . . . . . . . .

2.4 .2 LSC Concentration Limits . . . . . . . . . . . . . . . . . . . 35

2.5 Polymerization of Matrix Material . . . . . . . . . . . . . . . . 37

3 LSC Modeling $\quad 41$

3.1 Background . . . . . . . . . . . . . . . . . . . . . . 41

3.2 Baseline Simulation . . . . . . . . . . . . . . . . . . . . . . . . . . . . . . . . . . . . . . .

3.3 Model Verification and Limitations . . . . . . . . . . . . . . . . . . . 53

3.4 Effect of Changing Individual LSC Parameters . . . . . . . . . . . . . . 57 
3.5 Ideal Case . . . . . . . . . . . . . . . . . . . . . . . . . 63

3.6 Conclusion . . . . . . . . . . . . . . . . . . . . . 65

4 Experiment $\quad 66$

4.1 QD Synthesis . . . . . . . . . . . . . . . . . . 6 66

4.2 Matrix Preparation and QD Incorporation . . . . . . . . . . . 67

4.2 .1 Sol-gels . . . . . . . . . . . . . . . . . . . . . . 67

4.2 .2 Cast PMMA . . . . . . . . . . . . . . . . . . . . . . . . 67

4.2 .3 Cured PMMA . . . . . . . . . . . . . . . . . . . . 67

4.2 .4 Two part epoxies . . . . . . . . . . . . . . . . . . . . . . 68

$4.2 .5 \quad$ AB9093 Epoxy . . . . . . . . . . . . . . . . . . . . 68

$4.2 .6 \quad \mathrm{P}($ LMA-co-EGDMA $) \ldots \ldots \ldots . \ldots \ldots$

4.2 .7 UV Curing . . . . . . . . . . . . . . . . . . . . . . . . . . . . . . . . . . . . . 69

4.3 Optical Characterization . . . . . . . . . . . . . . . . . 70

4.4 Morphology Characterization by SEM \& TEM . . . . . . . . . . . . . . 71

4.5 Temperature Control Setup and Samples . . . . . . . . . . . . . . . 72

4.6 LSC PV System Fabrication . . . . . . . . . . . . . . . . . . . 73

4.7 Solar Simulation Setup . . . . . . . . . . . . . . . . . . . . . . 74

$\begin{array}{llr}5 & \text { QD Nanocomposite Characterization } & \mathbf{7 6}\end{array}$

5.1 Matrix Characterization . . . . . . . . . . . . . . . . . . . 77

5.2 Optical Absorption Study . . . . . . . . . . . . . . . . . . . . 78

5.3 QD Fluorescence Study . . . . . . . . . . . . . . . . . . . . . . . . . . . 79

5.4 Conclusion . . . . . . . . . . . . . . . . . . . . . . 81

6 Temperature Dependent Effects $\quad 83$

6.1 Optical Absorption Study . . . . . . . . . . . . . . . . . . . . 83

6.2 QD Fluorescence Study . . . . . . . . . . . . . . . . . . . . . . . 85

6.3 Conclusion . . . . . . . . . . . . . . . . . . . . . . 88

$\begin{array}{lll}7 & \text { Luminescent Solar Concentrators } & 91\end{array}$

7.1 Initial Tests . . . . . . . . . . . . . . . . . . . . . . . . . 91

7.2 Best QD LSC . . . . . . . . . . . . . . . . . . . . . . . . . . 94

7.3 Conclusion . . . . . . . . . . . . . . . . . . . 96

8 Conclusions \& Future Work $\quad 98$

8.1 Conclusions . . . . . . . . . . . . . . . . . . . . . . . 98

8.2 Future Work . . . . . . . . . . . . . . . . . . . . . . . 100

$\begin{array}{lr}\text { Bibliography } & 103\end{array}$

$\begin{array}{ll}\text { Appendices } & 117\end{array}$

$\begin{array}{ll}\text { A List of Publications } & 117\end{array}$ 
B Ray Tracing Simulation MATLAB Code $\quad 118$

B.1 batch.m . . . . . . . . . . . . . . . . . . . . . . 119

B.2 Rtrace.m . . . . . . . . . . . . . . . . . . . . . 124

B.3 fresTMM.m . . . . . . . . . . . . . . . . . . . 135 


\section{List of Tables}

3.1 Simulated final photon location probabilities for a "default" LSC . . . . . . 48

3.2 Simulated losses for a "default" LSC . . . . . . . . . . . . . . . . . 49

6.1 Summary of temperature dependent measured parameters. . . . . . . . . . 90

7.1 AB9093 QD LSC parameters, initial testing . . . . . . . . . . . . 93

$7.2 \mathrm{P}(\mathrm{LMA}-\mathrm{co}-\mathrm{EGDMA})$ QD LSC parameters, initial testing . . . . . . . . 93

7.3 Measured parameters for the best performing LSC system . . . . . . . . . 95 


\section{List of Figures}

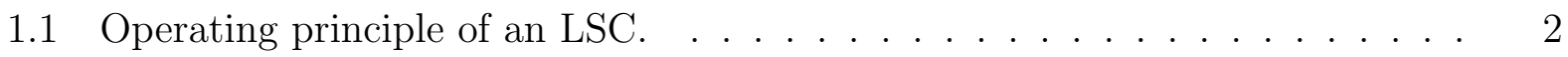

1.2 Comparison of the absorption spectra of various luminophores. . . . . . . . 5

1.3 Various alternative LSC architectures. . . . . . . . . . . . . 6

2.1 PV cell diagram . . . . . . . . . . . . . . . . . . . . . . . . . . 10

2.2 QDs displaying size-dependent fluorescence color . . . . . . . . . . . . . . 11

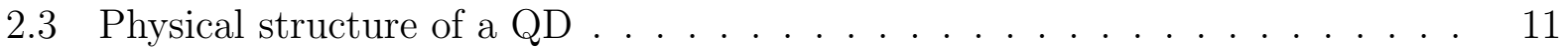

2.4 Energy band diagram for various crystal sizes . . . . . . . . . . . . . . . 13

2.5 A simple model of semiconductor optical absorption . . . . . . . . . . . . . 14

2.6 Fluorescence emission spectra shape . . . . . . . . . . . . . . . . . . . . . 16

2.7 Stokes shift configurational coordinate diagram $\ldots \ldots \ldots \ldots$

2.8 FRET diagram $\ldots \ldots \ldots \ldots \ldots \ldots$

2.9 Brus model prediction for PbSe QDs. . . . . . . . . . . . . . . . . . . . 22

2.10 Geometric concentrator diagram . . . . . . . . . . . . . . . . . 26

2.11 Geometric concentration factor $\ldots \ldots \ldots \ldots 27$

2.12 Optical losses in an LSC . . . . . . . . . . . . . . . . . . . . . 28

2.13 Escape cone diagram . . . . . . . . . . . . . . . . . . . . . . . . . 29

2.14 Effect of matrix material index on optical losses. . . . . . . . . . . . . 30

2.15 Self absorption in a luminophore . . . . . . . . . . . . . . . . 30

2.16 TIR loss diagram $\ldots \ldots \ldots \ldots$

2.17 PMMA near IR absorption . . . . . . . . . . . . . . . . . . 33

2.18 Maximum concentration factor for LSCs. . . . . . . . . . . . . . . . 37

2.19 Maximum concentration factor for LSCs with temperature. . . . . . . . . 37

2.20 Matrix polymerization example . . . . . . . . . . . . . . . . . 38 
3.1 Simulation matrix attenuation coefficients . . . . . . . . . . . 43

3.2 Simulation QD spectra . . . . . . . . . . . . . . . . 43

3.3 Simulation source spectra f. . . . . . . . . . . . . . 43

3.4 Simulation refractive indices . . . . . . . . . . . . . . . 43

3.5 Simulation emission CDF . . . . . . . . . . . . . . . 46

3.6 Simulation PV cell dielectric stack reflectance . . . . . . . . . . . . . 46

3.7 Selected simulated LSC losses, default case . . . . . . . . . . . . . . 50

$3.8 \eta_{\text {opt }}$ and PCE, default case . . . . . . . . . . . . . . . 50

3.9 Simulated LSC output spectrum, default case . . . . . . . . . . . . 51

3.10 Simulated LSC photon output angles, default case . . . . . . . . . . . . 52

3.11 Simulated LSC photon travel distance, default case . . . . . . . . . . 53

3.12 Combined dye spectra . . . . . . . . . . . . . . . . . . 54

$3.13 \eta_{\text {opt }}$ and PCE, combined dye . . . . . . . . . . . . . . . 55

3.14 Simulated LSC losses, combined dye . . . . . . . . . . . . . . . 55

3.15 LSC bonding flaws . . . . . . . . . . . . . . . . . . 56

3.16 Simulated LSC output spectrum, combined dye . . . . . . . . . . . . 57

3.17 Simulated LSC $\eta_{\text {opt }}$ for various QY . . . . . . . . . . . . . . . . 58

3.18 Simulated LSC $\eta_{\text {opt }}$ for various bottom mirror types . . . . . . . . . . . . 59

3.19 Reflectivity before and after ARC optimization . . . . . . . . . . . . . 60

3.20 Simulated LSC $\eta_{\text {opt }}$ for various PV cell ARC thicknesses . . . . . . . . . . . 60

3.21 Simulated LSC $\eta_{\text {opt }}$ for various matrix absorption values . . . . . . . . . 60

3.22 Simulated LSC $\eta_{\text {opt }}$ for various mirror reflectivities . . . . . . . . . . . . . 61

3.23 Simulated LSC $\eta_{\mathrm{opt}}$ for various matrix refractive indices . . . . . . . . . . 62

3.24 Simulated LSC $\eta_{\text {opt }}$ for various TIR loss fraction per bounce . . . . . . . . . 62

3.25 Simulated LSC PCE with various luminophore spectra . . . . . . . . . . 63

3.26 Simulated losses in an ideal system . . . . . . . . . . . . . . . . 64

3.27 Simulated LSC $\eta_{\text {opt }}$ and PCE in an ideal system . . . . . . . . . . . . . 64 
4.1 Mold for UV cured polymer castings. . . . . . . . . . . . . . . . 70

4.2 Setup for measuring QD fluorescence spectrum. . . . . . . . . . . . . 71

4.3 Setup for measuring scattered light. . . . . . . . . . . . . . . . 71

4.4 Temperature control experimental setup. . . . . . . . . . . . . . . 72

4.5 Setup for measuring the I-V curve of the LSC PV system. . . . . . . . . 74

4.6 Broadband halogen lamp spectrum compared to sunlight. . . . . . . . . . . 74

5.1 Attenuation coefficient of various clear polymers . . . . . . . . . 77

5.2 Optical power scattered in various materials $\ldots \ldots \ldots 77$

5.3 QD optical absorption in various materials . . . . . . . . . . . 79

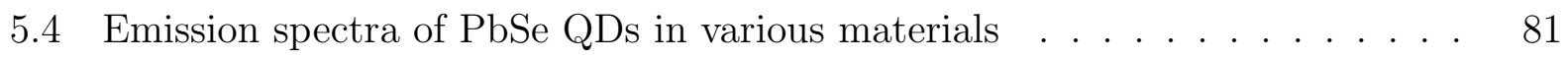

5.5 Time resolved photoluminescence of QDs in toluene and AB9093. . . . . . 81

6.1 Absorption spectra at various temperatures for QDs in AB9093 and toluene 84

6.2 QD optical absorption at the 1-s peak as a function of temperature $\ldots . .85$

6.3 Wavelength position of the 1-s peak as a function of temperature . . . . . . 85

6.4 Fluorescence spectra of QDs and Red 305 dye . . . . . . . . . . . . 86

6.5 QD fluorescence intensity as a function of temperature $\ldots \ldots \ldots$

6.6 QD fluorescence peak wavelength shift as a function of temperature . . . . 88

6.7 QD emission FWHM of the fluorescence peak as a function of temperature . 89

7.1 Photos of a fabricated LSC . . . . . . . . . . . . . . . . . . . . 92

7.2 Best P(LMA-co-EGDMA) LSC I-V curve . . . . . . . . . . . . . . 95

7.3 QD absorption and fluorescence in the LSC $\ldots \ldots \ldots . \ldots . \ldots 95$ 


\title{
List of Symbols and Abbreviations
}

\author{
$\alpha \quad$ absorption coefficient \\ $\gamma \quad$ surface tension \\ $\gamma_{\mathrm{AC}} \quad$ exciton-acoustic phonon scattering coefficient \\ $\Gamma \quad$ quantum dot emission linewidth \\ $\Gamma_{\text {inh }} \quad$ quantum dot emission inhomogeneous broadening \\ $\Gamma_{\mathrm{LO}} \quad$ exciton-longitudinal optical phonon coupling strength \\ $\delta_{\mathrm{CH}} \quad$ optical absorption band of the bending vibrational mode of the carbon- \\ hydrogen bond \\ $\epsilon$ \\ bulk dielectric constant \\ $\epsilon_{0}$ \\ vacuum permittivity, $8.854187 \times 10^{12} \mathrm{~F} \cdot \mathrm{m}^{-1}$ \\ $\eta_{\mathrm{opt}}$ \\ optical efficiency \\ $\theta$ \\ in simulation: photon polar angle, a.k.a. deflection or zenith angle, \\ [0 to $\pi], 0={ }^{+} z$ direction (up) \\ $\theta_{\mathrm{c}}$ \\ critical angle for TIR \\ $\kappa$ \\ extinction coefficient \\ $\lambda$ \\ wavelength \\ $\lambda_{\mathrm{g}}$ \\ wavelength of the band gap energy \\ $\nu$ \\ frequency, $\mathrm{Hz}$ \\ $\nu_{\mathrm{CH}}$ \\ harmonic of the optical absorption band of the stretching vibrational \\ mode of the carbon-hydrogen \\ $\xi$ \\ random number in the range [0 to 1$]$ with a uniform distribution \\ $\pi$ \\ constant, ratio of a circle's circumference to diameter, 3.14159265359 \\ $\sigma$ \\ a measure of surface roughness: peak height of a circle segment \\ protruding above the surface plane
}




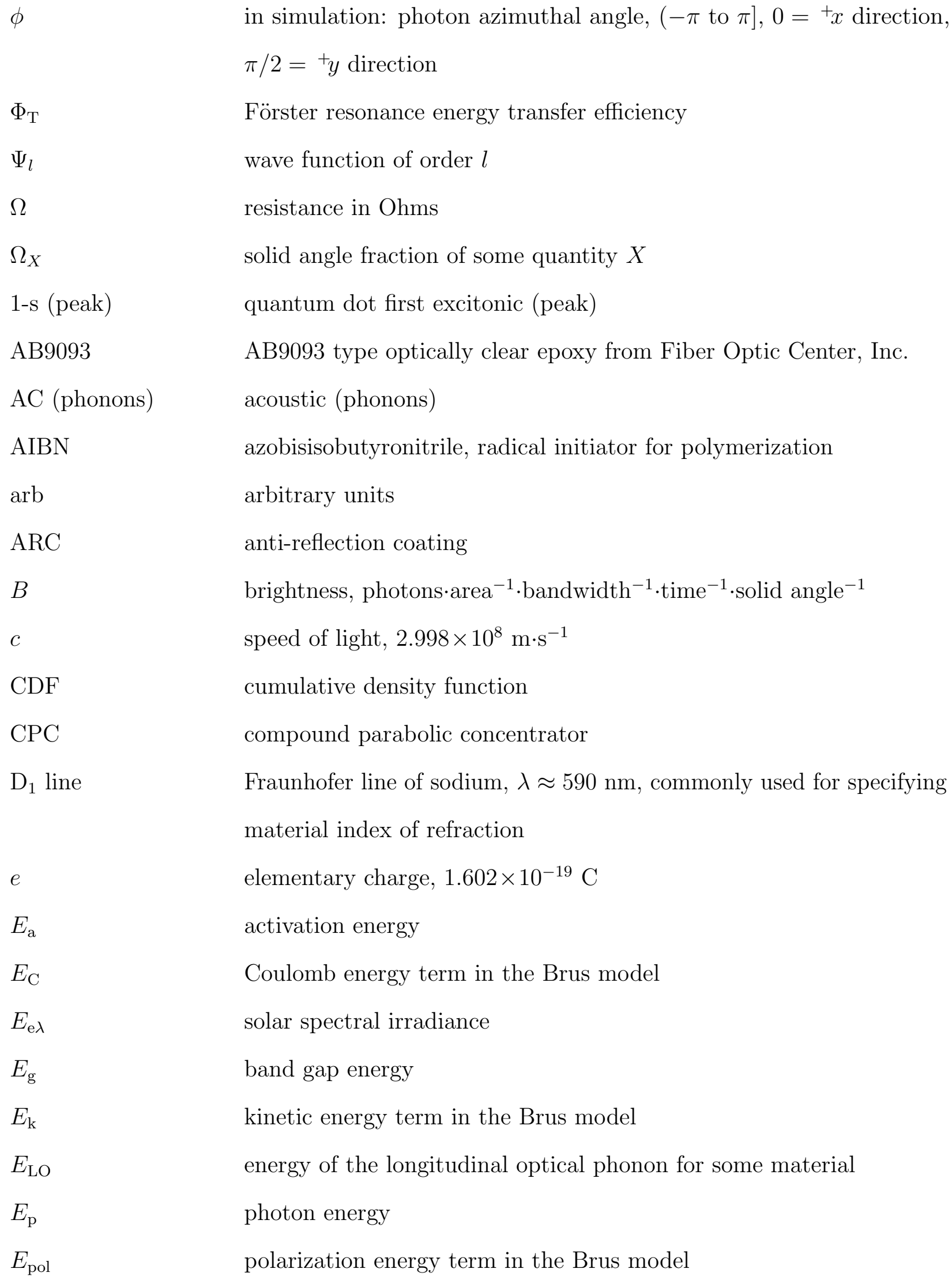




\begin{tabular}{|c|c|}
\hline EGDMA & ethylene glycol dimethacrylate (monomer) \\
\hline EQE & external quantum efficiency \\
\hline$f$ & focal length \\
\hline$F_{\mathrm{EC}}$ & fraction of fluorescent photons captured by TIR in a cuboid matrix \\
\hline$F F$ & fill factor \\
\hline FRET & Förster resonance energy transfer \\
\hline FSR & front surface reflection \\
\hline FWHM & full width at half maximum \\
\hline G & $\begin{array}{l}\text { geometric gain, ratio of LSC collecting surface area to output edge } \\
\text { surface area }\end{array}$ \\
\hline$h$ & Planck's constant, $6.62606957 \times 10^{34} \mathrm{~J} \cdot \mathrm{s}$ or $4.135667516 \times 10^{15} \mathrm{eV} \cdot \mathrm{s}$ \\
\hline$\hbar$ & reduced Planck's constant, $\hbar=h / 2 \pi$ \\
\hline HOMO & highest occupied molecular orbital \\
\hline HQ & hydroquinone, polymerization inhibitor \\
\hline$I$ & intensity (e.g. fluorescence intensity) \\
\hline$I_{0}$ & saturation current density \\
\hline$I_{\mathrm{mpp}}$ & current at the maximum power point, Amps \\
\hline$I_{\mathrm{sc}}$ & short circuit current, Amps \\
\hline IR & infrared \\
\hline$k$ & wavenumber \\
\hline$k_{\mathrm{B}}$ & Boltzmann's constant, $1.3806488 \times 10^{23} \mathrm{~J} / \mathrm{K}$ or $8.6173324 \times 10^{5} \mathrm{eV} / \mathrm{K}$ \\
\hline$k_{\mathrm{e}}$ & Coulomb's constant, $8.987551 \times 10^{9} \mathrm{~N} \cdot \mathrm{m}^{-2} \cdot \mathrm{C}^{-2}$ \\
\hline$L_{\mathrm{b}}$ & loss per bounce inside of a waveguide from TIR loss \\
\hline $\mathrm{L}_{\mathrm{EC}}$ & escape cone loss from an isotropic emitter \\
\hline LMA & lauryl methacrylate (monomer) \\
\hline LO (phonons) & longitudinal optical (phonons) \\
\hline LSC & luminescent solar concentrator \\
\hline
\end{tabular}




\begin{tabular}{|c|c|}
\hline LUMO & lowest unoccupied molecular orbital \\
\hline$m$ & mass \\
\hline$m_{0}$ & rest mass of an electron, $9.10938291 \times 10^{-31} \mathrm{~kg}$ \\
\hline$m_{\mathrm{c}}^{*}$ & effective mass of a point charge \\
\hline$m_{\mathrm{e}}^{*}$ & effective mass of electrons in a semiconductor \\
\hline$m_{\mathrm{h}}^{*}$ & effective mass of holes in a semiconductor \\
\hline MEHQ & monomethyl ether of hydroquinone, polymerization inhibitor \\
\hline MMA & methyl methacrylate (monomer) \\
\hline$n$ & index of refraction \\
\hline$N$ & in simulation: relative QD concentration \\
\hline NREL & United States National Renewable Energy Laboratory \\
\hline$p$ & momentum \\
\hline$P_{\text {elec }}$ & electrical power, Watts \\
\hline$P_{\text {mpp }}$ & electrical power at maximum power point, Watts \\
\hline$P_{\mathrm{opt}}$ & optical power, Watts \\
\hline $\mathrm{PbSe}$ & lead selenide \\
\hline PCE & power conversion efficiency \\
\hline PID & proportional-integral-derivative \\
\hline P(LMA-co-EGDMA) & poly(lauryl methacrylate-co-ethylene glycol dimethacrylate) (co- \\
\hline & polymer) \\
\hline PMMA & poly(methyl methacrylate) (polymer) \\
\hline PV & photovoltaic \\
\hline$q$ & point charge magnitude, Coulombs \\
\hline$Q$ & configurational coordinate, a.k.a. nuclear coordinate \\
\hline QD & quantum dot \\
\hline QY & quantum yield \\
\hline
\end{tabular}




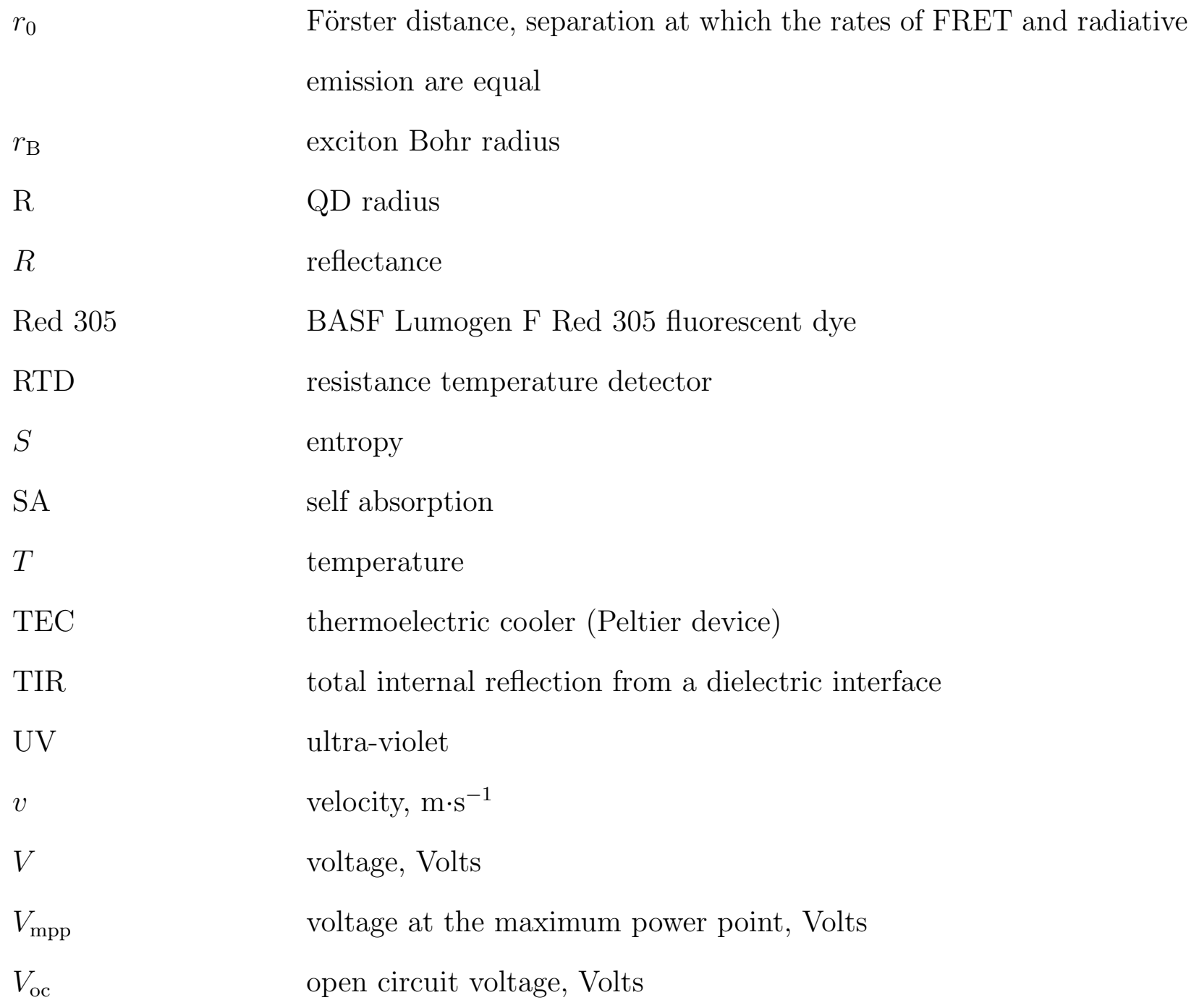




\section{Chapter 1: Introduction}

Worldwide installed capacity of solar energy generation has increased exponentially, totaling 138.9 GW by 2013, with 38.4 GW installed in 2013 alone; by 2018, worldwide capacity is

expected to double or triple [1]. Even so, solar energy is a vast largely untapped source of carbon-neutral energy with about $4.3 \times 10^{20} \mathrm{~J}$ of energy reaching the Earth's surface every hour, more energy than the $4.1 \times 10^{20} \mathrm{~J}$ used by the entire planet in the year of $2005[2]$.

Many installations rely on concentrators or at least solar trackers to boost capacity and reduce the price of generated electricity. These concentrators are mainly "geometric," using various mirrors, parabolic dishes, or lenses to guide and concentrate light on to the solar cell. Luminescent solar concentrators (LSCs), first proposed in the mid-1970's [3,4], instead use a luminescent material in a waveguiding configuration.

\subsection{Luminescent Solar Concentrators}

\subsubsection{Operating Principle}

The two primary LSC components are a waveguide which directs radiation to a PV (photovoltaic) cell and a luminophore, a molecule capable of absorbing a photon and emitting another at a longer wavelength. Additionally, the waveguide acts as a matrix for the luminophore. LSC operation is shown in figure 1.1. An incoming photon (blue line) enters the LSC from the top surface and is absorbed by a luminophore (red dot). The luminophore then emits a longer wavelength photon (green line), which is guided via total internal reflection to the solar cell (black) on the LSC edge. A diffuse reflector, with an air gap to preserve waveguiding, reflects any photons which make it through the LSC unabsorbed back at a high angle for greater absorption potential due to the longer photon path length. A specular mirror is put in place with no air gap on any edge to which a solar cell is not attached. This 


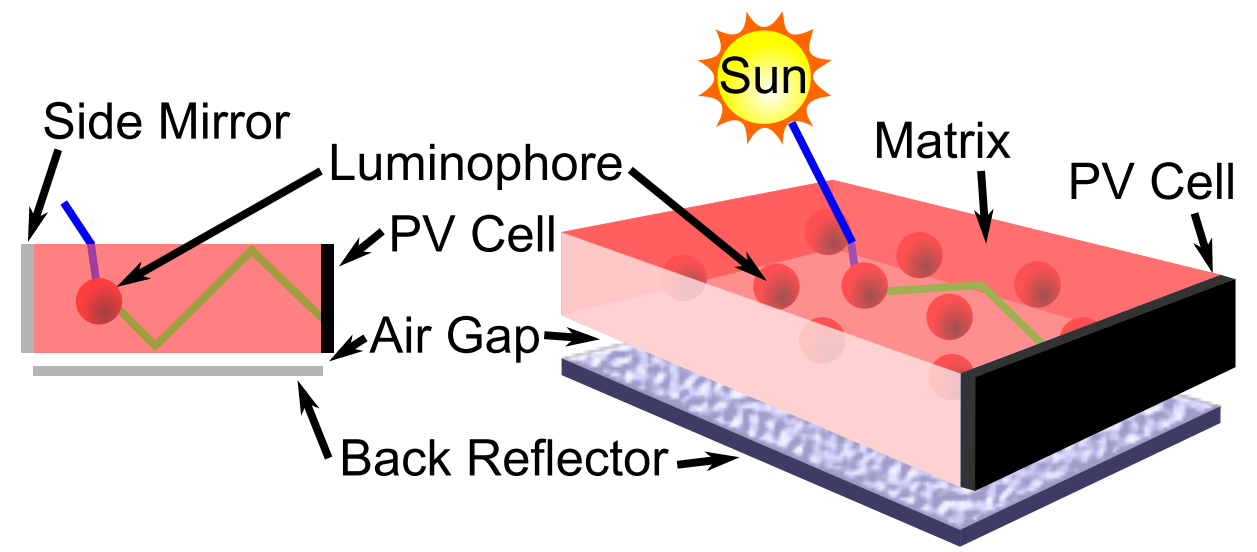

Figure 1.1: Operating principle of an LSC. Left: cross section view; Right: perspective view. A photon enters the LSC, gets absorbed by a luminophore, emitted at a wavelength ideal for the PV cell, and then guided via total internal reflection to a PV cell attached at the edge.

ensures that all light, whether it would have been reflected from the index change or not, is reflected back into the LSC on such an edge.

\subsubsection{Advantages over Geometric Concentrators}

LSCs have several advantages over other concentrator designs, making them an attractive alternative. For example, they drastically reduce the thermal load on the PV cell for a given optical concentration, eliminating the need for the PV cell to be cooled to avoid a reduction in PV cell efficiency. This is because emitted light is narrow band and matched closely to the PV cell band gap energy.

More importantly, LSCs can capture and guide direct, off-axis, and diffuse light. Thus they do not require expensive solar tracking mechanisms like geometric concentrators; an average one-axis tracker adds about $\$ 0.15$ per Watt in system costs, while a two-axis tracker adds about $\$ 0.45$ per Watt [5]. Even on a clear day near the equator, diffuse light accounts for at least $11.5 \%$ of light which reaches the earth's surface [6] and $60 \%$ or more at higher latitudes [7], most of which a geometric concentrator cannot capture. Since LSCs eliminate the need for trackers, systems can be placed much closer together without fear of shadowing neighboring systems. Partial shadowing by debris is also not an issue because the LSC ensures 
even illumination across the PV cell face, a result of the typically isotropic emission of the luminophores.

LSCs can be made with very low cost and mature manufacturing processes such as casting or even coating and roll-to-roll techniques. The manufactured pieces can then be used as architectural components such as windows or roofing, or as a coating on other pieces in addition to their use in a dedicated installation. So, there is considerable interest in fabricating high efficiency and low cost LSCs.

\subsubsection{Ideal Matrix Material}

The ideal matrix is perfectly transmissive in the ultraviolet (UV) through near-infrared (near-IR) region (wavelength $\lambda=360 \mathrm{~nm}$ to $1100 \mathrm{~nm}$ ) to match the silicon PV cell absorption spectrum. This allows all sunlight and fluorescent light which can be absorbed by a silicon PV cell to pass through the matrix. The matrix should also be non-scattering to promote waveguiding, as well as lightweight and strong. The matrix material's index of refraction $n$ is also an important consideration as it dictates waveguiding properties and optical losses, as discussed in section 2.4.1.

The matrix must be chemically compatible with the luminophore. Not only must the luminophore easily disperse into the matrix material, the luminophore's optical properties must not be destroyed upon incorporation into the matrix. Gallagher et al. highlight this in a study examining various polymer matrix materials for Cd-salt quantum dots (QDs) and find up to a $77 \%$ retention of fluorescence intensity compared to the reference solution, while other materials completely quenched QD fluorescence [8].

\subsubsection{Luminophores}

The ideal luminophore is environmentally stable and has a unity quantum yield (QY, the fraction of absorbed to emitted photons), so that all absorbed photons are re-emitted. 
It absorbs fully across the UV and visible spectrum before dropping to zero absorption immediately before its emission band.

In general, there are three classes of luminophores in use for LSCs, including dyes, QDs, and rare earth materials. These materials may be used individually or in combination with other materials from inside or outside of their class, such as dyes with other dyes or dyes with rare earth materials [9]. The absorption spectra of some example materials are shown in figure 1.2 .

Dyes were the original luminophore of choice and hold the current record for power conversion efficiency (PCE, defined as optical to electrical power) at 7.1\% (see section 1.2) [10]. However, dyes absorb over a relatively narrow spectral range and so are limited in their ability to absorb sunlight.

Much of the current LSC research involves QDs, and the majority of this research deals with Cd-salt QDs [11]. This is primarily due to the commercial availability of relatively high QY and stable Cd-salt QDs. Core/shell type QDs are typical, and advanced synthesis methods have led to investigations of many techniques to reduce self absorption losses such as type-II hetero-nanocrystals [12], doped QDs [13], and nanorods [14]. Using CdSe/CdS core/shell QDs, Coropceanu and Bawendi recently achieved optical efficiency $\eta_{\text {opt }}=48 \%[15]$ for an LSC device. Optical efficiency is a measure of how many photons are collected from the device per number of photons incident. But, this efficiency was only achieved for a very narrow range of spectral input. This is because Cd-salt QDs also have a relatively narrow absorption spectra, an example of which is shown in figure 1.2.

Historically, Pb-salt QDs with high enough QY to make them attractive for LSC work could not be fabricated. This has changed recently with the work of Evans et al., achieving up to $90 \%$ QY [17]. Pb-salt QDs have an attractive optical absorption spectrum, able to absorb across a region similar to silicon PV cells, as seen in figure 1.2.

Rare earth materials are perhaps the most seldom studied for LSC use [18]. This is because their absorption spectrum tends to be very narrow and QY can be quite low, especially when 


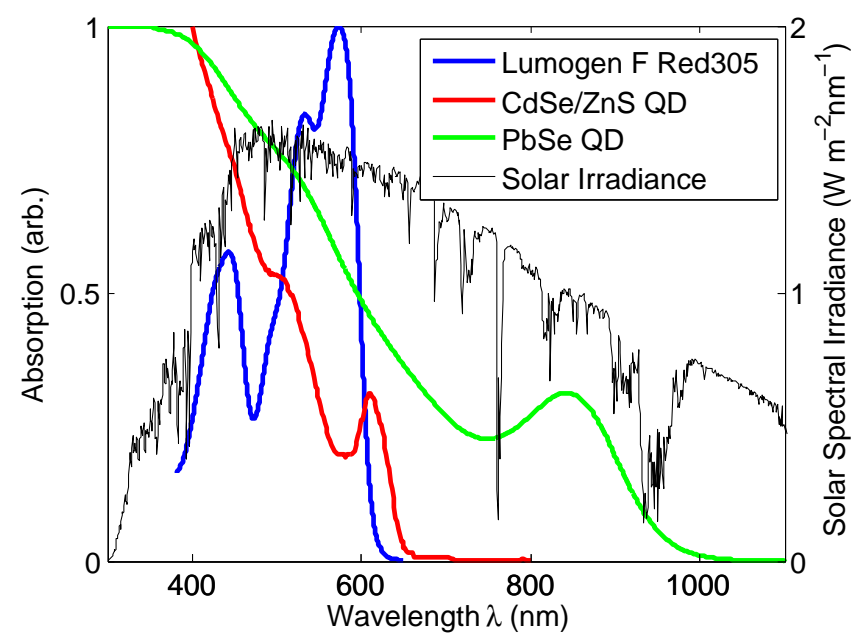

Figure 1.2: Comparison of the absorption spectra of Lumogen F Red 305 luminescent dye (measured), Cd-salt QD [16], and Pb-salt QD (measured) with the solar spectral irradiance at Earth's surface [6]. Pb-salt QDs are able to absorb across a considerably larger portion of the solar spectrum than dyes or Cd-salt QDs.

doped to increase their absorption [19]. They are interesting in that their absorption and emission spectra do not overlap, so they cannot "self absorb" photons emitted by another luminophore of the same type [20].

\subsubsection{Different LSC Architectures and Advancements}

There are many architectures beyond the standard single planar waveguide shown in figure 1.1. The simplest variant is a plate stack where each plate is doped with a luminophore that absorbs in a different region of the spectrum with a different PV cell type with matched band gaps bonded to each plate [4]. More solar energy can be absorbed in this fashion, but a greater solar cell area is required, negating some of the benefit. A single LSC plate can also be doped with multiple dyes, though they must be chosen more carefully in terms of their absorption and emission spectra. Cascading LSCs together (figure 1.3a) can reduce this extra cell area, but this design suffers greater optical loss [21].

Another simple change is to slowly taper the LSC towards the output edge (figure 1.3b), reducing the solar cell area and enhancing optical concentration by up to $30 \%$ [22]. Cylindrical designs can also be used to increase the optical concentration [23, 24], though mating to a 
(a)

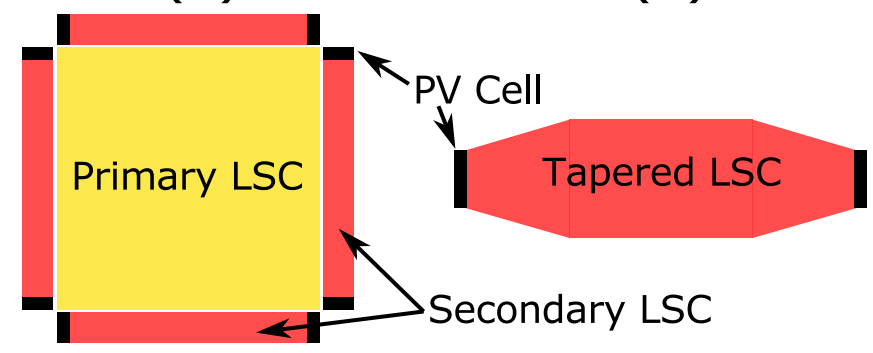

(c)

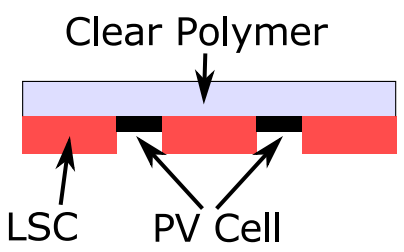

Figure 1.3: Various alternative LSC architectures: (a) Cascading primary and secondary LSCs; top view (b) Tapered LSC; side view (c) Front-facing LSCs; side view

PV cell may be difficult because of their circular shape. Dye-doped optical fiber mats have achieved 5.7\% PCE [25].

A variety of spectrally selective coatings can be applied to the absorbing surface of the LSC to reduce escape cone losses. It was demonstrated that using a cholesteric coating to reflect only the fluorescent light wavelengths, light output to the LSC edge can be increased by $12 \%$ [26]. An LSC design with a front-facing solar cell (figure 1.3c) was shown to reduce the cost per Watt of solar energy by $28 \%$ [27]. In this architecture, the PV cell faces the sun and collects sunlight directly, as well as photons emitted by the LSC which are guided to the PV cell face via TIR through the clear polymer top plate.

\subsection{State of the Art}

The current record for highest PCE of an LSC and PV cell system is 7.1\% [10]. This is held by a dye-based LSC featuring BASF Lumogen F Red 305 (absorption spectrum shown in figure 1.2) and Radiant Color Fluorescence Yellow CRS040 in a Plexit 55 PMMA (poly(methyl methacrylate)) resin matrix. Four GaAs solar cells are attached to the device, one per edge, connected electrically in parallel, for a geometric gain $G=2.5$ (defined as the ratio of collecting surface area to output edge surface area). A diffuse backside reflector was used with air gap.

The highest reported PCE for an LSC using a monocrystalline Si cell is $4.2 \%$ [28]. The 
device consisted of a two plate stack, one plate containing Red 305 dye and the other perylene perinone IR-emitting dye with $G=2.5$. Two edges were bonded to the Si cells, and two to mirrors; the assembly was placed over a diffuse backside reflector as before.

PCEs for QD-based LSCs are currently not as high and frequently unreported. The current best value is a PCE $=2.8 \%$ with $G=2.44$ using CdSe core/multi-shell QDs in poly(lauryl methacrylate-co-ethylene glycol dimethacrylate) attached to GaAs cells [29]. A PCE of $3.2 \%$ calculated from the LSC optical efficiency for a theoretical silicon PV cell has also been reported using $\mathrm{PbS}$ QDs [30]. However, this result is much less impressive, with $G=1.6$. In general, it is possible to achieve a higher PCE by lowering $G$.

\subsection{Other Applications of QD Nanocomposites}

Nanocomposites of QDs and polymers can be used as an optical sensor. Perhaps the most common is a temperature sensor which relies on a shift in fluorescence peak, change in fluorescent intensity, or other properties. One such device comprised of CdTe QDs in a PMMA waveguide had a $0.25 \frac{\mathrm{nm}}{{ }^{\circ} \mathrm{C}}$ shift in fluorescence peak and a quadratic drop in intensity [31]. There are many interesting specialty applications of this technology where a temperature probe cannot make good contact with an object to measure temperature without disturbing the object. Examples include nuclear magnetic resonance experiments [32], models in wind tunnels [33], and even individual animal cells [34]. Single-QD temperature sensors have also been demonstrated, allowing extremely high spatial resolution measurements [35].

QD-based sensors can also be used for chemical detection, with the target chemical altering the emission properties of the QD. One group constructed a nanocomposite to react to the presence of solvents like xylene for environmental remediation [36]. An $\mathrm{NO}_{2}$ gas sensor has also been demonstrated [37]. Radiation sensors for x-ray, neutron, gamma, and charged particle detection have also been investigated [38-40]. QD-based radiation sensors promise better room-temperature energy resolution and low afterglow due to their short 
photoluminescent decay time. And, they are not limited in size by crystal growth techniques like many current scintillators. Pb-salt QD nanocomposite sensors, especially, offer high x-ray attenuation and could improve sensitivity.

Steps towards biomedical imaging [41] and LED output spectrum adjustment [42] have also been demonstrated. Pb-salt QDs are special in that either their absorption or emission spectra can be made to overlap with fiber optic amplifier wavelength windows, allowing their use with a plethora of existing devices and technologies.

\subsection{Problem Statement}

It is estimated that carbon emissions by year 2050 could reach $50 \mathrm{Gt}$ (giga tonnes) per year, far above the $10 \mathrm{Gt}$ to $20 \mathrm{Gt}$ estimated as needed to keep global temperature rise below $2^{\circ} \mathrm{C}$ [43]. Solar energy will play an important and ever-increasing role in the energy generation mix. LSCs can reduce the cost of solar energy, adding an economic impetus for increasing solar energy generation.

LSCs have traditionally been limited by their inability to effectively absorb the solar spectrum while keeping optical losses at a manageable level. Dyes, while good at limiting losses with their high QY, absorb a very limited portion of the solar spectrum. Cd-salt QDs have not matched dyes because of a lower QY and only slightly improved absorption spectrum. Pb-salt QDs, on the other hand, can be tuned to absorb across the entire visible and near-IR spectrum. Pb-salt QDs are now poised to be a viable alternative to other luminophores as new chemical synthesis techniques have improved their QY [11]. This work focuses on investigations related to the use of PbSe QDs for LSCs to enhance overall photo-conversion efficiency. 


\section{Chapter 2: Fundamentals}

\subsection{Photovoltaic Cells}

While there are many types of solar cells, semiconductor-type photovoltaic (PV) cells [44-46] will be considered here, as they are most typically used with LSCs. When a photon is absorbed by a semiconductor material, an electron is promoted from the valence band to the conduction band leaving behind a vacancy in the valence band known as a hole. This electron-hole pair, known as an exciton, will move with a random diffusion current before quickly recombining in an undoped semiconductor.

However, by selectively doping the semiconductor as shown in figure 2.1, electricity can be generated due to the photovoltaic effect. One layer is doped with atoms which have more valence electrons than the host material, creating an electron-rich n-type material. A second layer is doped with atoms which have less valence electrons, creating an electron depleted hole-rich p-type material. Since these regions are in contact with one another, the excess electrons from the n-type material diffuse into the electron depleted p-type material. As the electrons diffuse, an electric field is set up in the "depletion" (or "space charge") region opposing the further diffusion of electrons because of the potential gradient $\Delta V$. This continues until an equilibrium is reached.

Now when a photon generates an exciton, the hole and electron are pulled in opposite directions in a phenomenon known as drift current in addition to the random diffusive movement. This drift current is driven by the electric field in an attempt to return to equilibrium. If the PV cell is connected to a load, electrons are allowed to flow from the n-type material, through the load, to the p-type material. In order for a photon to generate an exciton, it must have an energy greater than or equal to the band gap energy $\left(E_{\mathrm{g}}\right)$ of the material. 


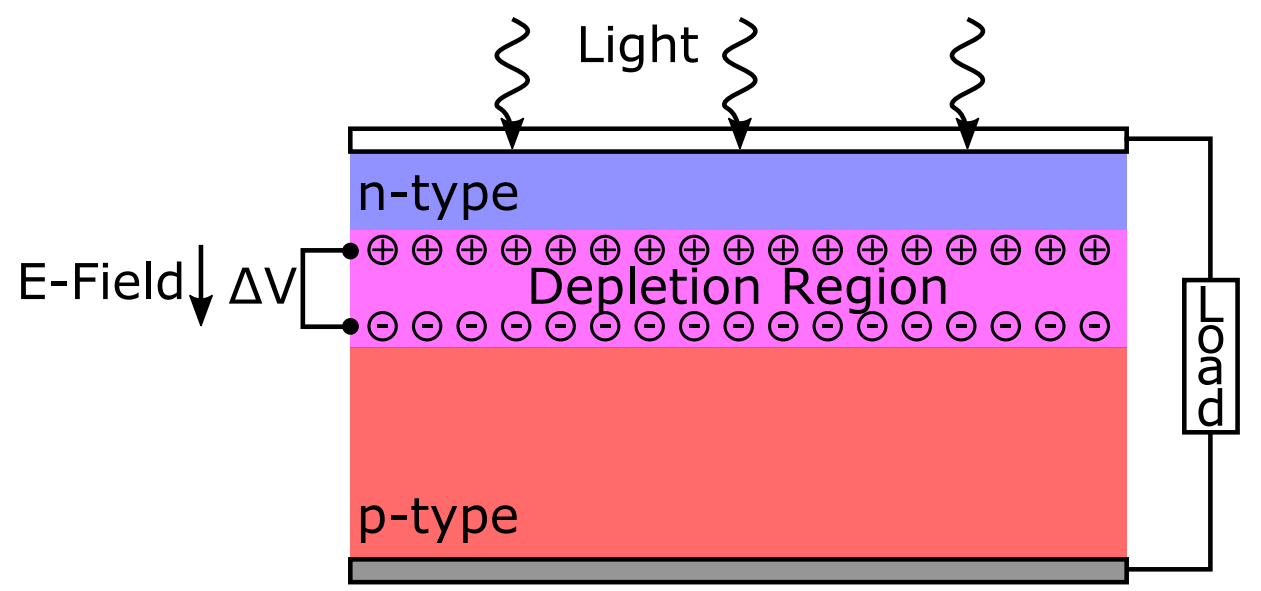

Figure 2.1: An example PV cell. Semiconducting material is n- and p-doped to form a junction across which electron and hole pairs can be separated to generate electricity. A load is attached to the top (clear) and bottom contacts to allow electrons to flow from the n- to p-type material.

The two most commonly used materials for PV cells with LSCs are monocrystalline silicon (Si) and gallium arsenide (GaAs). GaAs cells are preferred when their use is possible, owing to their higher efficiency. According to the National Renewable Energy Laboratory (NREL), the current verified world record efficiency for a single junction GaAs research grade cell is $(28.8 \pm 0.9) \%$ compared to $(25.6 \pm 0.5) \%$ for Si $[47]$.

The determining factor for which cell type to use is based on the emitting wavelength of the luminophore in the LSC. For GaAs, $E_{\mathrm{g}}=1.42 \mathrm{eV}$, compared to $E_{\mathrm{g}}=1.12 \mathrm{eV}$ for Si [48]. Thus excitons can be generated in GaAs by photons with $\lambda \leq 870 \mathrm{~nm}$, and in Si by photons with $\lambda \leq 1100 \mathrm{~nm}$.

\subsection{Quantum Dots}

Quantum dots (QDs) were first studied by Ekimov and Onuschchenko in the former USSR in glass matrices in 1981 [49,50], and shortly after in the United States by Brus [51,52]. They are nanocrystals of semiconducting material, usually $2-10 \mathrm{~nm}$ in diameter, and are sometimes termed "artificial atoms" because they demonstrate properties between those of bulk materials and individual molecules. They often have between a few hundred and a few 
thousand atoms each. The properties of bulk materials begin to shift towards properties of molecules when they have a radius of less than the exciton Bohr radius (section 2.2.2) and thus confine their excitons in all three spatial dimensions (or fewer dimensions in the case of films or tubes).

They are most known for their band gap energy being a function of crystal size. This can be seen in figure 2.2 as a change in their fluorescence wavelength. QDs are frequently specified in terms of their material type, fluorescence wavelength, lowest energy absorption peak wavelength, or quantum yield (QY). The QY represents the ratio of fluorescence photons to photons absorbed by a QD.

\subsubsection{Physical Structure}

QDs come in many shapes, including pyramidal [53], disk [54], rod, arrow, teardrop, tetrapod [55], and others, though spherical is most common. Most have a physical structure like those shown in figure 2.3 with a semiconductor core surrounded sometimes with a shell of another semiconducting material, and usually coated with some sort of ligand for protection and/or functionalization.

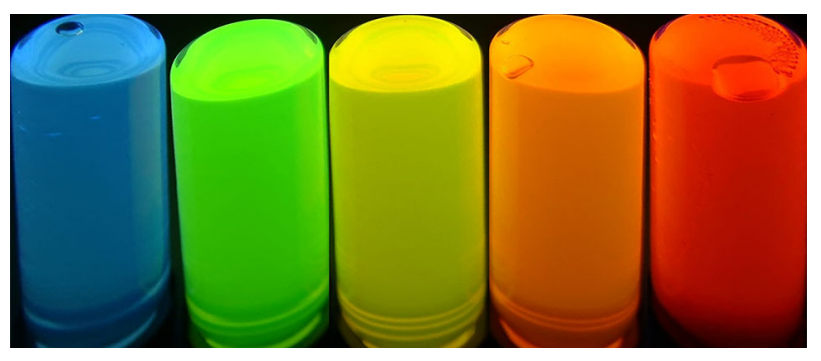

Figure 2.2: Different sized CdSe QDs displaying various colors of fluorescence through a change in their band gap energy. The smallest QDs fluoresce blue while larger QDs fluoresce at longer wavelengths. (Modified from photo by M.S. Wong [56]).

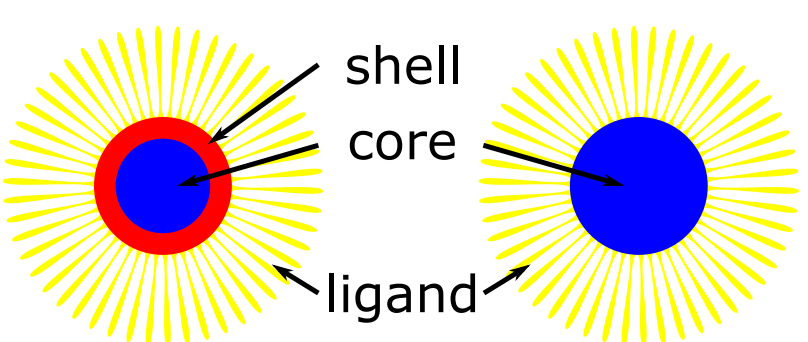

Figure 2.3: Physical structure of the common spherical quantum dot. A core of semiconducting material can be coated with a protective shell of a different semiconducting material for a core/shell configuration or left unshelled. QDs are usually coated with an additional protective and/or functionalizing ligand layer. 
The core material and size is the most important factor in setting a QD's optical properties. The shell material is primarily to passivate the QD surface and increase the QY by removing surface trap states while protecting the QD from environmental degradation and oxidation $[57,58]$. However, a carefully synthesized QD shell can be used to further optimize QD optical properties. The shell can be engineered to increase the Stokes shift [59,60], tune the aggregate band gap to a region not accessible to either semiconductor individually $[58,61]$, segregate the electron and hole of an exciton to different physical locations within the QD [62], or alter other properties.

The ligand layer helps to protect the QD, but it can also be tailored to determine what happens to the charge carriers once a photon is absorbed by the QD. Electrically insulating ligands will maximize fluorescence and decrease conductivity and inter-particle charge transfer, while shorter or conductive ligands suppress luminescence and increase charge transfer [63-65]. Ligands can also functionalize the QD. For example, they can help to stabilize the fluorescence in polymers [66], affect their aggregate morphology [67], create conditions for self-assembly of QD structures [68], and make the QD water soluble for use in vivo [69].

\subsubsection{Exciton Bohr Radius}

The exciton Bohr radius $r_{\mathrm{B}}$ is the crystal radius at which it will begin to take on properties that differ from the bulk material. It can be derived in the same way as the standard Bohr radius of a hydrogen atom $[70,71]$. When a crystal is smaller than $r_{\mathrm{B}}$, electrons will be physically confined to a space less than the natural size of their orbit, giving rise to a change in physical properties.

The exciton Bohr radius is given by:

$$
r_{\mathrm{B}}=\frac{4 \pi \hbar^{2} \epsilon}{e^{2}}\left(\frac{1}{m_{\mathrm{e}}^{*}}+\frac{1}{m_{\mathrm{h}}^{*}}\right)
$$

where $\hbar$ is the reduced Planck constant, $\epsilon=\epsilon_{\mathrm{r}} \epsilon_{0}$ is the dielectric constant of the material, 
and $m_{\mathrm{e}}^{*}$ and $m_{\mathrm{h}}^{*}$ are the effective masses of electrons and holes. The commonly accepted value of $r_{\mathrm{B}}$ for $\mathrm{PbSe}$ is $46 \mathrm{~nm}$, one of the largest known Bohr radii of any semiconductor [72].

\subsubsection{Electronic and Optical Spectra}

When a semiconductor crystal lattice is smaller than $r_{\mathrm{B}}$, changes in its optical spectra result from shifts in its electronic band structure, enhancing optical properties such as absorption coefficient and fluorescence intensity [70,73-76].

As the number of atoms drops, the density of states can no longer be considered infinite. The valence and conduction bands separate into discrete states and the band gap energy $E_{\mathrm{g}}$ increases, as shown in figure 2.4. This continues until the case of a single molecule, at which point only the highest occupied molecular orbital (HOMO) and lowest unoccupied molecular orbital (LUMO) are considered. For simplicity, only the lowest energy band, the 1-s band, is shown.

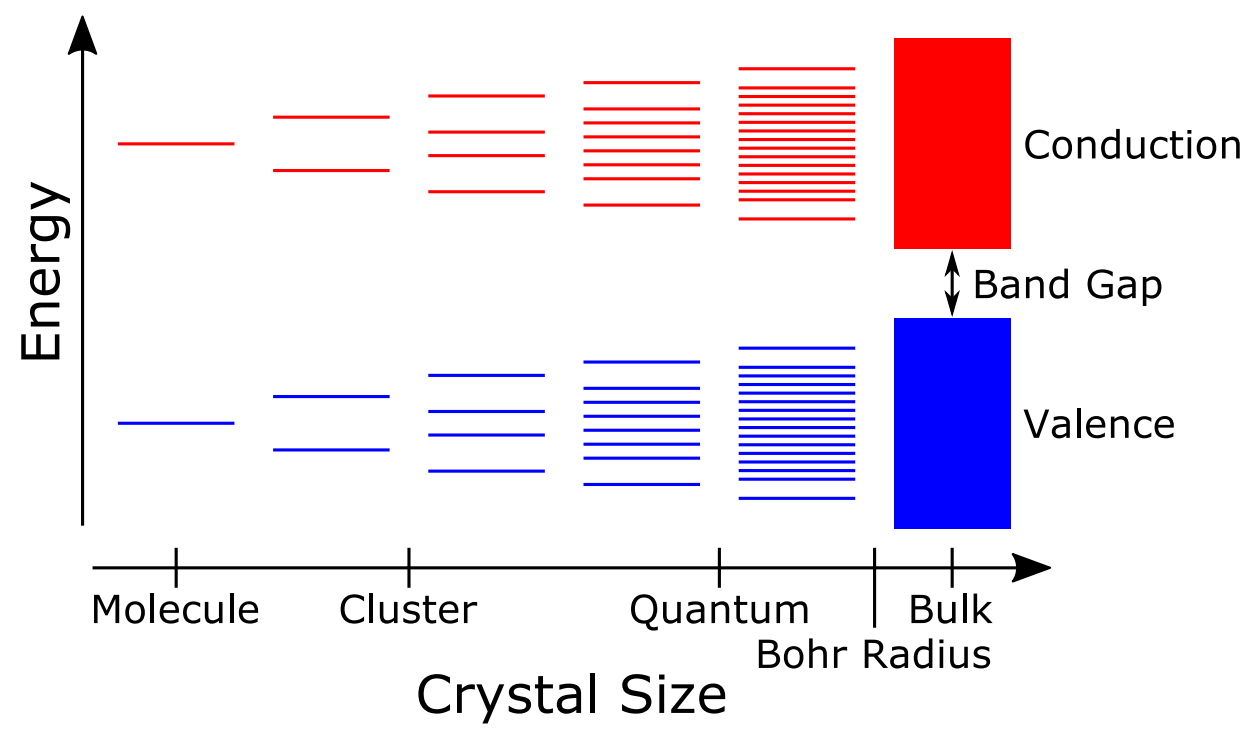

Figure 2.4: Diagram of the valence and conduction bands in an arbitrary material (only 1-s bands shown). The density of states increases with the number of atoms until the energy levels become continuous in the bulk material with a radius larger than $r_{\mathrm{B}}$. Adapted from A.J. Houtepen [77]. 


\section{Absorption}

Optical absorption occurs for any energy where there is an allowed electronic transition. A simple illustrative model is shown in figure 2.5. Here, there are two states in both the valence and conduction bands. Assuming all of these transitions are allowed, there would be an absorption line at each energy $E_{1}$ through $E_{4}$. In this simplistic model, these lines appear as delta functions, shown as the solid blue lines on the right of figure 2.5, because absorption can only happen at exactly these energies. It is important to note that not all electronic transitions are formally allowed within a system as they must follow the Pauli Exclusion Principle and the Laporte Selection Rule.
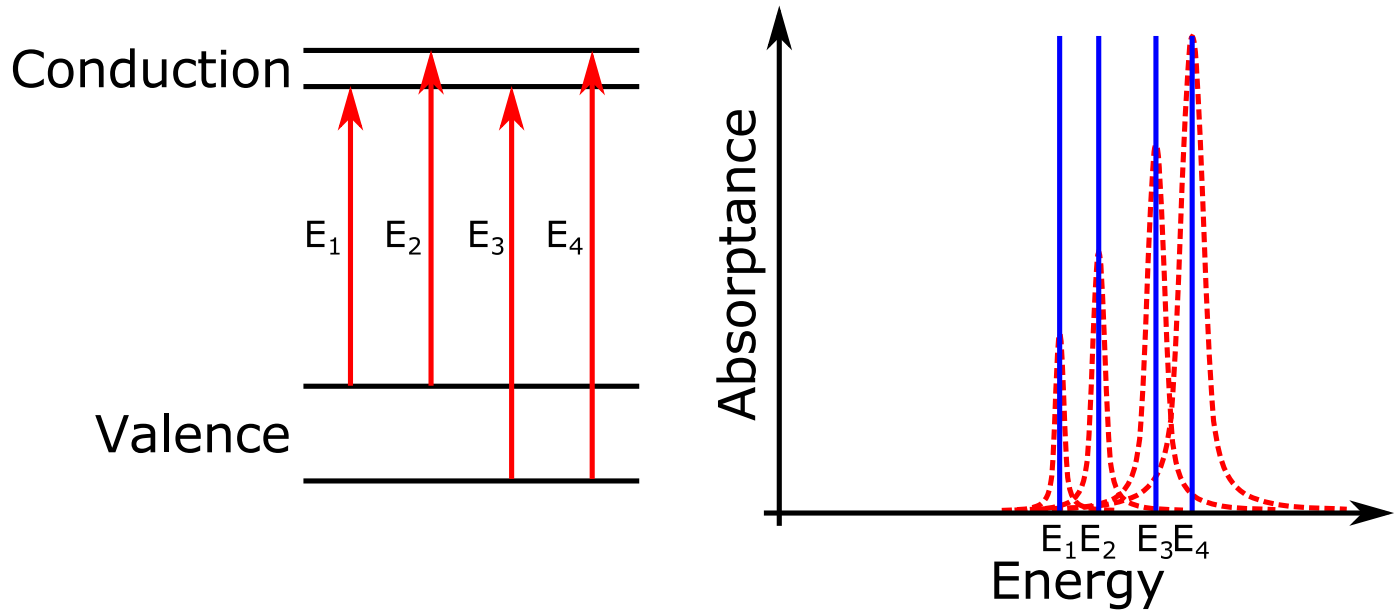

Figure 2.5: A simple model of semiconductor absorption spectra. Left: Photons with energy $E_{1-4}$ raise electrons from the valence to conduction band. Right: These electronic transitions correspond to absorption lines at the energy of the transition. Theoretically, these lines are delta functions, but homogeneous broadening results in a Lorentzian curve centered on the delta function. The area under each Lorentzian curve corresponds to the probability of that electronic transition and therefore the optical absorption at that energy.

In reality, there is a minimum frequency bandwidth over which the absorption will occur; this is referred to as homogeneous broadening. This broadening of the delta function can be represented as a Lorentzian function, shown as the red dotted lines on the right of figure 2.5. The area under the Lorentzian curve is proportional to the probability of that electronic transition and therefore the probability of absorption at a particular photon energy. 
The absorption spectra of a sample of QDs tends not to show these perfect Lorentzian peaks. There are numerous sources of broadening which will make the absorption appear as a continuous curve, usually only with a broad 1-s peak obvious in the case of QDs. One source of this inhomogeneous broadening is the splitting of each valence band into multiple bands caused by the effective mass assumption breaking down for the valence band. Each electronic state can also have numerous vibrational energy levels, one or more of which may be allowed transitions. The superposition of these nearby Lorentzian peaks appears Gaussian, and many of these Gaussian sets of peaks can make up the entire absorption curve.

Additional inhomogeneous band broadening is a result of lattice vibrations and coupling to phonons. This is a result of the lattice not being perfectly rigid as assumed in the simple model above. Phonon coupling is stronger at higher temperatures, resulting in greater broadening at higher temperatures, as discussed in section 2.2.5.

\section{Emission}

Once a QD absorbs a photon and an electron is promoted to the excited state, the excited electron will almost always undergo complete relaxation to the band edge via vibrational or intraband relaxation (internal conversion), a principle known as Kasha's rule [78]. Within the excited state these transitions are very fast, but the transition from the excited to ground state via these mechanisms is much slower, allowing other processes such as fluorescence to be competitive.

Therefore, the emission spectral shape depends on the nature of the optical transitions [79]. If all of the transitions in a luminophore are to various vibrational states of the same electronic state, the emission spectrum will show mirror symmetry with the absorption spectra, as shown on the left of figure 2.6. Upon relaxing to the band edge, the lowest vibrational state, the electron can relax to any of the allowed vibrational states in the ground electronic state, resulting in multiple emission peaks mirroring the absorption spectrum peaks. If instead the transitions occur only between different electronic levels, the electron will relax to the band 
edge and only have a single ground state to which it can relax. A single emission peak is seen in this case, as shown on the right of figure 2.6. Many dyes show at least partial symmetry of absorption and emission spectral shape, while QDs tend to have a single emission peak.

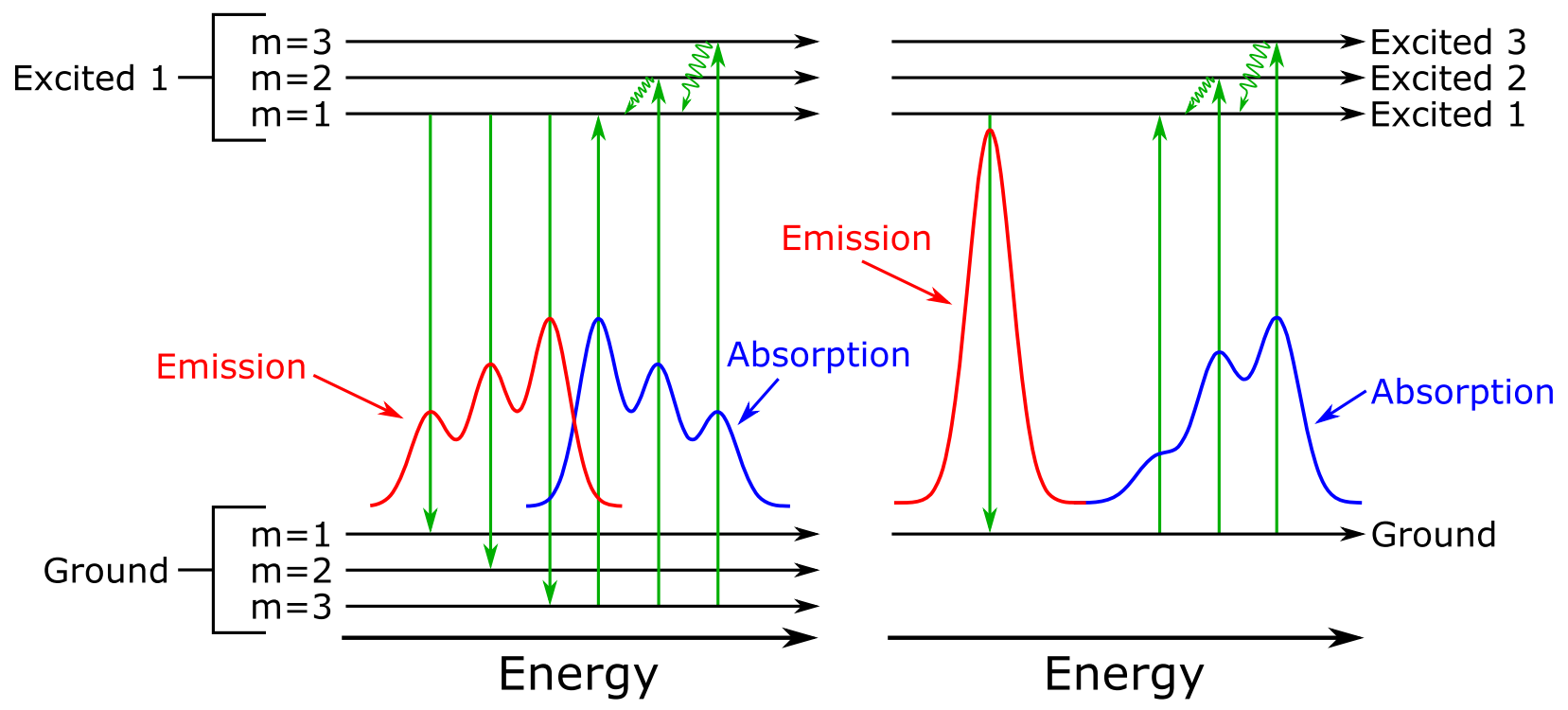

Figure 2.6: Relationship of the emission spectra to the absorption spectra. Left: Example of a mirrored spectra shape involving only one electronic excited state and three vibrational ( $m=1,2,3)$ states. Right: Example of a non-mirrored spectra shape involving three electronic excited states and no vibrational states. Real luminophores may have a combination of these types of transitions. Sinuous lines are vibrational and intraband relaxations to the band edge. Adapted from Herman et al. [79].

Many of the same processes which apply to the broadening of the absorption spectrum also apply to emission.

\section{Stokes Shift}

The Stokes shift is the difference in energy between the lowest energy absorption peak and the highest energy emission peak. This shift is a result of lattice vibrations and can be demonstrated with the configurational coordinate diagram in figure 2.7. $Q$ is the configurational or nuclear coordinate referenced to the optically active center. Here, a simple two electronic state system, ground and excited, is used for demonstration.

In this system, ground and excited states take on a parabolic shape comprised of many vibrational states due to harmonic lattice vibrations. The lowest energy equilibrium ground 


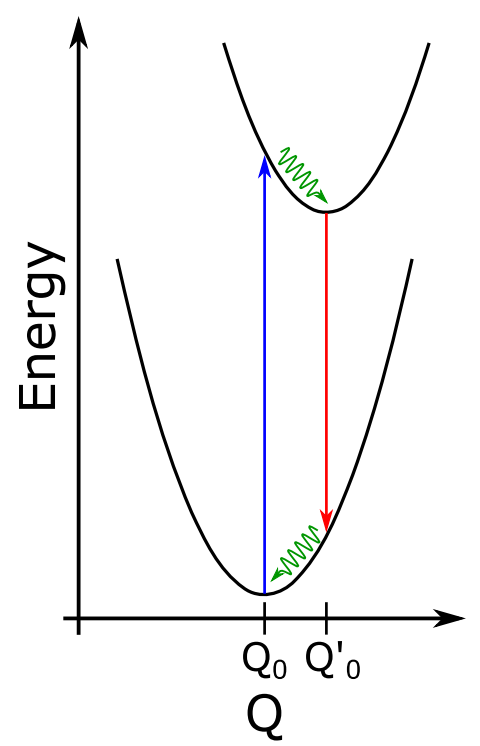

Figure 2.7: Configurational coordinate diagram showing the origin of the Stokes shift. A photon excites an electron residing at the ground state equilibrium position $Q_{0}$ to the excited state. The electron vibrationally relaxes to the excited state equilibrium $Q_{0}^{\prime}$ before radiatively relaxing back to the ground state. Finally, the electron vibrationally relaxes a second time back to the ground state equilibrium position $Q_{0}$. The two vibrational relaxations (sinuous lines) make up the Stokes shift. A larger $\Delta Q$ results in a larger Stokes shift. Adapted from Solé et al. [73].

state occurs at $Q_{0}$. The lowest energy equilibrium excited state is held some distance $\Delta Q=Q_{0}^{\prime}-Q_{0}$ away at a position $Q_{0}^{\prime}$. When an electron is promoted to the excited electronic state, it initially does not change in $Q$, so it is now in a non-equilibrium vibrational state. It quickly vibrationally relaxes to the excited equilibrium state at $Q_{0}^{\prime}$, shown as the sinuous line. The electron relaxes to the ground state, again initially not changing in $Q$. Finally it vibrationally relaxes back to equilibrium at $Q_{0}$.

The origin of the Stokes shift can now be understood as the energy lost in the two vibrational relaxation steps which must always occur. Certain systems have a higher $\Delta Q$ and so have a greater intrinsic Stokes shift. Other extrinsic sources of Stokes shift such as trap states are also possible. 


\section{Förster Resonance Energy Transfer}

Förster Resonance Energy Transfer, or FRET, is the non-radiative transfer of energy from one luminophore to another through dipole-dipole coupling, as shown in figure 2.8 [80-83]. The probability of transfer depends on their separation in space, mutual orientation of dipoles, and spectral overlap of the donor's emission spectrum with the acceptor's absorption spectrum.

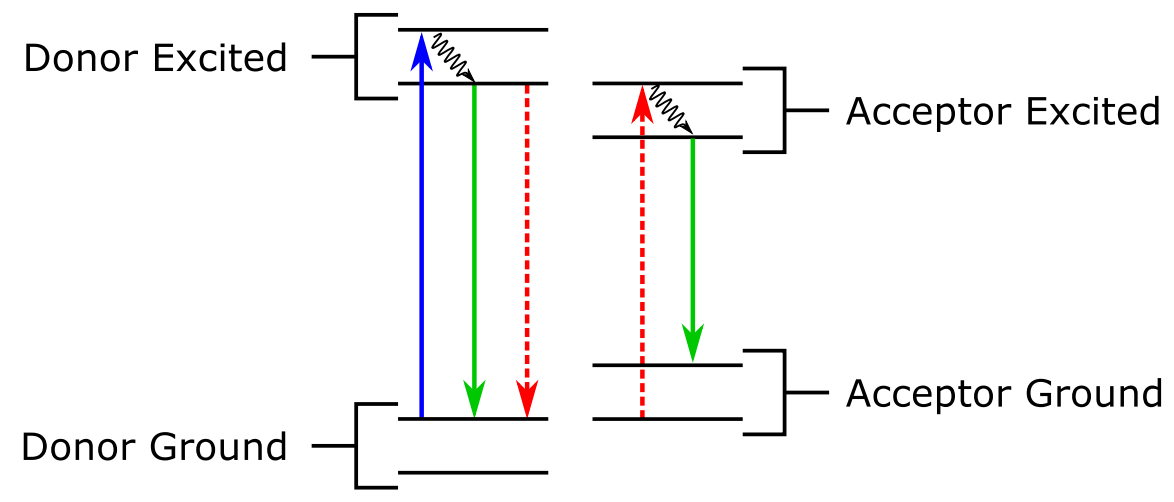

Figure 2.8: Diagram of FRET. The blue arrow is absorption, green are emission (radiative decay), and dotted red are FRET. Sinuous lines are non-radiative decay. If a donor-acceptor pair are close enough together, an excited donor can either emit a photon and relax, or transfer its energy via FRET to the acceptor.

For an isotropic system, one in which the luminophores have no correlation of their dipole orientation, their orientation can be ignored. The FRET transfer efficiency between a donor-acceptor pair is then:

$$
\Phi_{\mathrm{T}}=\frac{1}{1+\left(r / r_{0}\right)^{6}}
$$

where $r$ is the donor-acceptor separation and $r_{0}$ is the Förster distance. Förster distance is defined as the separation at which the rates of FRET and radiative emission are equal, usually a few nm. $r_{0}$ is a function of the spectral overlap of the donor-acceptor pair.

Because FRET will cause a red shift in the observed fluorescence of a sample, it provides a simple way of monitoring luminophore dispersion in a matrix. If the luminophores are not well dispersed and have formed clumps, the observed fluorescence will red shift and loose intensity. Especially in the case of QDs where aggregation also means reduced confinement, this should be avoided. 


\subsubsection{Brus Model}

In 1984, Brus established a model for predicting the change in band gap energy $\Delta E_{\mathrm{g}}$ from bulk material based on QD radius and bulk material properties [51,52,84]:

$$
\Delta E_{\mathrm{g}}=\frac{h^{2}}{8 \mathrm{R}^{2}}\left(\frac{1}{m_{\mathrm{e}}^{*}}+\frac{1}{m_{\mathrm{h}}^{*}}\right)-\frac{1.8 e^{2}}{4 \pi \epsilon_{\mathrm{QD}} \epsilon_{0} \mathrm{R}}+\frac{e^{2}}{\mathrm{R}} \sum_{\mathrm{k}=1}^{\infty} \alpha_{\mathrm{k}}\left(\frac{S_{\mathrm{e}, \mathrm{h}}}{\mathrm{R}}\right)^{2 \mathrm{k}}
$$

where $h$ is Planck's constant, $\mathrm{R}$ is the $\mathrm{QD}$ radius, $m_{\mathrm{e}}^{*}$ and $m_{\mathrm{h}}^{*}$ are the effective masses of electrons and holes, $e$ is the elementary charge, $\epsilon_{\mathrm{QD}}$ is the dielectric constant of the $\mathrm{QD}, \epsilon_{0}$ is vacuum permittivity, $S_{\mathrm{e}, \mathrm{h}}$ represents the position of an electron and hole, and $\alpha_{\mathrm{k}}$ is a term in the polarization energy which deals with the dielectric constants of the QD and matrix materials. Since the electron and hole are confined to the same space, their positions may be considered with a single term. The bar in the third term signifies an average over the wave function $\Psi_{1}\left(\bar{S}_{\mathrm{e}, \mathrm{h}}\right)$.

The model makes the assumption that the QD is spherical, the interior of the QD is uniform, and the potential energy outside of the QD is infinite. The three terms of this model, kinetic energy, Coulomb energy, and polarization energy, are discussed in more detail below. Coulomb energy and polarization energy together represent potential energy.

\section{Kinetic Energy}

The first term in the model, kinetic energy $E_{\mathrm{k}}$, dominates the prediction, as shown in figure 2.9. Frequently, $E_{\mathrm{k}}$ will be used alone as the predictor of $\Delta E_{\mathrm{g}}$ for this reason. This term follows directly from the solution for the Schrödinger equation for a particle in a box, modified for a sphere. This solution has the wave function:

$$
\Psi_{l}(r)=\frac{1}{r \sqrt{2 \pi \mathrm{R}}} \sin \left(\frac{l \pi r}{\mathrm{R}}\right)
$$


and energy levels:

$$
E_{l}=\frac{h^{2} l^{2}}{8 \mathrm{R}^{2} m_{\mathrm{c}}^{*}} \quad \text { for } l=1,2,3 \ldots
$$

where $m_{\mathrm{c}}^{*}$ is the effective mass of the charge, $r$ is the distance of the charge from the center of $\mathrm{QD}$, and $\mathrm{R}$ is the $\mathrm{QD}$ radius. $E_{\mathrm{k}}$ then follows directly with $l=1$ and

$$
\frac{1}{m_{\mathrm{c}}^{*}}=\frac{1}{m_{\mathrm{e}}^{*}}+\frac{1}{m_{\mathrm{h}}^{*}} .
$$

The measured result shown in figure 2.9 differs significantly from the Brus prediction. This is a known limitation of the Brus model wherein the effective mass approximation used to calculate $E_{\mathrm{k}}$ breaks down for small R. For $E_{\mathrm{k}}>\sim 0.5 \mathrm{eV}$, further increase in $E_{\mathrm{k}}$ is substantially less rapid than the Brus model predicts. A more complex model of effective mass would be required to address this issue.

For QDs with small R, low effective mass, and small bulk $E_{\mathrm{g}}$, the surface carrier charge density is no longer negligible. If the surface charge density is important, this simplified model will again overestimate the value of $E_{\mathrm{k}}$.

The anisotropy factor of $m_{\mathrm{e}}^{*}$ and $m_{\mathrm{h}}^{*}$ is up to $2-3 \times$ in PbSe [85] and also needs to be considered. An overly simplistic way to account for this anisotropy is to use larger effective mass values, such as $m_{\mathrm{e}}^{*} \approx m_{\mathrm{h}}^{*} \approx 0.2 m_{0}$. The predicted value at $\mathrm{R}=1.25 \mathrm{~nm}$ becomes $\lambda_{\mathrm{g}}=471.2 \mathrm{~nm}$ instead of $\lambda_{\mathrm{g}}=246.3 \mathrm{~nm}$ as shown in figure 2.9. The measured value is $\sim 850 \mathrm{~nm}$.

\section{Coulomb Energy}

The Coulomb energy $E_{\mathrm{C}}$ component is the potential energy due to force between the electron and hole point charges, given by Coulomb's law:

$$
E_{\mathrm{C}}=-\int_{r=0}^{\mathcal{R}} k_{\mathrm{e}} \frac{\left|q_{1} q_{2}\right|}{\epsilon_{\mathrm{r}} r^{2}} d r \quad k_{\mathrm{e}}=\frac{1}{4 \pi \epsilon_{0}}
$$


where $q_{1}=e$ is the charge of an electron, $q_{2}=-e$ is the charge of a hole, $\epsilon_{\mathrm{r}}=\epsilon_{\mathrm{QD}}, k_{\mathrm{e}}$ is Coulomb's constant, and $\mathcal{R}=\frac{\mathrm{R}}{1.8}$, the average separation between the charges. $\mathcal{R}$ is found via perturbation theory and solving over the wave function of the system $[84,86]$. Then:

$$
E_{\mathrm{C}}=-\frac{1.8 e^{2}}{4 \pi \epsilon_{\mathrm{QD}} \epsilon_{0} \mathrm{R}}
$$

\section{Polarization Energy}

The polarization energy $E_{\text {pol }}$ depends on the dielectric constant of the external matrix. By inserting the value of $\alpha_{k}$ :

$$
\alpha_{k}=\frac{(\epsilon-1)(k+1)}{4 \pi \epsilon_{\mathrm{QD}} \epsilon_{0}(\epsilon k+k+1)} \quad \epsilon=\frac{\epsilon_{\mathrm{QD}}}{\epsilon_{\mathrm{mat}}}
$$

$E_{\text {pol }}$ can be solved numerically, averaged over the wave function $\Psi_{1}[84,87]$ :

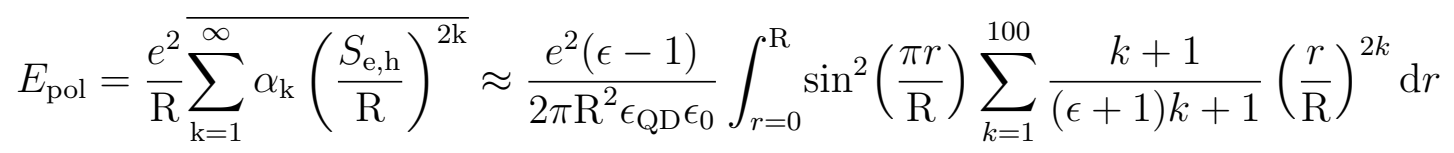

where $\epsilon=\epsilon_{\mathrm{QD}} / \epsilon_{\mathrm{mat}}, \epsilon_{\mathrm{mat}}$ is the matrix dielectric constant, and $\epsilon_{\mathrm{QD}}$ is the QD material bulk dielectric constant. A very good approximation of $E_{\text {pol }}$ can be made by numerically computing a truncated sum with $k=1 \rightarrow 100$ because $E_{\text {pol }}$ is small compared to $E_{\mathrm{k}}$ and $E_{\mathrm{C}}$ and the $(r / R)^{2 k}$ term rapidly goes to zero as $k$ increases.

\section{Brus Model Prediction}

The model prediction for PbSe QDs in hexane is plotted in figure 2.9. The following values were used: $m_{\mathrm{e}}^{*} \approx m_{\mathrm{h}}^{*} \approx 0.1 m_{0}$ where $m_{0}$ is the mass of an electron [85,88,89], $\epsilon_{\mathrm{QD}}=22.9[90]$, $\epsilon_{\mathrm{mat}}=1.89$ (hexane) [91], and bulk band gap $E_{\mathrm{g}, \mathrm{bulk}}=0.28 \mathrm{eV}$ [89]. For comparison, the band gap wavelength of measured PbSe QDs in hexane is shown. 


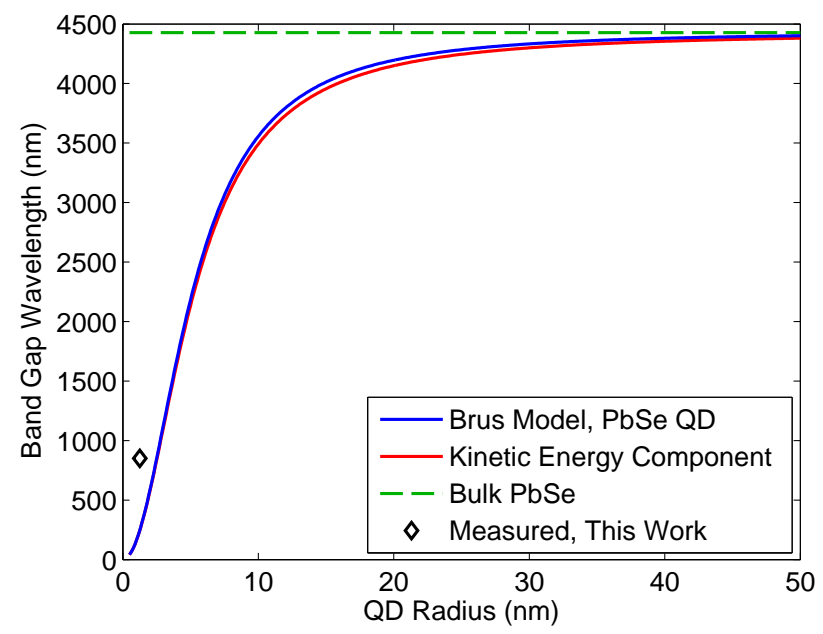

Figure 2.9: Brus model prediction for PbSe QDs in hexane. As expected, the QD band gap wavelength approaches bulk behavior as the QD gets larger, and moves to shorter wavelengths as the QD gets smaller. The kinetic energy term dominates, deviating only slightly from the prediction of the entire model. The band gap wavelength of measured PbSe QDs in hexane is shown for comparison with the model.

\subsubsection{Effect of Temperature}

\section{Band Gap Energy}

The shift in optical properties with temperature is a result of shifting electronic energies of the QD. This effect is not unique to QDs: an early model was put forth by Varshni in 1967 for bulk semiconductor band gap energy $E_{g}$ change with temperature $T$ in $\mathrm{K}[92]$ :

$$
E_{\mathrm{g}}(T)=E_{0}-\alpha \frac{T^{2}}{T+\beta}
$$

where $E_{0}$ is the band gap energy at $0 \mathrm{~K}$ and $\alpha$ and $\beta$ are material constants.

This model predicts the change in $E_{g}$ based on lattice dilation with temperature (distinct from expansion of the QD radius) and the shift in relative position of the conduction and valence bands from an intensifying electron-lattice interaction at higher temperatures. It accurately predicts the behavior of Cd-salt QDs [93], but it is only valid for group IV and III-V semiconductors and does not account for the effects of strong confinement in Pb-salt QDs [92, 94, 95]. 
Pb-salt QDs have large Bohr radii, $46 \mathrm{~nm}$ for excitons and $\sim 23 \mathrm{~nm}$ for both holes and electrons, making them special among QDs in that both holes and electrons - not just excitons - are strongly confined [72]. PbSe also has a very large change of carrier effective mass with temperature: electrons vary as $T^{0.35}$ and holes as $T^{0.45}$ in the range $100 \mathrm{~K}$ to $400 \mathrm{~K}[96]$. These factors combine to make confinement energy the dominant factor in affecting the band gap with temperature in Pb-salt QDs in the strong confinement (small radius) regime $[72,97,98]$.

Pb-salt QDs show a lower change in band gap energy with temperature as the QD radius is lowered, to the point of becoming null or negative if the QD is small enough (i.e. $\frac{d E_{g}}{d T}$ is greater at larger radii) [97]. The Stokes shift gets larger as the QD gets smaller and confinement energy increases [99-101].

\section{Full width at half-maximum}

The full width at half-maximum (FWHM) of QD fluorescence is predicted to increase with temperature, a result of the increased interaction with and scattering of excitons by longitudinal optical (LO) phonons. FWHM $(\Gamma$, in meV) as a function of temperature ( $T$ in $\mathrm{K})$ is given by a modified Bose-Einstein relation $[102,103]$ :

$$
\Gamma(T)=\Gamma_{\mathrm{inh}}+\gamma_{\mathrm{AC}} T+\frac{\Gamma_{\mathrm{LO}}}{\exp \left[\frac{E_{\mathrm{LO}}}{k_{\mathrm{B}} T}\right]-1}
$$

where $\Gamma_{\text {inh }}$ is the inhomogeneous broadening, $\gamma_{\mathrm{AC}}$ is the exciton-acoustic phonon scattering coefficient, $\Gamma_{\mathrm{LO}}$ is the exciton-LO phonon coupling strength, $E_{\mathrm{LO}}$ is the LO-phonon energy, and $k_{B}$ is the Boltzmann constant. $E_{\mathrm{LO}}$ is a material parameter which will range from about $20 \mathrm{meV}$ to $40 \mathrm{meV}$ depending on the size of the $\mathrm{QD}[104] . \Gamma_{\mathrm{inh}}, \Gamma_{\mathrm{LO}}$, and $\gamma_{\mathrm{AC}}$ can be fit from the temperature dependent FWHM data; data starting near $0 \mathrm{~K}$ would be required to properly fit these variables. Additionally, the accuracy of the prediction falls above $200 \mathrm{~K}[102,104]$. 
Another model which also predicts a linear broadening near room temperature is $[73,105]$ :

$$
\Gamma(T) \approx \Gamma(0) \sqrt{\operatorname{coth}\left(\frac{E_{\mathrm{LO}}}{2 k_{\mathrm{B}} T}\right)} .
$$

This model applies to both absorption and emission peak broadening.

\section{Fluorescence Intensity}

The fluorescence of QDs is quenched with increasing temperatures as the probability of non-radiative decay processes increases. This change can be reversible (the fluorescence properties recover when brought back to room temperature) or irreversible (the change in fluorescence properties is permanent). There are many potential mechanisms, including surface trap states, Auger-like phenomena, thermally activated crossover, and multiple phonon relaxation $[73,106-113]$.

Trap states can be a result of reversible thermally activated processes or by permanent structural changes such as ligand dislocation, oxidation, or lattice imperfections. Carrier escape to trap states is further enhanced by increasing longitudinal optical phonon scattering with increasing temperature. $[112,113]$

Auger-like phenomena occur through the electron-hole Coulomb interaction in semiconductors where conditions allow a hole to relax more quickly than an electron, such as when the effective mass of a hole is larger than that of an electron $[111,114,115]$. Auger-like electronhole energy transfer can then occur, causing carrier relaxation. This mechanism is unlikely in PbSe QDs, owing to the very similar effective masses of holes and electrons $[85,88,89]$.

Crossover is a process by which a carrier is allowed to relax to the ground state from the excited state with an activation energy $E_{\text {a }}$ provided by thermal phonons [73]. The decrease in luminescence intensity $I$ with increasing temperature for this process is proportional to [107-109]:

$$
\frac{I}{I_{0}} \propto \frac{1}{1+a\left(\exp \left[\frac{-E_{\mathrm{a}}}{k_{\mathrm{B}} T}\right]\right)}
$$


where $I_{0}$ is the fluorescence intensity at $T=0 \mathrm{~K}, k_{\mathrm{B}}$ is Boltzmann's constant, and $a$ is a fitting parameter representing the probability of the process occurring.

Finally, multiple phonon relaxation occurs when one or more phonons are emitted simultaneously to bridge the gap between excited and ground states [106]. The decrease in luminescence intensity $I$ with increasing temperature for this process is proportional to $[107-109]$ :

$$
\frac{I}{I_{0}} \propto \frac{1}{1+b\left(\exp \left[\frac{E_{\mathrm{LO}}}{k_{\mathrm{B}} T}\right]-1\right)^{-m}}
$$

where $E_{\mathrm{LO}}$ is the energy of longitudinal optical phonons, $m$ is the number of LO phonons involved in relaxation, and $b$ is a fitting parameter like $a$.

\subsection{Geometric Solar Concentrators}

\subsubsection{Overview}

Geometric solar concentrators operate by reflecting and/or refracting light to focus on to a PV cell. Initially, many designs were based on a single parabolic mirror in a configuration known as point focus [116], as shown in figure 2.10a. These designs reflect and focus light to a single point (three dimensional focusing) or to a line (two dimensional focusing). An evolution of this idea is the compound parabolic reflector (CPC) [117-119], shown in figure 2.10b, which is able to focus more diffuse rays. Instead of directly focusing the incoming rays, they are "funneled" to the absorbing surface.

Lenses are also an obvious choice for solar concentrators. Fresnel lenses are popular for this application because they are thinner, lighter, cheaper, and image quality is not an issue [120-122]. An example Fresnel lens is shown in figure 2.10c.

Concentrating via these methods can result in hot spots, areas of much higher intensity, which can be detrimental to performance and damaging to the PV cell. Some work is being done to mitigate these effects; for example, specially structured reflectors and secondary 


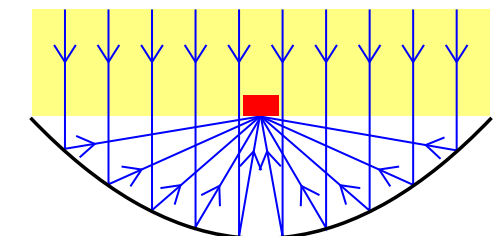

(a)
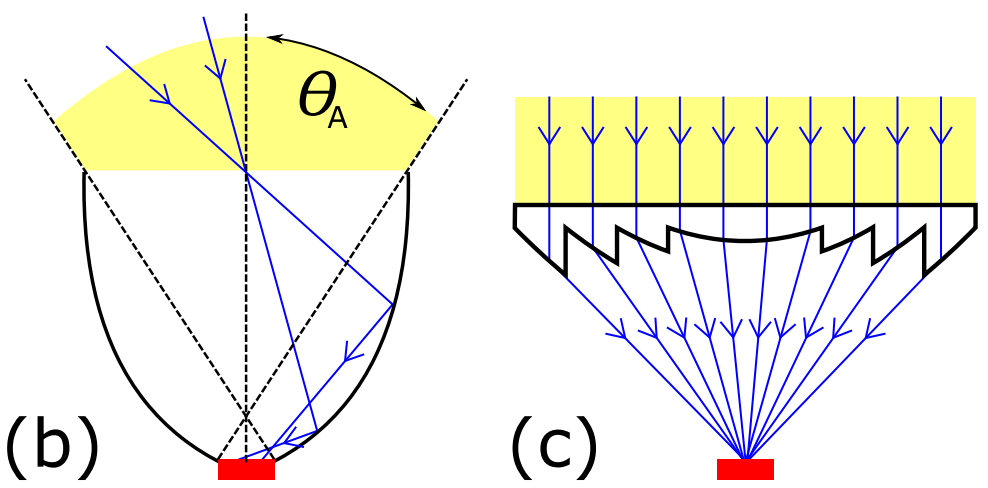

Figure 2.10: Various geometric concentrators: (a) parabolic, (b) compound parabolic, and (c) Fresnel lens. Blue: incoming solar rays; Yellow: allowed input angles; Red: PV cell. All three concentrators must track the sun, but only the compound parabolic can accept some diffuse light, so long as the incident angle is less than the acceptance angle $\theta_{\mathrm{A}}$.

optics have been demonstrated $[123,124]$. Numerous solar plants take advantage of solar concentrators. In the United States there are currently 26 large concentrated solar power projects, though none use PV cells to convert the sunlight to electricity [125].

\subsubsection{Maximum Concentration Factor}

The maximum concentration $C_{\max }$ for a geometric solar concentrator can be derived from either geometric or thermodynamic arguments, and is known as the sine brightness law [126-128]:

$$
\begin{aligned}
C_{\max , 2 \mathrm{D}} & =\frac{n \sin \theta_{\mathrm{E}}}{\sin \theta_{\mathrm{A}}}=\frac{n}{\sin \theta_{\mathrm{A}}} \\
C_{\mathrm{max}, 3 \mathrm{D}} & =\frac{n^{2} \sin \theta_{\mathrm{E}}}{\sin ^{2} \theta_{\mathrm{A}}}=\frac{n^{2}}{\sin ^{2} \theta_{\mathrm{A}}}
\end{aligned}
$$

where $C_{\max , 2 \mathrm{D}}$ and $C_{\max , 3 \mathrm{D}}$ are the maximum concentration for two and three dimensional concentrators, respectively, $\theta_{\mathrm{A}}$ is the maximum acceptance angle, $\theta_{E}=90^{\circ}$ is the maximum exit angle, and $n$ is the refractive index inside of the concentrator (assuming the exit plane is also at the same index).

Maximum concentration for two and three dimensional concentrators (equations 2.15 and 2.16) are plotted in figure 2.11, assuming the sun's angular size is $0.265^{\circ}$ full angle and 


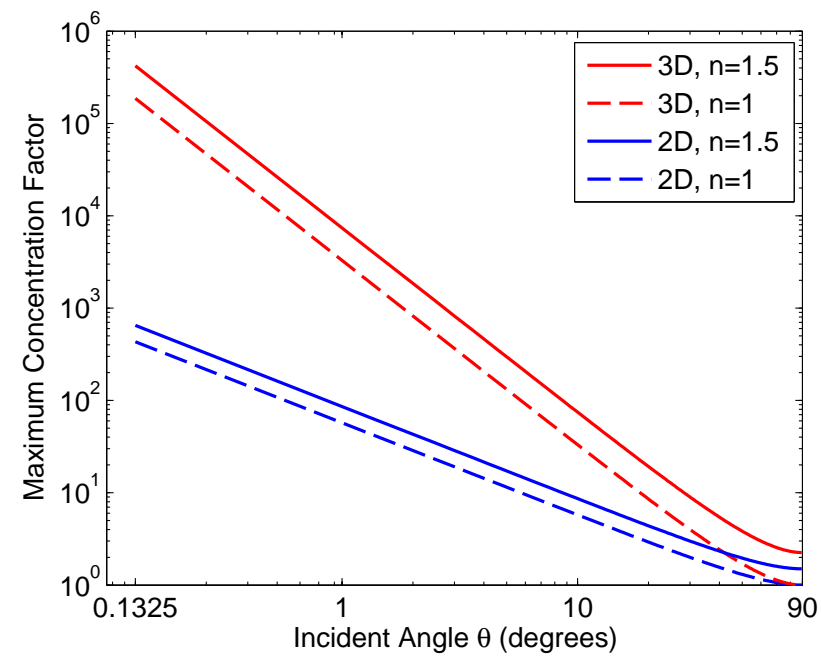

Figure 2.11: Geometric concentrator concentration factor verses acceptance angle $\theta_{\mathrm{A}}$. The plot starts at $0.1325^{\circ}$, the half angle of the sun's angular size. Three dimensional concentrators have a much higher maximum concentration, as expected, and increasing the index $n$ of the concentrator material also increases the maximum concentration which can be realized.

none of the sunlight is diffuse. Concentrators with $n=1$ and $n=1.5$ are shown. It is then easy to see why traditional concentrator systems must track the sun: at an acceptance angle of $\sim 1.3^{\circ}$, the concentration factor drops by an order of magnitude from $C_{\max }$. In the best case shown in figure $2.11, C$ is limited to 1000 at $\sim 2.7^{\circ}$.

\subsection{Luminescent Solar Concentrators}

LSCs operate on the principle of waveguiding, as discussed in section 1.1.1. Because their operating principle is different from geometric type concentrators, their losses and maximum concentration factor must be examined separately.

\subsubsection{LSC Optical Losses}

Front Surface Reflection and Escape Cone Loss

The optical losses in an LSC are shown in figure 2.12. The first two losses, front-surface reflection (FSR) and escape cone, are both related to the refractive index $n$ of the matrix 


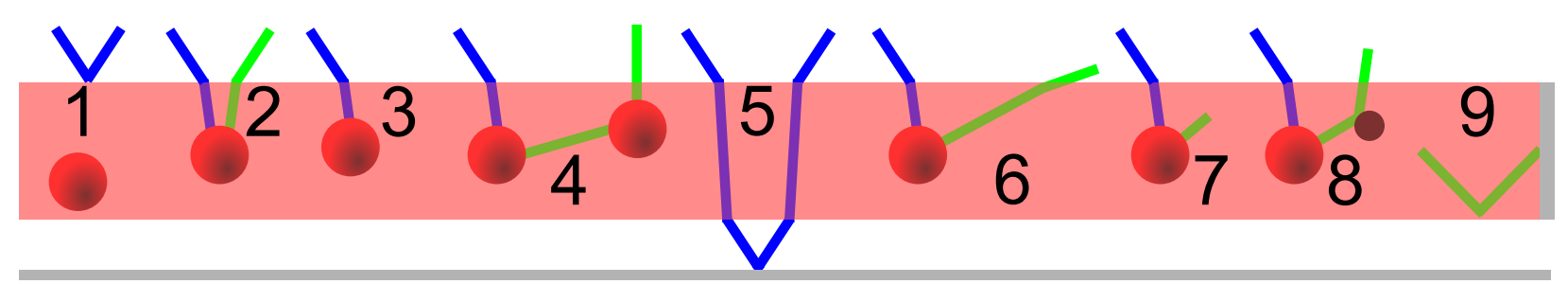

Figure 2.12: Optical losses in an LSC; incoming photons are blue, emitted photons are green, red dots are luminophores, gray surfaces are reflectors. (1) Front-surface reflection (FSR) (2) escape cone (3) QY loss (4) self absorption (5) unabsorbed radiation (6) TIR loss (7) matrix absorption (8) scattering (9) mirror loss.

material. FSR loss increases with $n$ according to the Fresnel equation. For $\mathrm{s}$ and $\mathrm{p}$ polarized light, reflectivity $R$ is given as:

$$
R_{\mathrm{s}}=\left|\frac{n_{1} \cos \theta-n_{2} \sqrt{1-\left(\frac{n_{1}}{n_{2}} \sin \theta\right)^{2}}}{n_{1} \cos \theta+n_{2} \sqrt{1-\left(\frac{n_{1}}{n_{2}} \sin \theta\right)^{2}}}\right|^{2} \quad R_{\mathrm{p}}=\left|\frac{-n_{2} \cos \theta+n_{1} \sqrt{1-\left(\frac{n_{1}}{n_{2}} \sin \theta\right)^{2}}}{n_{2} \cos \theta+n_{1} \sqrt{1-\left(\frac{n_{1}}{n_{2}} \sin \theta\right)^{2}}}\right|^{2}
$$

where $\theta$ is the angle of incidence ( $\theta=0$ for normal to the LSC surface), $n_{1}$ is the refractive index of the first medium and $n_{2}$ is the refractive index of the second medium. Reflection of unpolarized light can be calculated by taking the mean of $R_{\mathrm{s}}$ and $R_{\mathrm{p}}, R=\frac{1}{2}\left(R_{\mathrm{s}}+R_{\mathrm{p}}\right)$. Assuming normal incidence, FSR can be computed simply by:

$$
\mathrm{FSR}=\left(\frac{n_{1}-n_{2}}{n_{1}+n_{2}}\right)^{2}=\left(\frac{1-n}{1+n}\right)^{2}
$$

where $n_{1}=1$ is the refractive index of air and $n_{2}=n$ is the refractive index of the matrix.

For a cube or rectangular cuboid matrix, the escape cone is the solid-angle cone formed between an isotropic emitter and each face of the matrix, as shown in figure 2.13. In this cone, an emitted photon will not be captured via total internal reflection (TIR) $[129,130]$, as seen in figure 2.13. The cone has a half angle $\theta_{c}$, the critical angle, calculated with Snell's Law:

$$
\theta_{\mathrm{c}}=\arcsin \frac{1}{n}
$$




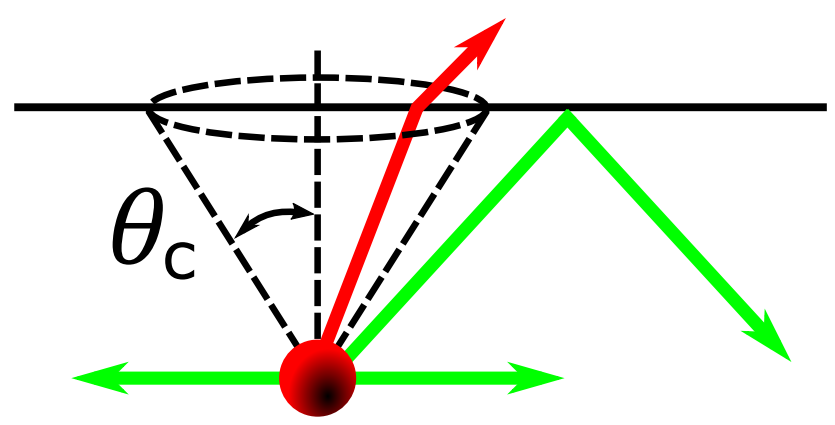

Figure 2.13: An illustration of the escape cone. The photons emitted at an angle greater than the critical angle $\theta_{c}$ (green lines) are guided. The photons emitted within the loss cone (red line) are not guided by TIR and are lost from the matrix.

The fraction of light $F_{\mathrm{EC}}$ captured by TIR in the matrix is the solid angle described by:

$$
F_{\mathrm{EC}}=\frac{\Omega_{\mathrm{ext}}}{4 \pi}
$$

where $\Omega_{\text {ext }}$ is the total solid angle exterior to the six escape cones (one cone for each face) and $4 \pi$ is the solid angle of a complete sphere.

Here, we are only concerned with two of the six cones, those facing up and down. The other four are the LSC edges and will either be mirrored or fitted with PV cells. $\Omega_{\text {ext }}$ is then the full solid angle minus the solid angle of two cones:

$$
\Omega_{\mathrm{ext}}=4 \pi-2 \Omega_{\mathrm{cone}} .
$$

The solid angle of a single loss cone $\Omega_{\text {cone }}$ is:

$$
\Omega_{\mathrm{cone}}=2 \pi\left(1-\cos \theta_{\mathrm{c}}\right)
$$

where $\theta_{\mathrm{c}}$ is the critical angle for TIR.

Combining equations 2.20, 2.21, and 2.22 yields the captured light fraction as a function of critical angle:

$$
F_{\mathrm{EC}}=\cos \theta_{\mathrm{c}} \quad \text { for } n \geq 1
$$


The loss from the pair of cones $L_{\mathrm{EC}}$ is then $\left(1-F_{\mathrm{EC}}\right)$, which can also be written in terms of matrix refractive index:

$$
L_{\mathrm{EC}}=1-F_{\mathrm{EC}}=1-\cos \theta_{\mathrm{c}}=1-\frac{\sqrt{n^{2}-1}}{n}
$$

by taking advantage of the identities $\theta_{c}=\arcsin \frac{1}{n}$ and $\cos \left(\arcsin \frac{1}{n}\right)=\sqrt{1-\left(\frac{1}{n}\right)^{2}}$. Thus, loss cone will decrease with increasing $n$ because TIR increases.

Adding equations 2.18 and 2.24, plotted in figure 2.14 as a function of $n$, the ideal matrix index can be found: $n=1.985$, with a loss of $24.51 \%$. At the index of many polymers and glasses, $n=1.5$, the loss increases only slightly to $29.46 \%$. This is a bit simplistic, however, as it does not account for the effect of a bottom mirror to reflect back photons lost from the downward escape cone.
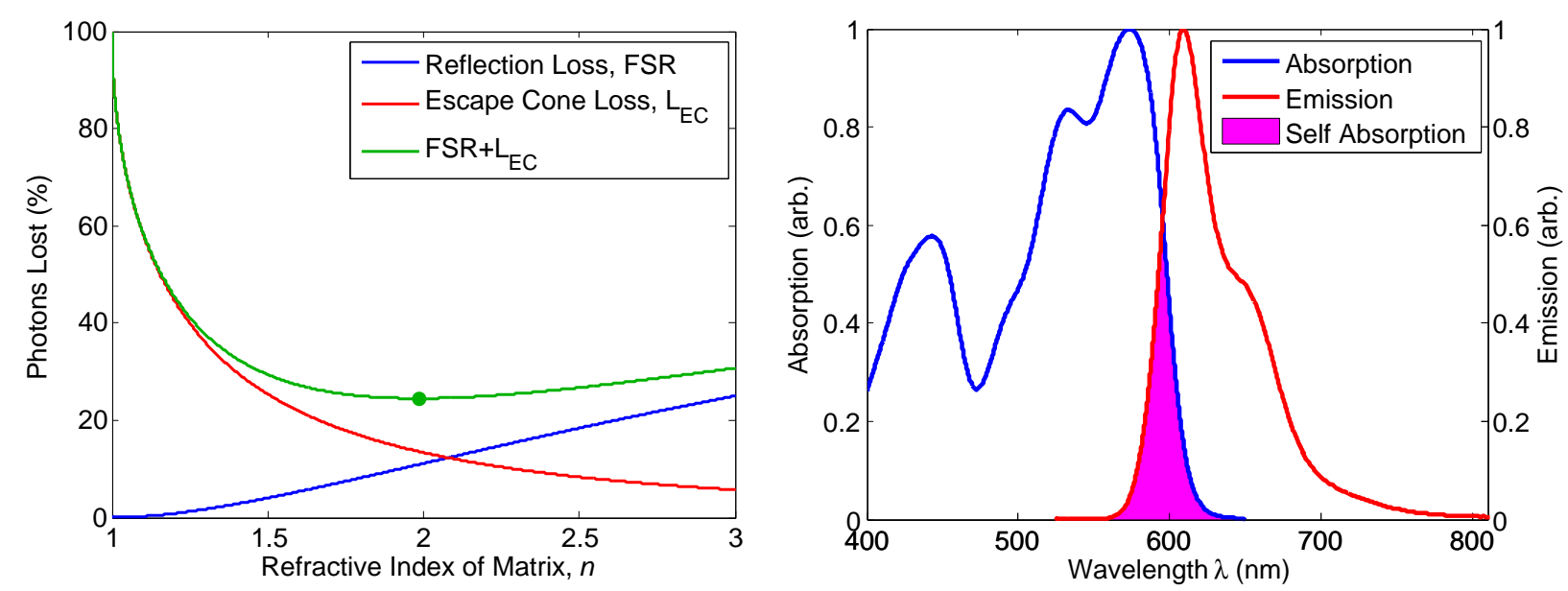

Figure 2.14: Effect of matrix material in- Figure 2.15: An example of self absorption dex on optical losses. Total loss is a combi- in a luminophore. Where the absorption and nation of reflection and escape cone losses. emission spectra overlap for Red 305, there is poThe minimum loss occurs at an index of tential for self absorption, shown as the shaded 1.99 , shown as the green dot.

region.

\section{Quantum Yield Loss}

The third loss shown in figure 2.12 is from $Q Y<1$. Any time a photon is absorbed, there is a chance, given by $1-$ QY, that it will be lost to heat through a non-radiative decay process. 


\section{Self Absorption}

The fourth loss, due to self-absorption, is related to the escape cone and QY losses. Most luminophores do not have completely separated absorption and emission spectra and are able to re-absorb a photon previously emitted by another luminophore, as shown in figure 2.15 for Red 305 dye. This is another opportunity for the photon to be lost to the escape cone or QY losses because it is either emitted in a random direction or lost as heat.

\section{Unabsorbed Radiation}

Due to self absorption, the luminophore concentration must be limited below the level at which it fully absorbs the sun's rays. A balance must be struck between self absorption loss and the fifth loss, unabsorbed radiation. Unabsorbed radiation passes completely through the LSC twice (once after being reflected from the back reflector) without being absorbed. This can be a result of the photon being outside of the absorption band of the luminophore entirely, or the luminophore concentration being too low to fully absorb the incoming radiation. A diffuse backside reflector will help to limit unabsorbed radiation, as it will increase the path length back through the LSC, as well as scatter some incoming photons directly toward the attached PV cells.

\section{Total Internal Reflection Loss}

The sixth loss is TIR loss. Since a practical surface is never perfectly planar, there is a small chance that guided photons will be lost with every bounce instead of reflecting with 100\% certainty. For an acrylic sample, it was found that the chance for a photon to be lost from the LSC on each bounce is between $0.04 \%$ and $\geq 2 \%$ [131]. The loss per bounce could be lowered from $2 \%$ loss per bounce to $\sim 0.67 \%$ by cleaning the acrylic, as losses were caused by dust and other aerosol particulates on the surface. However, it took careful preparation, cleaning, and special coating to reach $0.04 \%$ loss per bounce. At this limit, the losses were caused by non-uniformity in the surface, which showed "significant micro-structure" in the 
$0.1-1 \mu \mathrm{m}$ range. The loss per bounce $L_{\mathrm{b}}$ is proportional to [131]:

$$
L_{\mathrm{b}} \propto \frac{T(n-1)^{2}}{\lambda^{2} \gamma \cos \theta}
$$

where $T$ is absolute temperature, $n$ is the matrix refractive index, $\lambda$ is the photon wavelength in vacuum, $\gamma$ is the matrix surface tension, and $\theta$ is the angle of incidence to the LSC surface. Here $\gamma$ is used as a way of approximating surface roughness through the relation:

$$
\gamma \approx \frac{k_{\mathrm{B}} T}{\pi \sigma^{2}}
$$

where $k_{\mathrm{B}}$ is Boltzmann's constant, $T$ is absolute temperature, and $\sigma$ is the peak height of a circle segment protruding above the matrix surface plane, as seen in figure 2.16.

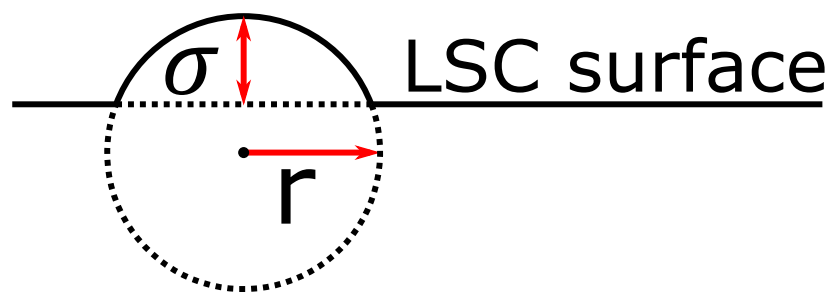

Figure 2.16: An illustration of the approximation for surface roughness used in finding the loss per bounce. $\sigma$ is the peak height of the circle segment above the surface plane and $r$ is the circle radius.

\section{Matrix absorption}

Since a real matrix will never be completely transmissive, there is also a chance for photons to be absorbed by the matrix and be lost as heat, shown as the seventh loss. Many "clear" polymers are very transmissive in the visible region, but absorb heavily in the UV and slightly in the near IR regions. UV absorption is caused by electronic transitions from the highest occupied molecular orbital (HOMO) to the lowest unoccupied molecular orbital (LUMO) in the polymer, or additional impurities [132]. If an azo type initiator (such as azobisisobutyronitrile) is used for polymerization and any is left unreacted, for example, it 
can absorb with a peak near $360 \mathrm{~nm}$. Hydroquinone, a polymerization inhibitor used for stabilizing many monomers during storage, can also be a problem, as it forms p-benzoquinone through oxidation; p-benzoquinone has a UV absorption peak which can tail into the visible spectrum. Since sunlight at Earth's surface has very few UV photons, this is a fairly negligible loss.

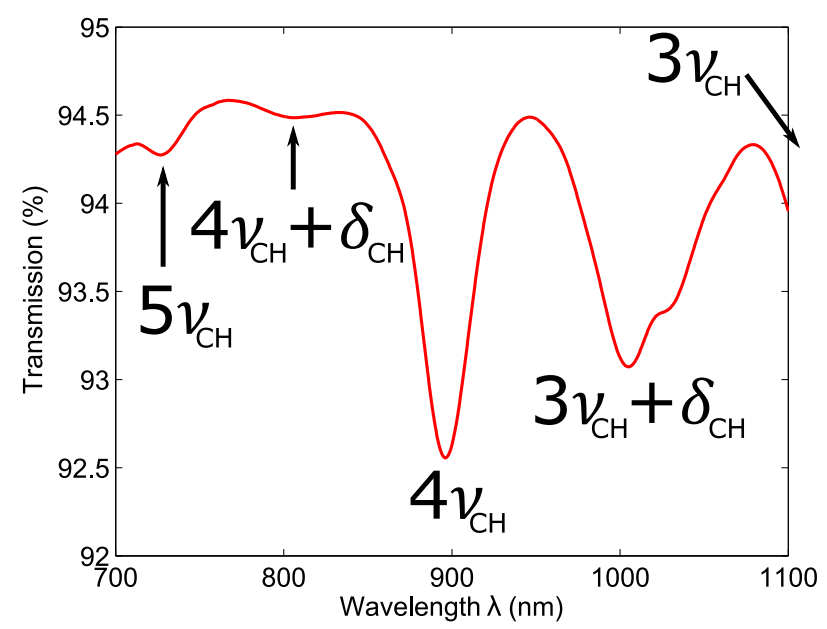

Figure 2.17: An example of near IR absorption in a polymer. Absorption modes are shown for PMMA, seen as dips in the transmission spectra verses wavelength. Transmission curve measured and matched to data in literature [132]. The $3 \nu_{\mathrm{CH}}$ absorption peak occurs near $1200 \mathrm{~nm}$, but its tail can be seen near $1100 \mathrm{~nm}$.

Near IR absorption is a larger problem, as this can absorb photons emitted by certain luminophores. This absorption, which actually ranges into the visible, is caused by harmonics of the carbon-hydrogen $(\mathrm{CH})$ bond molecular stretching and bending mode vibrations [132]. Stretching mode vibrations, $\nu_{\mathrm{CH}}$, cause optical absorption at many frequencies. These frequency bands are harmonics of the fundamental absorption frequency at $\lambda \approx 3.3 \mu \mathrm{m}$, and are noted as $k \nu_{\mathrm{CH}}$, where $k$ is the integer vibrational quantum number, the $k^{\text {th }}$ harmonic. Bending mode vibrations, $\delta_{\mathrm{CH}}$, cause optical absorption at an offset from each stretching mode harmonic, and so are noted $k \nu_{\mathrm{CH}}+\delta_{\mathrm{CH}}$. With each increase in $k$, the absorption intensity of the peak at that harmonic is decreased by about one order of magnitude. The near IR absorption peaks for an example polymer (PMMA) can be seen in figure 2.17. It is also possible for many polymers to absorb water in humid environments, in which case 
certain harmonics of the oxygen-hydrogen bond (fundamental at $\lambda \approx 2.8 \mu \mathrm{m}$ ) will absorb in the visible and near IR spectrum.

\section{Scattering}

Scattering is the eighth loss shown in figure 2.12. When a photon is scattered, it will at least have a longer path length before making it to a PV cell, increasing its chances of being self absorbed. At worst, it will be directed at an angle which will not waveguide, and the photon is likely to be lost nearly immediately.

Scattering can occur because of some extrinsic property, such as micro-cavities or large impurities resulting from the casting process, or some intrinsic property, such as fluctuations in polymer density (and therefore refractive index) or fluctuations in luminophore density, with Rayleigh scattering being the lower limit for a "perfect" material [131-133].

\section{Mirror Loss}

The ninth loss is a result of mirrors with a reflectance $R<1$, where the probability of losing the photon is $1-R$ at each reflection from a mirror. This loss is minor and can be minimized by using readily available high quality reflectors. $100 \%$ polymer films are commercially available with $R \geq 0.98$ in the visible and near IR regions which are ideal for LSC use [134]. These films are multilayer dielectric stacks which take advantage of alternating layers of birefringent and non-birefringent polymers to maintain high reflectance across a broad wavelength spectrum and high incident angle [135].

\section{LSC Scaling}

When an LSC is scaled to various sizes, loss mechanisms scale differently. FSR, escape cone, QY loss, unabsorbed radiation, and mirror loss are unaffected by the length or width of the LSC. Self absorption losses will scale to an extent, and as the LSC gets longer or wider, the optical efficiency is diminished. However, this effect quickly saturates. After a relatively short 
propagation distance through the matrix, proportional to the concentration of luminophore, all of the photons which can be self absorbed have been lost.

Another group of losses, which can be collectively called "transport losses," scale with LSC size and will cause the optical efficiency to drop for larger LSCs. These losses include matrix absorption, scattering, and TIR loss. These loss mechanisms operate on guided photons of all wavelengths and continually diminish their number as they are guided through the matrix. The total transport loss in PMMA was estimated to be $\sim 1-3 \% / \mathrm{cm}$ for $\lambda \approx 633$, and represents the ultimate upper limit to practical optical concentration in an LSC [131], discussed in section 2.4.2.

\subsubsection{LSC Concentration Limits}

Unlike standard geometric type concentrators, a ray optic approach cannot be used when determining the concentration limit $C_{\max }$ of an LSC because photon energy, and thus flux, is not conserved.

Instead, it can be determined by considering entropy change. This analysis was originally performed by Yablonovitch [136]. The change in entropy $\Delta S_{1}$ from a photon being lost from the incident light field (i.e. being absorbed by the LSC) is:

$$
\Delta S_{1}=-k_{\mathrm{b}} \ln \left(1+\frac{8 \pi n^{2} \nu_{1}^{2}}{c^{2} B_{1}}\right)
$$

and the entropy change $\Delta S_{2}$ in the concentrated light field (i.e. emitted by the LSC) is:

$$
\Delta S_{2}=k_{\mathrm{b}} \ln \left(1+\frac{8 \pi n^{2} \nu_{2}^{2}}{c^{2} B_{2}}\right)+\frac{h\left(\nu_{1}-\nu_{2}\right)}{T}
$$

where $k_{\mathrm{b}}$ is Boltzmann's constant, $n$ is the refractive index of the LSC, $B_{1}$ and $B_{2}$ are the brightness of the incident and concentrated fields, $\nu_{1}$ and $\nu_{2}$ are the frequency of the incident and emitted photon, $c$ is the speed of light, $h$ is Planck's constant, and $T$ is absolute 
temperature. The second term of equation 2.27 is the thermal dissipation of energy due to the Stokes shift $\Delta \nu$.

The second law of thermodynamics requires $\Delta S_{1}+\Delta S_{2} \geq 0$. Then, combining equations 2.26 and 2.27:

$$
\frac{\left(1+\frac{8 \pi n^{2} \nu_{2}^{2}}{c^{2} B_{2}}\right)}{\left(1+\frac{8 \pi n^{2} \nu_{1}^{2}}{c^{2} B_{1}}\right)} \leq \exp \left[\frac{h\left(\nu_{1}-\nu_{2}\right)}{k_{\mathrm{b}} T}\right] .
$$

Since $\nu^{2} / c^{2}$ is large for visible wavelengths:

$$
\frac{8 \pi n^{2} \nu^{2}}{c^{2} B} \gg 1
$$

the maximum concentration can be found as the ratio of output to incident brightness:

$$
C_{\max } \equiv \frac{B_{2}}{B_{1}} \approx \frac{\nu_{2}^{2}}{\nu_{1}^{2}} \exp \left[\frac{h\left(\nu_{1}-\nu_{2}\right)}{k_{\mathrm{B}} T}\right]=\frac{\lambda_{1}^{2}}{\lambda_{2}^{2}} \exp \left[\frac{h c\left(\lambda_{1}^{-1}-\lambda_{2}^{-1}\right)}{k_{\mathrm{B}} T}\right]
$$

where $\lambda_{1}$ and $\lambda_{2}$ are the wavelength of the incident and emitted photons.

It is easy to see from equation 2.29 that $C_{\max }$ is highly sensitive to Stokes shift and temperature. This is shown in figures 2.18 and 2.19. For the example dye shown in figure $2.15, \lambda_{1}=573.5 \mathrm{~nm}$ and $\lambda_{2}=609.5 \mathrm{~nm} . C_{\max }$ is then 139 at $20^{\circ} \mathrm{C}$ and 201 at $0^{\circ} \mathrm{C}$. Setting $\lambda_{1}=550 \mathrm{~nm}$ and $\lambda_{2}=650 \mathrm{~nm}$ for a modest improvement in Stokes shift results in $C_{\max }=6.4 \times 10^{5}$ at $20^{\circ} \mathrm{C}$.

Based only on a thermodynamics argument, there is potential for massive concentration factors with LSCs. Practically, however, there are many limits and losses (discussed in section 2.4.1), and such high Stokes shifts are rare or impossible for luminophores. The limiting factor for $C_{\max }$, then, is not thermodynamics, but the losses inherent to realistic materials. One study concluded that concentrations up to about 1000 should be possible using a realistic luminophore, based on their measurements of transport losses and predictions of how much lower these losses could reasonably be made [131].

There is also the trade-off between high $C_{\max }$ and the ability to absorb more of the solar 


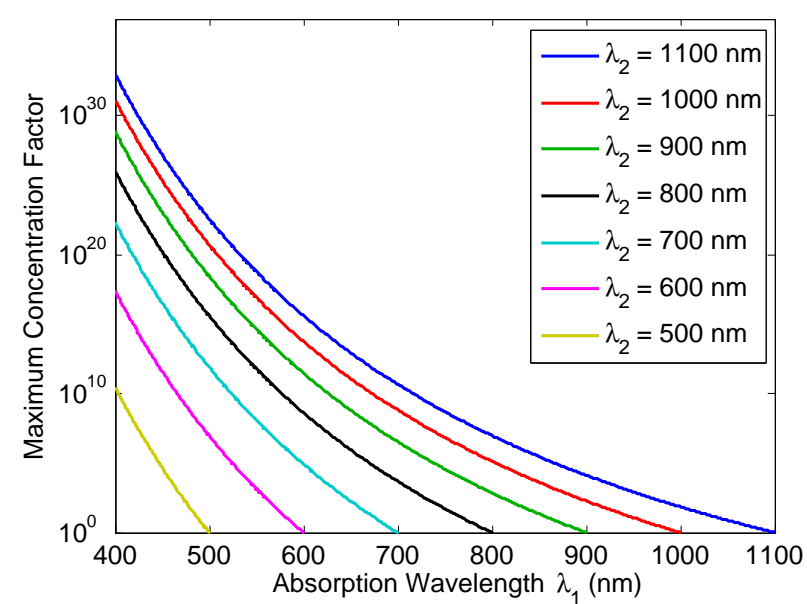

Figure 2.18: Maximum concentration factor $C_{\max }$ for an LSC containing a luminophore of various emission wavelengths $\lambda_{2}$, plotted against absorption wavelength $\lambda_{1}$. A higher Stokes shift $\Delta \lambda$ results in a higher $C_{\max }$.

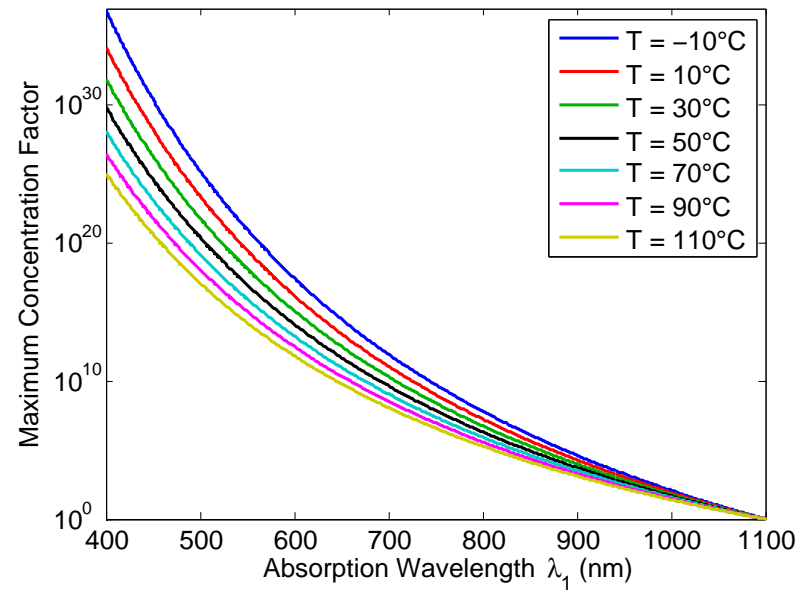

Figure 2.19: Maximum concentration factor $C_{\max }$ for an LSC containing a luminophore with $\lambda_{2}=1100$ plotted against absorption wavelength $\lambda_{1}$. Each trace is a different ambient temperature; as the temperature rises, $C_{\text {max }}$ falls.

spectrum. A luminophore that only absorbs below $400 \mathrm{~nm}$ and emits at $1100 \mathrm{~nm}$, for example, would have an incredibly high potential $C$. But, it would be nearly useless for harvesting solar energy. A luminophore instead matched to a Si PV cell with $\lambda_{1}=910 \mathrm{~nm}$ and $\lambda_{2}=1050 \mathrm{~nm}$ would absorb nearly all of the solar spectrum usable by the PV cell while still having a $C_{\max } \approx 1000$.

\subsection{Polymerization of Matrix Material}

While there are many potential methods of incorporating luminophores into a polymer, this work involves mixing the luminophore with a liquid monomer solution and curing the mixture in a mold. Many techniques are available, but the most common is based on free radical vinyl polymerization [137-140]. All of the monomers used in this work are vinyl type monomers.

As shown in figure 2.20 a, the first step in polymerization is the breakdown of a radical initiator into free radicals, a molecule with an unbound electron which wants to form a covalent bond. Azobisisobutyronitrile (AIBN) is pictured in this example. AIBN decomposes 
when heated to form two free radical molecules and one nitrogen molecule. Other initiators, such as bis(2,4,6-trimethylbenzoyl)-phenyl phosphine oxide, decompose to form free radicals when a UV photon is absorbed by the molecule.

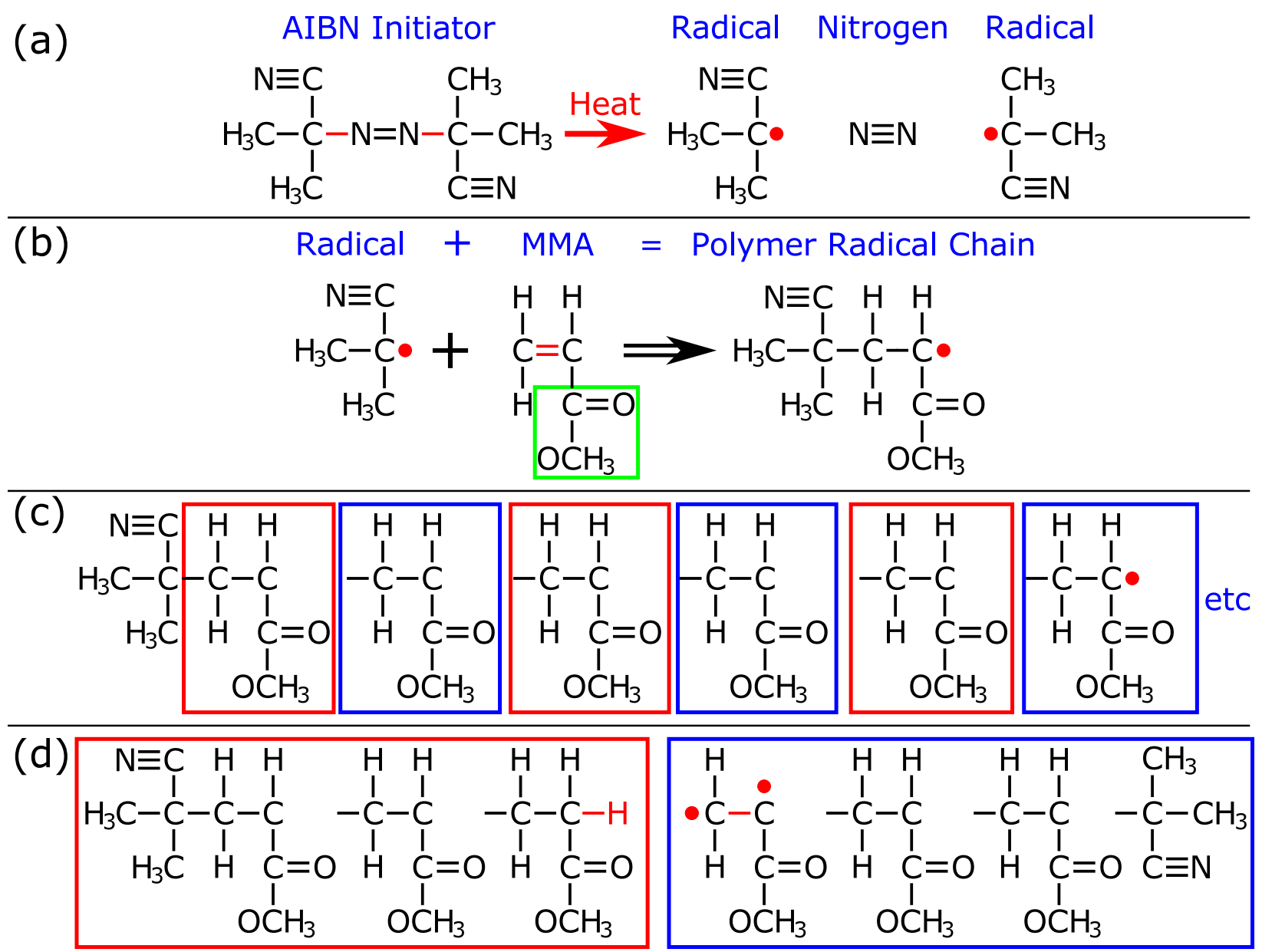

Figure 2.20: An example of polymerization. Bonds (covalent electron pairs) are shown as bars, unbound electrons as circles. Important bonds or unbound electrons are shown in red. (a) Thermal decomposition of the azo type radical initiator AIBN into two radical molecules and one $\mathrm{N}_{2}$ molecule. (b) The radical interacts with an MMA monomer unit, forming a polymer radical chain. The chemical group shown in green is what differentiates MMA from many other vinyl type monomers, having replaced a hydrogen atom on the carbon backbone. (c) The polymer radical chain interacts with additional monomer units, forming a longer chain. Repeat units are highlighted in alternating red and blue. (d) Chain termination by disproportionation. The first chain is highlighted red, the second blue.

In the second step (figure 2.20b), the free radical reacts with a monomer, methyl methacrylate (MMA) in this example. The double bond between the carbon atoms $(\mathrm{C}=\mathrm{C})$ is broken, creating free electrons on both of these carbon atoms. The carbon atom on the radical 
molecule then forms a single bond with one of the carbons on the MMA, leaving a free electron on the other MMA carbon. The chain is now a free radical, and will continue to react with additional MMA units to form a longer polymer chain, as shown in $2.20 \mathrm{c}$.

The chain will continue its growth until it is terminated. This can happen in one of two ways: coupling or disproportionation. Coupling happens when the radical ends of two polymer chains react and join together, forming a single long chain. Disproportionation is shown in figure $2.20 \mathrm{~d}$. When the radical end of one chain gets near to the radical end of another chain, sometimes the unbound electron of chain one will remove and bond with a hydrogen atom from one of the carbon atoms near to the radical end of chain two. Chain one is then terminated. Chain two can then terminate by forming a double bond between the two carbon atoms on the end of the chain, or a branched chain can form if both radical sites react with other chains. Branching is relatively rare in PMMA.

Polymerization of AB9093, a proprietary UV curing epoxy, follows very similarly to the above example of PMMA, as does lauryl methacrylate (LMA). When ethylene glycol dimethacrylate (EGDMA) is added to LMA, a copolymer is formed, meaning all the repeat units in the chain will not be identical. Additionally, EGDMA has two active sites instead of the single active site of MMA or LMA, so the polymer chains become a heavily branched network. It is for this reason EGDMA is incorporated into the LMA solution: the heavily branched network of polymer chains forms a much stiffer polymer than the naturally soft, almost gelatinous PLMA.

Oxygen must not be present during vinyl radical curing. Oxygen will scavenge free radicals, bonding with them and prematurely terminating polymer chains or preventing the initiator-created radical molecules from initiating polymerization altogether. Monomers are typically sold and shipped with an inhibitor molecule such as hydroquinone (HQ) or monomethyl ether of hydroquinone (MEHQ) and packed under oxygen to prevent spontaneous polymerization.

The process of vinyl radical polymerization is exothermic, so the sample temperature 
must be managed. This is an especially large problem when using thermal initiators, as a runaway reaction can result: higher temperatures lead to greater numbers of free radicals which then generate more heat, and so on. 


\section{Chapter 3: LSC Modeling}

\subsection{Background}

A Monte Carlo ray tracing model was implemented in MATLAB; source code is available in appendix B. The model examines the path taken by many individual photons through an LSC and records the result until convergence is reached. By examining where each

photon terminates and how it got there, the predicted optical efficiency $\eta_{\text {opt }}$ of the LSC and magnitude of each loss can be determined. For example, a photon which makes it to a solar cell will count toward the $\eta_{\mathrm{opt}}$ of the device. If the photon is absorbed by a luminophore and immediately lost to the escape cone, this is recorded as a escape cone loss, etc. $\eta_{\text {opt }}$ is here defined as the ratio of input photons (including those lost to surface reflection before entering the LSC) to output photons, irrespective of photon energy. The simulation can be run with many user-settable inputs including arbitrary luminophore and light source spectra, so it is robust and powerful for use in a number of scenarios.

User-selectable input parameters to the model and their default values are listed below:

- rectangular LSC dimensions of $20.8 \mathrm{~mm} \times 20.8 \mathrm{~mm} \times 5.2 \mathrm{~mm}$,

- matrix material: P(LMA-co-EGDMA),

- refractive index $n=1.4545$

- absorbance as a function of wavelength, as shown in figure 3.1

- luminophore: PbSe QDs,

$-\mathrm{QY}=0.7$

- absorbance and fluorescence intensity as a function of wavelength, as shown in figure 3.2

- variable relative concentration

- mirrors, 
- specular side mirrors, $R=0.97$ on two opposite LSC edges, no air gap

- diffuse bottom reflector, $R=0.97$, reflects at a Lambertian random angle, with air gap

- without wavelength-selective top mirror

- PV cells on opposite edges of the LSC,

$-V_{\mathrm{oc}}=0.55 \mathrm{~V}$ (measured)

$-\mathrm{EQE}=0.8$ (estimated)

$-\mathrm{FF}=0.7$ (measured)

- 22\% efficiency under one sun AM1.5 illumination (manufacturer's specification)

- wavelength at the band gap $\lambda_{\mathrm{g}}=1100 \mathrm{~nm}$

- dielectric stack between the LSC and PV cell consisting of the following layers:

- LSC matrix material, infinite thickness

- AB9093 bond layer, $150 \mu \mathrm{m}$ thick, $n=1.56$

- silicon nitride anti-reflection coating, $72.5 \mathrm{~nm}$ thick [141], $n=2.0164$

- silicon dioxide passivation layer, $20 \mathrm{~nm}$ thick, $n=1.4754$

- silicon PV cell, infinite thickness, $n=3.9609$

- Thorlabs OSL1 fiber-optic illuminator halogen source with normal incident angle and random starting position, as shown in figure 3.3,

- TIR loss per bounce $L_{\mathrm{b}}=0.015$,

- photon count $2 \times 10^{6}$ (typically $\geq 5 \times 10^{5}$ photons will show convergent results).

Refractive indices above are given at the $\mathrm{D}_{1}$ Fraunhofer line, $\lambda \approx 590 \mathrm{~nm}$, at $20^{\circ} \mathrm{C}$ or $25^{\circ} \mathrm{C}$, depending on the source. Within the simulation, real values of refractive index are given as a function of wavelength, as shown in figure 3.4. Wavelength dependent data for silicon [142,143], silicon dioxide [144,145], silicon nitride [146], and PMMA [147] were found in literature and interpolated as necessary. Data at only one wavelength could be found for AB9093 [148], PLMA [149-152], and EGDMA [150,153-155]. For AB9093, the PMMA curve was shifted up so that it was equal to the $n$ of AB9093 at $\lambda=590 \mathrm{~nm}$. For 


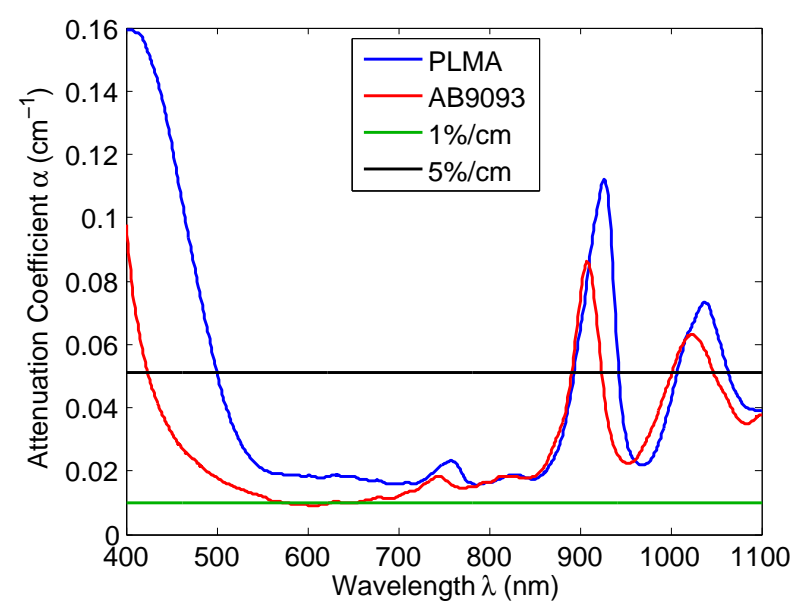

Figure 3.1: Attenuation coefficients as a function of wavelength for various matrices examined in this work. Measured values for P(LMA-co-EGDMA) and AB9093 are used, as well as a flat absorbance of $1 \% / \mathrm{cm}$ and $5 \% / \mathrm{cm}$.

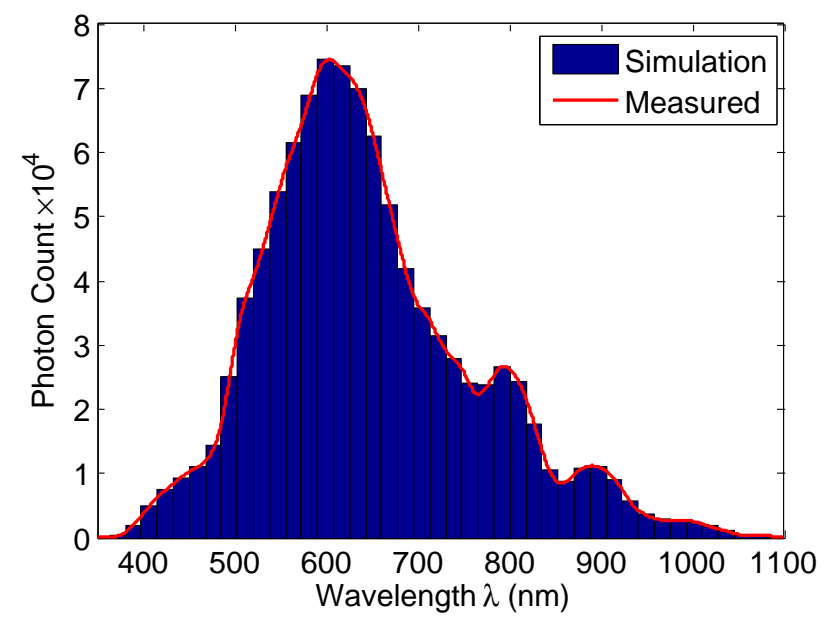

Figure 3.3: Histogram (blue) of the input photons' wavelength used in the simulation for an OSL1 lamp source (50 bins, one million photons). Photons are created at a certain wavelength with a probability proportional to the height of the red curve (experimentally measured) at that wavelength. In this case, the red curve is the measured photon counts of the OSL1 lamp.

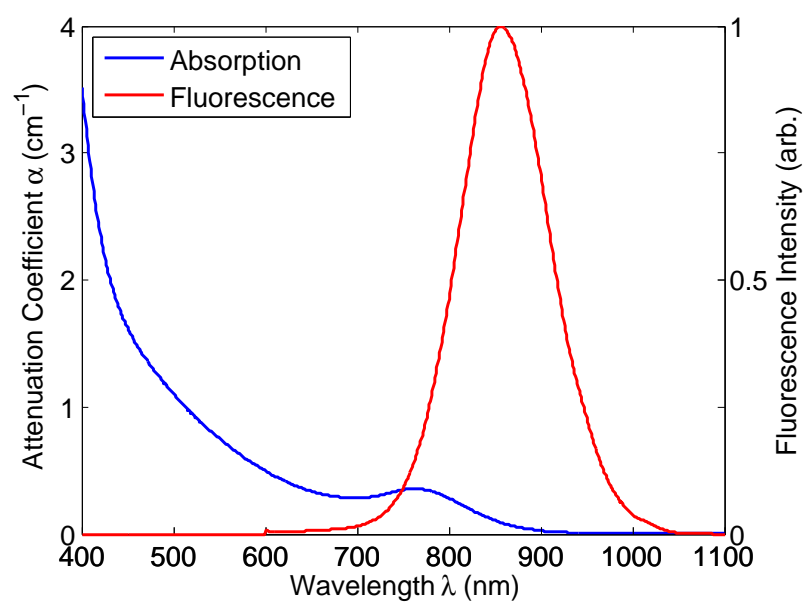

Figure 3.2: Attenuation coefficients and fluorescence intensity as a function of wavelength for the QDs used in the simulation. Originally measured in a $\mathrm{P}$ (LMA-co-EGDMA) matrix, which has very little change from QDs in hexane.

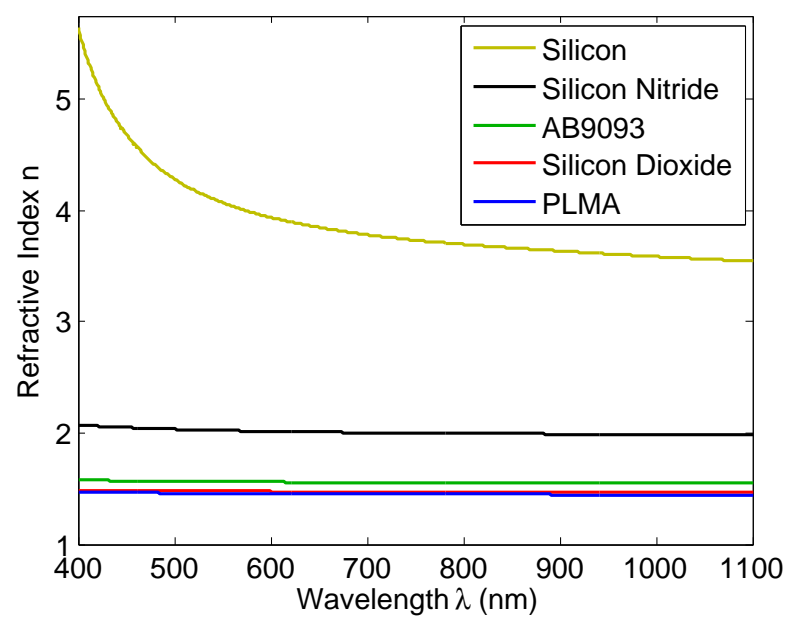

Figure 3.4: Refractive indices as a function of wavelength for various materials used in the simulation. Note: P(LMA-co-EGDMA) and silicon dioxide are difficult to distinguish visually on the plot because of their similar values. 
P(LMA-co-EGDMA), a weighted average of LMA and EGDMA values was taken, and the PMMA curve shifted down to match this value at $\lambda=590 \mathrm{~nm}$.

As a photon propagates through the matrix, it will travel along its current direction until it hits an LSC face or is absorbed. The program calculates a travel distance dist trav $_{\text {before }}$ absorption based on the inverse cumulative density function (CDF) of the Beer-Lambert Law $[156,157]$ :

$$
\text { dist }_{\mathrm{trav}}=\frac{-\ln (\xi)}{\alpha_{\mathrm{QD}} N+\alpha_{\mathrm{mat}}}
$$

where $\xi$ is a random number in the range [0 to 1$]$ with a uniform distribution, $\alpha_{\mathrm{QD}}$ and $\alpha_{\mathrm{mat}}$ are the absorption coefficient of the QD and matrix (calculated from user input) and $N$ is the relative concentration of QDs. If this calculated distance is less than the distance to the next LSC face, the photon is absorbed. Its location is then its current location plus the calculated distance along its travel direction:

$$
\begin{aligned}
& x^{\prime}=x+d i s t_{\mathrm{trav}} \cos \phi \sin \theta \\
& y^{\prime}=y+d i s t_{\mathrm{trav}} \sin \phi \sin \theta \\
& z^{\prime}=z+d i s t_{\mathrm{trav}} \cos \theta
\end{aligned}
$$

where $x, y$, and $z$ are the previous photon coordinates, $x^{\prime}, y^{\prime}$, and $z^{\prime}$ are the new photon coordinates, $\phi$ is the azimuthal angle, and $\theta$ is the polar (a.k.a deflection or zenith) angle. $\phi$ is in the range $(-\pi$ to $\pi$ ] where $\phi=0$ is in the positive $x$ direction and $\phi=\pi / 2$ is in the positive $y$ direction. $\theta$ is in the range $[0$ to $\pi]$, where 0 is normal to the LSC plane in the positive $z$ direction (pointed up). If dist $t_{\text {trav }}$ is greater than the distance to the next face, dist $_{\text {trav }}$ becomes the distance to the face.

If absorbed, the simulation must then decide whether the photon was absorbed by the matrix or by a QD. This is done with a simple ratio of absorption probabilities. The 
probability of the photon being absorbed by the matrix is:

$$
\mathrm{P}(\text { matrix absorption })=\frac{1-\exp \left[-\alpha_{\text {mat }} \text { dist }_{\text {trav }}\right]}{\left(1-\exp \left[-\alpha_{\text {mat }} d i s t_{\text {trav }}\right]\right)+\left(1-\exp \left[-\alpha_{\mathrm{QD}} N d i s t_{\text {trav }}\right]\right)}
$$

and the probability of the photon being absorbed by the luminophore is:

$$
\mathrm{P}(\text { luminophore absorption })=1-\mathrm{P}(\text { matrix absorption })
$$

Fluorescence events are triggered when the photon is absorbed by a luminophore. Fluorescence will occur with a probability equal to the QY value set by the user (i.e. $\xi \leq \mathrm{QY}$ ). If fluorescence takes place, it will only occur at a random wavelength greater than or equal to the wavelength of the absorbed photon subject to the CDF of the emission spectrum.

A sample emission spectra and its CDF are shown in figure 3.5. If the wavelength of the absorbed photon is shorter than the emission spectrum, a random number $\xi$ is chosen and compared to the CDF. The new fluorescence wavelength is the wavelength where the CDF matches the random number. If the current wavelength is within the emission spectrum, the CDF must be shifted to guarantee the fluorescence wavelength is always longer than the current wavelength; it is shifted further for longer excitation wavelengths. This simulates QD emission behavior at long excitation wavelengths [158] without the need to measure the excitation wavelength-dependent QD emission. Because of the way fluorescence wavelengths are chosen, the LSC output spectral shape will only be accurately predicted for single-peak emission spectra (see for example figure 2.15).

The photon is assigned the new fluorescence wavelength and a random isotropic travel direction angle pair at its current position upon completion of the fluorescence event. The direction, specified by $\phi$ and $\theta$, is assigned as [159,160]:

$$
\begin{aligned}
& \phi=2 \pi \xi_{1} \\
& \theta=\arccos [\operatorname{sign}(\chi)-\chi] \quad \chi=2 \xi_{2}-1
\end{aligned}
$$


where $\xi_{1}$ and $\xi_{2}$ are random numbers in the range [0 to 1$]$ with uniform distribution.

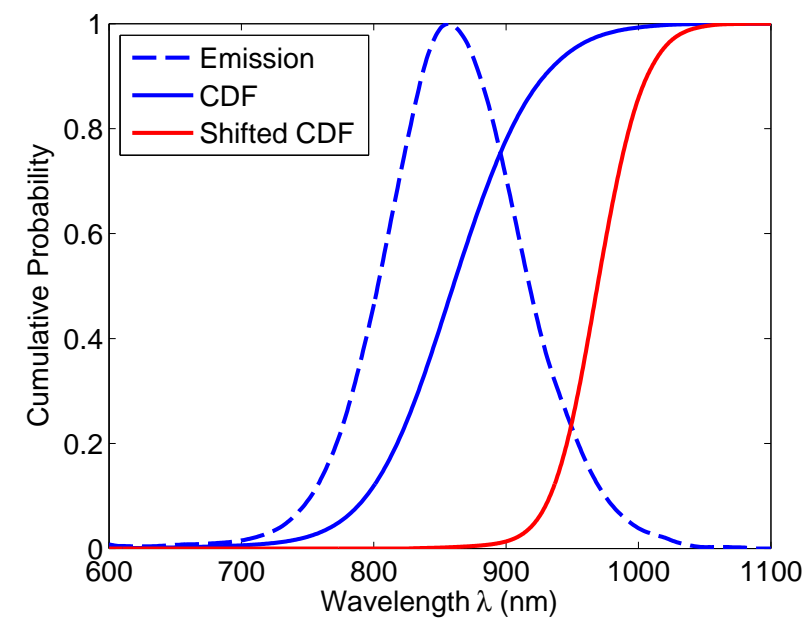

Figure 3.5: An example QD emission spectra and its cumulative density function for modeling fluorescence wavelength. The red line has been shifted to ensure the emission is at a longer wavelength than the excitation wavelength.

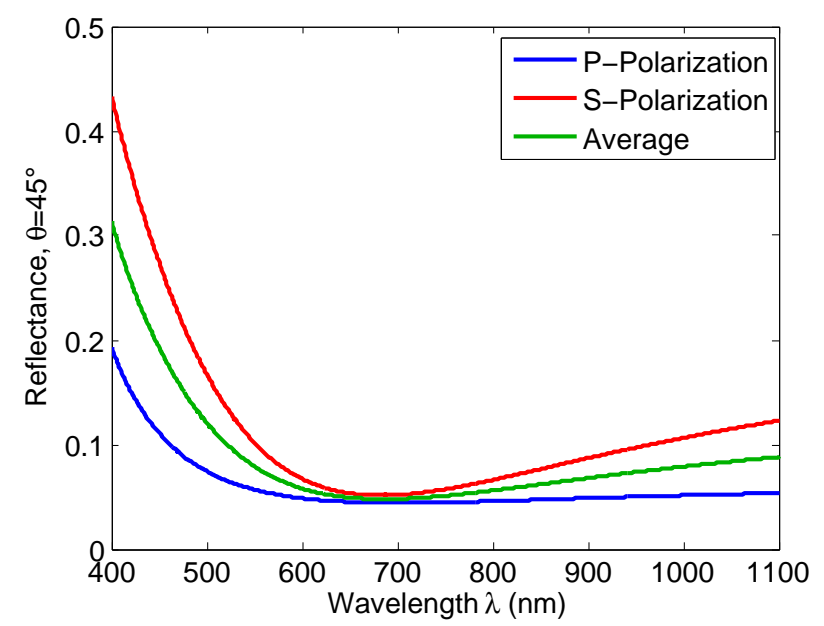

Figure 3.6: Reflectance from the PV cell surface at $\theta=45^{\circ}$ including the effects of the dielectric stack between the LSC and cell. Reflection probability of photons with p- and s- polarizations are calculated and averaged.

Propagation of each photon continues until it is output to a PV cell or lost, and its final location is recorded for later analysis. If the photon is not absorbed before reaching a face, a reflection probability must be computed and compared to $\xi$ to see if the reflection is successful. For mirrors, this probability is set by the user. For single interface dielectric reflections, Fresnel equations (equation 2.17) are used. A photon polarization is randomly chosen at the time of the reflection event for the purposes of computation, and TIR loss $L_{\mathrm{b}}$ is subtracted from the computed probability. Since there is an air gap between LSC and the bottom mirror, the photon undergoes a Fresnel dielectric reflection check at the LSC bottom face, then a mirror reflection check. If the bottom mirror is diffuse, the angle of the photon after reflection is Lambertian random $[159,161]$ :

$$
\begin{aligned}
\phi & =2 \pi \xi_{1} \\
\theta & =\frac{\arccos \left(1-2 \xi_{2}\right)}{2}
\end{aligned}
$$


The photon can bounce multiple times between the LSC bottom face and the bottom mirror.

When computing the probability of reflection from the PV cell surface, the transfer matrix method of computing reflections from a dielectric stack with Fresnel equations is used $[162,163]$. The stack, as detailed in the beginning of this section, consists of the LSC matrix, AB9093 bond layer, $\mathrm{SiN}$ ARC, $\mathrm{SiO}_{2}$ passivation layer, and the $\mathrm{Si} \mathrm{PV}$ cell. The LSC matrix and PV cell layers are treated as having infinite thickness. Reflectivity for light with p- and s- polarizations is calculated and averaged for each angle and wavelength with a resolution of $1^{\circ}$ and $1 \mathrm{~nm}$. Reflectance at $45^{\circ}$ as a function of wavelength is shown in figure 3.6.

\subsection{Baseline Simulation}

Using the realistic "default" values above, a baseline simulation was run to form the basis of comparison for later results which demonstrate the effect of altering a single variable at a time. The full output of the simulation will be shown here as a further introduction to the model; subsequent simulations will show only key results for brevity.

Table 3.1 shows the probability of a photon terminating in a given location. From this matrix and an additional flag (boolean variable) inside of the program to track whether a photon has been self absorbed (SA), SA_flag, the results of table 3.2 can be derived. SA_flag is activated once a photon has been absorbed by a luminophore, emitted, and then absorbed by another luminophore. For this simulation, the LSC output sides left and right are mirrored, and the other two, side $_{1}$ and side $_{2}$, are bonded to a PV cell. The top is open, and the bottom is fitted with a diffuse mirror with an air gap. So, optical efficiency $\eta_{\text {opt }}=\left(\operatorname{side}_{1}\right)+\left(\operatorname{side}_{2}\right)$. The three mirrors $($ left $)+($ right $)+($ bottom $)=($ total mirror loss $)$. QY loss can be separated with the help of SA_flag into two categories: QY loss during the initial absorption event for a given photon $\left(1^{\text {st }} \mathrm{QY}\right)$, or QY loss after at least the second absorption event for that photon, i.e. after being self absorbed (SAQY loss). The matrix and band gap locations 
Table 3.1: Simulated final photon locations as a function of relative QD concentration $N$ for an LSC with "default" values listed above. Each column reports the fraction of photons at each location, and each row sums to one. Here, left and right are mirrors, while side ${ }_{1}$ and side $_{2}$ are PV cells. Photons can also terminate in top plane of the LSC, in the bottom mirror, inside of a $\mathrm{QD}$, or in the matrix.

\begin{tabular}{|l|llllllll|}
\hline$N$ & Left & Right & side $_{1}$ & side $_{2}$ & Top & Bottom & QD & Matrix \\
\hline 0 & 0.0013 & 0.0013 & 0.0408 & 0.0409 & 0.8524 & 0.0330 & 0.0000 & 0.0303 \\
0.25 & 0.0027 & 0.0026 & 0.0811 & 0.0814 & 0.6921 & 0.0294 & 0.0731 & 0.0377 \\
0.5 & 0.0034 & 0.0033 & 0.1052 & 0.1063 & 0.5827 & 0.0268 & 0.1289 & 0.0434 \\
0.75 & 0.0038 & 0.0039 & 0.1221 & 0.1217 & 0.5058 & 0.0241 & 0.1717 & 0.0469 \\
1 & 0.0042 & 0.0041 & 0.1330 & 0.1329 & 0.4466 & 0.0222 & 0.2069 & 0.0502 \\
1.5 & 0.0045 & 0.0045 & 0.1454 & 0.1463 & 0.3680 & 0.0190 & 0.2581 & 0.0543 \\
2 & 0.0048 & 0.0046 & 0.1521 & 0.1522 & 0.3192 & 0.0164 & 0.2940 & 0.0566 \\
2.5 & 0.0048 & 0.0048 & 0.1558 & 0.1553 & 0.2874 & 0.0142 & 0.3200 & 0.0577 \\
3 & 0.0048 & 0.0048 & 0.1568 & 0.1566 & 0.2655 & 0.0128 & 0.3405 & 0.0583 \\
5 & 0.0048 & 0.0046 & 0.1552 & 0.1551 & 0.2259 & 0.0087 & 0.3879 & 0.0578 \\
\hline
\end{tabular}

directly become matrix absorption and sub-band gap losses, respectively. Since the lamp used in this simulation emits no sub-band gap photons, this column is omitted from table 3.1 and table 3.2 .

The location top is comprised of escape cone, unabsorbed radiation, and front surface reflection (FSR) losses. A photon lost from the top is considered an escape cone loss if it has previously absorbed and emitted by a luminophore. If the photon has never been absorbed and emitted within the LSC, the photon must have passed through the LSC, reflected from the bottom mirror, and passed through again before being lost (unabsorbed radiation), or the photon must never have entered the LSC (FSR). FSR is calculated with Fresnel equations to separate these two losses. Escape cone loss can also be categorized as loss during the initial absorption event or those related to self absorption in the same way as QY losses.

Some losses from table 3.2 are plotted in figure 3.7. In the plot, escape cone and QY losses are both a combination of losses during the initial absorption event $\left(1^{\text {st }} \mathrm{L}_{\mathrm{EC}}\right.$ and $\left.1^{\text {st }} \mathrm{QY}\right)$ and secondary self absorption related losses $\left(\mathrm{SAL}_{\mathrm{EC}}\right.$ and $\left.\mathrm{SAQY}\right)$. It is easy to see that there is an optimum luminophore concentration for efficient LSC performance. As the concentration increases, unabsorbed radiation decreases as expected. However, escape cone and QY losses 
Table 3.2: Losses for an LSC as a function of relative QD concentration $N$ with the "default" values listed above. Losses include escape cone losses after self absorption ( $\mathrm{SAL}_{\mathrm{EC}}$ ), escape cone losses after the initial absorption $\left(1^{\text {st }} \mathrm{L}_{\mathrm{EC}}\right)$, unabsorbed radiation (unabs), front surface reflection (FSR), QY loss after self absorption (SAQY), QY loss after the initial absorption $\left(1^{\text {st }} \mathrm{QY}\right)$, matrix absorption (matrix), and mirror absorption (mirror). Each column reports the fraction of photons lost, and each row sums to one when including the optical efficiency $\left(\eta_{\text {opt }}\right)$.

\begin{tabular}{|l|lllllllll|}
\hline$N$ & SAL $_{\mathrm{EC}}$ & $1^{\text {st }} \mathrm{L}_{\mathrm{EC}}$ & unabs & FSR & SAQY & $1^{\text {st }} \mathrm{QY}$ & matrix & mirror & $\eta_{\text {opt }}$ \\
\hline 0 & 0.0000 & 0.0000 & 0.8182 & 0.0341 & 0.0000 & 0.0000 & 0.0303 & 0.0356 & 0.0817 \\
0.25 & 0.0027 & 0.0403 & 0.6149 & 0.0341 & 0.0046 & 0.0685 & 0.0377 & 0.0346 & 0.1625 \\
0.5 & 0.0077 & 0.0654 & 0.4755 & 0.0341 & 0.0130 & 0.1160 & 0.0434 & 0.0335 & 0.2115 \\
0.75 & 0.0133 & 0.0817 & 0.3767 & 0.0341 & 0.0221 & 0.1496 & 0.0469 & 0.0318 & 0.2438 \\
1 & 0.0184 & 0.0924 & 0.3016 & 0.0341 & 0.0312 & 0.1757 & 0.0502 & 0.0306 & 0.2658 \\
1.5 & 0.0276 & 0.1049 & 0.2013 & 0.0341 & 0.0476 & 0.2105 & 0.0543 & 0.0279 & 0.2917 \\
2 & 0.0352 & 0.1110 & 0.1389 & 0.0341 & 0.0612 & 0.2328 & 0.0566 & 0.0258 & 0.3044 \\
2.5 & 0.0419 & 0.1134 & 0.0979 & 0.0341 & 0.0727 & 0.2474 & 0.0577 & 0.0238 & 0.3111 \\
3 & 0.0470 & 0.1143 & 0.0700 & 0.0341 & 0.0825 & 0.2579 & 0.0583 & 0.0224 & 0.3134 \\
5 & 0.0609 & 0.1104 & 0.0205 & 0.0341 & 0.1105 & 0.2773 & 0.0578 & 0.0181 & 0.3104 \\
\hline
\end{tabular}

both rise due to more photons being absorbed and isotropically emitted. Additionally, self absorption losses will rise and then level off as all emitted photons within the self absorption region are lost. Matrix losses increase slowly with concentration because there are more photons traveling through the matrix. Mirror losses decrease with increasing luminophore concentration because photons become more likely to be self absorbed than to propagate long distances and reflect from mirrors. In the special case of $N=0, \eta_{\mathrm{opt}}>0$ because the diffuse back mirror reflects some light into the PV cells.

$\eta_{\text {opt }}$, shown in figure 3.8 , is the fraction of photons reaching a PV cell. The quick rise of $\eta_{\text {opt }}$ with luminophore concentration is understood as a reduction of the unabsorbed radiation loss, while the rounding off and slow decline is primarily from increased self absorption. In reality, this decline happens more quickly than simulated results would imply, as discussed in the next section.

PCE can be calculated from $\eta_{\text {opt }}$ assuming some parameters of the PV cell are known:

$$
\mathrm{PCE}=\eta_{\mathrm{opt}} \cdot \mathrm{EQE} \cdot F F \cdot V_{\mathrm{oc}}
$$


where EQE is the external quantum efficiency of the PV cell at the output wavelength, FF is the fill factor, and $V_{\mathrm{oc}}$ is the open circuit voltage. If $V_{\mathrm{oc}}$ is known for at least one value of photon flux for a given system, it can be estimated for other values using the relation [164]:

$$
V_{\mathrm{oc}}=\frac{k_{\mathrm{B}} T}{e} \ln \left[\frac{I_{\mathrm{sc}}}{I_{0}}+1\right]
$$

where $k_{\mathrm{B}}$ is Boltzmann's constant, $T$ is absolute temperature, $e$ is the elementary charge, $I_{\mathrm{sc}}$ is short circuit current, and $I_{0}$ saturation current density. Since $I_{\mathrm{sc}} \approx \eta_{\mathrm{opt}} \cdot E Q E \cdot($ photon flux $)$, equation 3.12 can be used to scale $V_{\mathrm{oc}}$ for an arbitrary photon flux using $\eta_{\mathrm{opt}}$ in place of $I_{\mathrm{sc}}$ by first solving for $I_{0}$ at a known $V_{\mathrm{oc}}$.

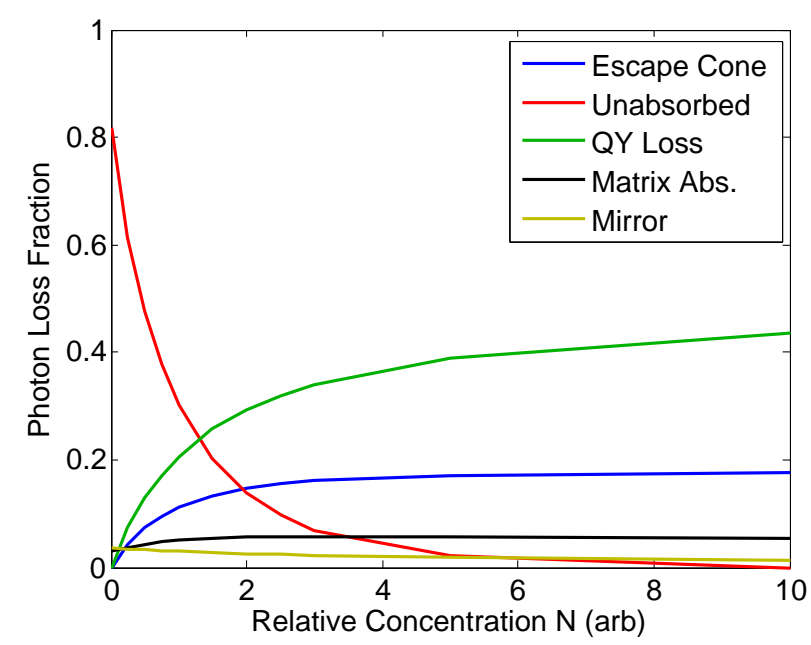

Figure 3.7: Selected simulated LSC losses as a function of relative QD concentration. Unabsorbed radiation loss dominates for low concentration, while escape cone and QY losses dominates at higher concentration.

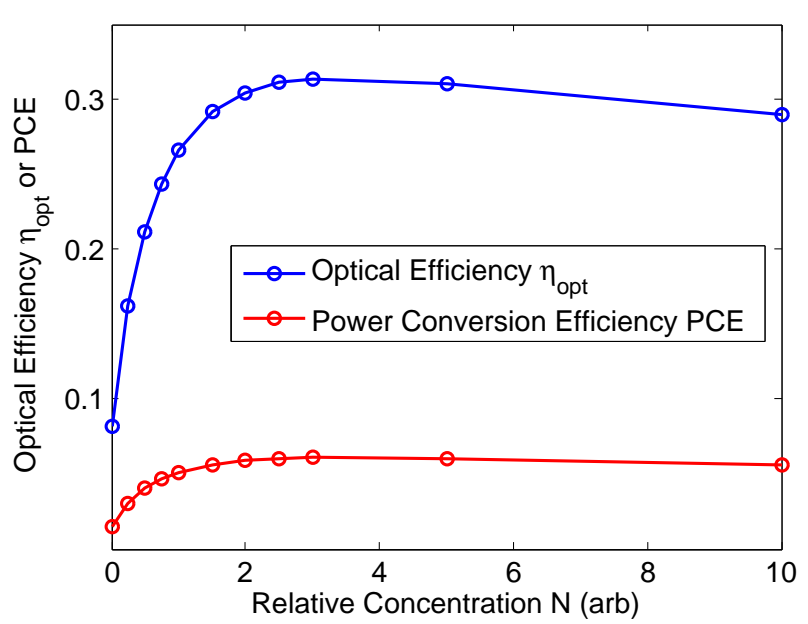

Figure 3.8: $\eta_{\text {opt }}$ and PCE as a function of concentration. $\eta_{\text {opt }}$ quickly rises with concentration as unabsorbed radiation loss decreases, but then levels and falls as escape cone and QY losses rise.

When the LSC is measured with a source that does not emit photons below the band gap of the solar cell, an additional multiplication factor can be applied to approximate the results under solar illumination. While broadband, the OSL1 lamp, for example, does not emit below the $E_{\mathrm{g}}$ of silicon. But, about $36.9 \%$ of solar photons (AM 1.5) are below $E_{\mathrm{g}}$. By multiplying the PCE under lamp illumination by $1-0.369=0.631$, the PCE under solar illumination can be estimated. 
A histogram of the LSC output spectrum can be seen in figure 3.9, along with an overlay of the QD emission spectrum and matrix absorption (both arbitrarily scaled). A red shift from the QD emission spectrum is apparent, a result of self absorption. Some photons outside of the QD emission spectrum also make it to the PV cell, a result of light reflecting from the diffuse bottom mirror directly into the cell without being absorbed and emitted. As the QD concentration increases, the amount of photons not first absorbed before being output by the LSC decreases rapidly, and the red shift increases. Finally, a dip in the output right of the peak can be seen due to the absorption of the matrix.
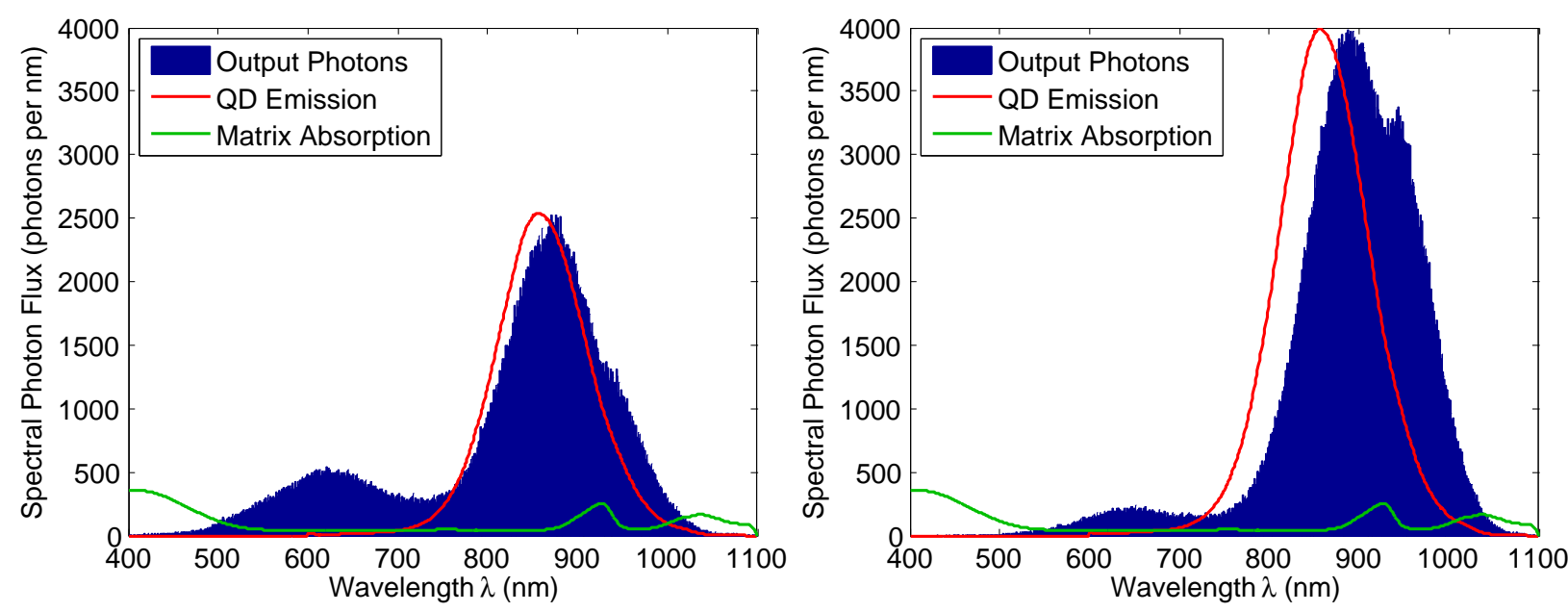

Figure 3.9: Histogram of simulated output wavelengths; (left): a low concentration $(N=0.5)$ and (right): a concentration near maximum $\eta_{\text {opt }}(N=2)$; each bin is $1 \mathrm{~nm}$ in wavelength wide. A red shift of emission can be seen when compared to the original QD emission spectrum, a result of self absorption. Some of the source photons make it to the PV cells by reflecting from the bottom mirror, seen here as photons with $\lambda<\sim 725 \mathrm{~nm}$. A dip in output can be seen, a result of matrix absorption. As $N$ increases, less unabsorbed photons from the source reach the PV cells, and the spectrum is further red shifted. QD emission and matrix absorption are arbitrarily scaled.

An angular histogram of the simulated LSC's photon output angles is shown in figure 3.10. $0^{\circ}$ is in the plane of the LSC and normal to the PV cell. When $N=0$, there can be no guided light: with no luminophores to capture incident light, it will simply pass through the LSC. The only output in this case is light reflecting from the bottom mirror (large spike $>45^{\circ}$ ) or from the bottom mirror and the top face of the LSC (small spike $<-45^{\circ}$ ). As the concentration increases, unabsorbed and thus unguided light decreases and the output is 

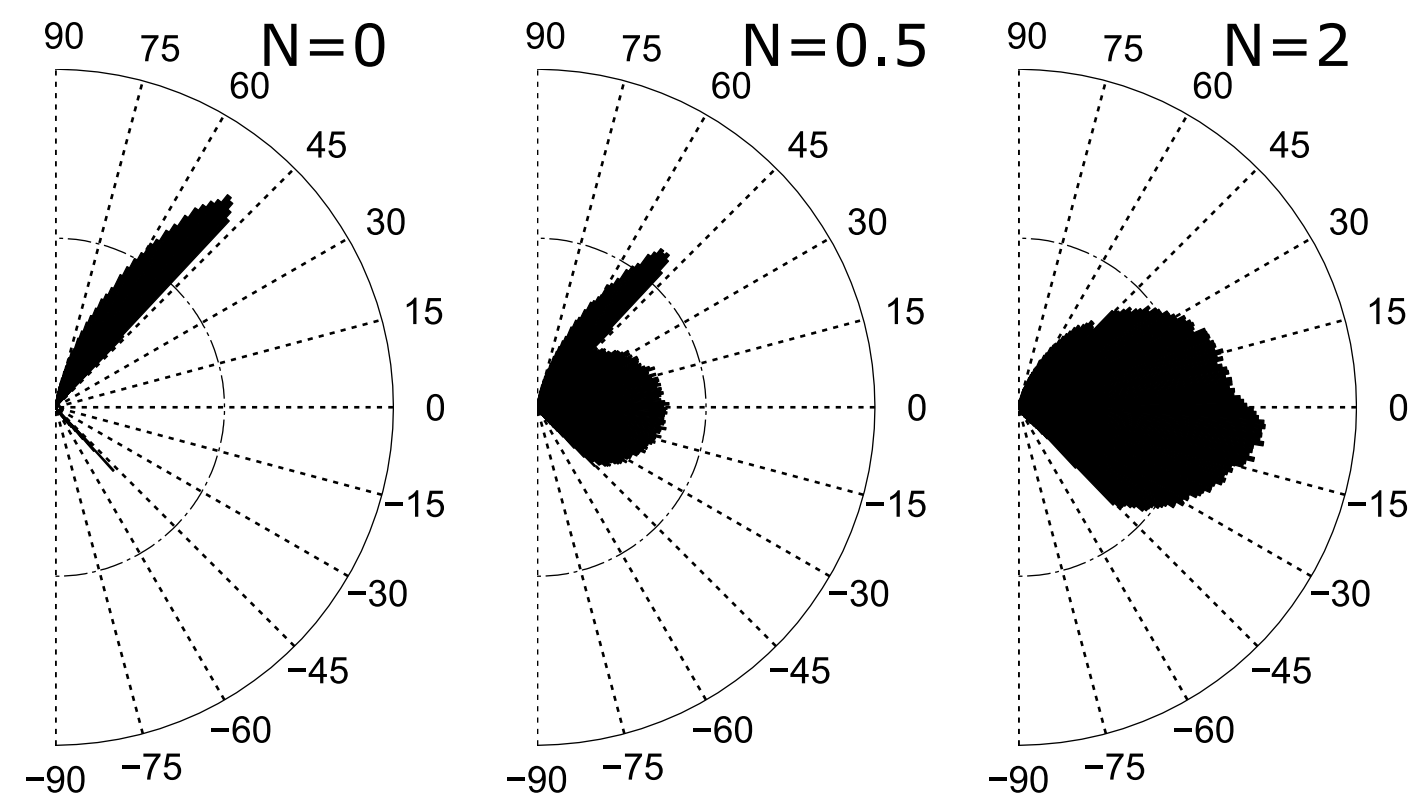

Figure 3.10: Angular histogram of simulated LSC photon output angles at a concentration of $N=0,0.5$, and 2 ; each bin is $1^{\circ}$ wide. $0^{\circ}$ is in the plane of the LSC, normal to the PV cell surface. At $N=0$ there are no guided photons, only those reflected from the bottom mirror (large spike $>45^{\circ}$ ) and reflected from the bottom mirror and the top of the LSC (small spike $<-45^{\circ}$ ). As the concentration increases, more is absorbed and guided near or less than the critical angle for TIR, $\theta_{c}$. The outer circle represents 10,000 photons per bin.

made up primarily of photons absorbed and emitted at least once. This guided emission falls off rapidly outside of the range $-\theta_{\mathrm{c}}$ to $\theta_{\mathrm{c}}$ as the probability of reflection from the dielectric interface falls rapidly.

Finally, the average distance traveled by photons in the LSC can be seen in figure 3.11. The distance traveled by output photons rises quickly from $0.96 \mathrm{~cm}$ at $N=0$ to $2.27 \mathrm{~cm}$ near the concentration of maximum $\eta_{\mathrm{opt}}$, then slowly falls. At $N=0$, most photons are directly from the rear mirror at relatively high angle, so they need not travel very far to get to the PV cell. As $N$ increases slightly, photons are captured and guided, and must travel a bit further on average to reach the PV cell. As $N$ continues to increase, all photons travel a shorter distance before being lost to self absorption related losses. The travel distance of photons is on the same order as the LSC size, $2.5 \times 2.5 \mathrm{~cm}$. 


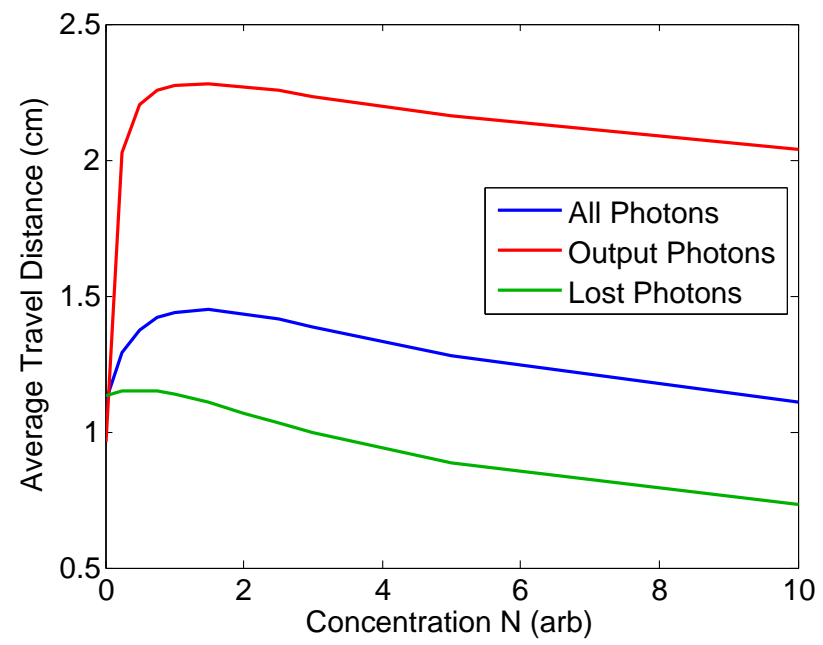

Figure 3.11: The average travel distance of photons inside of the LSC. The distance increases rapidly with $N$ as more photons are absorbed and guided in the LSC instead of reflected directly toward the PV cell from the back mirror. The distance traveled by all photons then decreases as $N$ increases further because self absorption related events decrease the distance photons can travel before being lost.

\subsection{Model Verification and Limitations}

In order to verify the model, the "default" case above was chosen to match the parameters of the highest efficiency LSC discussed in chapter 7. In addition to the many outputs giving reasonable and expected results, the actual device has a PCE of $4.93 \%$, compared to the simulated prediction of $6.07 \%$. The model matches relatively well, but a predicts a bit high. A simulation was also run to compare to experimental values for the current state of the art dye LSC [10]. When exact details for a particular parameter were not available in the literature, reasonable estimations were used. Parameters which differ from the default case listed in section 3.1 are listed here:

- rectangular LSC dimensions of $50 \mathrm{~mm} \times 50 \mathrm{~mm} \times 5 \mathrm{~mm}$,

- matrix material: PMMA

- refractive index $n=1.5$

- absorbance as a function of wavelength, similar to that shown in figure 3.1 
- luminophore: mix of CRS040 (a.k.a. CFS002) yellow [165,166] and Lumogen F Red 305 dye,

$-\mathrm{QY}=0.95$

- absorbance and fluorescence intensity as a function of wavelength, as shown in figure 3.12

- PV cells on all edges of the LSC,

$-V_{\mathrm{oc}}=1 \mathrm{~V}$ (specified)

$-\mathrm{EQE}=0.8$ (estimated)

$-\mathrm{FF}=0.795$ (specified)

- wavelength at the band gap $\lambda_{\mathrm{g}}=850 \mathrm{~nm}$ (estimated); fraction of above band gap photons 0.557 (calculated)

- simulated sunlight with normal incident angle,

- TIR loss per bounce $L_{\mathrm{b}}=0.02$.

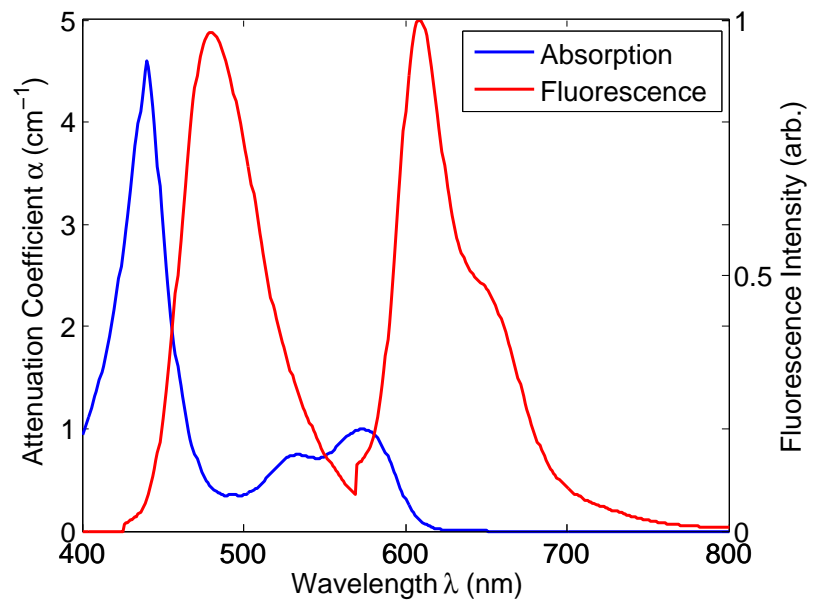

Figure 3.12: The combined spectra of CRS040 and Red 305 dye. The large first absorption peak is a combination of the single absorption peak of CRS040 and one of the three Red 305 absorption peaks. The shorter wavelength single fluorescence peak belongs to CRS040 and the longer wavelength set belongs to Red 305 .

The PCE and $\eta_{\text {opt }}$ for this combined dye system is shown in figure 3.13. Immediately apparent is that $\eta_{\mathrm{opt}}$ increases indefinitely as concentration $N$ goes up. As seen from these results and those for the QD LSC, the model seems to predict too high of a $\eta_{\text {opt }}$ in general, 
but especially for the dye. This monotonic increase in $\eta_{\mathrm{opt}}$ is not expected: self absorption losses (escape cone and QY losses) are expected to eventually overwhelm any gains from lower unabsorbed radiation losses. As seen in figure 3.14, this is not the case. The primary cause of this is the resolution in the input data. The absorption spectrum should have a long low intensity "tail" that continues throughout the fluorescence spectral region. At low concentrations this tail is negligible, but at very high concentrations it will begin to absorb heavily and increase self absorption, countering the gains from the increased solar absorption. The QD input absorption spectra data has a longer measured tail, so the $\eta_{\text {opt }}$ does not monotonically increase.

The increase in $\eta_{\text {opt }}$ begins to round off near $N=2$, implying that the peak should soon follow, perhaps at $N=3$ or 4 . Then the predicted PCE is $7.8 \%$ at $N=4$, close to the measured $7.1 \%$ in the experiment. This further validates the simulation.
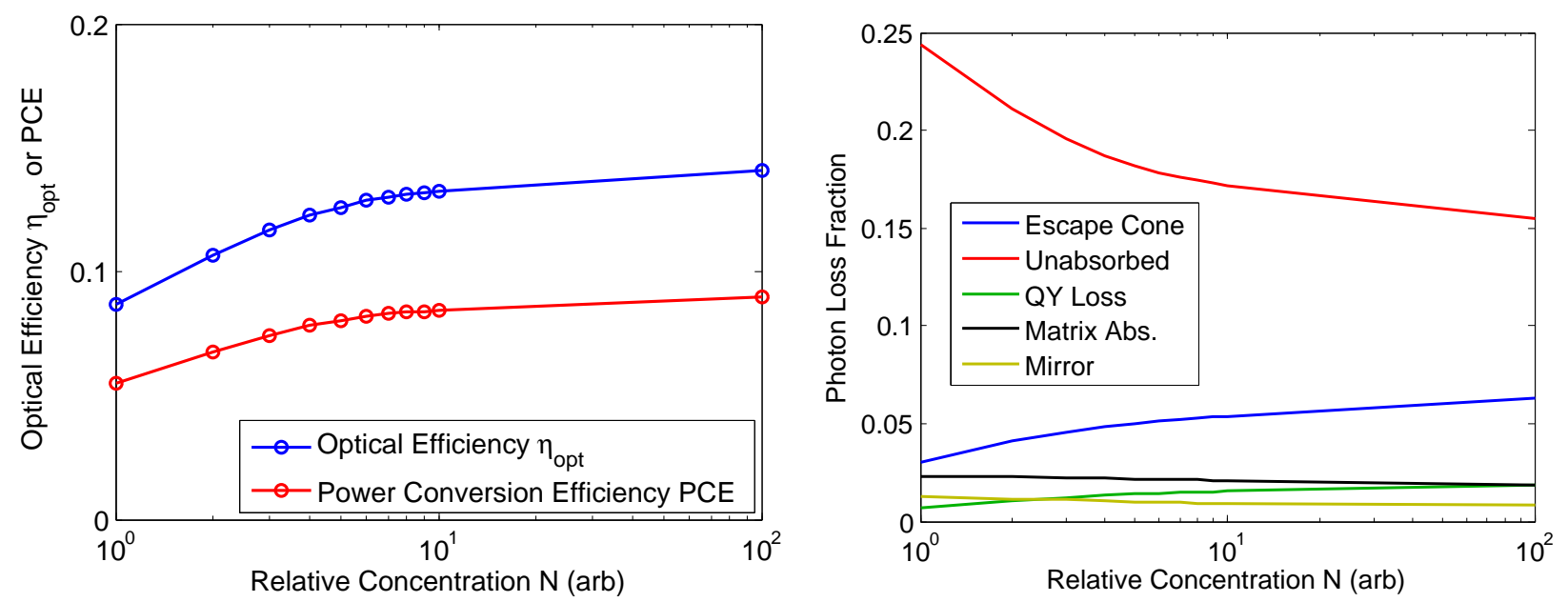

Figure 3.13: $\eta_{\text {opt }}$ and PCE as a function of Figure 3.14: Simulated losses for the comconcentration. $\eta_{\text {opt }}$ quickly rises with concen- bined dye system as a function of relative dye tration as unabsorbed radiation loss decreases. concentration $N$.

It is expected that with further increase in $N$, $\eta_{\text {opt }}$ would level off and fall.

Even accounting for this, the slow drop in $\eta_{\text {opt }}$ with increasing $N$ does not match experiment where a faster drop after the ideal $N$ is observed, although the maximum $\eta_{\text {opt }}$ is predicted well. This implies some other loss mechanism at high concentration not accounted for in the 
model. The largest loss is likely due to scattering. Scattering will increase with luminophore concentration. This is especially true if the luminophore tends to aggregate and clump at higher concentration. Even a change in the local concentration will alter the apparent refractive index of the material and cause scattering. This can easily happen during curing: as the polymer cures, dyes and QDs get expelled from areas that begin to cure first, creating luminophore gradients [167]. The model assumes the attenuation coefficient $\alpha$ to be comprised only of an absorbing component and neglects the scattering component, meaning that an incoming photon will be absorbed and potentially guided instead of scattered and possibly lost.

Other factors can also contribute to experimental results with efficiencies lower than predicted. Imperfect mating to the PV cell, for example, can lower the number of photons able to couple from the LSC to PV cell. Two common ways for this to occur are bubbles/voids in the bonding adhesive and an adhesive fillet, as shown in figure 3.15. The likelihood of Förster Resonance Energy Transfer (FRET) is also increased with increasing luminophore concentration. This non-radiative energy transfer mechanism can cause a decrease in luminescence in a process called concentration quenching [73] and is also not accounted for in the model.

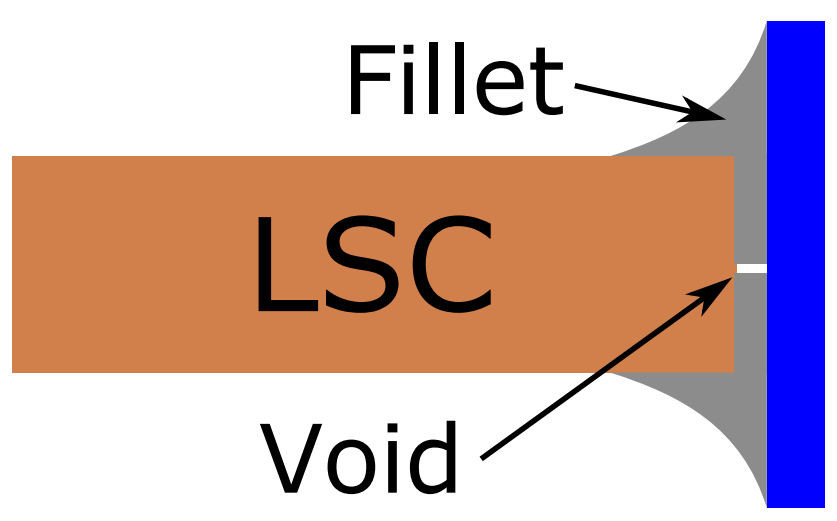

Figure 3.15: Diagram showing fillets and voids that occur when the LSC is bonded to a PV cell. Both flaws will reduce $\eta_{\text {opt }}$.

It should also be noted that the Beer-Lambert Law, on which this model is based, is usually violated at high luminophore concentration, and so should be applied carefully. 
A final limitation of the model is its ability to appropriately predict the output spectral shape for luminophores with more than one emission peak. The output spectrum for the combined dye system is shown in figure 3.16. The spectrum is bimodal, matching the combined fluorescence spectra of the dyes. However, it should be unimodal, matching the fluorescence spectra of only the longer wavelength dye. This is an artifact of the way the model selects an emission wavelength in an absorption event. Though it guarantees the emission photon wavelength is longer than the absorbed photon wavelength, it does so by narrowing the entire emission spectrum. The correct method in a multi-dye system would be to track which dye the photon was absorbed by and only allow emission within that dye's emission spectrum. However, this is a minor difference and does little to change the results of anything other than the predicted output spectral shape.

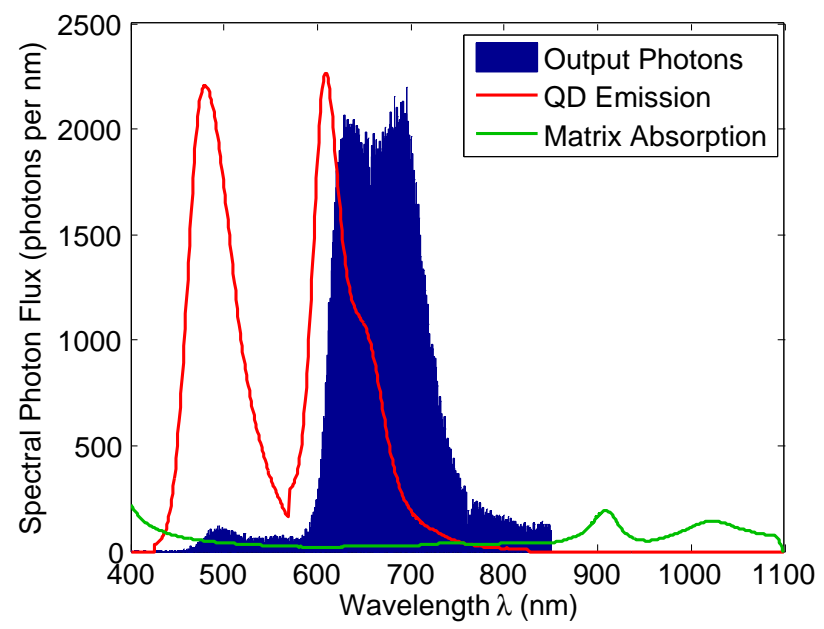

Figure 3.16: Simulated output spectrum for the combined dye LSC system. Though it is improperly predicted to be bimodal, the red shift is still visible. Some of the solar source is also part of the output.

\subsection{Effect of Changing Individual LSC Parameters}

This section will investigate the effects of changing individual parameters of the LSC. With the exception of the variable under test, parameters are set to the default case laid out in section 3.1. 


\section{Quantum Yield}

QY of the luminophore is one of the most important factors to achieving high PCE, as shown in figure 3.17. In this system, each percent increase in QY results in an additional $0.57 \%$ absolute increase in $\eta_{\text {opt }}$. It is interesting to note that for QDs with QY $<0.25$, it is detrimental to use any QDs in the LSC because $\eta_{\text {opt }}$ is reduced below the level achieved with only reflections from the diffuse bottom mirror towards the PV cells.

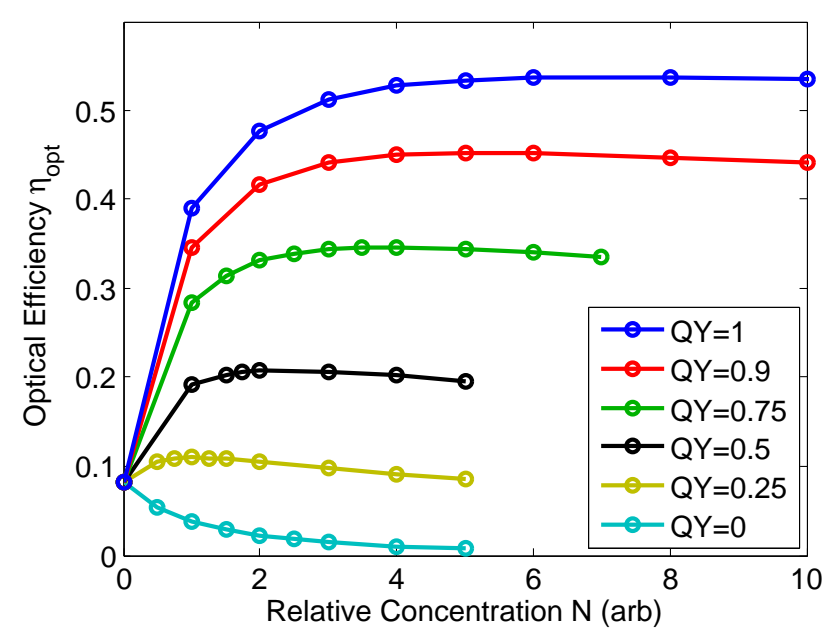

Figure 3.17: Simulated LSC $\eta_{\text {opt }}$ for various QYs as a function of QD concentration $N$.

\section{Bottom Mirror}

For highest $\eta_{\mathrm{opt}}$, a bottom mirror should be used to reflect unabsorbed radiation and escape cone losses back into the LSC. As shown in figure 3.18, ideally this surface should be diffuse so that the reflected photon has a longer path length back through the LSC to increase the chances of being absorbed and guided or directly reach the attached PV cell. This will increase $\eta_{\mathrm{opt}}$. The concentration $N$ at which maximum $\eta_{\mathrm{opt}}$ is achieved is lower with a bottom mirror because photons have an additional pass through the LSC to be absorbed. 


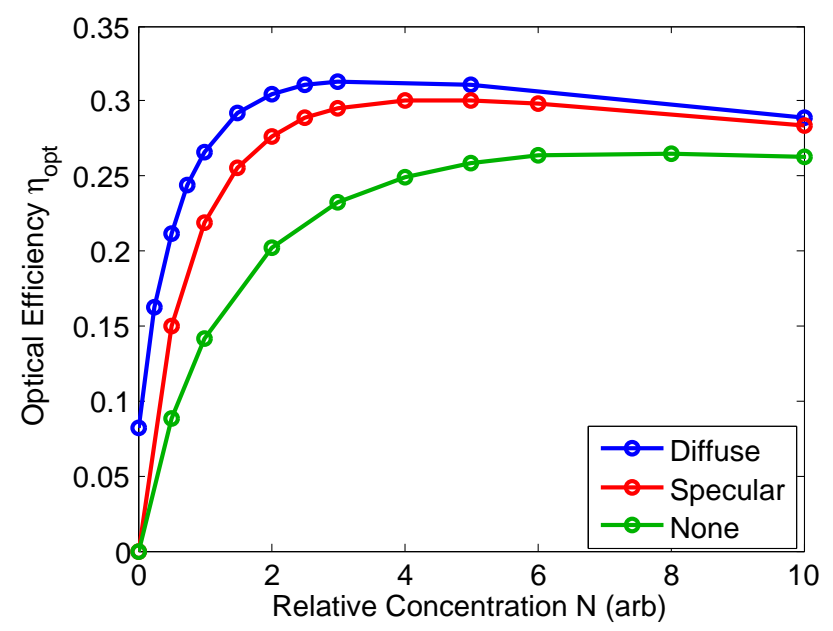

Figure 3.18: Simulated LSC $\eta_{\mathrm{opt}}$ for various bottom mirror types as a function of QD concentration $N$.

\section{Anti-Reflection Coating}

PV cells are generally coated with an anti-reflection coating (ARC) to maximize their efficiency for the solar spectrum. However, the spectral distribution from an LSC is far different from the solar distribution. As shown in figure 3.19, the reflectivity minimum can be pushed to longer wavelengths to more closely match the QD emission spectrum by increasing the thickness of the ARC. However, the gain in $\eta_{\mathrm{opt}}$ is very modest, as shown in figure 3.20, suggesting that the losses due to reflection from the PV cell face are low.

\section{Matrix Absorption}

For a matrix with a refractive index of $n=1.5$ at the $\mathrm{D}_{1}$ line, the various matrix absorption values shown in figure 3.1 were simulated. In addition to AB9093 and P(LMA-co-EGDMA) matrices, a perfectly clear matrix with no attenuation was simulated, as well as matrices with $1 \%$ and $5 \%$ attenuation per cm traveled. As shown in figure 3.21, AB9093 marginally outperforms P(LMA-co-EGDMA), primarily because of its lower absorption in the near IR. There is an $11.9 \%$ relative change in $\eta_{\text {opt }}$ from $\mathrm{P}(\mathrm{LMA}-\mathrm{co}-\mathrm{EGDMA})$ to a clear matrix, and a 9.5\% relative change from AB9093 to a clear matrix. 

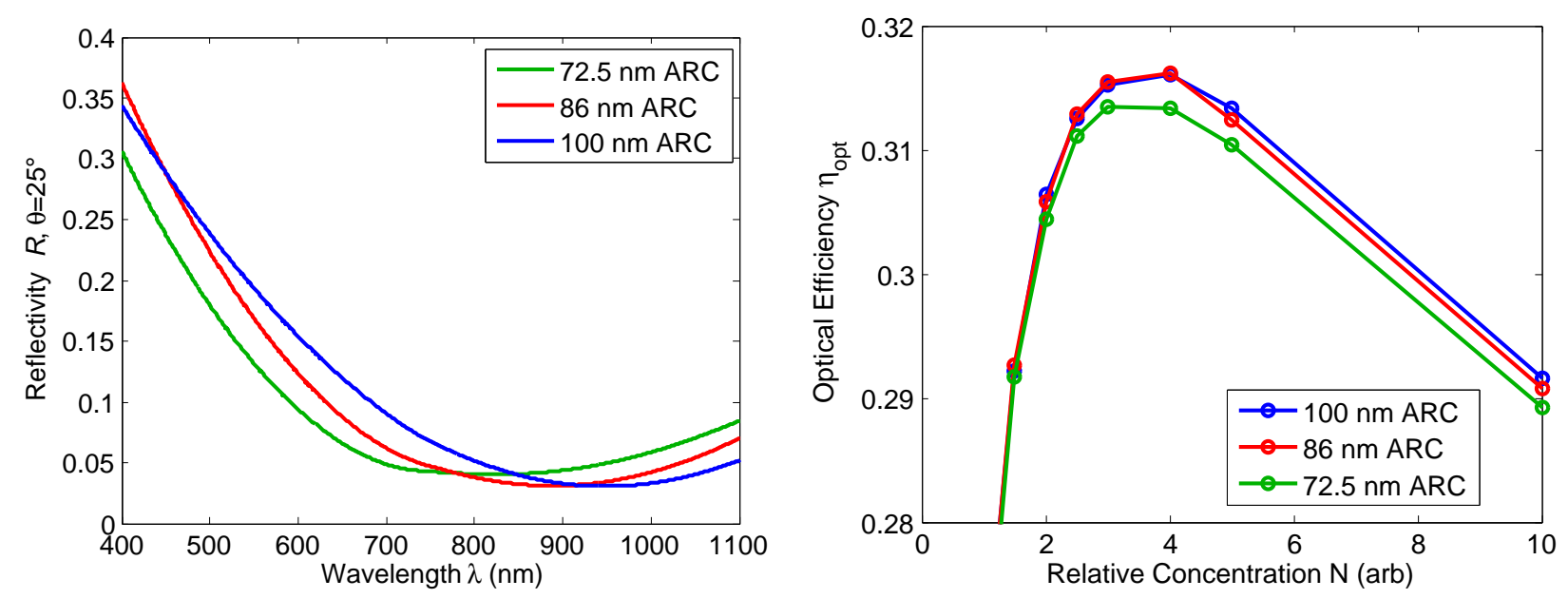

Figure 3.19: Reflectivity of the PV cell inter- Figure 3.20: Simulated LSC $\eta_{\text {opt }}$ for various face with various $\mathrm{ARC}$ thicknesses at $\theta=25^{\circ}$. PV cell ARC thicknesses as a function of QD The reflection minimum can be shifted to concentration $N$. longer wavelengths by increasing the thickness of the ARC.

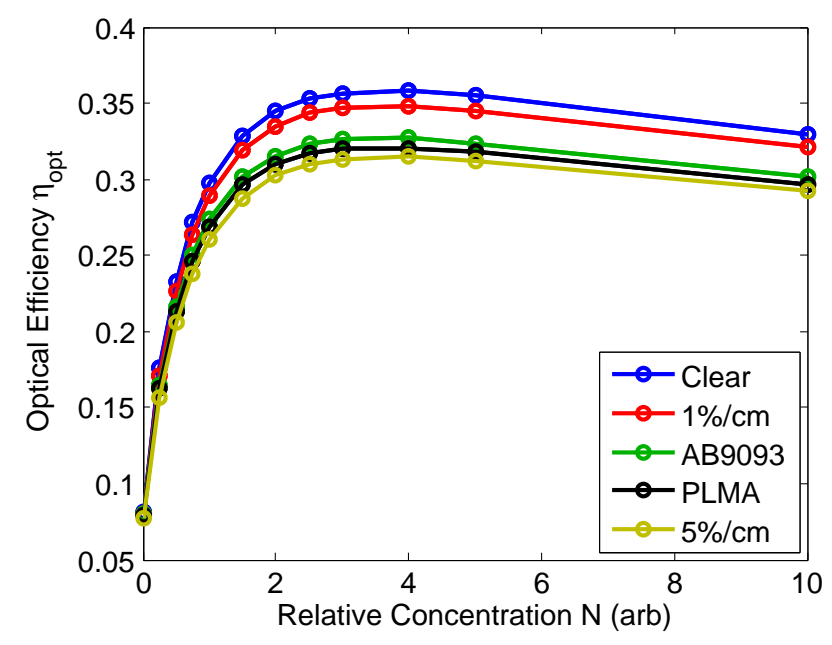

Figure 3.21: Simulated LSC $\eta_{\text {opt }}$ for various matrix absorption values as a function of QD concentration $N$.

\section{Mirror Reflectivity}

High reflectivity mirrors are important when fabricating an LSC, as shown in figure 3.22. When the reflectivity of all mirrors is decreased from $R=1$ to $R=0.97$, there is a relative loss in overall $\eta_{\mathrm{opt}}$ of $2.5 \%$. Assuming the side mirrors are appropriately bonded with no voids or defects, this is about the highest reflectivity which could be expected. 


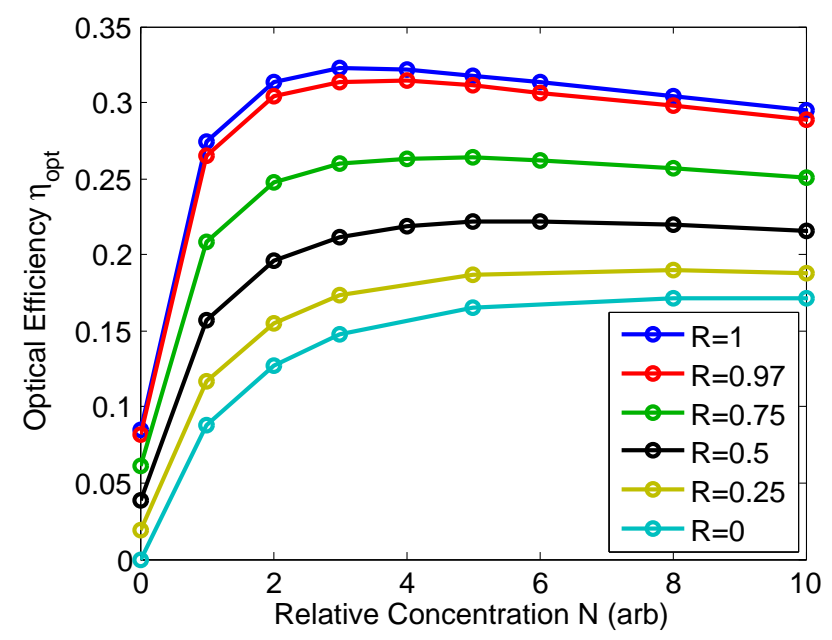

Figure 3.22: Simulated LSC $\eta_{\mathrm{opt}}$ for various mirror reflectivities as a function of QD concentration $N$.

\section{Index of Refraction}

In section 2.4.1, the ideal matrix refractive index was calculated to be $n=1.99$. While simulation matches this result, it also reveals a more complicated story, as shown in figure 3.23. This is because the simple formula does not account for a diffuse back mirror or self absorption. At lower QD concentrations, $\eta_{\text {opt }}$ is higher at $n=1.5$. At higher $N, \eta_{\text {opt }}$ is higher at $n=2$. This is because at high concentration, there are more self absorption events which present an opportunity for the photon to be lost from the escape cone. A greater $n$ is then favorable because it will trap more photons at the expense of slightly higher FSR loss.

\section{TIR Loss}

TIR loss is a relatively minor loss, as shown in figure 3.24 , simply because photons do not bounce many times before they are output or lost to another loss mechanism in an LSC of this size. It would become a larger loss if the LSC were scaled up, as it is a constant loss per distance traveled by photons of all wavelengths. For this LSC system, a slightly dirty surface resulting in 3\% loss per bounce would drop $\eta_{\mathrm{opt}} 4.1 \%$, relatively. 


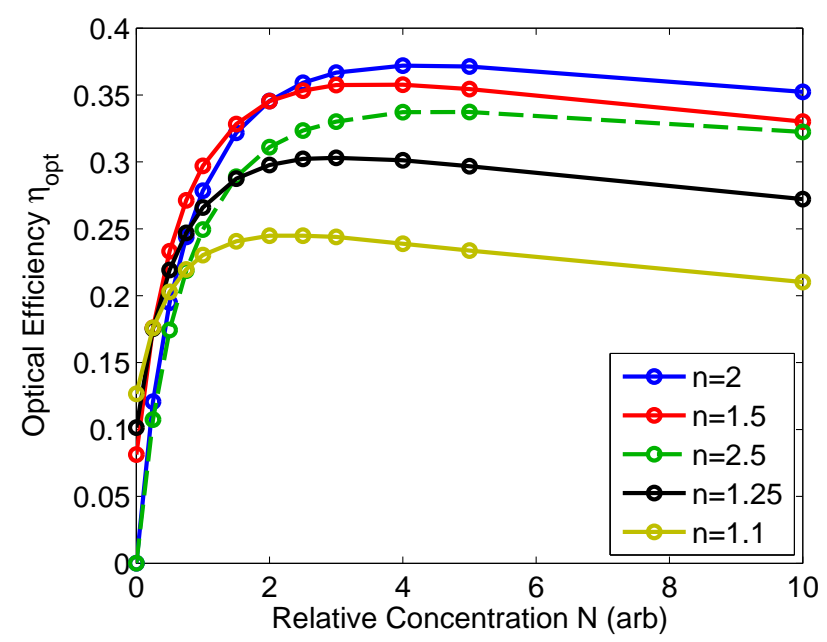

Figure 3.23: Simulated LSC $\eta_{\mathrm{opt}}$ for various matrix refractive indices as a function of QD concentration $N$. Solid lines represent increasing $\eta_{\text {opt }}$ with increasing $n$, while the dotted line represents a decreasing $\eta_{\text {opt }}$ with increasing $n$. Simulated with no matrix absorption losses.

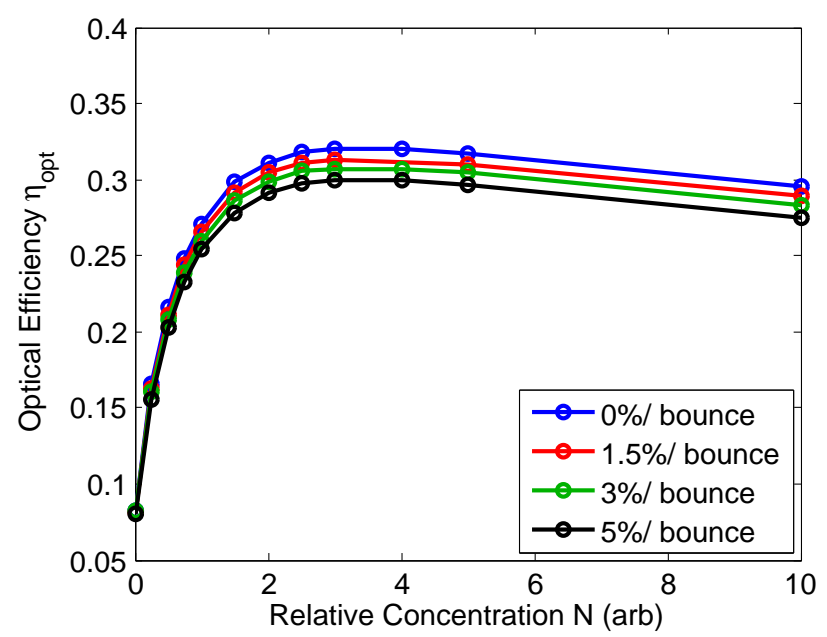

Figure 3.24: Simulated LSC $\eta_{\text {opt }}$ for various TIR loss fraction per bounce as a function of QD concentration $N$.

\section{Spectra Shift}

The effect of shifting the QD absorption and emission spectra was investigated under simulated solar illumination. As seen in figure 3.25, the PCE of the system is increased when the spectra are red-shifted closer to the band gap of silicon. More of the solar spectra can be absorbed at a given $N$, increasing PCE by $7.5 \%$ relatively with a $100 \mathrm{~nm}$ red shift as compared to the un-shifted spectra. 
Another option is to blue shift the spectra enough to take advantage of GaAs PV cells with vastly superior $V_{\text {oc }}$. At low concentrations this option is predicted to outperform the silicon PV cell system, but it's PCE quickly falls as the output spectrum begins to red shift past the band gap of the GaAs PV cell. Blue shifting the spectra further would be very difficult as it would require making the QD smaller, and the QDs used in this work are already near the minimum physical size limit.

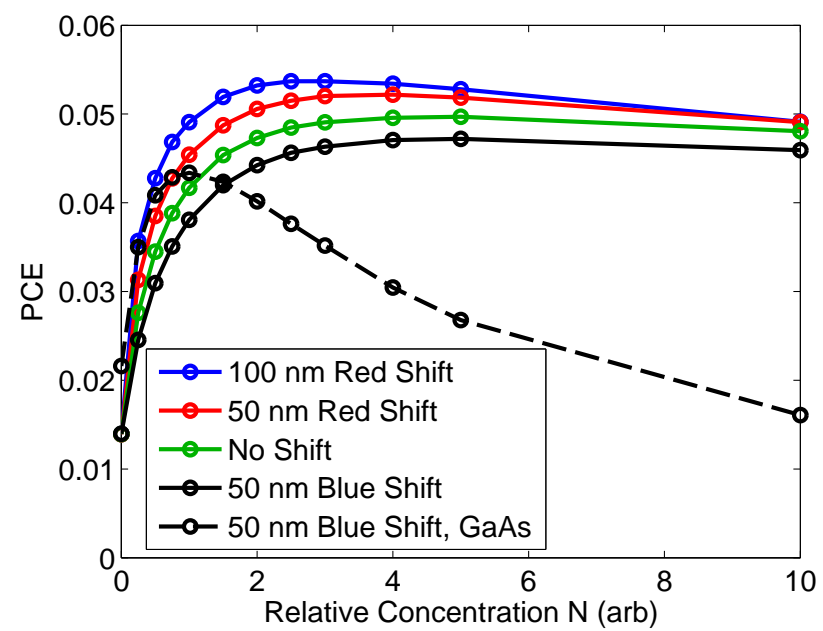

Figure 3.25: Simulated LSC PCE with various luminophore spectra as a function of QD concentration $N$. The dotted line is PCE when mated with a GaAs PV cell instead of a $\mathrm{Si}$ PV cell.

\subsection{Ideal Case}

In order to investigate an "ideal" LSC, a simulation was run with best case parameters for the LSC. The solar cell parameters used are idealized but achievable. The parameters that differ from the default case in section 3.1 are listed here:

- matrix material: no absorption or scattering, $n=1.65$,

- ideal luminophore with complete absorption from $\lambda=300 \mathrm{~nm}$ to $1049 \mathrm{~nm}$, all emission at $1050 \mathrm{~nm}$ (no self absorption), QY $=1$,

- mirror $R=1$ with diffuse bottom mirror, 
- PV cells on opposite edges of the LSC with $V_{\text {oc }}=0.75 \mathrm{~V}, \mathrm{EQE}=0.9, F F=0.8$, and optimized $100 \mathrm{~nm}$ thick ARC,

- simulated solar source at normal incidence,

- TIR loss per bounce $L_{\mathrm{b}}=0$.

The losses in this system are shown in figure 3.26. Photons below the PV cell $E_{\mathrm{g}}$ account for the largest drop in $\eta_{\mathrm{opt}}, 36.9 \%$. Unabsorbed radiation loss drops quickly to zero because of the ideal nature of the luminophore, while the escape cone loss levels off. It does not grow further with concentration because there is no self absorption: all escape cone losses are from the first absorption event.

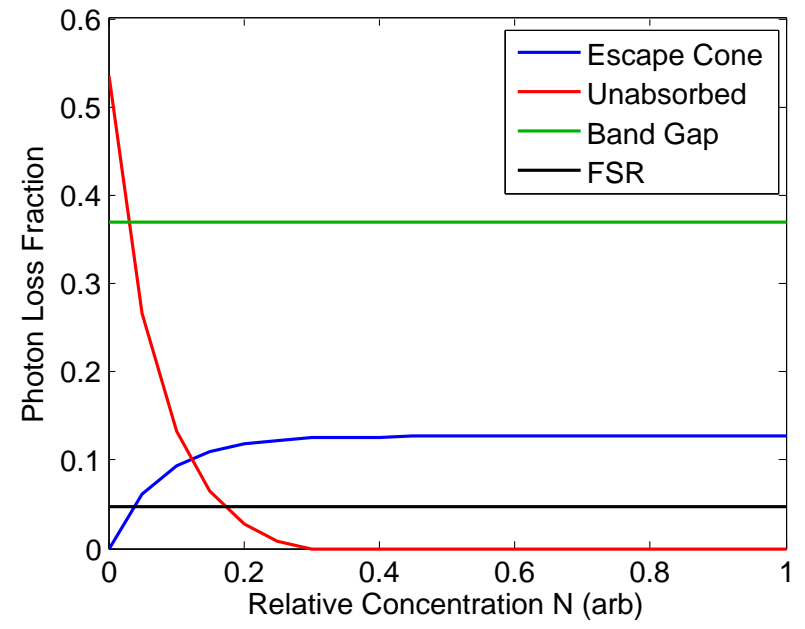

Figure 3.26: Simulated losses in an ideal LSC system. With high enough concentration, unabsorbed radiation losses go to zero and only escape cone, FSR, and band gap losses remain.

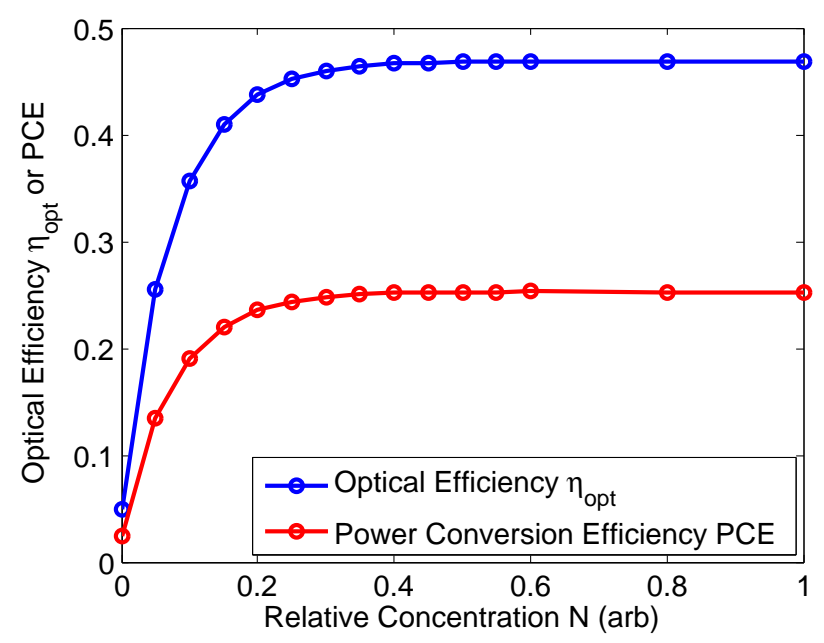

Figure 3.27: Simulated LSC $\eta_{\mathrm{opt}}$ and PCE in an ideal LSC system. These results match those theoretically derived from considering only FSR and escape cone losses after accounting for band gap losses.

The maximum $\eta_{\text {opt }}$ predicted for this system is $46.8 \%$, as shown in figure 3.27. If illuminated with a source with no below band photons, $\eta_{\text {opt }}$ becomes $74.2 \%$. The loss, $25.8 \%$, is then only due to escape cone and FSR. This nearly exactly matches the $26.5 \%$ loss predicted by equations 2.18 and 2.24 (plotted in figure 2.14), thus lending credence to the model with theoretical predictions as well. The extra $0.7 \%$ is from photons which reflect from the rear mirror directly to the PV cell. 


\subsection{Conclusion}

A Monte Carlo ray tracing model was constructed to evaluate the performance of LSCs. The model is robust, able to calculate results for a great number of input conditions. A number of outputs are available to the user beyond the predicted $\eta_{\mathrm{opt}}$, including where in the LSC photons terminated, a breakdown of losses, the LSC output spectrum, photon output angles, and photon average travel distance.

The model was verified against experimental results and observations, literature, and theory. While a great number of loss mechanisms are accounted for by the model, it does neglect the effects of scattering, inter-luminophore effects such as FRET and aggregation, the violation of the Beer-Lambert law at high concentration, and fabrication defects. Thus, the model tends to predict higher than experimental values, especially at a high concentration of high QY luminophores. Additionally, the user must be mindful of input spectra "tails," especially when simulating at concentrations far above the measured concentration.

The model was then used to predict the effect of changing many individual parameters of the LSC to isolate their impact on the system as a whole. Using these results as a guide, an ideal LSC was simulated. The predicted $\eta_{\text {opt }}$ value very closely matched the theoretical predictions of loss due to escape cone and FSR. 


\section{Chapter 4: Experiment}

\subsection{QD Synthesis}

PbSe QDs were synthesized by collaborators at the University of Rochester, Department of Chemistry, and used as delivered in a hexane or toluene solution. The synthesis process is based on a process described by Evans et al. [168] and is described in-depth by Waldron et al. [87].

Briefly, di-i-butylphosphine, selenium shot, and anhydrous toluene are combined to form the first precursor solution. The second precursor solution is created by combining $\mathrm{PbO}$, oleic acid, and decane. The first precursor solution is then swiftly injected into the second solution. The solution instantly turns dark brown, indicative of PbSe QD formation. QD size is controlled by the length of time the solution is left at temperature. After the desired time, about two minutes, an aliquot is pulled and thermally quenched with cold ethanol. This is then centrifuged to separate the supernatant from the QDs, which are then re-dispersed in hexane or toluene. Anhydrous solvents are preferred throughout the process.

The resultant QDs have a core diameter of approximately $2.5 \mathrm{~nm}$. The $\mathrm{Pb}: \mathrm{Se}$ ratio is not stoichiometric because the surface is lead rich $[169,170]$. The dots are capped with oleic acid derived electrically insulating ligands about $2.5 \mathrm{~nm}$ long. QDs used in initial characterization studies had a QY of about $50 \%$ to $60 \%$. Improved synthesis techniques, such as carefully limiting oxygen exposure, led to higher QY (> 70\%) for later studies including LSC fabrication and characterization. 


\subsection{Matrix Preparation and QD Incorporation}

Many matrix material candidates were identified and tested for suitability based on how well they approximate the ideal matrix discussed in section 1.1.3. QDs were delivered and stored in solution. Unless otherwise noted, the QDs were dried under a flow of dry nitrogen to remove all solvent immediately before incorporation into the matrix material.

\subsubsection{Sol-gels}

Titania and zirconia sol-gels were prepared as by Jasieniak et al. [171]. Isopropanol and acetylacetone were mixed under a nitrogen atmosphere with titanium isopropoxide or zirconium isopropoxide, respectively, to form the sol-gel solution. This solution was mixed with the QDs and spun unto a glass substrate at $3500 \mathrm{rpm}$ to form a thin film. This film was then measured as is, or first annealed in an oven at up to $100^{\circ} \mathrm{C}$ for five minutes.

\subsubsection{Cast PMMA}

PMMA powder (Lucite Elvacite ${ }^{\circledR}$ grade 2009) was dissolved in a solvent compatible with the QDs such as chloroform or toluene to form a very viscous solution. QDs dispersed in the same solvent were mixed in and the resultant solution was degassed and cast onto a glass plate to dry. The drying time was slowed by placing the sample in a partially sealed chamber.

The resultant film was about $65 \mu \mathrm{m}$ thick. This film was peeled from the glass and cut into squares $\sim 2.5 \mathrm{~cm}$ on a side, coated with a two part, optically clear epoxy (EpoxySet brand EB-107LP-2), and stacked to form a thicker waveguide, $\sim 0.5 \mathrm{~cm}$ thick.

\subsubsection{Cured PMMA}

Liquid MMA monomer was mixed with a powdered thermal or UV activated radical initiator, azobisisobutyronitrile or bis(2,4,6-trimethylbenzoyl)-phenyl phosphine oxide, respectively. 
The initiator was varied from $0.1 \%$ to $1 \%$ by weight. This solution was mixed with QDs and cured. Thermally cured samples were poured into a Teflon mold sealed between two aluminum plates and placed in a temperature controlled water bath to cure. Temperatures ranging from $60^{\circ} \mathrm{C}$ to $90^{\circ} \mathrm{C}$ were employed for different samples. UV cured samples were cured as described in section 4.2.7.

\subsubsection{Two part epoxies}

Smooth-On brand Crystal Clear 202 [172], EpoxySet brand EB-107LP-2 [173], and Epo-Kwik embediment resin were used as directed. QDs were mixed with Part A first, then the resulting solution was mixed with Part B and vacuum degassed. The opposite mixing order with Part B first was also tried for each. In each case, the solution was left to cure in a square silicone cup mold, about $2.5 \mathrm{~cm}$ square and $0.5 \mathrm{~cm}$ to $1 \mathrm{~cm}$ thick at room temperature under ambient atmosphere.

\subsubsection{AB9093 Epoxy}

Angstrom Bond brand AB9093 one part, UV curing, 100\% solids epoxy was used as purchased from Fiber Optic Center, Inc. As described in Waldron et al. [87], AB9093 and QDs are mixed in a sonic bath under a nitrogen atmosphere, vacuum degassed, and UV cured as described in section 4.2.7. AB9093 bonds strongly with glass, so the glass sheets used in the mold (figure 4.1) become part of the sample. A maximum QD concentration of $\sim 100 \mu \mathrm{M}$ can be incorporated without aggregation [87].

\subsubsection{P(LMA-co-EGDMA)}

$\mathrm{P}$ (LMA-co-EGDMA) is a copolymer of lauryl methacrylate (LMA) and ethylene glycol dimethacrylate (EGDMA), with the LMA providing compatibility with the QDs and excellent optical properties, and the EGDMA acting as a cross linker to produce a rigid matrix. PLMA 
by itself is gelatinous; higher percentages of EGDMA can be used in the copolymer depending on the rigidity desired, usually up to $30 \%$ by weight.

LMA-co-EGDMA solution was prepared as described by Waldron et al. [174] in a process similar to that described by Bomm et al. [29]. LMA (96\% with 500 ppm MEHQ inhibitor) and EGDMA (98\% with 90-110 ppm MEHQ inhibitor) were sourced from Sigma-Aldrich and used as received. A stock solution of UV initiator (bis(2,4,6-trimethylbenzoyl)-phenyl phosphine oxide, 97\%, Sigma-Aldrich) was formed by dissolving the powder in acetone for easier later measurement by weight: mg-type quantities are required, so measuring a dilute initiator solution was more accurate.

The solution was prepared by mixing $0.1 \%$ UV initiator (after evaporation of acetone), $80 \%$ LMA, and 20\% EGDMA by weight in ambient atmosphere. Under a nitrogen atmosphere, this was then poured on the QDs and mixed by swirling; no sonication is required for homogenization. A QD concentration of at least $100 \mu \mathrm{M}$ can be achieved without aggregation, but an upper limit has not been established [87].

The mixture was UV cured as described in section 4.2.7. P(LMA-co-EGDMA) does not bond with the glass used in the mold (figure 4.1), so the glass was either discarded or subsequently re-attached with AB9093.

\subsubsection{UV Curing}

The UV curing process proceeds as described by Waldron et al. [87]. The mold is constructed from a U-shaped piece of Teflon sandwiched between two sheets of $0.15 \mathrm{~mm}$ thick glass to form a cup, as shown in figure 4.1. Binder clips hold the glass in place. After curing, the Teflon is removed and can be re-used.

Curing takes place with one $15 \mathrm{~W}$ Philips Master Actinic 18 inch length fluorescent bulb on each side of the mold in the absence of oxygen under a nitrogen atmosphere. This bulb emits primarily in the UV region at $368 \mathrm{~nm}$, with other lower energy smaller emission peaks across the visible spectrum. Curing takes a minute or less for clear samples and a few minutes 


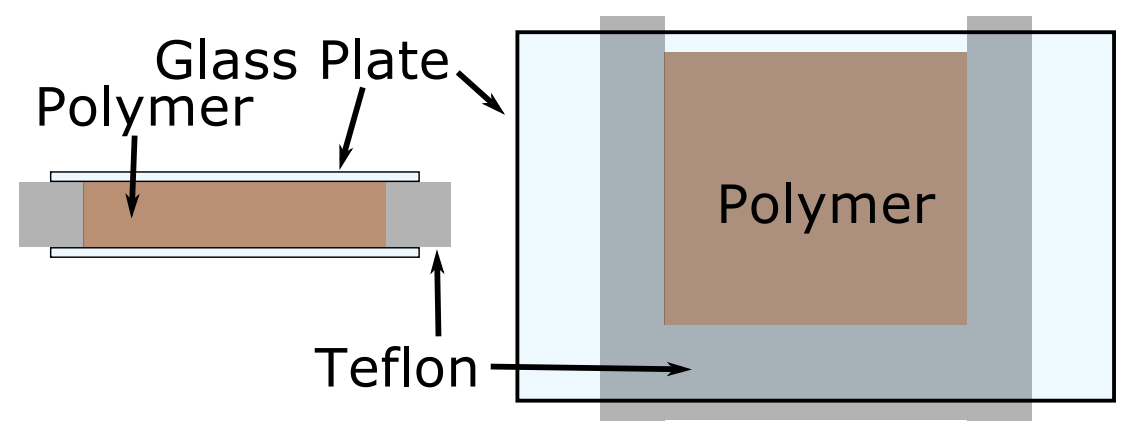

Figure 4.1: Mold for UV cured polymer castings. Left: top view; Right: side view. Gray: Teflon; Light blue: glass plates; Brown: polymer.

to an hour for QD nanocomposites, depending on sample thickness and QD concentration. After curing, the sample edges are ground to size and polished with a Buehler polishing machine.

\subsection{Optical Characterization}

Optical absorption measurements were carried out on a Beckman DU-650 absorption spectrophotometer at between $0.2 \mathrm{~nm}$ and $0.5 \mathrm{~nm}$ wavelength resolution and $\leq 1.8 \mathrm{~nm}$ spectral bandwidth. Measurements of samples containing QDs were referenced to identical samples without QDs - either a clear polymer sample or quartz cuvette with appropriate solvent — such that the absorption shown is of the QDs only, not a combination of QDs and matrix. Clear sample measurements were referenced to air via a blank measurement.

Steady state fluorescence was measured with a StellarNet EPP2000CXR-SR-200 spectrometer at $0.5 \mathrm{~nm}$ resolution, as shown in figure 4.2. A $532 \mathrm{~nm}$ laser was used as an excitation source, and a long-pass absorbing filter (optical density 11) was used to block the excitation wavelength. Time-resolved fluorescence measurements were performed with a PicoHarp 300 and an $850 \mathrm{~nm}$ long-pass filter and a resolution of 128 ps per channel. Lifetime data was fit with MATLAB.

Scattering and QY measurements were performed with a Labsphere RTC-060-SF integrating sphere with a Spectraflect ${ }^{\circledR}$ barium sulfate coating, using the methods described in 


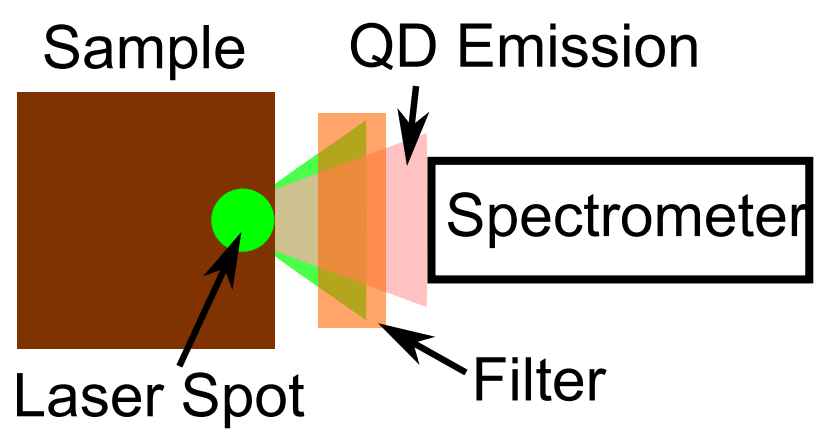

Figure 4.2: Setup for measuring QD nanocomposite fluorescence spectrum. The laser source is kept close the the edge to limit the effects of self absorption on the measured output spectrum.

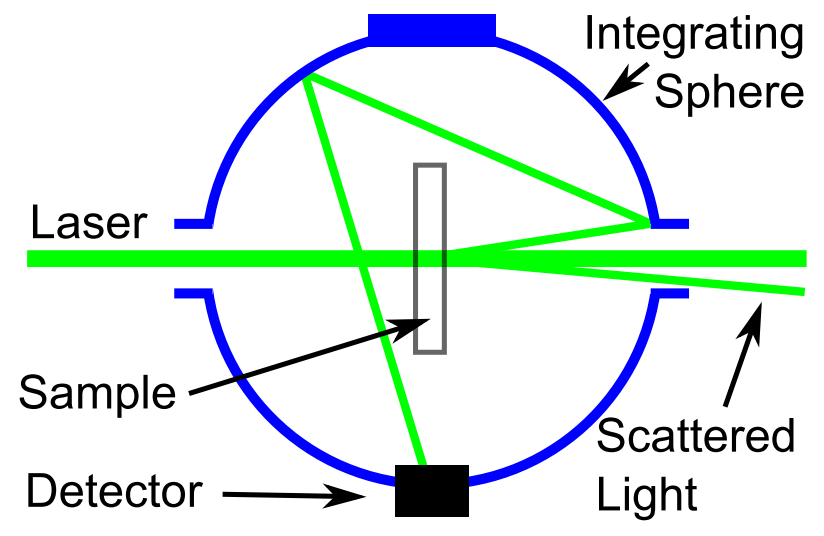

Figure 4.3: Setup for measuring scattered light with an integrating sphere. All light scattered at an angle $>3^{\circ}$ is captured.

Waldron et al. [87]. For scattering measurements, as shown in figure 4.3, laser light is input via a port and through the sample with the FSR returning through the same port. Light scattered at an angle $<3^{\circ}$ is allowed to pass out of the sphere through another port, while light scattered at an angle $>3^{\circ}$ is captured measured by a power meter. $532 \mathrm{~nm}$ doubled Nd-YAG, 632.8 nm HeNe, and 1064 nm Nd-YAG lasers were used as sources.

QY measurements were performed using a $532 \mathrm{~nm}$ laser source to allow it to be easily blocked with an optical filter without affecting the fluorescence measurement. Power collected in the sphere was measured with and without a long-pass filter to block the source wavelength from the power meter while input power was measured separately. The number of photons absorbed and emitted by the sample, and thus the QY, could then be calculated after correction for self absorption as described by Ahn et al. [175].

\subsection{Morphology Characterization by SEM \& TEM}

Analysis of the AB9093 nanocomposite via TEM, SEM, and EDS was attempted. However, the nanocomposite was too soft for the microtome to properly cut a thin sample. A JEOL 
1230 TEM was thus unable to image the sample. SEM and EDS analysis was carried out on a FEI Quanta 650 SEM.

\subsection{Temperature Control Setup and Samples}

An Alpha-Omega Instruments Series 800 PID temperature controller was used in conjunction with a US Sensor PPG101A1 $100 \Omega \pm 0.06 \%$ platinum resistance temperature detector (RTD) and a Thorlabs TEC1.4-6 Peltier-type thermoelectric heater/cooler (TEC) to control the sample temperature within $\pm 0.1^{\circ} \mathrm{C}$ between $0^{\circ} \mathrm{C}$ and $80^{\circ} \mathrm{C}$. The TEC has a $5.5 \mathrm{~mm}$ diameter center hole. All interfaces were coated with Arctic Silver Inc. Céramique thermal compound.

For QD in toluene measurements, a glass cuvette (4 mL capacity, $1 \mathrm{~cm}$ path length) with a Teflon stopper was used with a custom machined aluminum heating sleeve to evenly distribute heat from the TEC, as shown in the left of figure 4.4. The large thermal mass limited the temperature range studied from $15^{\circ} \mathrm{C}$ to $65^{\circ} \mathrm{C}$.

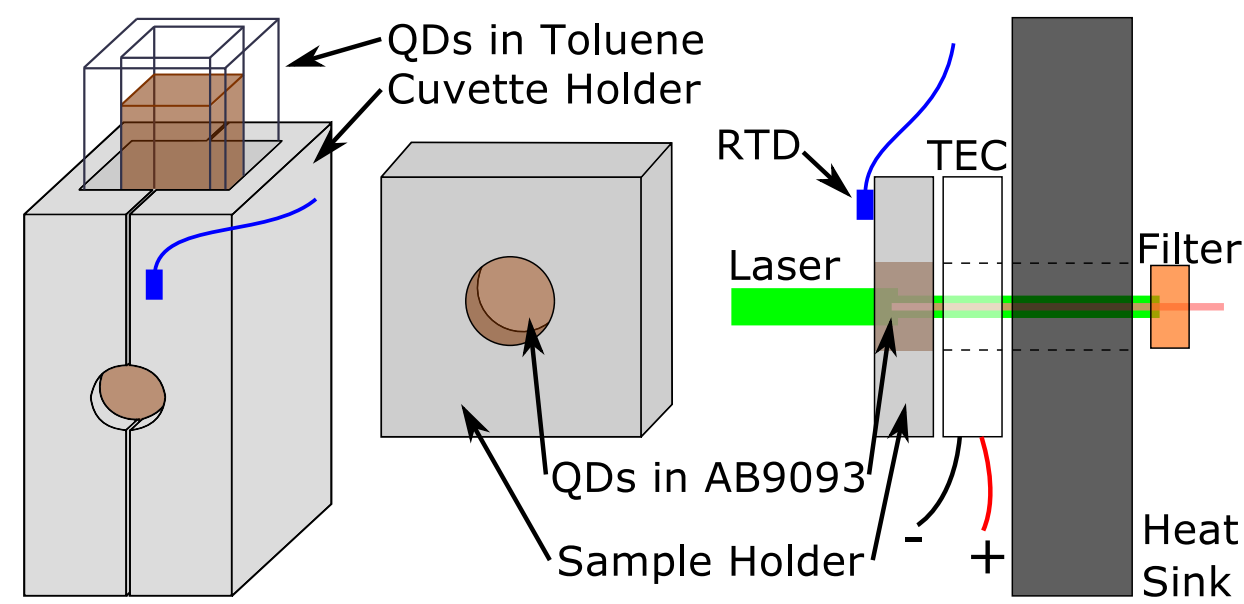

Figure 4.4: Experimental setup. Left: Aluminum cuvette warmer and cuvette full of QD solution. Center: Aluminum sample holder and polymer nanocomposite. Right (from left to right): Holder and sample with RTD temperature probe (blue), TEC, heat sink, and long-pass filter, with green laser passing through, exciting near-IR output.

QDs in AB9093, prepared and cured as in section 4.2.7, were cast directly into a custom machined aluminum holder, shown in the center of figure 4.4 . The aluminum is $\sim 15 \mathrm{~mm}$ tall and wide to match the TEC face dimensions. Two thicknesses of aluminum plate were 
used, $3.3 \mathrm{~mm}$ for fluorescence measurements and $6.1 \mathrm{~mm}$ for absorption measurements. A removable glass bottom plate and a $0.15 \mathrm{~mm}$ thick glass cover slip top plate were used to form the casting well. In both cases, the RTD temperature sensor was directly contacted to the aluminum with thermal compound on the face opposite of the TEC.

Dye samples were prepared with BASF Lumogen ${ }^{\circledR}$ F Red 305 (Kremer Pigments, Inc.) in toluene (Sigma-Aldrich, $99.9 \%$ ) via serial dilution to $0.0025 \%$ by weight and transfered to a $1 \mathrm{~cm}$ path length glass cuvette for measurement. For dye fluorescence measurements, the optical filter was omitted. Instead, the sample was illuminated at an angle such that the laser did not pass entirely through to the rear through-hole.

Optical measurements of QDs and dye in toluene were corrected for the thermal volumetric expansion of toluene, $1100 \mathrm{ppm} /{ }^{\circ} \mathrm{C}[176]$. Because of the increased volume, the luminophore concentration decreases with increasing temperature. Measurements of QDs in AB9093 did not need correction because the thermal expansion coefficient is much lower $\left(60 \mathrm{ppm} /{ }^{\circ} \mathrm{C}[148]\right)$ and the expansion would simply result in a longer path length allowing for identical absorption.

\subsection{LSC PV System Fabrication}

Complete LSC PV systems were created by bonding mirror film and PV cells to LSC samples fabricated as in section 4.2. First, SunPower Corp. monocrystalline Si PV cells, $22 \%$ efficiency (manufacturer specification), were prepared for use. A section of cell was cut away from the wafer after bonding to a small piece of glass to prevent snapping, then select areas of Si were broken away with the aid of a stereoscopic microscope to reach the contacts running along the cell backplane (both positive and negative contacts run along the back of the cell). These contacts were then soldered to a small length of wire and the entire assembly was bonded to an acrylic sheet for mechanical stability. AB9093 epoxy was used to bond the PV cell to the polished LSC emitting face using a UV diode to spot-cure (QPhotonics, LLC model UVTOP355, $365 \mathrm{~nm}$ emission center, $0.4 \mathrm{~mW}$ optical power). 
$3 \mathrm{M}^{\mathrm{TM}}$ Vikuiti Enhanced Specular Reflector (ESR) film was used on the other two opposing LSC edge faces (American Polarizers, Inc.), attached with $3 \mathrm{M}^{\mathrm{TM}}$ Optically Clear Adhesive 8142KCL film (Tekra). The ESR film, $65 \mu \mathrm{m}$ thick polyester, is specified with reflectance $R>98 \%$ and the adhesive film, $2 \mu \mathrm{m}$ thick polyester with index $n=1.47$, is specified at $>99 \%$ transmission in the visible and near-IR spectrum. For measurement, the LSC PV system was placed as close as possible over a diffuse reflector (Spectraflect ${ }^{\circledR}$ barium sulfate coating, Labsphere, Inc.) while maintaining an air gap.

\subsection{Solar Simulation Setup}
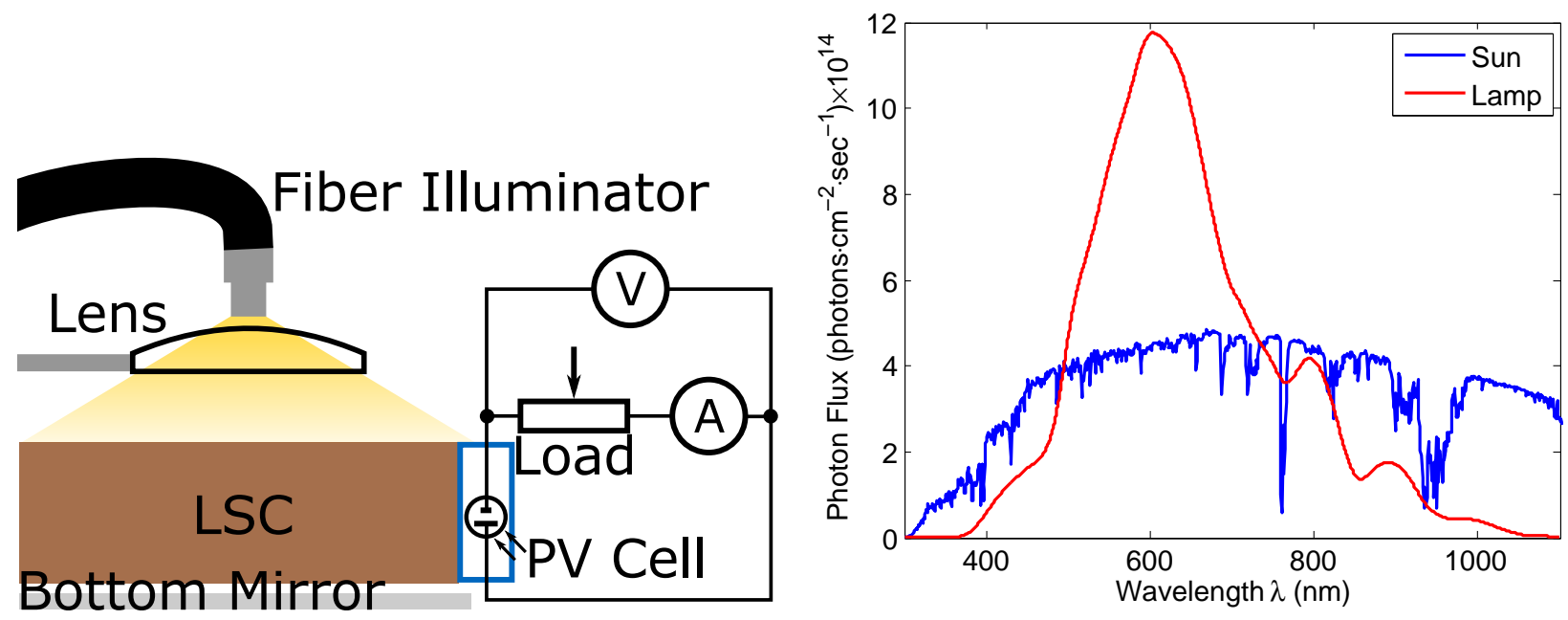

Figure 4.5: Setup for measuring the I-V Figure 4.6: Broadband halogen lamp speccurve of the LSC PV system with diffuse bottom reflector using broadband emission from a halogen fiber illuminator. A lens is used to trum compared to sunlight at the Earth's surface [6]. The photon flux of the lamp has been matched to the solar photon flux below provide more even illumination of the sample. $1100 \mathrm{~nm}$ wavelength. An ammeter and volt meter are used to measure the cell characteristics while varying the load on the cell with a potentiometer.

The setup for measuring the I-V characteristics of the LSC PV system is shown in figure 4.5. A Thorlabs OSL1 fiber illuminator with a $150 \mathrm{~W}$ halogen lamp was used as the light source. A planoconvex lens with focal length $f=25 \mathrm{~mm}$ was used to provide more even illumination across the LSC surface since the fiber output was very divergent. The 
distance between the sample, lens, and fiber tip was adjusted so that the average optical power incident on the LSC surface was $87.3 \frac{\mathrm{mW}}{\mathrm{cm}^{2}}$. As shown in figure 4.6, this represents the lamp optical power required to match the solar photon flux at wavelengths lower than the band gap of the Si PV cell $(1100 \mathrm{~nm})$. Then the photon flux of both the lamp and sunlight with $\lambda \leq 1100 \mathrm{~nm}$ is $\sim 2.75 \times 10^{17}$ photons $/ \mathrm{cm}^{2}$ (representing $81.1 \frac{\mathrm{mW}}{\mathrm{cm}^{2}}$ of the $100 \frac{\mathrm{mW}}{\mathrm{cm}^{2}}$ contained in the entire solar spectrum at Earth's surface). This ensures the LSC will receive equal usable photon flux as if it had been measured under the ASTM standard air mass 1.5 solar spectrum [6] as wavelengths greater than $1100 \mathrm{~nm}$ cannot be absorbed by either the QDs in the LSC or by the attached PV cell.

Optical power was measured with a Melles Griot 13PEM001 thermopile broadband power meter. A ten turn, $100 \Omega, 2 \mathrm{~W}$ potentiometer and a digital multimeter ammeter (HewlettPackard 3468A) was connected to a single PV cell in series as the load for a minimum resistance of $\sim 0.5 \Omega$. A digital multimeter (Fluke $8842 \mathrm{~A}$ ) was connected in parallel across the potentiometer and ammeter load to measure the voltage drop. The potentiometer is then varied to generate an $\mathrm{I}-\mathrm{V}$ curve. 


\section{Chapter 5: QD Nanocomposite}

\section{Characterization}

The optical properties of PbSe QDs in AB9093, P(LMA-co-EGDMA), hexane, and toluene were characterized. Both the fluorescence and absorption across the visible and near-IR spectrum were examined. Additionally, the polymer matrices were examined without QDs to ensure the optical requirements for an LSC matrix material are met.

The other matrix material candidates described in section 4.2 were not included for further study. Sol-gels caused the QDs to aggregate and flocculate out of solution, and best optical properties were not reached until after a high temperature anneal which damaged the QDs. Another difficulty with this material system is forming a matrix with the desired thickness: sol-gels are typically cast as thin films. However, if these difficulties could be overcome, it would be an interesting LSC matrix material to study due to its lack of near-IR absorption and overall clarity (an example transmission curve as a function of wavelength of a similar system is given by Franc et al. [177]).

Cast PMMA suffered from high levels of optical defects and QD damage from oxygen ingress during the long curing process. QDs in both UV and thermally cured PMMA also suffered from a loss of fluorescent output, perhaps as a consequence of the free radicals released during the cure and/or elevated temperatures. In all of the two part epoxies tested, QDs were either insoluble or completely destroyed, characterized by a near complete loss of both optical absorption and fluorescence. Some of these matrix materials have been used successfully with other QDs in the past [178], but proved incompatible with these QDs. This highlights the need to study each different material system. 


\subsection{Matrix Characterization}

As noted in section 1.1.3, the matrix should be both transparent and non-scattering. The optical attenuation properties of AB9093, P(LMA-co-EGDMA), and commercial acrylic glass are shown in figure 5.1.

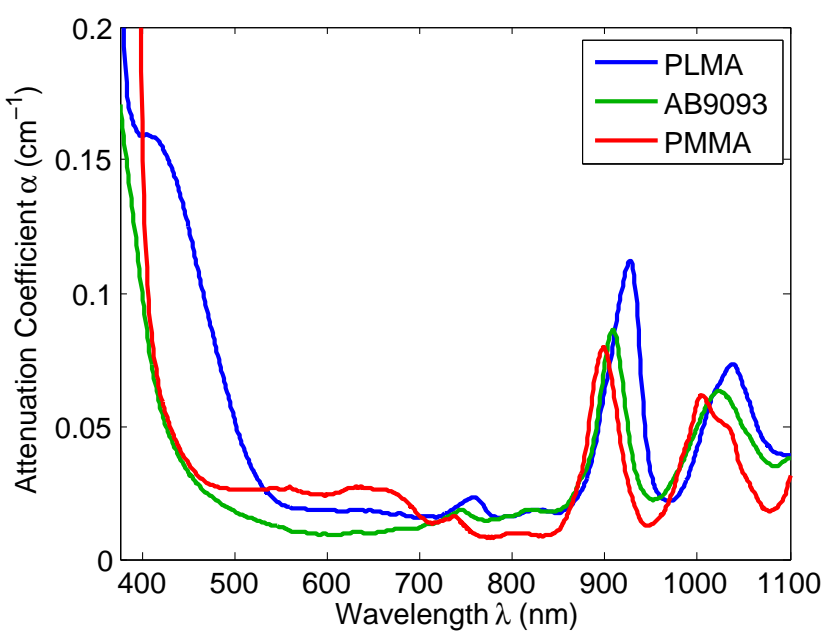

Figure 5.1: Attenuation coefficient as a function of wavelength for various cured polymers including AB9093, P(LMA-co-EGDMA), and commercial PMMA for comparison. Both AB9093 and P(LMA-co-EGDMA) are suitable for use as a matrix in terms of low optical attenuation.

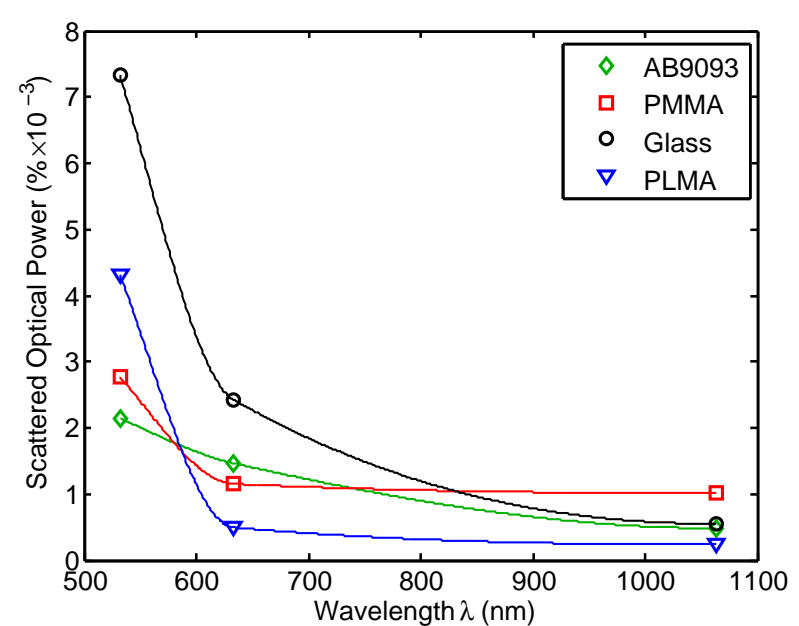

Figure 5.2: Optical power scattered at an angle of $>3^{\circ}$ through AB9093, PMMA, glass, and $\mathrm{P}(\mathrm{LMA}-\mathrm{co}-\mathrm{EGDMA})$ at three wavelengths. Lines shown are piecewise polynomial interpolations as a guide for the eye.

Both AB9093 and P(LMA-co-EGDMA) are very transparent over the region of interest. All polymers begin to be highly absorbing below about $\lambda=400 \mathrm{~nm}$, although P(LMA-co-EGDMA) does exhibit increased absorption starting at $\lambda=500 \mathrm{~nm}$, likely the result of a UV absorption tail from the polymerization inhibitor used to transport and store the constituent monomers [132]. The commercial PMMA has a higher transmission cut-on wavelength than expected for pure PMMA because of a UV absorber additive. Given the similarity of the AB9093 transmission cut-on wavelength to the commercial PMMA, it is likely the AB9093 contains a similar additive. P(LMA-co-EGDMA) also suffers from a 
slightly lower transmission across the entire spectrum of interest compared to AB9093, and all polymers measured absorb minimally in the near-IR region.

The variation of light scattering with wavelength through the same clear samples is shown in figure 5.2. Scattering decreases with wavelength as expected, and all samples demonstrate $<0.01 \%$ optical power scattered at an angle of $>3^{\circ}$.

\subsection{Optical Absorption Study}

QD absorption spectra will change depending on the surrounding matrix. Especially significant is the wavelength position and intensity of the first excitonic "1-s" peak. Figure 5.3 shows data on absorption verses wavelength for QDs in hexane, toluene, AB9093, and P(LMA-co-EGDMA), as well as bulk PbSe for comparison. Using the 1-s peak approximation, the band gap of the QDs in each matrix is $1.472 \mathrm{eV}, 1.496 \mathrm{eV}, 1.366 \mathrm{eV}$, and $1.509 \mathrm{eV}$, respectively; the bulk PbSe band gap is $0.27 \mathrm{eV}$. All curves are normalized to $75 \%$ absorption at $532 \mathrm{~nm}$ for ease of comparison. The absorption coefficient $\alpha$ for bulk PbSe is calculated from the extinction coefficient $\kappa$ given in the SOPRA database at a given wavelength $\lambda$ with $\alpha=4 \pi \kappa / \lambda[179]$. The refractive index of each material at the $\mathrm{D}_{1}$ Fraunhofer line is: hexane, $n=1.375$ [91]; toluene, $n=1.496$ [180]; AB9093, $n=1.56$ [148]; and P(LMA-co-EGDMA), $n=1.45$ (see section 3.1).

The QD 1-s peak red shifts $65 \mathrm{~nm}$ from $842.5 \mathrm{~nm}$ to $907.5 \mathrm{~nm}$ when moved from hexane to AB9093, $27 \mathrm{~nm}$ further than the red shift of the fluorescent peak (figure 5.4). This means the Stokes shift decreases, increasing self absorption and potentially lowering LSC performance. This red shift can be a result of QD aggregation: the wave functions of nearby QDs overlap and the QDs begin to act more like bulk material due to a lowering of confinement. To ensure this was not the case, SEM and EDS analysis was performed. Though little else could be gleaned given the resolution and sensitivity limitations, no aggregates of QDs could be found. Instead, it is likely that much of this shift is due to a change in the size distribution of the 


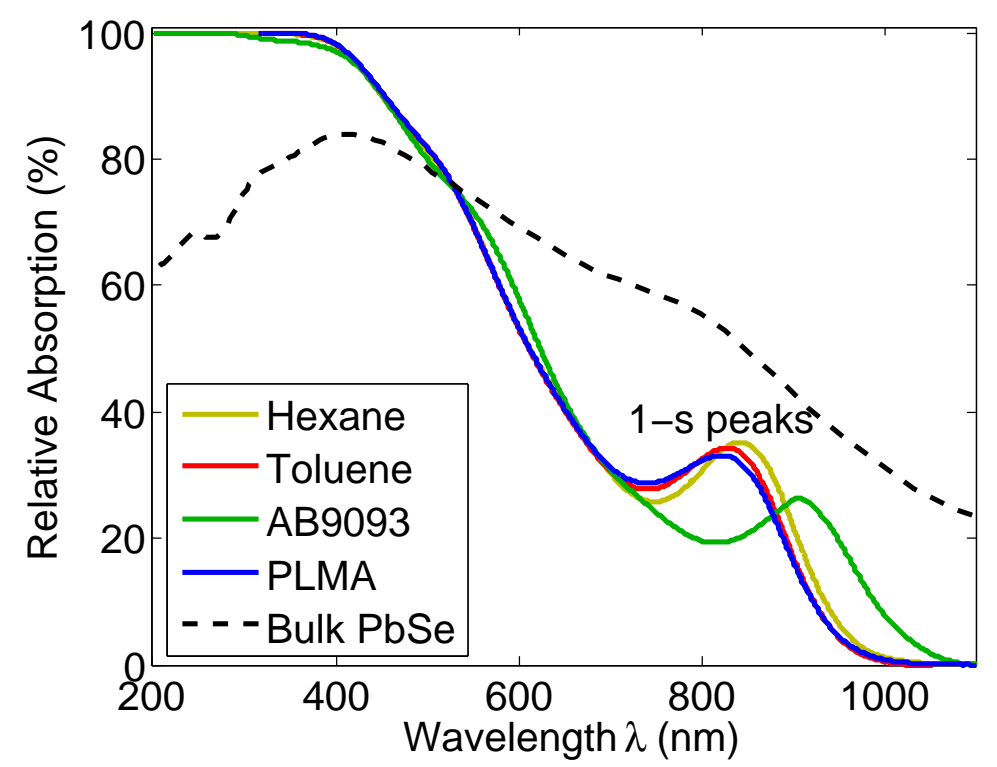

Figure 5.3: QD optical absorption in hexane, toluene, AB9093, and P(LMA-co-EGDMA). Absorption of bulk PbSe is also shown for comparison. Notice the dependence of the 1-s absorption peak position on the matrix material.

emitting population. With their larger surface area to volume ratio, the smaller QDs are more likely to be damaged and taken out of the emitting population, leaving a relatively higher number of larger QDs. These larger QDs will have a lower energy band gap and absorb and emit at longer wavelengths.

The 1-s peak in toluene and P(LMA-co-EGDMA) blue shifts $13.5 \mathrm{~nm}$ to $829 \mathrm{~nm}$ and $21 \mathrm{~nm}$ to $821.5 \mathrm{~nm}$, respectively, implying increased LSC performance from a wider Stokes shift resulting in lower self absorption.

\subsection{QD Fluorescence Study}

The QY and fluorescence wavelength both depend not only on the QD but also its matrix. Some of this change is based on the dielectric constant of the matrix, but the majority of the change is due to QD damage. Protective ligands can be lost during incorporation into the matrix, increasing the number of surface trap states available as well as the probability of non-radiative decay, leading to fluorescence red shifting and a reduction of QY. This is 
a result of the increased photoluminescent decay time associated with an exciton being in a trap state. The exciton looses energy while in the trap state, resulting in a lower energy fluorescent photon or no emission at all.

This effect can also be seen if the QD concentration is high or if the QDs are not well dispersed, as excitons will begin to tunnel between QDs. SEM/EDS analysis of the AB9093 and QD nanocomposite was unable to find any evidence of large (>10 nm) clusters of QDs, implying good dispersion.

Oxidation damage can also occur and is accelerated by light exposure, as QDs are more easily oxidized in an excited state. QY, and thus photoluminescent intensity, is lowered and in some cases the fluorescence can blue shift as the QDs become physically smaller.

As shown in figure 5.4, the emission of the QDs in AB9093 is red shifted by about $38 \mathrm{~nm}$ with respect to the original hexane solution, from a wavelength of $934 \mathrm{~nm}$ to $972 \mathrm{~nm}$. The QY falls by about half, from $55 \%$ to $26 \%$. On the other hand, there is basically no change in fluorescence wavelength or QY when the QDs are moved to toluene or P(LMA-co-EGDMA). However, QDs in P(LMA-co-EGDMA) tend to quickly photo-degrade, reaching $10 \%$ or less of their original output power with an hour or two of exposure to a few mWs of $532 \mathrm{~nm}$ laser light. When the light is blocked for a time and turned back on, much of the original fluorescence intensity returns, though how much depends on exposure time and intensity.

Dielectric constant plays a minor roll in the shift in emission spectra. As an example, the change in $E_{\mathrm{g}}$ of the QD due to a polarization energy change when moving from hexane to AB9093 can be calculated via equation 2.9, repeated here:

$$
E_{\mathrm{pol}}=\frac{e^{2}(\epsilon-1)}{2 \pi \mathrm{R}^{2} \epsilon_{\mathrm{QD}} \epsilon_{0}} \int_{r=0}^{\mathrm{R}} \sin ^{2}\left(\frac{\pi r}{\mathrm{R}}\right) \sum_{k=1}^{1000} \frac{k+1}{(\epsilon+1) k+1}\left(\frac{r}{\mathrm{R}}\right)^{2 k} \mathrm{~d} r
$$

by finding the difference in polarization energy $\Delta E_{\mathrm{pol}}=E_{\mathrm{pol}}\left(\epsilon_{\mathrm{mat}}(\right.$ hexane $\left.)\right)-E_{\mathrm{pol}}\left(\epsilon_{\mathrm{mat}}(\mathrm{AB} 9093)\right)$. Assuming $\mathrm{R}=1.25 \mathrm{~nm}, \epsilon_{\mathrm{QD}}=22.9$ [90], $\epsilon_{\mathrm{mat}}($ hexane $)=1.89$ [91], and $\epsilon_{\mathrm{mat}}(\mathrm{AB} 9093)=4[181]$, $\Delta E_{\mathrm{g}} \approx-8 \mathrm{meV}$ or $\sim 4 \mathrm{~nm}$. Clearly, this is a minor component of the $38 \mathrm{~nm}$ red shift seen in 


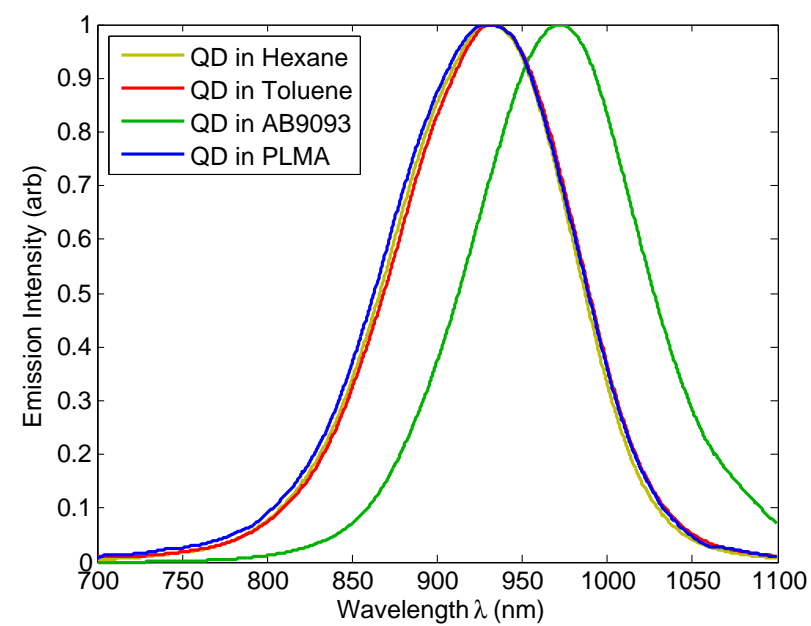

Figure 5.4: Emission spectra of $\mathrm{PbSe}$ QDs in hexane, toluene, AB9093, and P(LMA-co-EGDMA). A large shift in emission peak position is observed for QDs in AB9093, but QDs in the other matrices remain nearly unchanged with respect to hexane.

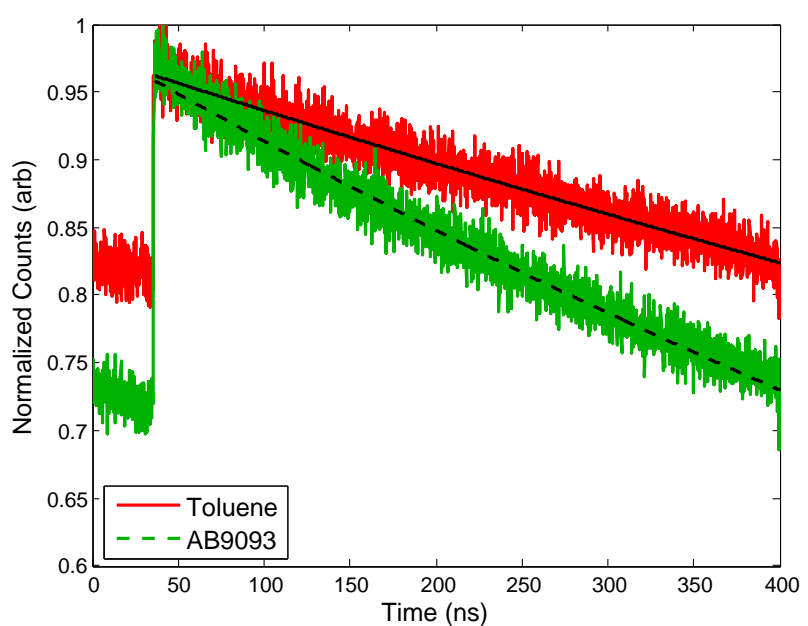

Figure 5.5: Time resolved photoluminescence of QDs in toluene and AB9093, with single exponential fit lines (black).

figure 5.4 when the QD is moved from hexane to AB9093.

Time resolved fluorescence was used to determine the fluorescence lifetime of the QDs in toluene and AB9093, and the results are shown in figure 5.5. The behavior in toluene and AB9093 is single exponential, with lifetimes of $2.34 \mu$ s and $1.34 \mu$ s, respectively. The shortened lifetime in AB9093 is indicative of its lower QY [182]. This is a result of additional non-radiative decay: non-radiative decay becomes increasingly likely compared to radiative decay, decreasing QY and shortening fluorescence lifetime.

\subsection{Conclusion}

PbSe QDs were incorporated into a number of matrix materials. Two materials stood out as candidates for additional testing: AB9093 and P(LMA-co-EGDMA). These two matrices were optically characterized and were both found to be excellent in terms of high optical transmission and low scattering. Mechanically, both AB9093 and P(LMA-co-EGDMA) are very soft, making a glass facing a necessity for handling. 
QDs in AB9093 experienced a large red shift of both the absorption 1-s peak and fluorescence peak, $65 \mathrm{~nm}$ and $38 \mathrm{~nm}$ respectively when compared to hexane. Additionally, the QY of the QDs dropped by about half to $26 \%$. Even so, Pb-salt QDs in a polymer matrix could not be found with a higher QY in literature. Qualitatively, QDs in AB9093 also demonstrate good stability over time and with light exposure. Time resolved fluorescence studies show a faster decay time in AB9093 compared to toluene, indicative of increased non-radiative decay and a lower QY.

QDs in P(LMA-co-EGDMA) experienced a much smaller shift in their optical properties, with a $21 \mathrm{~nm}$ blue shift in 1-s peak and virtually no change to the fluorescence peak. Additionally, there was little or no change to QY upon initial incorporation, but the resultant nanocomposite was sensitive to temporary and permanent photo-damage. 


\section{Chapter 6: Temperature Dependent}

\section{Effects}

The optical properties of QDs change with temperature. This makes them useful as temperature sensors, as noted in section 1.3. However, this change with temperature will affect the operation of LSCs and other devices. Here, PbSe QDs in AB9093 are examined and compared to the same QDs in toluene and Red 305 dye in toluene over a range of temperatures to help determine the effect on LSC performance. QDs in P(LMA-co-EGDMA) were not measured as they were not stable enough under illumination over time. Changing temperatures have the potential to change the optimal LSC configuration, so LSCs could be designed to perform best at a certain expected operating temperature.

\subsection{Optical Absorption Study}

The optical absorption spectra of QDs in AB9093 and toluene are shown in figure 6.1. As annotated on the graphs, there are three regions of interest: wavelengths shorter than the 1-s region, the 1-s peak region, and wavelengths longer than the 1-s region. In the shorter region, there is one peak discernible from the rest. For QDs in AB9093, this peak is at $550 \mathrm{~nm}$ and its intensity decreases at $-0.010 \frac{\%}{{ }^{\circ} \mathrm{C}}$. For QDs in toluene, it is at $523 \mathrm{~nm}$ and drops at $-0.042 \frac{\%}{{ }^{\circ} \mathrm{C}}$.

In both AB9093 and toluene, the magnitude of the QD 1-s peak decreases as temperature increases, as can be seen more clearly in figure 6.2. The decrease is very linear, with the peak of QDs in AB9093 dropping less quickly than those in toluene at $-0.050 \frac{\%}{{ }^{\circ} \mathrm{C}}$ and $-0.083 \frac{\%}{{ }^{\circ} \mathrm{C}}$, respectively. (In this context, percent is not used in a relative sense, which would imply an exponential change. Instead, it is used in the absolute sense.) Finally, in the longer 

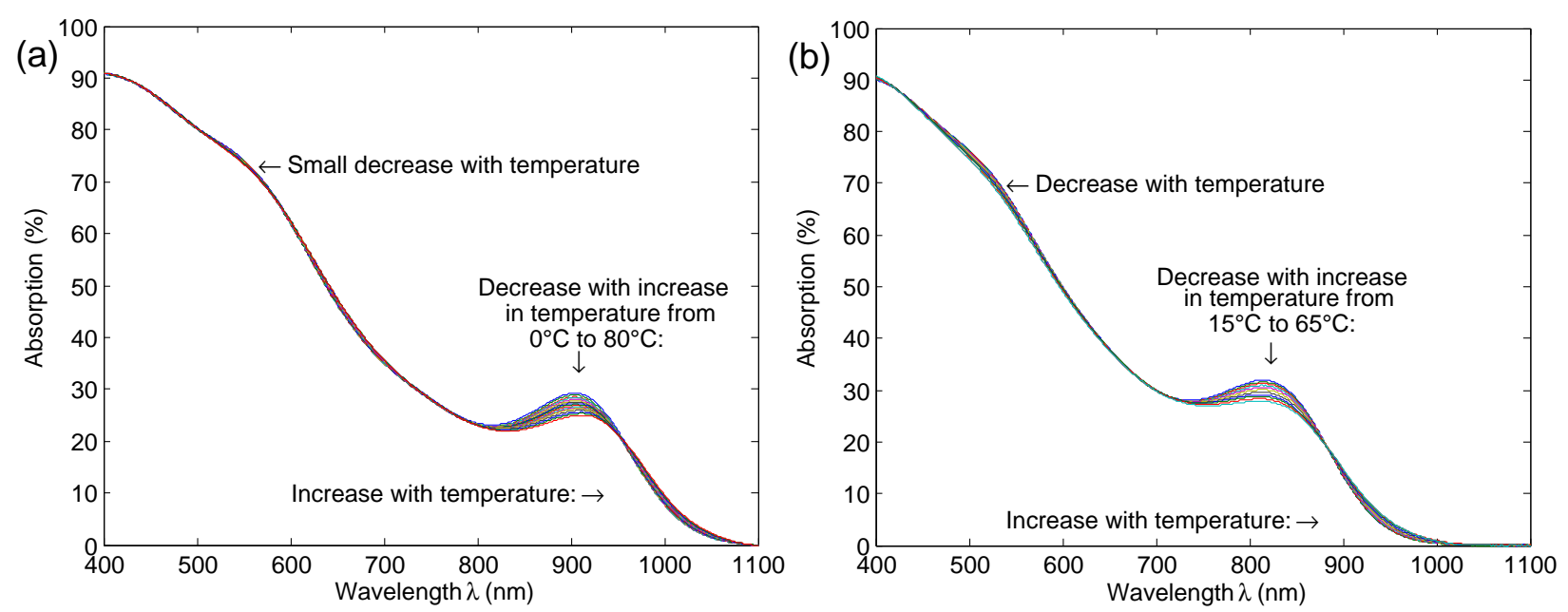

Figure 6.1: Absorption spectra at various temperatures for QDs in (a) AB9093 and (b) toluene. There are three regions of interest noted on each graph.

wavelength region, there is a slight increase with temperature, indicating a broadening of the 1-s peak with temperature.

Less obvious from figure 6.1 is the shift in 1-s peak wavelength, as shown in figure 6.3. The shift in AB9093 is a bi-exponential red shift, totaling about $7.5 \mathrm{~nm}$ from $0^{\circ} \mathrm{C}$ to $80^{\circ} \mathrm{C}$ $\left(\Delta E_{\mathrm{g}}=-11 \mathrm{meV}\right):$

$$
\lambda(T)=895 \exp \left[-1.30 \times 10^{-4} T\right]+9.03 \exp \left[1.31 \times 10^{-2} T\right] .
$$

The shift in toluene is much more complex, stable at first before abruptly blue shifting about $4.5 \mathrm{~nm}$ and stabilizing again $\left(\Delta E_{\mathrm{g}}=8 \mathrm{meV}\right)$.

As noted in section 2.2.5, $\frac{d E_{g}}{d T}$ can be either positive or negative depending primarily on QD size. But, the different dielectric constant of the matrix likely plays a role in the difference seen between AB9093 and toluene. Damage to the QDs also plays a significant role. When cured in AB9093, many of the QDs are damaged, as noted in section 6.2. Smaller QDs, with their proportionately larger surface area, are most likely to be damaged, so the size distribution of the emitting QDs will not be the same in toluene as in AB9093. This is supported by the red shift of the 1-s absorption peak when moving from AB9093 to toluene 
(figure 5.3 and figure 6.1) and, discussed in section 6.2, the fluorescent emission red shift (figure 6.4) and more narrow full-width half maximum of emission of QDs in AB9093 observed as compared to those in toluene (figure 6.7).

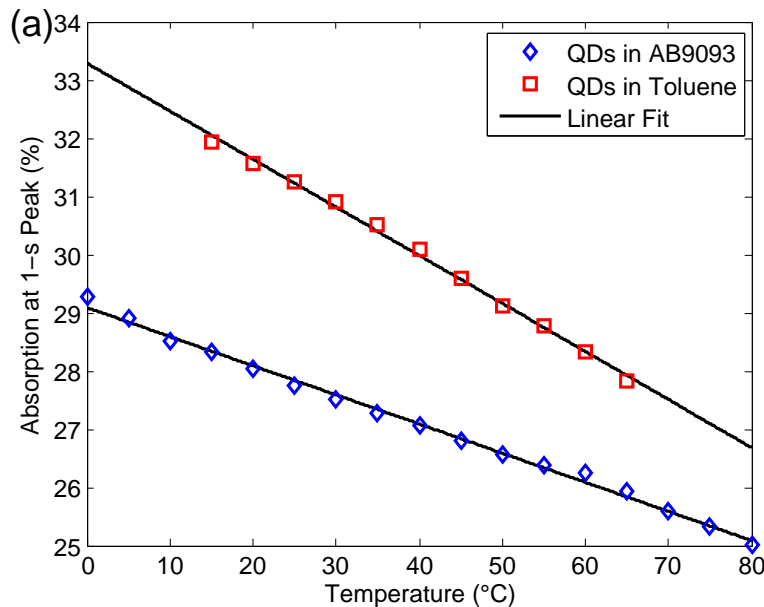

Figure 6.2: QD optical absorption at the 1-s peak linearly decreases with temperature in both AB9093 and toluene.

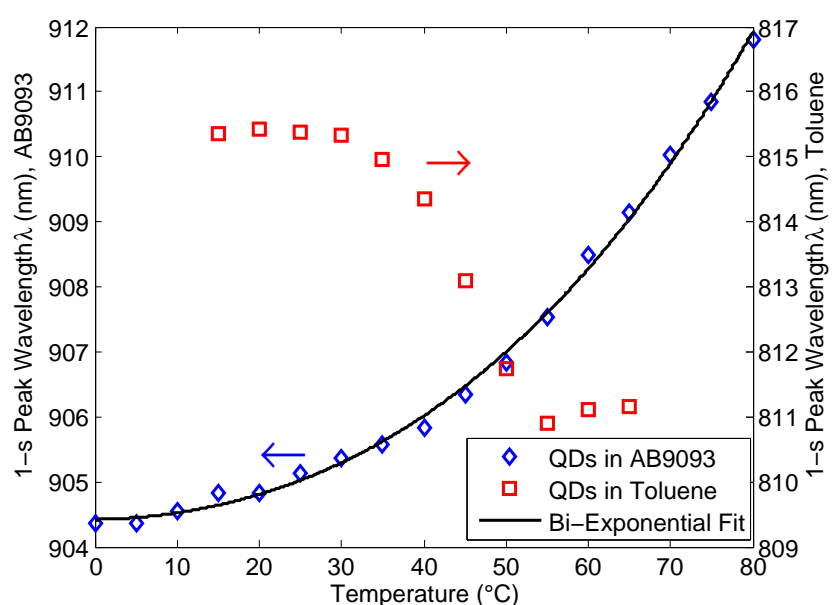

Figure 6.3: The variation of wavelength position of the 1-s peak as a function of temperature. A bi-exponential red shift occurs in AB9093, while the behavior in toluene is complex.

\subsection{QD Fluorescence Study}

Fluorescence intensity, peak position, and full-width at half maximum (FWHM) of QDs were investigated over a temperature range of $5^{\circ} \mathrm{C}$ to $80^{\circ} \mathrm{C}$ for $\mathrm{QDs}$ in $\mathrm{AB} 9093$ and $15^{\circ} \mathrm{C}$ to $65^{\circ} \mathrm{C}$ for QDs in toluene. Each sample was ramped through three thermal cycle runs consecutively to test thermal stability: the sample was brought to $5^{\circ} \mathrm{C}$ or $15^{\circ} \mathrm{C}$, ramped up and measured, cooled, and the process repeated. The fluorescence spectra of QDs in AB9093 and toluene and Red 305 dye in toluene is shown in figure 6.4.

The fluorescent intensity, seen in figure 6.5, is inversely related to temperature. Both reversible and irreversible quenching (a decrease in fluorescence peak intensity) occur as temperature increases. The average fluorescence intensity across three runs drops at a similar 


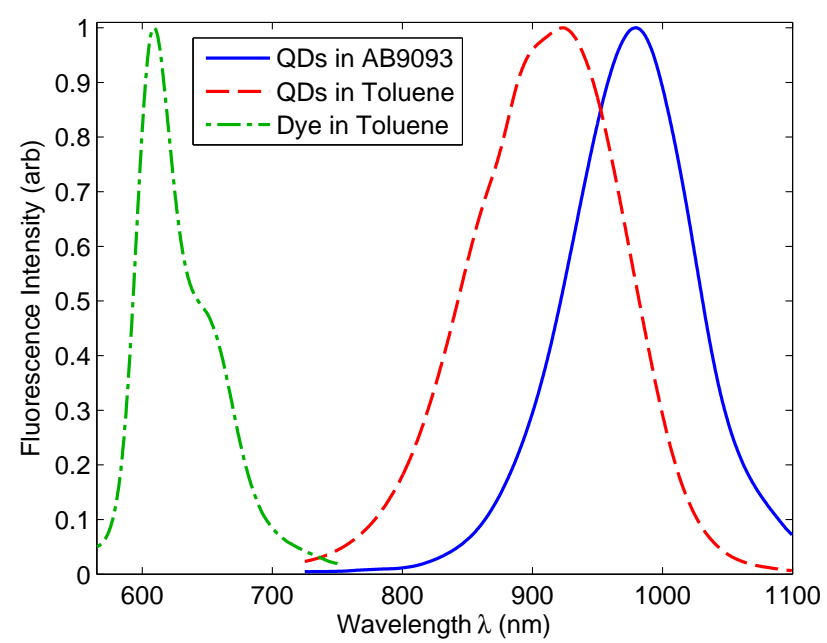

Figure 6.4: The fluorescence spectra of QDs in AB9093 and toluene and Red 305 dye in toluene at $20^{\circ} \mathrm{C}$ during the first data run.

rate for QDs in both $\mathrm{AB} 9093$ and toluene matrices, $-0.94 \frac{\%}{{ }^{\circ} \mathrm{C}}$ for both, implying reversible quenching is not affected by the matrix change.

However, the matrix change does affect irreversible quenching. QDs in AB9093 show very little change between cycles, averaging just $-1.2 \%$ between run 1 and run 2 , and $-2.8 \%$ between run 2 and run 3, totaling -4\% change in fluorescence intensity. But, QDs in toluene average a drop of $-8.55 \%$ between run 1 and run 2 , and $-8.75 \%$ between run 2 and run 3 , totaling -17.3\%. This is because the process of incorporating the QDs into the AB9093 matrix is damaging, evidenced by the initial drop in QY to about half its original value. The individual QDs most vulnerable to damage are thus quenched in AB9093 before the start of the temperature dependent test. Those vulnerable QDs in toluene, on the other hand, are left to be quenched by thermal damage during the test. In comparison, the fluorescence peak intensity of the Red 305 dye is remarkably stable, dropping at about a hundredth of the rate of the QDs with temperature, $-0.01 \frac{\%}{{ }^{\circ} \mathrm{C}}$.

Opposing trends are observed in fluorescence peak position with temperature between QDs in AB9093 and toluene, as shown in figure 6.6, just as with the 1-s absorption peak (figure 6.3). Interestingly, QD fluorescence in each matrix is also the inverse of its absorption trend with temperature. The AB9093 QD fluorescence peak blue shifts $\sim 3 \mathrm{~nm}$ while the 

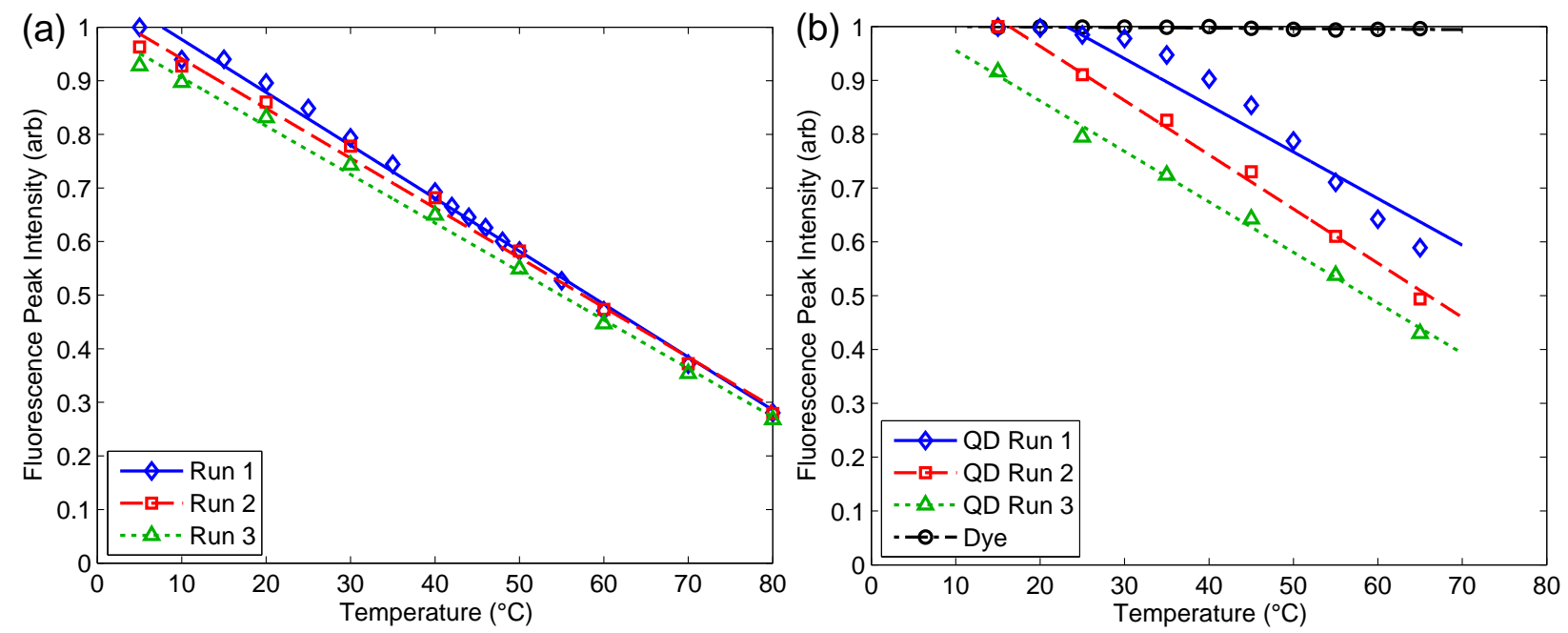

Figure 6.5: Fluorescence intensity change as a function of temperature over three thermal cycles for QDs in (a) AB9093 and (b) toluene. Reversible quenching is about the same for QDs in the two matrices, but irreversible quenching is much greater in toluene. Lines are linear fits.

absorption 1-s peak red shifts $\sim 8 \mathrm{~nm}$ from $5^{\circ} \mathrm{C}$ to $80^{\circ} \mathrm{C}$. QDs in toluene are opposite, with the fluorescence peak red shifting $\sim 8 \mathrm{~nm}$, and the absorption peak blue shifting $\sim 4 \mathrm{~nm}$ from $15^{\circ} \mathrm{C}$ to $65^{\circ} \mathrm{C}$. Thus the apparent Stokes shift of QDs in AB9093 gets smaller with increasing temperature, while in toluene it gets larger. The change in QD fluorescence peak position is also more linear in AB9093 than in toluene, with $\frac{d \lambda}{d T}=0.043 \frac{\mathrm{nm}}{{ }^{\circ} \mathrm{C}}$ and $\frac{d \lambda}{d T}=0.15 \frac{\mathrm{nm}}{{ }^{\circ} \mathrm{C}}$, respectively. Red 305 dye's fluorescence peak shifts at a similar rate with temperature as the QDs at $-0.067 \frac{\mathrm{nm}}{{ }^{\circ} \mathrm{C}}$.

From equation 2.11 in section 2.2.5, it is expected that the full width at half maximum (FWHM) of the QD emission spectra will linearly increase in this temperature range. Though the temperature range of data presented here is insufficient to provide a complete fit to the equation (data near the $0 \mathrm{~K}$ range would be required), it is still predictive of the trend. As seen in figure 6.7, this is true for QDs in AB9093, where $\frac{d \Gamma}{d T}=0.0720 \frac{\mathrm{nm}}{{ }^{\circ} \mathrm{C}}$ or $0.106 \frac{\mathrm{meV}}{\mathrm{K}}$. In toluene, it only holds for run 1 , with $\frac{d \Gamma}{d T}=0.0907 \frac{\mathrm{nm}}{{ }^{\circ} \mathrm{C}}$ or $0.0805 \frac{\mathrm{meV}}{\mathrm{K}}$, before $\frac{d \Gamma}{d T}$ becomes negative in run 2 and run 3.

This abrupt departure from the trend for QDs in toluene can be explained by oxidative damage. This damage can be seen in the higher irreversible quenching with temperature in 

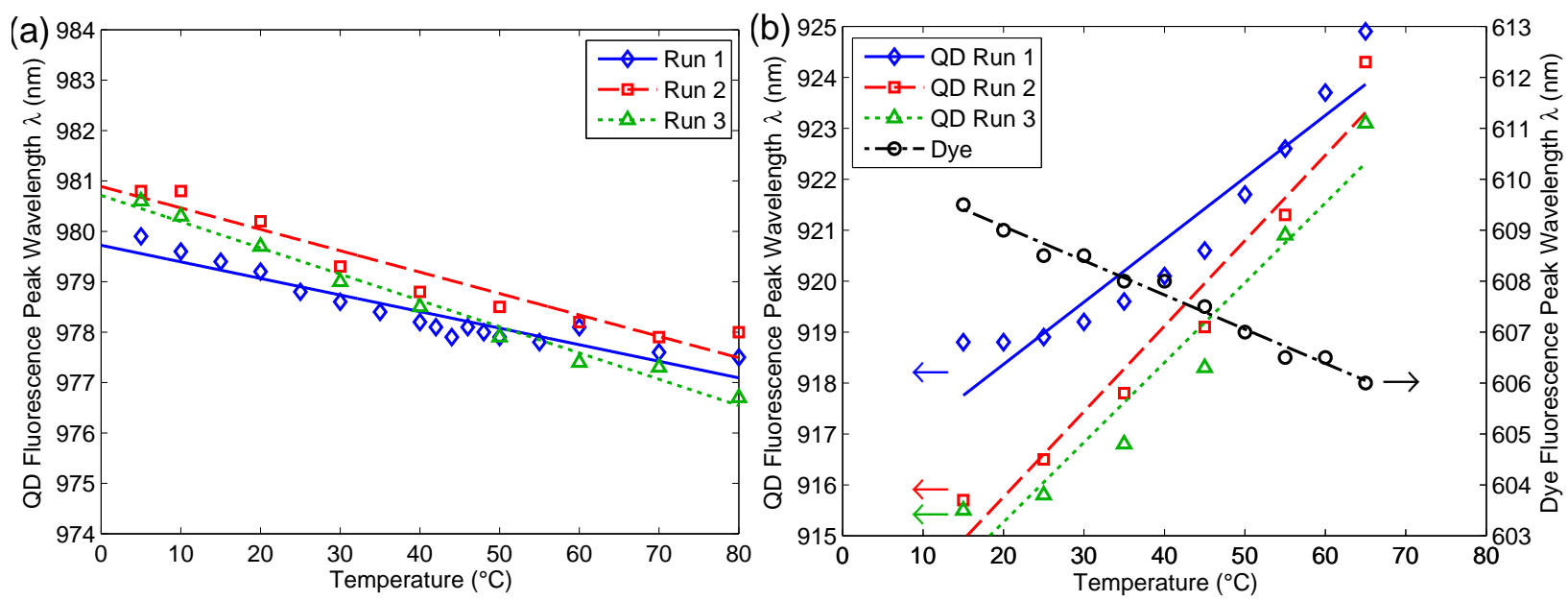

Figure 6.6: Fluorescence peak wavelength shift as a function of temperature over three thermal cycles for QDs in (a) AB9093 and (b) toluene. The trend is both more stable and more linear in AB9093 compared to toluene. QDs in AB9093 and dye in toluene demonstrate a blue shift of fluorescence wavelength with increasing temperature while QDs in toluene red shift. Lines are linear fits.

toluene (figure 6.5b) and the permanent emission blue shifting of QD emission (figure 6.6b). These factors are both characteristic of the QD getting smaller as it oxidizes. Oxidation seems to be a major factor in degradation beginning in the $40^{\circ} \mathrm{C}$ to $50^{\circ} \mathrm{C}$ range. In this temperature range, the 1-s absorption peak shifts rapidly (figure 6.2), the decline of fluorescence intensity begins to occur slightly more rapidly (run one, figure 6.5b), and the fluorescence peak wavelength begins to shift more rapidly (run one, figure 6.6). Thus, data runs two and three were more similar to each other than to run one because the permanent damage happened largely during this first run.

\subsection{Conclusion}

The temperature dependent optical properties of QDs in AB9093 and toluene and dye in toluene were examined in a useful working temperature range for many sensors and devices including LSCs. The results suggest that by keeping the QD nanocomposite under about $40^{\circ} \mathrm{C}$, most permanent change with temperature can be avoided. 

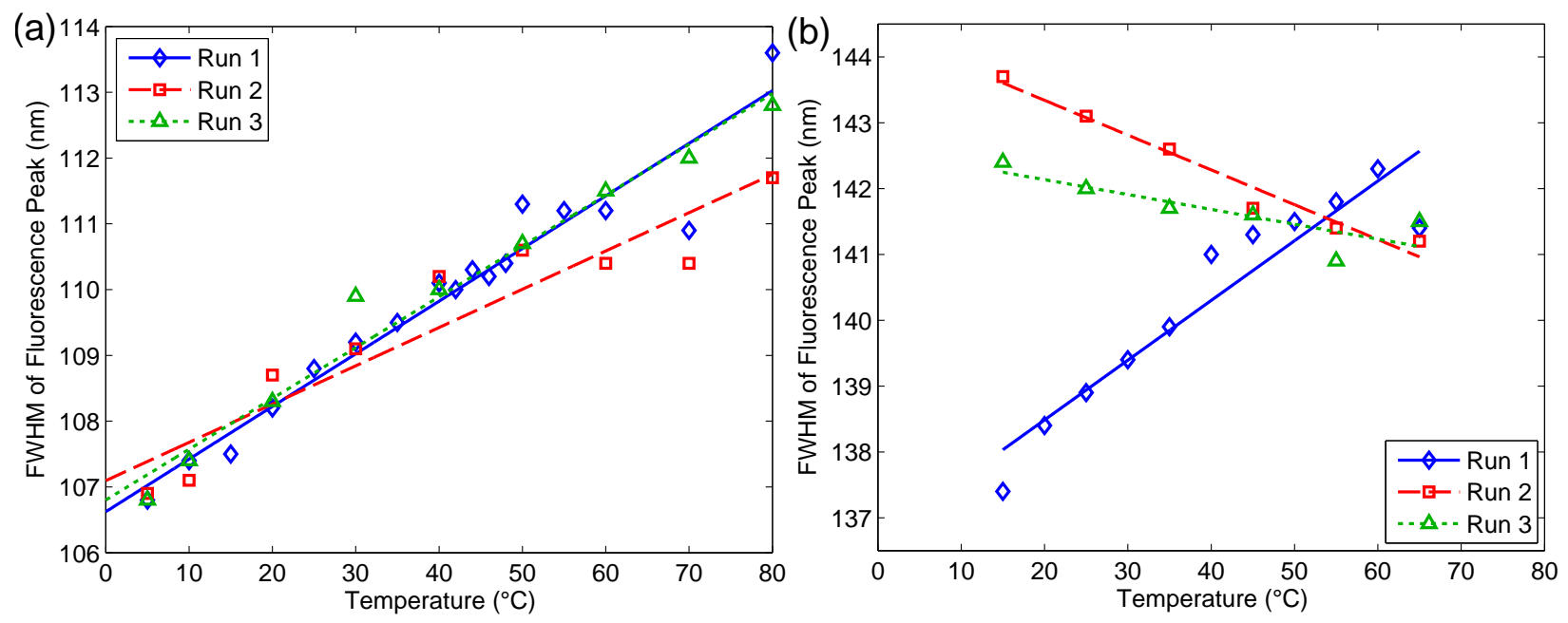

Figure 6.7: Full-width at half maximum of the fluorescence peak with temperature over three thermal cycles for QDs in (a) AB9093 and (b) toluene. Runs 2 and 3 in toluene do not follow the expected trend. Lines are linear fits.

Numerical parameters are summarized in table 6.1, along with comparisons to Cd-salt QD results from literature. While absorption at the 1-s peak is not reported in any of these studies, the change in 1-s peak wavelength with temperature is similar to reported results. Likewise, change in fluorescence intensity, wavelength, and FWHM was similar to reported results. The change in fluorescence intensity, especially, is affected by the measured temperature range, as the rate of change is ultimately non-linear and can vary greatly in different temperature ranges. Change with temperature of the fluorescence wavelength is also greatly affected by the size of the $\mathrm{Pb}$-salt QD. The QDs used here are especially small, $2.5 \mathrm{~nm}$ diameter, and larger dots would have a greater variance with temperature [97].

Greater variation with temperature is observed for some of the parameters for QDs in toluene as compared to those in AB9093. Red 305 dye shows a similar change in fluorescence wavelength as the QDs, but the change in fluorescence intensity with temperature is much less than the rate of QDs. Repeatability of measured parameters upon thermal cycling can be problematic for QDs in toluene, as they are more easily thermally damaged compared to those in AB9093. Interestingly, the trends in change of fluorescence peak wavelength and absorption 1-s peak wavelength are opposites of one another in AB9093 compared to toluene. 
Table 6.1: Measured optical absorption and fluorescence parameters and their change with temperature of QDs in AB9093 and toluene and dye in toluene. Results of other works with Cd-salt QDs are given for comparison; "NR" means the result was not reported by the study. All values were calculated based on linear fits computed across the entire reported temperature range if they were not reported directly in the text.

\begin{tabular}{|c|c|c|c|c|c|c|c|c|c|}
\hline \multirow{2}{*}{\multicolumn{2}{|c|}{ Parameter }} & \multicolumn{3}{|c|}{ This Work } & \multicolumn{5}{|c|}{ Other Works } \\
\hline & & AB9093 & Toluene & Dye & (a) & (b) & (c) & (d) & (e) \\
\hline 1-s Abs. & $\frac{\%}{{ }^{\circ} \mathrm{C}}$ & -0.050 & -0.083 & & NR & NR & NR & NR & NR \\
\hline 1-s Peak $\lambda$ & $\frac{n m}{{ }^{\circ} \mathrm{C}}$ & 0.09 & -0.09 & & 0.07 & NR & 0.105 & NR & NR \\
\hline Fluor. Intensit & $\frac{\%}{{ }^{\circ} \mathrm{C}}$ & -0.94 & -0.94 & -0.01 & -0.43 & -0.173 & -0.6 & -1.47 & -3.9 \\
\hline Fluor. $\lambda$ & $\frac{\mathrm{nm}}{{ }^{\circ} \mathrm{C}}$ & -0.043 & 0.15 & -0.067 & 0.11 & 0.145 & 0.108 & 0.193 & 0.25 \\
\hline FWHM & $\frac{n m}{{ }^{\circ} \mathrm{C}}$ & 0.0720 & 0.091 & & NR & $\mathrm{NR}$ & 0.076 & $\mathrm{NR}$ & NR \\
\hline Temperature & ${ }^{\circ} \mathrm{C}$ & $0-80$ & $15-65$ & $15-65$ & $22-252$ & $-40-70$ & $30-180$ & $23-80$ & $25-50$ \\
\hline \multicolumn{10}{|c|}{$\begin{array}{ll}\text { (a) } \mathrm{CdSe} / \mathrm{ZnS} \text { in } \mathrm{SiO}_{2}[93] & \text { (d) } \mathrm{CdTe} \text { in layered double hydroxide [183] } \\
\text { (b) CdSe in optical fiber [184] } & \text { (e) CdTe in poly(methyl methacrylate) [31] } \\
\text { (c) } \mathrm{CdSe} / \mathrm{ZnS} \text { in } \mathrm{SiO}_{2}[112] & \end{array}$} \\
\hline
\end{tabular}

In AB9093, many parameters, including 1-s absorption, fluorescence intensity, fluorescence wavelength, and FWHM, are linear and repeatable with changing temperature, making them appropriate for the basis of a nanocomposite sensor. The 1-s absorption peak wavelength is also well fit with a bi-exponential function.

The greatest concern for an LSC is the drop in fluorescence intensity with increasing temperature. At $40^{\circ} \mathrm{C}$, the highest temperature likely to be regularly encountered by most LSC installations, the fluorescence intensity drops to about $77 \%$ of its value at $20^{\circ} \mathrm{C}$. The dye retains over $99 \%$ of its $20^{\circ} \mathrm{C}$ fluorescence intensity at $40^{\circ} \mathrm{C}$. This compares to a $\mathrm{Si} \mathrm{PV}$ cell which retains about $94 \%$ of its $20^{\circ} \mathrm{C}$ efficiency at $40^{\circ} \mathrm{C}$ (calculated by a mean of linear fits to the data given by Singh and Ravindra [185]). 


\section{Chapter 7: Luminescent Solar}

\section{Concentrators}

QD LSCs were fabricated and tested as discussed in chapter 4 and throughly optically characterized at room temperature and over a range of temperatures in chapters 5 and 6 , respectively. Here, the performance of complete QD LSC PV systems will be discussed. The primary metric of evaluation is the power conversion efficiency, PCE, formally defined as:

$$
\mathrm{PCE} \equiv \frac{P_{\text {elec }}}{P_{\mathrm{opt}}}=\frac{2 P_{\mathrm{mpp}}}{100 \frac{\mathrm{mW}}{\mathrm{cm}^{2}} \cdot L_{\mathrm{LSC}} W_{\mathrm{LSC}}}
$$

where $P_{\text {elec }}$ is the electrical power produced by the LSC PV system, $P_{\text {opt }}$ is the optical power incident on the LSC $\left(100 \frac{\mathrm{mW}}{\mathrm{cm}^{2}}\right), P_{\mathrm{mpp}}$ is the electrical power produced by a single PV cell at the maximum power point, and $L_{\mathrm{LSC}}$ and $W_{\mathrm{LSC}}$ are the length and width of the LSC in $\mathrm{cm}$. $P_{\mathrm{mpp}}$ is doubled here because there are two solar cells attached to the LSCs in this work.

Additionally, fill factor $F F$ can be computed as follows:

$$
F F=\frac{V_{\mathrm{mpp}} I_{\mathrm{mpp}}}{V_{\mathrm{oc}} I_{\mathrm{sc}}}
$$

where $V_{\text {mpp }}$ and $I_{\text {mpp }}$ are the voltage and current at the maximum power point and $V_{\text {oc }}$ and $I_{\mathrm{sc}}$ are the open circuit voltage and short circuit current.

\subsection{Initial Tests}

Initial tests were used to improve later results through an iterative testing and evaluation process with enhancements of fabrication and testing methods. Because the testing setup 
was changed, these results cannot be directly compared with later results. A fabricated LSC and a complete LSC PV system are shown in figure 7.1.
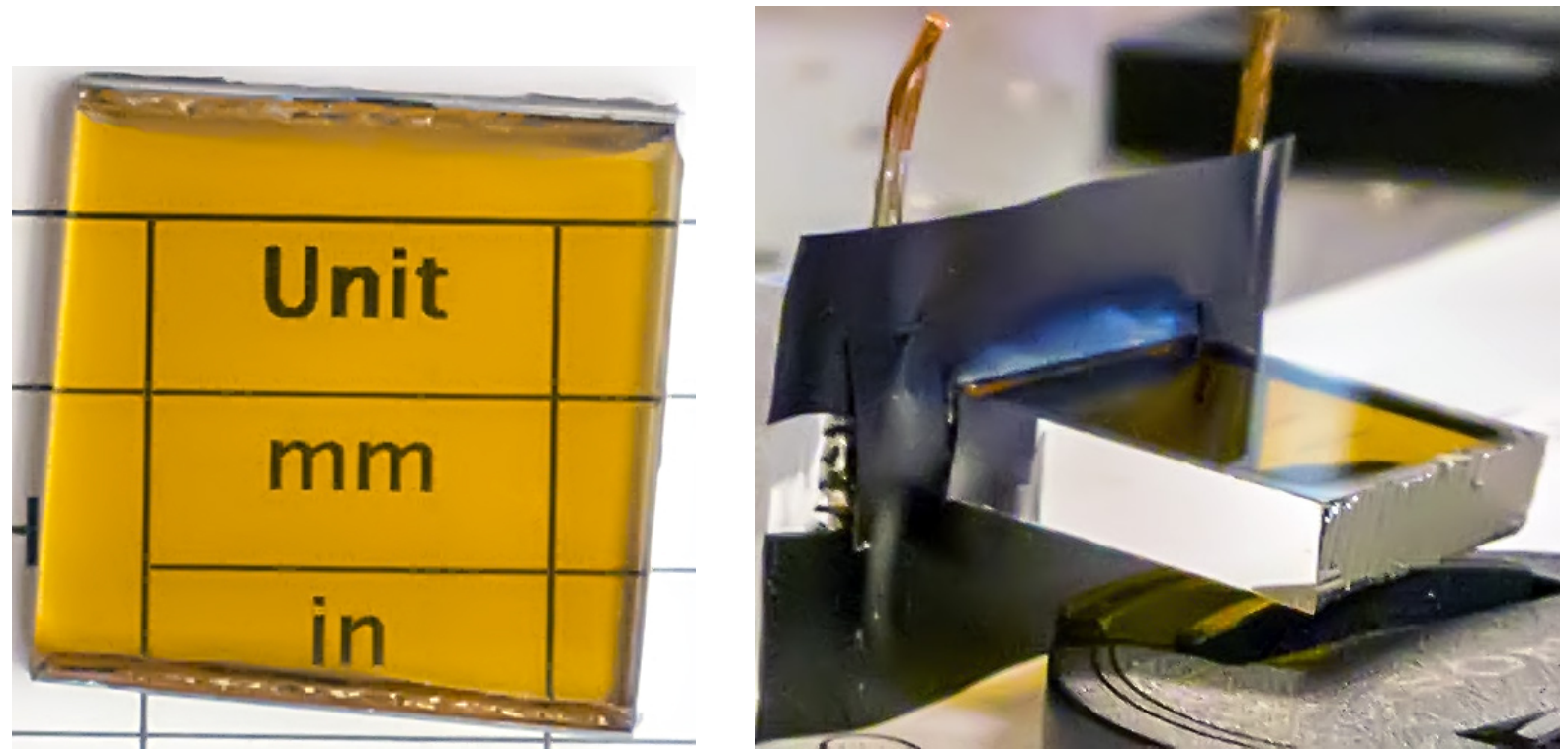

Figure 7.1: Left: A fabricated LSC sample (P(LMA-co-EGDMA) matrix). It is easy to read text through the LSC sample because of its high optical quality. Sample shown is approximately $2 \mathrm{~cm} \times 2 \mathrm{~cm}$. Right: Complete LSC system with PV cell attached. Any excess PV cell area is masked from light exposure.

First, an LSC was fabricated with an AB9093 matrix. From a materials standpoint, it is a better matrix material than $\mathrm{P}$ (LMA-co-EGDMA) because of its higher refractive index $(n=1.56)$ and slightly lower absorption, especially in the near IR region. However, from earlier characterization it was known to interact with the QDs in a detrimental way, lowering the QY by about half. Some selected results of testing with an AB9093 matrix are shown in table 7.1 .

Although the measured PCE was much lower than the simulation detailed in chapter 3 predicted should be possible, the trends seen in modeling were observed in experiment. The simulation predicts a rapid increase in PCE as $N$ rises from zero, before reaching a peak and falling off less rapidly. This can be seen in table 7.1. When $N$ was "optimized," i.e. the PCE is greatest for the particular LSC system, the PCE was 1.97\%. When an LSC device was fabricated with an $N 12.5 \%$ less, the PCE falls by $-21.3 \%$, relatively. The PCE of a device 
fabricated with an N 12.5\% higher was tested and the PCE fell less quickly, as expected, by $-6.1 \%$.

Table 7.1: Measured parameters from initial AB9093 LSC testing at various QD concentrations $N$, where the optimized $N$ is the experimental result with the best PCE.

\begin{tabular}{|ll|ccc|}
\hline & & Optimized $N$ & $N-12.5 \%$ & $N+12.5 \%$ \\
\hline$V_{\text {oc }}$ & $\mathrm{mV}$ & 547.4 & 537.4 & 540.3 \\
$I_{\mathrm{sc}}$ & $\mathrm{mA}$ & 11.32 & 8.82 & 10.16 \\
$V_{\mathrm{mpp}}$ & $\mathrm{mV}$ & 426.6 & 417.2 & 422.7 \\
$I_{\mathrm{mpp}}$ & $\mathrm{mA}$ & 9.72 & 7.67 & 8.84 \\
$F F$ & $\%$ & 66.9 & 67.5 & 68.1 \\
PCE & $\%$ & 1.97 & 1.55 & 1.85 \\
length & $\mathrm{cm}$ & 2.1 & 2.1 & 2.0 \\
width & $\mathrm{cm}$ & 2.1 & 2.0 & 2.0 \\
\hline
\end{tabular}

P(LMA-co-EGDMA) QD LSC samples were also fabricated and tested. Although the matrix absorption is higher and $n$ is only 1.45, the QDs do not suffer as much damage when incorporated into this material. As seen in table 7.2, this results in a higher overall PCE, in spite of the material disadvantages.

Table 7.2: Measured parameters from initial P(LMA-co-EGDMA) LSC testing at various QD concentrations $N$, where the optimized $N$ is the experimental result with the best PCE.

\begin{tabular}{|ll|ccc|}
\hline & & Optimized $N$ & $N-15 \%$ & $N+15 \%$ \\
\hline$V_{\text {oc }}$ & $\mathrm{mV}$ & 553.7 & 554.0 & 561.0 \\
$I_{\text {sc }}$ & $\mathrm{mA}$ & 20.29 & 15.71 & 16.1 \\
$V_{\mathrm{mpp}}$ & $\mathrm{mV}$ & 423.4 & 425.6 & 441.0 \\
$I_{\mathrm{mpp}}$ & $\mathrm{mA}$ & 16.87 & 13.3 & 13.74 \\
$F F$ & $\%$ & 63.4 & 65.0 & 66.8 \\
PCE & $\%$ & 3.37 & 2.75 & 2.97 \\
length & $\mathrm{cm}$ & 2.1 & 2.0 & 2.0 \\
width & $\mathrm{cm}$ & 2.1 & 2.0 & 2.0 \\
\hline
\end{tabular}

Again, as the concentration $N$ moves away from the ideal point, the PCE drops faster below the ideal point than above, $-18.4 \%$ and $-11.9 \%$ relatively, respectively. While the P(LMA-co-EGDMA) LSC system outperformed the AB9093 system by $71.1 \%$, relatively, these results were still below what was expected based on the model. 


\subsection{Best QD LSC}

After a careful examination of the results of the initial testing, numerous improvements were made to the testing setup and samples. The illumination from the original test setup was found to be too divergent to provide even illumination of the sample, and the intensity was therefore on average too low. The improved testing setup was calibrated by measuring a solar cell of the same dimensions as the LSC top face. The measured parameters from this test are shown in table 7.3. $I_{\mathrm{sc}}$ closely matched the specified value, but $V_{\text {oc }}$ was much lower, perhaps from damage during the cell preparation process. This reduced the performance of the PV cell and the resultant LSC.

Both the PV cell and mirrors were improved as well. Previous tests used a PV cell specified at $16.5 \%$ efficiency with front metal finger contacts. These were replaced with cells specified at $22 \%$ efficiency with no opaque front contacts. The side mirrors on previous samples were reflective Mylar tape with an acrylic adhesive. The reflectance from this tape was in the range of $75-85 \%$, depending on wavelength. As highlighted by the simulation in section 3.4 , such a low mirror reflectivity greatly reduces the maximum $\eta_{\text {opt }}$ able to be achieved by the system. This was replaced with a polymer film, $R \geq 97 \%$ over all wavelengths

of interest. Finally, the diffuse back reflector was moved as close to the underside of the sample as possible while maintaining an air gap; previously it was spaced about $2 \mathrm{~mm}$ from the LSC.

Based on the previous tests, a new P(LMA-co-EGDMA) sample was constructed with the above improvements and tested. The concentration of QDs used was the same as the ideal concentration for the previous test, $\sim 12.7 \mu \mathrm{M}(\sim 3 \mathrm{ppm}$ by weight). The results are shown in table 7.3, and the I-V curve is shown in figure 7.2. The PCE of this sample was measured to be $4.93 \%$, below the predicted $6.07 \%$. This sample has the highest reported PCE for any LSC using either QDs or silicon PV cells in its construction, and a higher optical efficiency $\left(\eta_{\mathrm{opt}}=0.26\right)$ than the state of the art dye system $\left(\eta_{\mathrm{opt}}=\sim 0.2\right)$. If the $V_{\mathrm{oc}}$ of the PV cells 
Table 7.3: Measured parameters for the Si PV cell and the most efficient P(LMA-co-EGDMA) QD LSC. Values measured for the LSC with the lamp used in this work (OSL1) are shown alongside values measured in another lab with a simulated solar lamp calibrated with an NREL certified cell (Solar Sim.) for verification of the results of this work. NREL certified values from literature for the type of PV cell used are shown in parenthesis [47]; manufacturer specified values for a module are given in square brackets [186].

\begin{tabular}{|ll|cc|cc|}
\hline & & \multicolumn{2}{|c|}{ PV Cell } & \multicolumn{2}{c|}{ LSC } \\
& & Measured & Specified & OSL1 & Solar Sim. \\
\hline$V_{\mathrm{oc}}$ & $\mathrm{mV}$ & 600.0 & $(724.7)$ & 544.0 & 560.3 \\
$I_{\mathrm{sc}}$ & $\mathrm{mA}$ & 169.6 & $(170.2)$ & 27.60 & 26.53 \\
$V_{\mathrm{mpp}}$ & $\mathrm{mV}$ & 471.5 & {$[569.8]$} & 428.9 & 426.8 \\
$I_{\mathrm{mpp}}$ & $\mathrm{mA}$ & 152.6 & {$[157.3]$} & 24.02 & 23.2 \\
$F F$ & $\%$ & 70.7 & $(80.1)$ & 68.13 & 66.5 \\
PCE & $\%$ & 17.12 & $(22.4 \pm 0.6)$ & 4.93 & 4.74 \\
length & $\mathrm{cm}$ & 2.1 & 2.1 & 2.1 & 2.1 \\
width & $\mathrm{cm}$ & 2.1 & 2.1 & 2.0 & 2.0 \\
\hline
\end{tabular}

used matched specifications, the PCE of the system might be as high as $6 \%$ with the same optical efficiency.

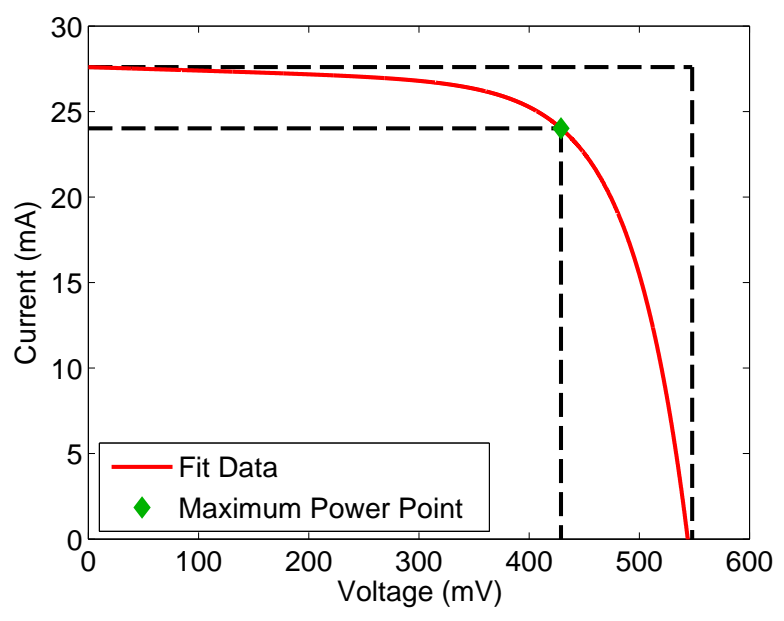

Figure 7.2: I-V curve of the best performing P(LMA-co-EGDMA) QD LSC. Dotted lines are a guide to the eye for important voltages and currents.

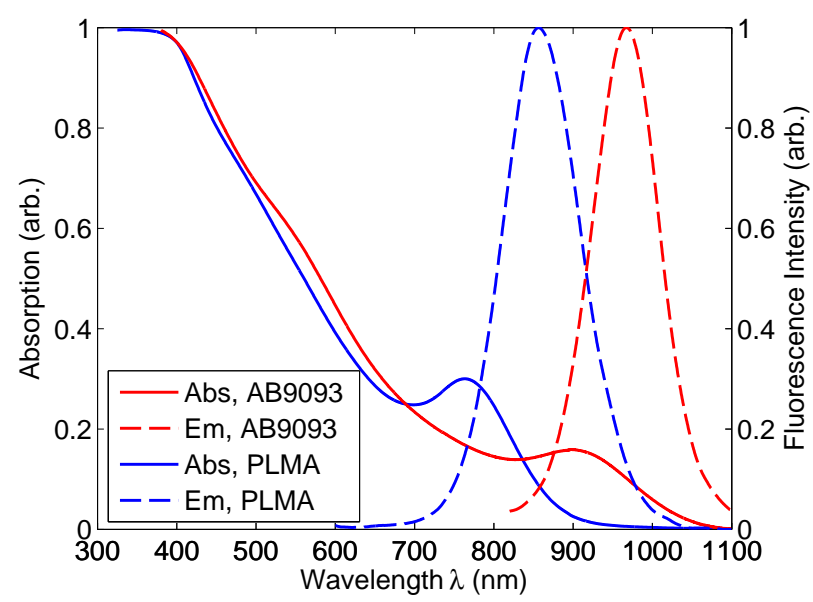

Figure 7.3: QD optical absorption and fluorescence in the AB9093 and P(LMA-co-EGDMA) LSCs.

To verify the results of this work, the best LSC sample was sent to another lab and measured by another researcher in our group. This lab makes use of a lamp which closely replicates the AM1.5 solar spectrum; the intensity was calibrated with an NREL certified 
test PV cell. The results are shown in the right-most column of table 7.3 [187]. The PCE is slightly lower when measured under this solar simulator lamp, $4.74 \%$ instead of $4.93 \%$, because the spectrum of the OSL1 lamp used in this work is absorbed more fully by the QDs (i.e. the OSL1 lamp spectrum is more favorably matched than the solar simulation lamp to the optical absorption spectrum of the QDs).

One immediate route forward to even higher PCE can be seen in by examining the QD optical spectra in figure 7.3. By synthesizing a slightly larger QD, its band gap and thus absorption and fluorescence spectra could be red shifted and matched more closely to the Si PV cell band gap. This would in turn allow more solar absorption. Again in this respect AB9093 seems to have an advantage because it causes a red shift of the QD spectra. However, not only is the QY much lower in AB9093, the Stokes shift is reduced from $93 \mathrm{~nm}$ in $\mathrm{P}$ (LMA-co-EGDMA) to $70 \mathrm{~nm}$ in AB9093. This results in additional self absorption and reduced $\eta_{\text {opt }}$.

\subsection{Conclusion}

PbSe QD LSCs were fabricated and optimized based on simulation and an iterative testing process. The best result, $\mathrm{PCE}=4.93 \%$, was achieved using a P(LMA-co-EGDMA) matrix and high quality reflectors. The PCE was verified using a lamp which closely matches the AM1.5 solar spectrum in another lab, resulting in $\mathrm{PCE}=4.74 \%$. This represents the highest reported PCE of any LSC using either QDs or silicon PV cells. As compared to the state of the art dye LSC with PCE $=7.1 \%$, the QD LSC has a higher $\eta_{\text {opt }}$. However, the dye LSC is able to take advantage of a much higher PV cell $V_{\text {oc }}=1 \mathrm{~V}$, nearly double the $V_{\text {oc }}=0.544 \mathrm{~V}$ measured in this work. If the silicon PV cell $V_{\text {oc }}$ was raised to the specified $725 \mathrm{mV}$, the PCE of the LSC system in this work would be raised to $\sim 6 \%$.

The most significant loss still present in this system is QY loss. By increasing the QD QY to 0.9 from 0.7 , the PCE is predicted to rise from $4.93 \%$ to $\sim 7 \%$. If this is combined 
with the $V_{\text {oc }}$ increase discussed above, the PCE would be $>9 \%$. Another large loss is escape cone. This can be decreased with a wavelength selective reflector on the top surface of the LSC. Simulation predicts a relative $25 \%$ increase in $\eta_{\text {opt }}$ upon application of a top mirror having $R=0$ at $\lambda<774 \mathrm{~nm}$ and $R=0.8$ at $\lambda \geq 775 \mathrm{~nm}$. 


\section{Chapter 8: Conclusions \& Future}

\section{Work}

\subsection{Conclusions}

In this work, PbSe QDs were examined as a replacement for dyes as the luminophore in LSCs because of their wide absorption spectrum and potential for improvements in performance and long-term stability. A thorough theoretical understanding of losses in an LSC system was provided. Because each loss is difficult to decouple from other losses, their effect individually on the system as a whole is difficult to quantify within a theoretical framework. For example, the ideal matrix index of refraction can be found analytically from examining the balance between front surface reflection and the fraction of photons unable to be captured by total internal reflection, but this does not account for a back reflector to reflect light back into the LSC. The ideal index further depends on a number of other factors, such concentration and QY of the luminophore in the matrix.

To quantify and further understand these losses for a given LSC system, a Monte Carlo ray tracing model was written. This state of the art model was verified against the experimental results in this work as well as those in literature, and any discrepancies were explained. It is capable of simulating an LSC system with a great number of user defined input parameters, including arbitrary luminophore and incident light spectra. Many outputs are available to the user, including a detailed breakdown of losses, the LSC output spectrum, photon output angles, and average distance traveled by the photons. This provides a much more complete understanding of the effect of some variable on the LSC performance than predicting only $\eta_{\mathrm{opt}}$.

Next, an appropriate matrix material was found to host the QDs. This was not trivial, due 
to the many requirements on such a material. First, it must be optically clear in the visible and near IR region so it can act as a waveguide. Second, it must be chemically compatible with the QDs: they must disperse within the material well, and it must not alter their optical properties to a great extent, or cause damage reducing the fluorescence QY. Third, curing and processing of the material must not require any steps which might damage the QDs, such as excessive heat. Finally, it must protect the QDs and preserve their luminescence from the deleterious effects of oxygen, high energy photons, and the environment.

Given these requirements, two polymer materials were identified for further study, AB9093 UV curing epoxy and the co-polymer P(LMA-co-EGDMA). Both were extensively optically characterized with and without QDs at room temperature and found to possess excellent optical qualities. QDs in P(LMA-co-EGDMA) displayed less of a change in optical properties than those in AB9093, and also exhibited far less quenching of luminescence. Unfortunately both polymers, like nearly all polymers, absorb slightly in the near IR emission spectral region of the QDs due to harmonics of the carbon-hydrogen bond.

QDs in AB9093 were further studied over a range of temperatures and compared to QDs in toluene. The effect of temperature on many optical properties of the QDs were investigated, including the 1-s absorption peak wavelength and intensity, and the fluorescence peak intensity, wavelength, and FWHM. This variation with temperature points to the possibility of using the QD nanocomposite as a sensor. Most changes were of little consequence for an LSC application, except for the change in fluorescence intensity. At $40^{\circ} \mathrm{C}$, near the upper range of temperature one might expect an LSC to be exposed to, the fluorescence intensity was found to drop to about $77 \%$ its value at $20^{\circ} \mathrm{C}$. For comparison, literature results were used to calculate that Si PV cells retain about $94 \%$ of their $20^{\circ} \mathrm{C}$ efficiency at $40^{\circ} \mathrm{C}$.

Using the results of modeling and characterization, an LSC PV system was fabricated. After an iterative optimization process, the best sample, QDs in P(LMA-co-EGDMA), was measured to have a broadband PCE $=4.93 \%$ (4.74\% using a lamp closely matching AM1.5 solar illumination). This represents the state of the art for any LSC system using QDs or 
Si PV cells in their construction. $\eta_{\mathrm{opt}}$ was found to be higher for this system, $\eta_{\mathrm{opt}} \approx 0.26$, than the overall state of the art LSC system employing a combination of two dyes, $\eta_{\mathrm{opt}} \approx 0.2$. However, the much lower $V_{\text {oc }}$ of the Si PV cells compared to the GaAs PV cells used for the state of the art LSC limited the PCE of the system described in this work.

Based on a simplistic cost analysis assuming $\$ 2 / \mathrm{kg}$ for polymer matrix material and a $0.5 \mathrm{~cm}$ thick LSC with a geometric gain $G=2$ as in the current work, a PCE of about $9 \%$ would reach cost parity with a PV system with $18 \%$ efficient cells at $\$ 0.75 / \mathrm{W}$ with a single axis tracker adding $\$ 0.15 / \mathrm{W}$. An LSC with $G=5$ would reach cost parity at a PCE of just $4.5 \%$. Even before reaching cost parity, they are an attractive option for building integrated LSC systems $[19,188,189]$ because there are some locations where traditional PV systems cannot be deployed for practical or aesthetic reasons. LSCs can be used as a colorful building façade, in place of windows or skylights, or as a flexible thin film coating on nearly any surface. One such thin film has been demonstrated with $\eta_{\mathrm{opt}}=13 \%$ for use as a window coating or building wrap [188].

In order to reach cost parity and better, a few major losses must be addressed. First, increasing QY to $>0.9$ alone would increase the LSC PCE to $~ 7 \%$, according to simulated values and experimental results. This is realistic for the very near future. Escape cone loss could be greatly reduced with a wavelength-selective mirror on the top surface, with simulation predicting a relative improvement in $\eta_{\mathrm{opt}}$ of $25 \%$. Finally, smaller improvements such as better matching the QD emission with the solar cell band gap, optimizing the PV cell ARC, and reducing TIR loss would also help to drive up system efficiency.

\subsection{Future Work}

Though state of the art, there are numerous improvements which could be made to the Monte Carlo LSC simulation. The biggest improvement to the simulation package would be the addition of scattering effects from the matrix and luminophores. More complicated 
luminophore effects such as aggregation at high concentration, FRET-based concentration luminescence quenching, and deviation from the Beer-Lambert law could be modeled with additional careful experimental characterization of the luminophore and matrix material. A finite air gap could be added between the LSC and back reflector. Currently, although the simulation acts as if there is an air gap, it is treated as infinitesimally small, such that the photon position is unchanged between the LSC and bottom mirror. Photons could also be allowed to enter the LSC at any angle instead of always normal to the LSC face. Finally, if the simulation were to be used extensively for a luminophore with a multi-modal fluorescence spectrum, allowed absorption to fluorescence transitions would need to be tracked in order to properly predict the LSC output spectral shape.

The LSC could also be improved in a number of ways. Perhaps the easiest way is to better match the emission of the QD to the band gap of the Si PV cell to maximize the solar spectrum absorbed at the lowest possible QD concentration. This could be done by synthesizing slightly larger QDs. More difficult synthesis tasks include maximizing the fluorescence QY and ensuring stability over time. The Stokes shift could also be increased to limit self absorption related losses. Many techniques for accomplishing these goals (discussed in section 1.1.4) have been successfully applied for CdSe QDs, which enjoy much greater popularity in scientific literature.

QDs can also be engineered for improved performance in a variety of other ways. Multiple exciton generation, or MEG, is a phenomenon whereby a single incident photon can excite multiple electrons within the QD, and has been demonstrated in PbSe QDs [75]. Plasmonic effects can also be investigated. Many groups have observed greatly enhanced luminescence intensity of QDs by taking advantage of plasmonic interactions [190-193].

Fabrication techniques could also be improved. A relatively low $V_{\text {oc }}$ prevented much higher PCE in this work. This was perhaps at least partially a result of damage to the PV cell during preparation for system fabrication because the cells had to be broken from larger wafers. Better bonding and optical coupling between the LSC and PV cell would also 
enhance performance. Voids between the LSC and PV cell were an ever-present issue, and the refractive indices of the dielectric stack could be optimized for lesser surface reflection. Various other LSC geometries like circles have shown higher $\eta_{\mathrm{opt}}$, but they may prove difficult to mate with a PV cell unless less efficient flexible cells are used. A wavelength selective top mirror would also improve $\eta_{\mathrm{opt}}$.

Other matrix materials should also be investigated. As the LSC is scaled up, transport losses like matrix absorption become even more significant. Near IR matrix absorption must be reduced or eliminated before a large-scale LSC based on PbSe QDs can be fabricated with high $\eta_{\text {opt }}$. This is difficult to achieve with polymers, as the absorption is caused by the carbon-hydrogen bond making up the "back bone" of most polymers. Chemically treated deuterated polymers are one possibility [194]. 


\section{Bibliography}

[1] G. Masson, S. Orlandi, and M. Rekinger, "Global market outlook for photovoltaics 2014-2018," European photovoltaic industry association, 2014. [Online]. Available: http://www.epia.org/fileadmin/user_upload/Publications/ EPIA_Global_Market_Outlook_for_Photovoltaics_2014-2018_-_Medium_Res.pdf

[2] US Dept. of Energy. (2005) Basic research needs for solar energy utilization. [Online]. Available: http://science.energy.gov/ /media/bes/pdf/reports/files/seu_rpt.pdf

[3] W. H. Weber and J. Lambe, "Luminescent greenhouse collector for solar radiation," App. Opt., vol. 15, no. 10, pp. 2299-300, 1976.

[4] A. Goetzberger and W. Greubel, "Solar energy conversion with fluorescent collectors," Appl. Phys., vol. 14, pp. 123-39, 1977.

[5] S. J. Smith, "PV trackers," Solarpro, vol. June/July, p. 36, 2011. [Online]. Available: http://nextphasesolar.com/wp-content/uploads/2011/12/Solar-Pro_PV-Trackers.pdf

[6] NREL, "Reference solar spectral irradiance: ASTM G-173." [Online]. Available: http://rredc.nrel.gov/solar/spectra/am1.5/ASTMG173/ASTMG173.html

[7] A. Goetzberger, "Fluorescent solar energy concentrators: operating conditions with diffuse light," Appl. Phys., vol. 16, pp. 399-404, 1978.

[8] S. J. Gallagher, B. C. Rowan, J. Doran, and B. Norton, "Quantum dot solar concentrator: Device optimisation using spectroscopic techniques," Sol. Energy, vol. 81, pp. 540-7, 2007.

[9] B. C. Rowan, L. R. Wilson, and B. S. Richards, "Advanced material concepts for luminescent solar concentrators," IEEE J. Sel. Topics Quantum Electron., vol. 14, no. 5, pp. 1312-22, 2008.

[10] L. H. Slooff, E. E. Bende, A. R. Burgers, T. Budel, M. Pravettoni, R. P. Kenny, E. D. Dunlop, and A. Büchtemann, "A luminescent solar concentrator with $7.1 \%$ power conversion efficiency," Phys. Stat. Sol., vol. 2, no. 6, pp. 257-9, 2008.

[11] F. Purcell-Milton and Y. Gun'ko, "Quantum dots for luminescent solar concentrators," J. Mater. Chem., vol. 22, pp. 16687-97, 2012.

[12] Z. Krumer, S. J. Pera, R. van Dijik-Moes, Y. Zhao, A. de Brouwer, E. Groeneveld, W. van Sark, R. Schropp, and C. de Mello Donegá, "Tackling self-absorption in luminescent solar concentrators with type-II colloidal quantum dots," Sol. Energ. Mat. Sol. Cells, vol. 111, pp. 57-65, 2013.

[13] C. S. Erickson, L. R. Bradshaw, S. Bradshaw, S. McDowall, J. D. Gilbertson, D. R. Gamelin, and D. L. Patrick, "Zero-reabsorption doped-nanocrystal luminescent solar concentrators," ACS Nano, vol. 8, no. 4, pp. 3461-7, 2014.

[14] N. D. Bronstein, L. Li, L. Xu, Y. Yao, V. E. Ferry, A. P. Alivisatos, and R. G. Nuzzo, "Luminescent solar concentration with semiconductor nanorods and transfer printed micro-silicon solar cells," ASC Nano, vol. 8, no. 1, pp. 44-53, 2013. 
[15] I. Coropceanu and M. G. Bawendi, "Core/shell quantum dot based luminescent solar concentrators with reduced reabsorption and enhanced efficiency," Nano Lett., vol. 14, pp. 4097-101, 2014.

[16] Sigma Aldrich. Lumidot CdSe/ZnS 640, core-shell type quantum dots, $5 \mathrm{mg} / \mathrm{ml}$ in toluene. [Online]. Available: http://www.sigmaaldrich.com/catalog/product/aldrich/ 694606?lang $=$ en\&region $=\mathrm{US}$

[17] C. Evans, L. Guo, J. Peterson, S. Maccagnano-Zacher, and T. Krauss, "Ultrabright PbSe magic-sized clusters," Nano Letters, vol. 8, no. 9, pp. 2896-9, 2008.

[18] D. K. G. de Boer, C. R. Ronda, W. Keur, and A. Meijerink, "New luminescent materials and filters for luminescent solar concentrators," Proc. of SPIE, vol. 8108, p. 81080E, 2011.

[19] M. G. Debije and P. P. C. Verbunt, "Thirty years of luminescent solar concentrator research: solar energy for the built environment," Adv. Energy Mater., vol. 2, pp. $12-35,2012$.

[20] T. Wang, J. Zhang, W. Ma, Y. Luo, L. Wang, Z. Hu, W. Wu, X. Wang, G. Zou, and Q. Zhang, "Luminescent solar concentrator employing rare earth complex with zero self-absorption loss," Solar Energy, vol. 85, pp. 2571-9, 2011.

[21] S. F. Daorta, A. Proto, R. Fusco, L. C. Andreani, and M. Liscidini, "Cascade luminescent solar concentrators," Appl. Phys. Lett., vol. 104, p. 153901, 2014.

[22] B. D. Markman, R. R. Ranade, and N. C. Giebink, "Nonimaging optics in luminescent solar concentration," Opt. Express, vol. 20, no. S5, pp. A622-9, 2012.

[23] T. Wang, B. Yu, B. Chen, Z. Hu, Y. Luo, G. Zou, and Q. Zhang, "A theoretical model of a cylindrical luminescent solar concentrator with a dye-doping coating," J. Opt., vol. 15, p. 055709, 2013.

[24] R. H. Inman, G. V. Shcherbatyuk, D. Medvedko, A. Gopinathan, and S. Ghosh, "Cylindrical luminescent solar concentrators with near-infrared quantum dots," Opt. Express, vol. 19, pp. 24308-13, 2011.

[25] E. Banaei and A. F. Abouraddy, "Fiber luminescent solar concentrator with 5.7\% conversion efficiency," Proc. of SPIE, vol. 8821, p. 882102, 2013.

[26] M. G. Debije, M. Van, P. P. C. Verbunt, M. J. Kastelijn, R. H. L. van der Blom, D. J. Broer, and C. W. M. Bastiaansen, "Effect on the output of a luminescent solar concentrator on application of organic wavelength-selective mirrors," Appl. Optics, vol. 49, no. 4, pp. 745-51, 2010.

[27] C. Corrado, S. W. Leow, M. Osborn, E. Chan, B. Balaban, and S. A. Carter, "Optimization of gain and energy conversion efficiency using front-facing photovoltaic cell luminescent solar concentrator design," Sol. Energ. Mat. Sol. Cells, vol. 111, pp. 74-81, 2013.

[28] L. Desmet, A. J. M. Ras, D. K. G. de Boer, and M. G. Debije, "Monocrystalline silicon photovoltaic luminescent solar concentrator with $4.2 \%$ power conversion efficiency," Opt. Lett., vol. 37, no. 15, pp. 3087-89, 2012. 
[29] J. Bomm, A. Büchtemann, A. J. Chatten, R. Bose, D. J. Farell, N. L. A. Chan, Y. Xiao, L. H. Slooff, T. Meyer, A. Meyer, W. van Sark, and R. Koole, "Fabrication and full characterization of state-of-the-art quantum dot luminescent solar concentrators," Sol. Energ. Mat. Sol. Cells, vol. 95, pp. 2087-94, 2011.

[30] G. V. Shcherbatyuk, R. H. Inman, C. Wang, R. Winston, and S. Ghosh, "Viability of using near infrared $\mathrm{PbS}$ quantum dots as active materials in luminescent solar concentrators," Appl. Phys. Lett., vol. 96, p. 191901, 2010.

[31] A. Bueno, I. Suárez, R. Abargues, S. Sales, and J. P. M. Pastor, "Temperature sensor based on colloidal quantum dots-PMMA nanocomposite waveguides," IEEE Sensors J., vol. 12, no. 10, pp. 3069-74, 2012.

[32] R. Tycko, "Remote sensing of sample temperatures in nuclear magnetic resonance using photoluminescence of semiconductor quantum dots," J. Magn. Reson., vol. 244, pp. 64-7, 2014.

[33] H. Sakaue, A. Aikawa, Y. Iijima, T. Kuriki, and T. Miyazaki, Quantum dots-a variety of new applications, A. Al-Ahmadi, Ed. InTech, 2004. [Online]. Available: http://www.intechopen.com/books/quantum-dots-a-variety-of-new-applications

[34] F. Vetrone, R. Naccache, A. Zamarrón, A. J. de la Fuente, F. Sanz-Rodríguez, L. M. Maestro, E. M. Rodriguez, D. Jaque, J. Solé, and J. A. Capobianco, "Temperature sensing using fluorescent nanothermometers," ASC Nano, vol. 7, pp. 3254-8, 2010.

[35] S. Li, K. Zhang, J. Yang, L. Lin, and H. Yang, "Single quantum dots as local temperature markers," Nano Lett., vol. 7, no. 10, pp. 3102-5, 2007.

[36] M. A. Carpenter and M. A. Petrukhina, "Development of a portable petroleum byproducts chemical sensor: Phase III and IV," University at Albany, SUNY, Tech. Rep., 2009. [Online]. Available: https://www.dot.ny.gov/divisions/engineering/technicalservices/trans-r-and-d-repository/C-02-08_Final\%20Report_Phase\%203-4.pdf

[37] H. Liu, M. Li, O. Voznyy, L. Hu, Q. Fu, D. Zhou, Z. Xia, and E. H. S. J. Tang, "Physically flexible, rapid-response gas sensor based on colloidal quantum dot solids," Adv. Mater., vol. 26, pp. 2718-24, 2014.

[38] C. L. Wang, L. Gou, J. M. Zaleski, and D. L. Friesel, "ZnS quantum dot based nanocomposite scintillators for thermal neutron detection," Nucl. Instrum. Meth., vol. 622, pp. 186-90, 2010.

[39] W. G. Lawrence, S. Thacker, S. Palamakumbura, K. J. Riley, and V. V. Nagarkar, "Quantum dot-organic polymer composite materials for radiation detection and imaging," Trans. Nucl. Sci., vol. 59, no. 1, pp. 215-21, 2012.

[40] I. H. Campbell and B. K. Crone, "Quantum-dot/ organic semiconductor composites for radiation detection," Adv. Mater., vol. 18, pp. 77-79, 2006.

[41] S. Wei, J. Sampathi, Z. Guo, N. Anumandla, D. Rutman, A. Kucknoor, L. James, and A. Wang, "Nanoporous poly(methyl methacrylate)-quantum dots nanocomposite fibers towards biomedical applications," Polymer, vol. 52, pp. 5817-29, 2011. 
[42] W. Chung, K. Park, H. J. Yu, J. Kim, B. Chun, and S. H. Kim, "White emission using mixtures of CdSe quantum dots and PMMA as a phosphor," Opt. Mat., vol. 32, pp. 515-21, 2010.

[43] N. Shah et al., "Halving global CO2 by 2050: technologies and costs," Imperial College London, Technical Report, 2013. [Online]. Available: https:// workspace.imperial.ac.uk/climatechange/Public/pdfs/Collaborative\%20publications/ Halving\%20global\%20CO2\%20by\%202050\%20-\%20technologies\%20and\%20costs\%20\%20Imperial\%20College\%20London.pdf

[44] A. Goetzberger, J. Knobloch, and B. Voss, Crystalline Silicon Solar Cells. John Wiley \& Sons, Ltd, 1998.

[45] A. Goetzberger, C. Hebling, and H. W. Schock, "Photovoltaic materials, history, status and outlook," Materials Science and Engineering R, vol. 40, pp. 1-46, 2003.

[46] R. W. Miles, K. M. Hynes, and I. Forbes, "Photovoltaic solar cells: an overview of state-of-the-art development and environmental issues," Progress in Crystal Growth and Characterization of Materials, vol. 51, pp. 1-42, 2005.

[47] M. A. Green, K. Emery, Y. Hishikawa, W. Warta, and E. D. Dunlop, "Solar cell efficiency tables (version 45)," Prog. Photovolt: Res. Appl., vol. 23, pp. 1-9, 2015.

[48] D. Neamen, An Introduction to Semiconductor Devices, 1st ed. McMgraw-Hill, 2006.

[49] A. I. Ekimov and A. A. Onushchenko, Pis'ma Zh. Eksp. Teor. Fiz., vol. 34, no. 6, pp. 363-6, 1981.

[50] A. I. Ekimov, A. L. Efros, and A. A. Onushchenko, "Quantum size effect in semiconductor microcrystals," Solid State Communications, vol. 56, no. 11, pp. 921-4, 1985.

[51] L. E. Brus, "Electron-electron and electron-hole interactions in small semiconductor crystallites: the size dependence of the lowest excited electronic state," J. Chem. Phys., vol. 80, no. 9, pp. 4403-9, 1984.

[52] L. E. Brus, "A simple model for the ionization potential, electron affinity, and aqueous redox potentials of small semiconductor crystallites," J. Chem. Phys., vol. 79, no. 11, pp. 5566-71, 1983.

[53] A. Surrente, P. Gallo, M. Felici, B. Dwir, A. Rudra, and E. Kapon, "Dense arrays of ordered pyramidal quantum dots with narrow linewidth photoluminescence spectra," Nanotechnology, vol. 20, p. 415205, 2009.

[54] L. K. Lee, L. Zhang, H. Deng, and P. C. Ku, "Room-temperature quantum-dot-like luminescence from site-controlled InGaN quantum disks," Appl. Phys. Lett., vol. 99, p. 263105, 2011.

[55] L. Manna, E. C. Scher, and A. P. Alivisatos, "Synthesis of soluble and processable rod-, arrow-, teardrop-, and tetrapod-shaped CdSe nanocrystals," J. Am. Chem. Soc., vol. 122, pp. $12700-6,2000$.

[56] M. S. Wong. (2013) CdSe quantum dots. Adapted from original work, CC BY-SA 3.0 license. [Online]. Available: http://commons.wikimedia.org/wiki/File: CdSe_Quantum_Dots.jpg\# 
[57] B. O. Dabbousi, J. Rodriguez-Viejo, F. V. Mikulec, J. R. Heine, H. Mattoussi, R. Ober, K. F. Jensen, and M. G. Bawendi, "(CdSe)ZnS core-shell quantum dots: synthesis and characterization of a size series of highly luminescent nanocrystallites," J. Phys. Chem. $B$, vol. 101, pp. 9463-75, 1997.

[58] P. Reiss, M. Protière, and L. Li, "Core/shell semiconductor nanocrystals," Small, vol. 5, no. 2, pp. 154-68, 2009.

[59] D. V. Talapin, R. Koeppe, S. Götzinger, A. Kornowski, J. M. Lupton, A. L. Rogach, O. Benson, J. Feldmann, and H. Weller, "Highly emissive colloidal CdSe/CdS heterostructures of mixed dimensionality," Nano Lett., vol. 3, no. 12, pp. 1677-81, 2003.

[60] F. Meinardi, A. Colombo, K. A. Velizhanin, R. Simonutti, M. Lorenzon, L. Beverina, R. Viswanatha, V. I. Klimov, and S. Brovelli, "Large-area luminescent solar concentrators based on 'Stokes-shift-engineered' nanocrystals in a mass-polymerized PMMA matrix," Nature Photonics, vol. 8, pp. 392-9, 2014.

[61] S. Kim, B. Fisher, H. J. Eisler, and M. Bawendi, "Type-II quantum dots: $\mathrm{CdTe} / \mathrm{CdSe}($ core/shell) and CdSe/ZnTe(core/shell) heterostructures," J. Am. Chem. Soc., vol. 125, pp. $11466-7,2003$.

[62] P. Sarkar, M. Springborg, and G. Seifert, "A theoretical study of the structural and electronic properties of CdSe/CdS and CdS/CdSe core/shell nanoparticles," Chem. Phys. Lett., vol. 405, pp. 103-7, 2004.

[63] D. V. Talapin and C. B. Murray, "PbSe nanocrystal solids for n- and p-channel thin film field-effect transistors," Science, vol. 310, pp. 86-9, 2005.

[64] H. Zhu, M. Z. Hu, L. Shao, R. Dabestani, M. B. Zaman, and S. Liao, "Synthesis and optical properties of thiol functionalized CdSe/ZnS (core/shell) quantum dots by ligand exchange," Journal of Nanomaterials, vol. 2014, pp. 1-14, 2014.

[65] D. A. Hines and P. V. Kamat, "Quantum dot surface chemistry: ligand effects and electron transfer reactions," J Phys. Chem. C, vol. 117, pp. 14418-26, 2013.

[66] K. Kumari, U. Kumar, S. N. Sharma, S. Chand, R. Kakkar, V. D. Vankar, and V. Kumar, "Effect of surface passivating ligand on structural and optoelectronic properties of polymer: CdSe quantum dot composites," J Phys. D: Appl. Phys., vol. 41, p. 235409, 2008.

[67] C. Radhakrishnan, M. K. F. Lo, C. M. Knobler, M. A. Garcia-Garibay, and H. G. Monbouquette, "Capping-ligand effect on the stability of CdSe quantum dot langmuir monolayers," Langmuir, vol. 27, pp. 2099-103, 2011.

[68] A. M. Dennis, D. C. Sotto, B. C. Mei, I. L. Medintz, H. Mattoussi, and G. Bao, "Surface ligand effects on metal-affinity coordination to quantum dots: Implications for nanoprobe self-assembly," Bioconjugate Chem., vol. 21, pp. 1160-70, 2010.

[69] W. C. W. Chan and S. Nie, "Quantum dot bioconjugates for ultrasensitive nonisotropic detection," Science, vol. 281, pp. 2016-8, 1998.

[70] B. L. Anderson and R. L. Anderson, Fundamentals of Semiconductor Devices. McGraw Hill, 2005. 
[71] M. Fox, Optical Properties of Solids. Oxford University Press, 2007.

[72] F. W. Wise, "Lead salt quantum dots: the limit of strong quantum confinement," Acc. Chem. Res., vol. 33, pp. 773-780, 2000.

[73] J. G. Solé, L. E. Bausá, and D. Jaque, An Introduction to the Optical Spectroscopy of Inorganic Solids. John Wiley \& Sons, Ltd, 2005.

[74] D. J. Norris, Nanocrystal Quantum Dots, 2nd ed., V. Klimov, Ed. CRC Press, 2010.

[75] M. T. Trinh, "Carrier multiplication and exciton behavior in PbSe quantum dots," Doctoral Thesis, Technische Universiteit Delft, 2010. [Online]. Available: http://repository.tudelft.nl/view/ir/uuid\%3Af50bcf2a-97ec-4c39-99fd-5b09349256ae/

[76] P. D. Fairley, "Novel solid state modulator for the infrared: the germanium chopper," Doctoral Thesis, University of Southhampton, 2000. [Online]. Available: http://www.orc.soton.ac.uk/viewpublication.html?pid=1420T

[77] A. J. Houtepen, "Charge injection and transport in quantum confined and disordered systems," Doctoral Thesis, Universiteit Utrecht, 2007. [Online]. Available: http://dspace.library.uu.nl/handle/1874/21793

[78] M. Kasha, "Characterization of electronic transitions in complex molecules," Discuss. Faraday Soc., vol. 9, pp. 14-9, 1950.

[79] B. Herman, J. R. Lakowicz, D. B. Murphy, T. J. Fellers, and M. W. Davidson. (2009) Fluorescence excitation and emission fundamentals. [Online]. Available: http://www.olympusconfocal.com/theory/fluoroexciteemit.html

[80] T. Förster, "10th Spiers memorial lecture: transfer mechanisms of electronic excitation," Discussions of the Faraday Society, vol. 27, pp. 7-17, 1959.

[81] T. Förster, "Transfer mechanisms of electronic excitation energy," Radiation Research Supplement, vol. 2, pp. 326-39, 1960.

[82] D. L. Andrews, Tutorials in Complex Photonic Media, M. Noginov, G. Dewar, M. McCall, and N. Zheludev, Eds. SPIE, 2009. [Online]. Available: http://spie.org/samples/pm194.pdf

[83] A. J. Mork, M. C. Weidman, F. Prins, and W. A. Tisdale, "Magnitude of the Förster radius in colloidal quantum dots," J. Phys. Chem. C, vol. 118, pp. 13920-8, 2014.

[84] T. Kippeny, L. A. Swafford, and S. J. Rosenthal, "Semiconductor nanocrystals: a powerful visual aid for introducing the particle in a box," J. Chem. Educ., vol. 79, no. 9, pp. 1094-100, 2002.

[85] H. Preier, "Recent advances in lead-chalcogenide diode lasers," Appl. Phys., vol. 20, pp. 189-206, 1979.

[86] P. Vachaspati, "Quantum dots: Theory, application, synthesis," 2013. [Online]. Available: http://pranjalv.com/qdots.pdf

[87] D. L. Waldron, A. Preske, J. M. Zawodny, T. D. Krauss, and M. C. Gupta, "Lead selenide quantum dot polymer nanocomposites," Nanotechnology, vol. 26, no. 7, p. $075705,2015$. 
[88] A. K. Walton, T. S. Moss, and B. Ellis, "Determination of the electron effective mass in the lead salts by the infra-red Faraday effect," Proc. Phys. Soc., vol. 79, pp. 1065-8, 1962.

[89] I. Kang and F. W. Wise, "Electronic structure and optical properties of PbS and PbSe quantum dots," J. Opt. Soc. Am. B, vol. 14, no. 7, pp. 1632-46, 1997.

[90] N. M. Ravindra and V. K. Srivastava, "Properties of PbS, PbSe, and PbTe," Phys. Stat. Sol. (a), vol. 58, no. 1, pp. 311-6, 1980.

[91] "Sigma Aldrich solvent center: hexane." [Online]. Available: http://www.sigmaaldrich. com/chemistry/solvents/hexane-center.html

[92] Y. Varshni, "Temperature dependence of the energy gap in semiconductors," Physica, vol. 34, pp. 149-54, 1967.

[93] D. Pugh-Thomas, B. Walsh, and M. C. Gupta, "CdSe(ZnS) nanocomposite luminescent high temperature sensor," Nanotechnology, vol. 22, 2011.

[94] D. Olego and M. Cardona, "Photoluminescence in heavily doped GaAs," Phys. Rev. B, vol. 22, no. 2, pp. 886-93, 1980.

[95] F. G. Sánchez-Almazan, H. Navarro-Contreras, G. Ramírez-Flores, M. A. Vidal, O. Zelaya-Angel, M. E. Rodríguez, and R. Baquero, "Temperature dependence of the band gap of $\mathrm{Cd}_{1-x} \mathrm{Zn}_{x}$ Te alloys of low zinc concentrations," J. Appl. Phys., vol. 79, pp. 7713-17, 1996.

[96] Y. I. Ravich, Semiconducting Lead Chalcogenides. Springer Science \& Business Media, 2013.

[97] A. Olkhovets, R. C. Hsu, A. Lipovskii, and F. W. Wise, "Size-dependent temperature variation of the energy gap in lead-salt quantum dots," Phys. Rev. Lett., vol. 81, p. 3539, 1998.

[98] T. J. Liptay and R. Ram, "Temperature dependence of the exciton transition in semiconductor quantum dots," Appl. Phys. Lett., vol. 89, p. 223132, 2006.

[99] J. E. Boercker, E. M. Clifton, J. G. Tischler, E. E. Foos, T. J. Zega, M. E. Twigg, and R. M. Stroud, "Size and temperature dependence of band-edge excitons in PbSe nanowires," J. Phys. Chem. Lett., vol. 2, pp. 527-31, 2011.

[100] O. E. Semonin, J. C. Johnson, J. M. Luther, A. G. Midgett, A. J. Nozik, and M. C. Beard, "Absolute photoluminescence quantum yields of IR-26 dye, PbS, and PbSe quantum dots," J. Phys. Chem. Lett., vol. 1, pp. 2445-50, 2010.

[101] E. Lifshitz, M. Brumer, A. Kigel, A. Sashchiuk, M. Bashouti, M. Sirota, E. Galun, Z. Burshtein, A. Q. Le Quang, I. Ledoux-Rak, and J. Zyss, "Air-stable PbSe/PbS and $\mathrm{PbSe} / \mathrm{PbSe}_{x} \mathrm{~S}_{1-x}$ core-shell nanocrystal quantum dots and their applications," J. Phys. Chem. B, vol. 110, pp. 25 356-65, 2006.

[102] J. Lee, E. S. Koteles, and M. O. Vassekk, "Luminescence linewidths of excitons in GaAs quantum wells below 150K," Phys. Rev. B, vol. 33, no. 8, pp. 5512-16, 1986.

[103] L. G. Zhang, D. Z. Shen, X. W. Fan, and S. Z. Lu, "Exciton-phonon scattering in CdSe/ZnSe quantum dots," Chin. Phys. Lett., vol. 19, no. 4, pp. 578-80, 2002. 
[104] A. Kigel, M. Brumer, G. I. Maikov, A. Sashchiuk, and E. Lifshitz, "Thermally activated photoluminescence in lead selenide colloidal quantum dots," Small, vol. 5, no. 14, pp. 1675-81, 2009.

[105] B. Henderson and G. F. Imbush, Optical Spectroscopy of Inorganic Solids. Oxford University Press, 1989.

[106] Y. Zhao, C. Riemersma, F. Pietra, R. Koole, and A. Meijerink, "High-temperature luminescence quenching of colloidal quantum dots," ACS Nano, vol. 6, no. 10, pp. 9058-67, 2012.

[107] D. Valerini, A. Cretí, and M. Lomascolo, "Temperature dependence of the photoluminescence properties of colloidal CdSe/ZnS core/shell quantum dots embedded in a polystyrene matrix," Physical Review B, vol. 71, p. 235409, 2005.

[108] G. Morello, M. De Giorgi, S. Kudera, L. Manna, R. Cingolani, and M. Anni, "Temperature and size dependence of nonradiative relaxation and exciton-phonon coupling in colloidal CdTe quantum dots," J. Phys. Chem. C, vol. 111, pp. 5846-5849, 2007.

[109] H. Wu, Y. Zhang, L. Yan, Y. Jiang, T. Zhang, Y. Feng, H. Chu, Y. Wang, J. Zhao, and W. W. Yu, "Temperature effect on colloidal PbSe quantum dot-filled liquid-core optical fiber," Optical Materials Express, vol. 4, no. 9, pp. 1856-65, 2014.

[110] S. A. Empedocles, D. J. Norris, and M. G. Bawendi, "Photoluminescence spectroscopy of single CdSe nanocrystallite quantum dots," Physical Review Letters, vol. 77, no. 18, pp. 3873-6, 1996.

[111] S. V. Kilina, D. S. Kilin, V. V. Prezhdo, and O. V. Prezhdo, "Theoretical study of electron-phonon relaxation in PbSe and CdSe quantum dots: evidence for phonon memory," J. Phys. Chem. C, vol. 115, pp. 21641-51, 2011.

[112] H. Wang, A. Yang, and C. Sui, "Luminescent high temperature sensor based on the CdSe/ZnS quantum dot thin film," Optoelecton. Lett., vol. 9, no. 6, pp. 421-4, 2013.

[113] Y. Zhao, C. Riemersma, F. Pietra, R. Koole, C. de Mello Donegá, and A. Meijerink, "Temperature dependence of the energy gap in semiconductors," ACS Nano, vol. 6, no. 10, pp. 9058-67, 2012.

[114] A. L. Efros, V. A. Kharchenko, and M. Rosen, "Breaking the phonon bottleneck in nanometer quantum dots: role of Auger-like processes," Solid State Communications, vol. 93, pp. 281-84, 1995.

[115] R. R. Cooney, S. L. Sewall, E. A. Dias, D. M. Sagar, K. E. H. Anderson, and P. Kambhampati, "Unified picture of electron and hole relaxation pathways in semiconductor quantum dots," Physical Review B, vol. 75, p. 245311, 2007.

[116] P. Bendt and A. Rabl, "Optical analysis of point focus parabolic radiation concentrators," Appl. Opt., vol. 20, no. 4, pp. 674-83, 1981.

[117] A. Rabl, "Solar concentrators with maximal concentration for cylindrical absorbers," Appl. Opt., vol. 15, no. 7, pp. 1871-3, 1976. 
[118] N. Fraidenraich, C. Tiba, B. B. Brandão, and O. Vilela, "Analytic solutions for the geometric and optical properties of stationary compound parabolic concentrators with fully illuminated inverted V receiver," Solar Energy, vol. 82, pp. 132-143, 2008.

[119] D. Jing, H. Liu, X. Zhang, L. Zhao, and L. Guo, "Photocatalytic hydrogen production under direct solar light in a CPC based solar reactor: reactor design and preliminary results," Energy Conversion and Management, vol. 50, pp. 2919-26, 2009.

[120] N. Yeh, "Analysis of spectrum distribution and optical losses under fresnel lenses," Renewable and Sustainable Energy Reviews, vol. 14, pp. 2926-35, 2010.

[121] K. Ryu, J. Rhee, K. Park, and J. Kim, "Concept and design of modular fresnel lenses for concentration solar PV system," Solar Energy, vol. 80, pp. 1580-87, 2006.

[122] Y. Tripanagnostopoulos, C. Siabekou, and J. K. Tonui, "The Fresnel lens concept for solar control of buildings," Solar Energy, vol. 81, pp. 661-75, 2007.

[123] S. Hatwaambo, H. Hakansson, A. Roos, and B. Karlsson, "Mitigating the non-uniform illumination in low concentrating CPCs using structured reflectors," Sol. Energ. Mat. Sol. Cells, vol. 93, pp. 2020-4, 2009.

[124] P. A. Davies, "Light-trapping lenses for solar cells," Appl. Opt., vol. 31, no. 28, pp. 6021-6, 1992.

[125] National Renewable Energy Laboratory. (2013) Concentrating solar power projects. [Online]. Available: http://www.nrel.gov/csp/solarpaces/by_country_detail.cfm/ country=US

[126] R. Winston, "Light collection within the framework of geometrical optics," J. Opt. Soc. Am, vol. 60, no. 2, pp. 245-47, 1970.

[127] G. Smestad, H. Ries, R. Winston, and E. Yablonovich, "The thermodynamic limits of light concentrators," Solar Energy Materials, vol. 21, pp. 99-111, 1990.

[128] A. Rabl, "Comparison of solar concentrators," Solar Energy, vol. 18, no. 2, pp. 93-111, 1976.

[129] W. A. Shurcliff and R. C. Jones, "The trapping of fluorescent light produced within objects of high geometrical symmetry," J. Opt. Soc. Am., vol. 39, no. 11, pp. 912-6, 1949.

[130] G. Keil, "Design principles of fluorescence radiation converters," Nuclear Instruments and Methods, vol. 87, pp. 111-23, 1970.

[131] W. R. L. Thomas, J. M. Drake, and M. L. Lesiecki, "Light transport in planar luminescent solar concentrators: the role of matrix losses," Appl. Opt., vol. 22, no. 21, pp. 3440-50, 1983.

[132] T. Kaino, "Absorption losses of low loss plastic optical fibers," Jpn. J. Appl. Phys., vol. 24, pp. 1661-5, 1985.

[133] P. Debye and A. M. Bueche, "Scattering by an inhomogeneous solid," J. Appl. Phys., vol. 20, pp. 518-25, 1949. 
[134] 3M Corp. (2010) Vikuiti enhanced specular reflector (ESR). [Online]. Available: http://multimedia.3m.com/mws/media/374730O/vikuiti-tm-esr-salesliterature.pdf?fn=ESR $\% 20$ ss2.pdf

[135] M. F. Waber, C. A. Stover, L. R. Gilbert, T. J. Nevitt, and A. J. Ouderkirk, "Giant birefringent optics in multilayer polymer mirrors," Science, vol. 287, pp. 2451-6, 2000.

[136] E. Yablonovitch, "Thermodynamics of the fluorescent plate concentrator," J. Opt. Soc. Am., vol. 70, no. 11, pp. 1362-3, 1980.

[137] W. L. Rasmussen, "Novel carbazole based methacrylates, acrylates, and dimethacrylates to produce high refractive index polymers," Doctoral Thesis, Virginia Tech, 2001. [Online]. Available: http://scholar.lib.vt.edu/theses/available/etd-12202001-135708/

[138] L. J. Mathias. (2005) Polymer science learning center: free radical vinyl polymerization. [Online]. Available: http://pslc.ws/macrog/radical.htm

[139] W. Rutsch, K. Dietliker, D. Leppard, M. Köhler, L. Misev, U. Kolczak, and G. Rist, "Recent developments in photoinitiators," Progress in Organic Coatings, vol. 27, pp. 227-39, 1996.

[140] U. Kolczak, G. Rist, K. Dietliker, and J. Wirz, "Reaction mechanism of monacyl- and biacylphosphine oxide photoinitiators studied by ${ }^{31} \mathrm{P}-,{ }^{13} \mathrm{C}-$, and ${ }^{1} \mathrm{H}-\mathrm{CIDNP}$ and ESR," J. Am. Chem. Soc., vol. 118, pp. 6477-89, 1996.

[141] W. P. Mulligan, D. H. Rose, M. J. Cudzinovic, D. M. De Ceuster, K. R. McIntosh, D. D. Smith, and R. M. Swanson, "Manufacture of solar cells with 21\% efficiency," EU PVSEC Conference Proceedings, 2004. [Online]. Available: http://tayloredge.com/reference/Electronics/Photonics/HighEfficiencySolarCells.pdf

[142] G. Vuye, S. Fisson, V. N. Van, Y. Wang, J. Rivory, and F. Abelès, "Temperature dependence of the dielectric function of silicon using in situ spectroscopic ellipsometry," Thin Solid Films, vol. 233, pp. 166-70, 1993. [Online]. Available: http://refractiveindex.info/?shelf=main\&book=Si\&page=Vuye-20C

[143] H. H. Li, "Refractive index of silicon and germanium and its wavelength and temperature derivatives," J. Phys. Chem. Ref. Data, vol. 9, no. 3, pp. 561-658, 1980. [Online]. Available: http://refractiveindex.info/?shelf=main\&book=Si\&page=Li-293K

[144] L. Gao, F. Lemarchand, and M. Lequime, "Exploitation of multiple incidences spectrometric measurements for thin film reverse engineering," Optics Express, vol. 20, no. 14, pp. 15734-51, 2012. [Online]. Available: http://refractiveindex.info/?shelf=main\&book=SiO2\&page=Lemarchand

[145] L. Gao, F. Lemarchand, and M. Lequime, "Refractive index determination of SiO2 layer in the UV/Vis/NIR range: spectrophotometric reverse engineering on single and bi-layer designs," J. Europ. Opt. Soc. Rap. Public, vol. 8, p. 13010, 2013. [Online]. Available: http://refractiveindex.info/?shelf $=$ main\&book $=\mathrm{SiO} 2 \&$ page $=\mathrm{Gao}$

[146] H. R. Philipp, "Optical properties of silicon nitride," J. Electrochem. Soc. Solid-State Science and Technology, vol. 120, no. 2, pp. 295-300, 1973. [Online]. Available: http://refractiveindex.info/?shelf=main\&book=Si3N4\&page=Philipp 
[147] N. Sultanova, S. Kasarova, and I. Nikolov, "Dispersion properties of optical polymers," Acta Physica Polonica A, vol. 116, no. 4, pp. 585-7, 2009. [Online]. Available: http://refractiveindex.info/?shelf=organic\&book=poly\%28methyl_methacrylate\% 29\&page $=$ Sultanova

[148] Fiber Optic Center, Inc. (2000) AngstromBond AB9093 datasheet. [Online]. Available: http://www.focenter.com/Fiber\%20Optic\%20Center\%5Cangstrombond $\% 5$ Cdata\% 20sheets\%5C9093.pdf

[149] Sigma-Aldrich, Inc. (2015) Lauryl methacrylate. [Online]. Available: https: //www.sigmaaldrich.com/catalog/product/aldrich/291811?lang=en\&region=US

[150] Scientific Polymer, Inc. (2013) Refractive index of polymers by index. [Online]. Available: http://scientificpolymer.com/technical-library/refractive-index-of-polymersby-index/

[151] BASF, Inc. (2012) Lauryl methacrylate 1214. [Online]. Available: http://www.specialtymonomers.basf.com/portal/streamer?fid=235699

[152] The Dow Chemical Company. (2015) Rocryl 320 lauryl methacrylate (LMA). [Online]. Available: http://www.dow.com/products/market/construction/product-line/rocrylalkyl-esters/product/rocryl-320-lauryl-methacrylate-\%28lma\%29/

[153] Sigma-Aldrich, Inc. (2015) Ethylene glycol dimethacrylate. [Online]. Available: http://www.sigmaaldrich.com/catalog/product/aldrich/335681?lang=en\&region=US

[154] ChemicalBook. (2010) Poly(ethylene glycol) dimethacrylate. [Online]. Available: http://www.chemicalbook.com/ChemicalProductProperty_EN_CB4344535.htm

[155] The Dow Chemical Company. (2011) Ethylene glycol dimethacrylate (EGDMA). [Online]. Available: http://www.specialty-monomers.basf.com/portal/streamer?fid= 235731

[156] W. Jarosz, "Efficient monte carlo methods for light transport in scattering media," Doctoral Thesis, UC San Diego, 2008. [Online]. Available: http: //www.cs.dartmouth.edu/ wjarosz/publications/dissertation/

[157] A. Laughney. (2009) A monte carlo model of light propagation in tissue. [Online]. Available: http://engineering.dartmouth.edu/ d25559k/ENGG168_files/ Notes/MC_LightPropagationApp.pptx

[158] A. Schüler, A. Kostro, B. Huriet, C. Galande, and J. L. Scartezzini, "Monte carlo simulations of quantum dot solar concentrators: ray tracing based on fluorescence mapping," Proc. of SPIE, vol. 7046, p. 704609, 2008.

[159] H. H. Barrett and K. J. Myers, Foundations of Image Science. John Wiley \& Sons, Ltd, 2004.

[160] D. Şahin, B. Ilan, and D. F. Kelley, "Monte-carlo simulations of light propagation in luminescent solar concentrators," J. Appl. Phys., vol. 110, p. 033108, 2011.

[161] M. Chelle, "Could plant leaves be treated as lambertian surfaces in dense crop canopies to estimate light absorption?" Ecological Modeling, vol. 198, pp. 219-28, 2006. 
[162] J. Junesch, T. Sannomiya, and A. B. Dahlin, "Optical properties of nanohole arrays in metal-dielectric double films prepared by mask-on-metal colloidal lithography," ACS Nano, vol. 6, no. 11, pp. 10405-10415, 2012. [Online]. Available: http://adahlin.com/matlab.html

[163] C. C. Katsidis and D. I. Siapkas, "General transfer-matrix method for optical multilayer systems with coherent, partially coherent, and incoherent interference," Applied Optics, vol. 41, no. 19, pp. 3978-87, 2002.

[164] A. Goetzberger, J. Knobloch, and B. Voss, Crystalline Silicon Solar Cells. John Wiley \& Sons, Ltd, 1998.

[165] W. G. J. H. M. van Sark, "Luminescent solar concentrators - a low cost photovoltaic alternative," EPJ Web of Conferences, vol. 33, p. 02003, 2012.

[166] W. G. J. H. M. van Sark, K. W. J. Barnham, L. H. Slooff, A. J. Chatten, A. Büchtemann, A. Meyer, S. J. McCormack, R. Koole, D. J. Farrell, R. Bose, E. E. Bende, A. R. Burgers, T. Budel, J. Quilitz, M. Kennedy, T. Meyer, C. de Mello Donegá, A. Meijerink, and D. Vanmaekelbergh, "Luminescent solar concentrators: a review of recent results," Optics Express, vol. 16, no. 26, pp. 21773-92, 2008.

[167] L. Pan, Y. Shen, K. Tetz, and Y. Fainman, "PMMA quantum dots composites fabricated via use of pre-polymerization," Optics Express, vol. 13, no. 1, pp. 44-9, 2005.

[168] C. M. Evans, M. E. Evans, and T. D. Krauss, "Mysteries of TOPSe revealed: Insights into quantum dot nucleation," J. Am. Chem. Soc., vol. 132, no. 32, pp. $10973-75$, 2010.

[169] Q. Dai, Y. Wang, X. Li, D. Pellegrino, M. Zhao, B. Zou, J. Seo, Y. Wang, and W. Yu, "Size-dependent composition and molar extinction coefficient of PbSe semiconductor nanocrystals," ACS Nano, vol. 3, no. 6, pp. 1518-24, 2009.

[170] I. Moreels, K. Lambert, D. De Muynck, F. Vanhaecke, D. Poelman, J. Martins, G. Allan, and Z. Hens, "Composition and size-dependent extinction coefficient of colloidal PbSe quantum dots," Chem. Mater., vol. 19, pp. 6101-6, 2007.

[171] J. Jasieniak, J. Pacifico, R. Signorini, A. Chiasera, M. Ferrari, A. Martucci, and P. Mulvaney, "Luminescence and amplified stimulated emission in CdSe-ZnSnanocrystal-doped TiO2 and $\mathrm{ZrO} 2$ waveguides," Adv. Funct. Mater., vol. 17, pp. 1654-62, 2007.

[172] Smooth-On Inc, "Crystal clear series clear urethane casting resins." [Online]. Available: http://www.smooth-on.com/tb/files/CRYSTAL_CLEAR_200_TB.pdf

[173] EpoxySet Inc, "Technical bulletin: Epoxibond-107LP-2." [Online]. Available: http://www.epoxysetinc.com/wp-content/themes/twentytwelve/opticalAdhesivespdf/EB-107LP-2.pdf

[174] D. L. Waldron, A. Preske, J. M. Zawodny, T. Krauss, and M. C. Gupta, "PbSe quantum dot based luminescent solar concentrators," 2015, manuscript under preparation.

[175] T. Ahn, R. Al-Kaysi, A. Müller, K. Wentz, and C. Bardeen, "Self-absorption correction for solid-state photoluminescence quantum yields obtained from integrating sphere measurements," Rev. Sci. Instrum., vol. 78, no. 8, p. 086105, 2007. 
[176] I. M. Smallwood, Solvent Recovery Handbook. CRC Press, LLC, 2002.

[177] J. Franc, D. Blanc, A. Zerroukhi, Y. Chalamet, A. Last, and N. Destouches, "Organosilicatitania nanocomposite elaborated by solgel processing with tunable optical properties," Materials Science and Engineering: B, vol. 129, pp. 180-5, 2006.

[178] B. Rowan, "The development of a quantum dot solar concentrator," Doctoral Thesis, Dublin Institute of Technology, 2007. [Online]. Available: http://arrow.dit.ie/cgi/viewcontent.cgi?article $=1012 \&$ context $=$ sciendoc

[179] "Optical constants of PbSe." [Online]. Available: http://refractiveindex.info/legacy/ ?group $=$ CRYSTALS\&material $=$ PbSe\&option $=$ sopra

[180] "Sigma Aldrich solvent center: toluene." [Online]. Available: http: //www.sigmaaldrich.com/chemistry/solvents/toluene-center.html

[181] B. Siroka, "Approximate dielectric constant of AB9093 epoxy," 2014, personal correspondence.

[182] M. Sauer, J. Hofkens, and J. Enderlein, Handbook of Fluorescence Spectroscopy and Imaging. Wiley-VCH, 2011. [Online]. Available: http://www.wileyvch.de/books/sample/3527316698_c01.pdf

[183] R. Liand, R. Tian, W. Shi, Z. Liu, D. Yan, M. Wei, D. G. Evans, and X. Duan, “A temperature sensor based on CdTe quantum dots-layered double hydroxide ultrathin films via layer-by-layer assembly," Chem. Comm., vol. 49, no. 10, pp. 933-1024, 2013.

[184] B. Larrión, M. Hernáez, F. J. Arregui, J. Goicoechea, J. Bravo, and I. R. Matías, "Photonic crystal fiber temperature sensor based on quantum dot nanocoatings," Journal of Sensors, vol. 2009, 2009.

[185] P. Singh and N. M. Ravindra, "Temperature dependence of solar cell performance - an analysis," Sol. Energ. Mat. Sol. Cells, vol. 101, pp. 36-45, 2012.

[186] Sunpower Corporation, "E19/ 320 solar panel." [Online]. Available: http: //us.sunpower.com/sites/sunpower/files/media-library/data-sheets/ds-e19-series-320solar-panel-datasheet.pdf

[187] K. Sun, Apr. 2015, personal correspondence.

[188] J. W. E. Wiegman and E. van der Kolk, "Building integrated thin film luminescent solar concentrators: Detailed efficiency characterization and light transport modelling," Solar Energy Materials and Solar Cells, vol. 103, pp. 41-7, 2012.

[189] D. Chemisana, "Building integrated concentrating photovoltaics: A review," Renewable and Sustainable Energy Reviews, vol. 15, pp. 603-11, 2011.

[190] S. Chandra, J. Doran, S. J. McCormack, M. Kennedy, and A. J. Chatten, "Enhanced quantum dot emission for luminescent solar concentrators using plasmonic interaction," Solar Energy Materials and Solar Cells, vol. 98, pp. 385-90, 2012.

[191] T. Saraidarov, V. Levchenko, A. Garbowska, P. Borowicz, and R. Reisfeld, "Non-selfabsorbing materials for luminescent solar concentrators (LSC)," Chemical Physics Letters, vol. 492, pp. 60-2, 2010. 
[192] K. Okamoto, S. Vyawahare, and A. Scherer, "Surface-plasmon enhanced bright emission from CdSe quantum-dot nanocrystals," J. Opt. Soc. Am. B, vol. 23, no. 8, pp. 1674-8, 2006.

[193] J. H. Song, T. Atay, S. Shi, H. Urabe, and A. V. Nurmikko, "Large enhancement of fluorescence efficiency from $\mathrm{CdSe} / \mathrm{ZnS}$ quantum dots induced by resonant coupling to spatially controlled surface plasmons," Nano Letters, vol. 5, no. 8, pp. 1557-61, 2005.

[194] E. Nihei, T. Ishigure, and Y. Koike, "High-bandwidth, graded-index polymer optical fiber for near-infrared use," Applied Optics, vol. 35, no. 36, pp. 7085-90, 1996.

[195] A. Nazarovsky. Fast CSV values export to file. MEX-based dlmwrite replacement for MATLAB. [Online]. Available: http://nazarovsky.ru/2014/08/22/fast-csv-valuesexport-to-file-mex-based-dlmwrite-replacement-for-matlab 


\section{Appendix A: List of Publications}

\section{Publications from this work}

1. D.L. Waldron, A. Preske, J.M. Zawodny, T. Krauss, and M.C. Gupta. "Lead selenide quantum dot polymer nanocomposites," Nanotechnology 26 (2015) 075705. doi:10.1088/0957-4484/26/7/075705

2. D.L. Waldron, A. Preske, J.M. Zawodny, T. Krauss, and M.C. Gupta. "Temperature dependent optical properties of lead selenide quantum dot polymer nanocomposites," (manuscript under internal review)

3. D.L. Waldron, A. Preske, J.M. Zawodny, T. Krauss, and M.C. Gupta. "Lead selenide quantum dot based luminescent solar concentrators," (manuscript under preparation)

4. E. Burke, P. Williams, S. Dehaven, M. Gupta, D. Waldron. "Scintillating quantum dots for imaging X-rays (SQDIX) for aircraft inspection," NASA 2014 Seedling Seminar (2014)

\section{Additional publications}

1. D.L. Waldron and L. Mullen. "Underwater optical ranging: A hybrid LIDAR-RADAR approach," OCEANS 2009, MTS/IEEE Biloxi (2009)

\section{ORCID ID}

For an up-to-date listing of publications, the author's ORCID ID is 0000-0001-9563-5575. 


\section{Appendix B: Ray Tracing Simulation}

\section{MATLAB Code}

The MATLAB source code for the LSC ray tracing simulations in chapter 3 is given below. In addition to various input data files, three MATLAB files are required: batch.m, Rtrace.m, and fresTMM.m.

The first, batch.m, initializes the user-set parameters of the simulation, such as LSC size, reflectivity of mirrors, etc., and calls the ray trace simulation function based on these parameters. It is named batch because it will run the simulation in a user-defined loop to see the change in a given parameter. It handles nearly all of the data output from the program, writing the data to various files on the hard drive.

batch.m also optionally calls the function frestMM to compute the reflection from the PV cell interface at all angles and wavelengths with a resolution of $1^{\circ}$ and $1 \mathrm{~nm}$. This needs to be done any time refractive indices or thicknesses of components in the dielectric stack on the PV cell are altered. frestMM is found in frestMM.m and uses Fresnel equations and the transfer matrix method. fresTMM.m was modified from TransferMatrix3.m [162].

The second, Rtrace.m contains two functions:

$$
\begin{aligned}
& \text { function }[\text { outputs }>\text { ] =Rtrace }(\text { args }>\text { ) } \\
& \text { function <output>=fresR (<args }>) \text {. }
\end{aligned}
$$

Rtrace is the main simulation. This will create input photons, inject them into the LSC, and follow their progress until they are output or lost. It is designed to run multiple photons in parallel for speed of execution. The number of simultaneous jobs allowed is determined by the command matlabpool ('open', n) where $\mathrm{n}$ is the number of workers in the pool. $\mathrm{n}$ cannot be more than the number of CPU cores available to the OS (hyper-threaded cores count towards this number). If this command is not run to set up parallel workers before the 
program is executed, it will run serially on a single CPU core. The Rtrace function makes use of the fresR function to calculate reflections from single dielectric interfaces as needed.

Note that batch.m optionally makes use of the mex_WriteMatrix function [195] to write large arrays to file much faster than the MATLAB dlmwrite function.

Under average conditions, the model will compute the result of $\sim 2 \times 10^{5}$ photons per CPU core per minute on an Intel Core $^{\mathrm{TM}}$ i7 $2.8 \mathrm{GHz} \mathrm{CPU}$.

\section{B.1 batch.m}

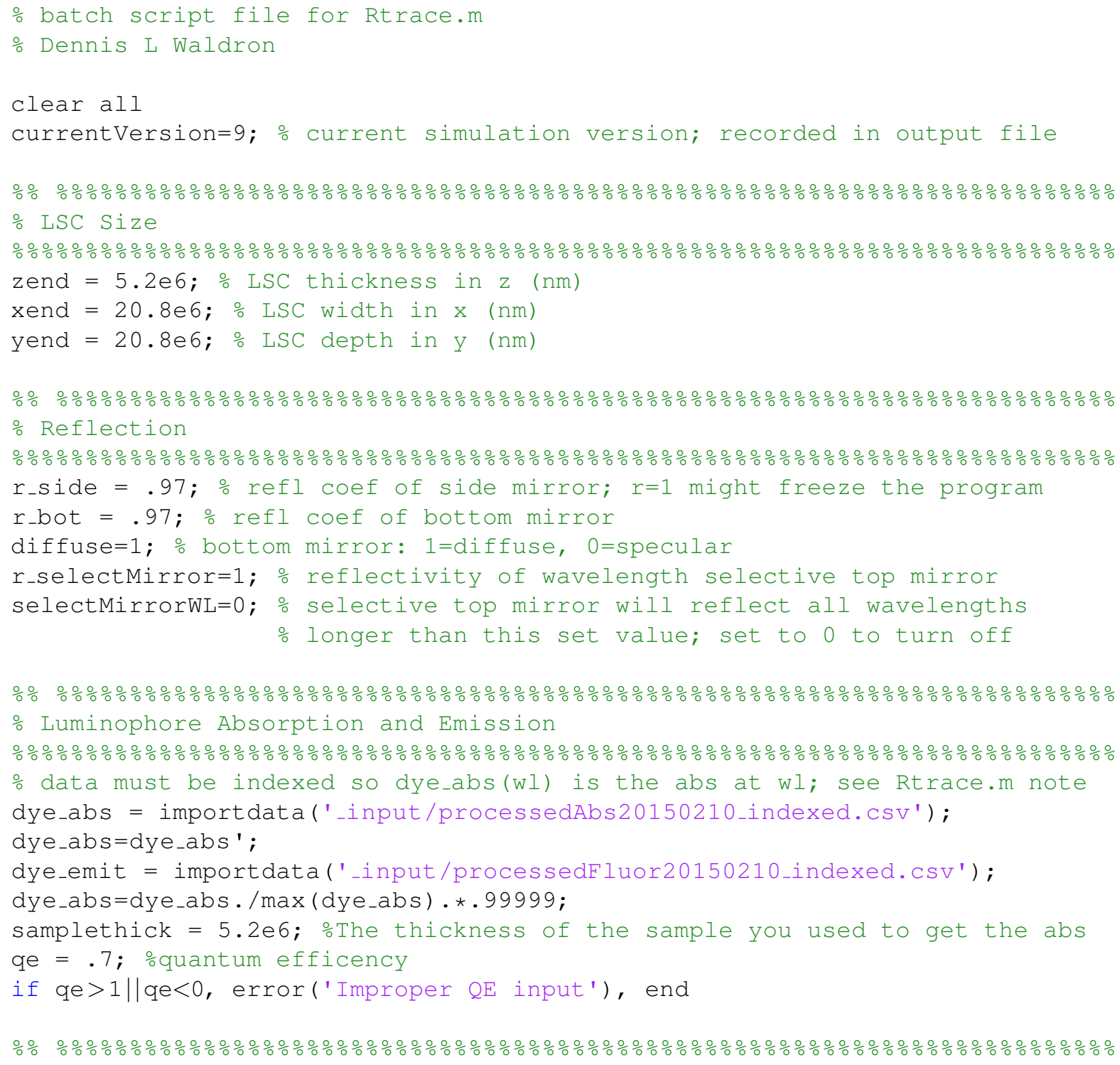


\% Matrix Absorption

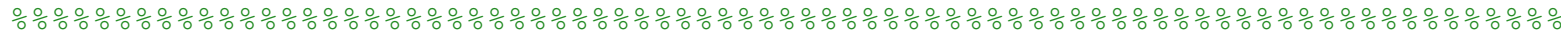
\% data must be indexed: see note in Rtrace.m matrixtype='plma'; \%plma, ab9093, clear, flat1, flat5

\% don't change below here in this section; loads data based on matrixType if strcmp (matrixType, 'plma') matrixAbs=importdata ('_input/matrixAbsPLMA_indexed.CSV') ; \% abs coef matrixThick=5.2e6;

\% convert abs coef from base 10 to base e tens $=$ repmat $(10$, size $($ matrixAbs $))$;

matrixAbs $=1$-tens.^ (-matrixAbs) ;

matrixAbs $=-\log (1-$ matrixAbs $)$;

n=importdata ( '_input/index/plmaedgma_indexed.cSV' ) ;

elseif strcmp (matrixtype, 'ab9093')

matrixAbs=importdata (' _input/matrixAbsAB9093_indexed.CSV'); \% abs coef matrixThick=5.2e6;

\% convert abs coef from base 10 to base e

tens $=$ repmat $(10$, size (matrixAbs) ) ;

matrixAbs $=1$-tens.^ (-matrixAbs) ;

matrixAbs $=-\log (1-$ matrixAbs $)$;

n=importdata ('_input/index/ab9093_indexed.CSV') ;

elseif strcmp (matrixType, 'clear')

matrixAbs=zeros $(1100,1)$;

matrixThick=10e6;

n=importdata ( '_input/index/plmaedgma_indexed.csv' ) ;

elseif strcmp (matrixtype, 'flatl') \% 1\% abs per cm for all wavelength

matrixAbs=ones $(1100,1) \star-\log (1-.01)$;

matrixThick $=10$ e6;

n=importdata ( ' -input/index/onePointFive_indexed.csv' );

elseif strcmp (matrixtype, 'flat5') \% 5\% abs per cm for all wavelength

matrixAbs=ones $(1100,1) \star-\log (1-.05)$;

matrixThick=10e6;

n=importdata (' _input/index/onePointFive_indexed.Csv') ;

else

error('Improper matrix type input')

end

clear tens

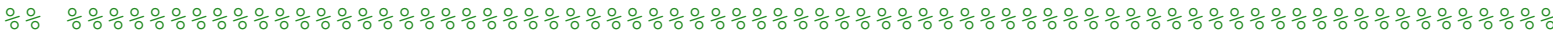
응 Source Type

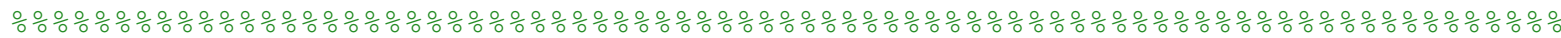

src_type='osl1'; \% options: osl1, diffuselaser, laser,

\% sunglobaltilt, sunglobaltilt1100

Wl_srC=532; $\%$ source WL ( $\mathrm{nm})$ for diffuseLaser and laser; ignored otherwise

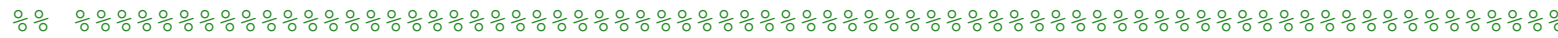
응 Other Parameters

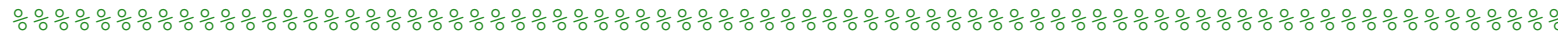

output_filter $=0$; $\frac{\circ}{\circ}$ If 1 , then only light that has been absorbed and o re-emitted by the dye will be accepted as output

$\mathrm{N}=1 ;$; Scaling factor for number of dye particles per unit volume (arb) ptotal $=2 e 6 ;$ Increase until you get convergance; le6 usually plenty runName='qdDefaultCase'; \% simulation run name for naming output files delete(['_output/' runName '_absgraph.csv']) \% delete: first write appends 


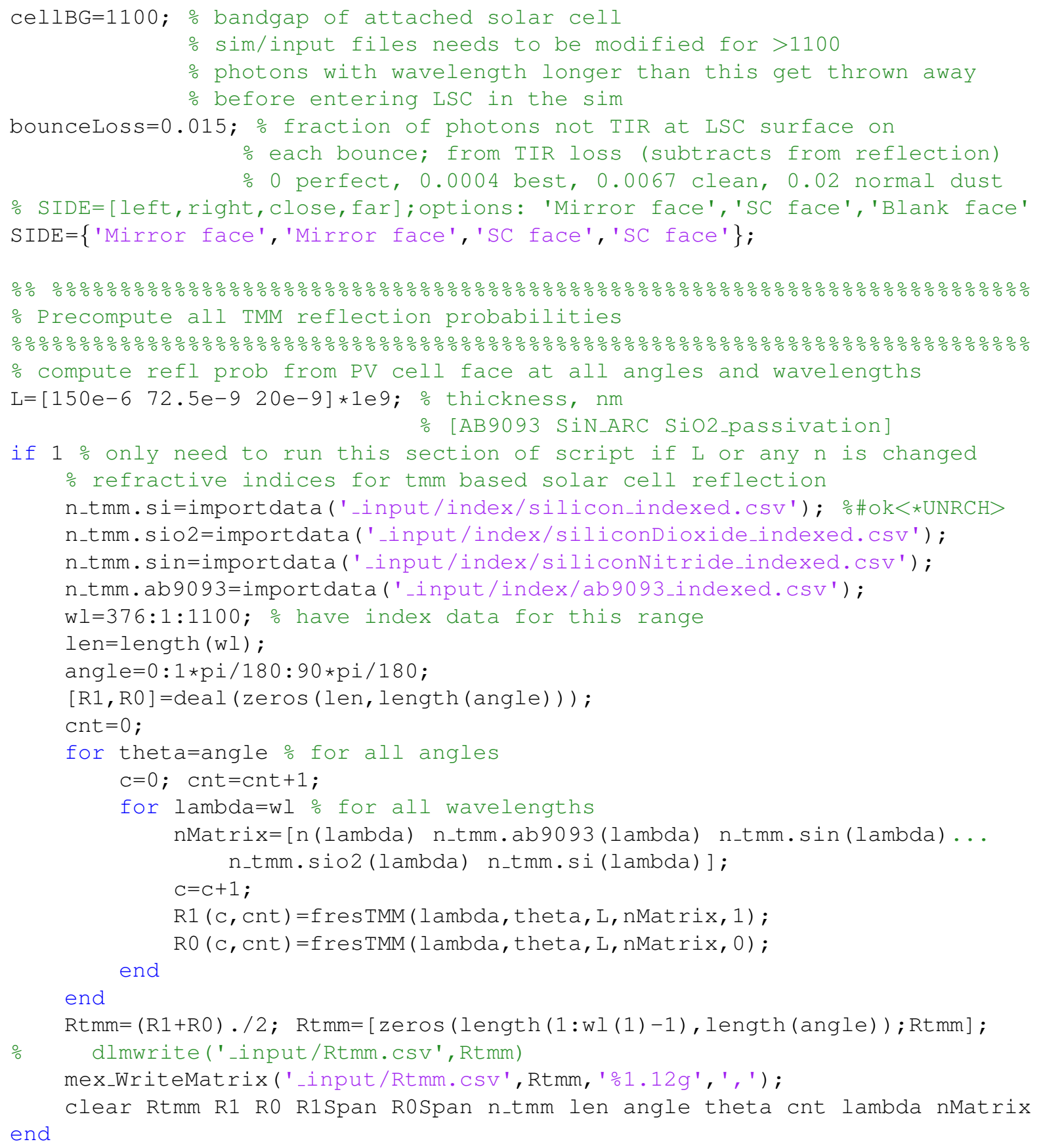


tic $\%$ start timer

$\mathrm{C}=\mathrm{C}+1$;

[eff(c), SA(c), totTrav(c), poutTrav(c), LEmatrix(c, :), Loss (c, :),... $\operatorname{emWL}(:, c)$, emAngle $(:, c), \operatorname{pwl}] \ldots$

= Rtrace (runName, $n, N$, zend, xend, yend, qe, r_bot, r_side, diffuse,... ptotal, samplethick, output_filter, dye_abs, dye_emit, src_type,... selectMirrorWL, r_selectMirror, matrixAbs, matrixThick,... cellBG, wl_src, bounceloss, SIDE);

toc $\%$ stop timer and display elapsed time

end

o matlabpool close; uncomment if matlabpool not manually set

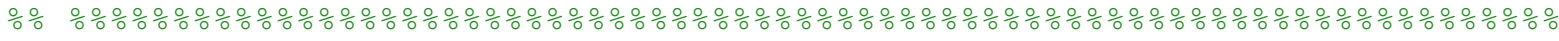
\% Output

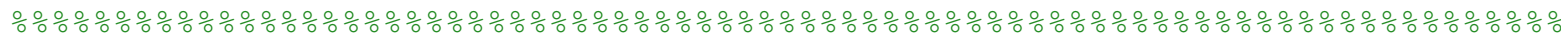
estEQE=.8; measFF=.7; belowBand=.631; measVoc=.5485; \% meas or est PV cell if strcmp (src_type, 'sunglobaliilt')

belowBand=1; end $\frac{\circ}{\circ}$ full solar spectrum, belowband already accounted for

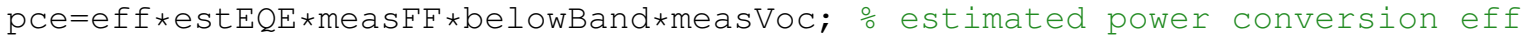
$\max (e f f), \max (p c e)$ o print maximum efficiencies to console

$\div$ plot

figure(1); plot (ind_var, eff, 'bo-') ;

hold on; plot(ind_var,pce, 'ro-'); hold off

\% txt file of parameters and results $===============================$ id $=$ fopen ( ['_output/' runName '.txt'], 'w');

fprintf (id, 'Concentration teff $\backslash r \backslash n$ ');

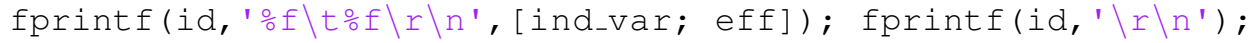

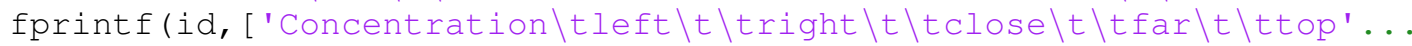

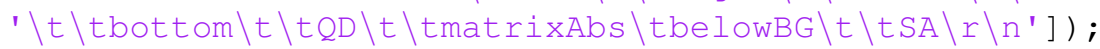

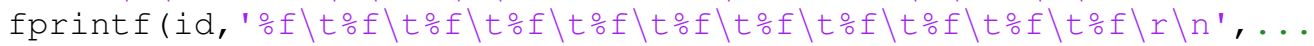
[ind_var', LEmatrix, SA']'); fprintf(id, '\r\n');

fprintf(id, ['Concentration\tlossConeSA ttlossCone tunabsorbed ttFSR' ...

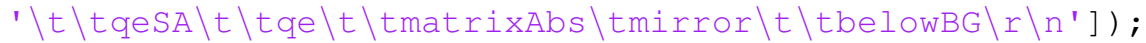

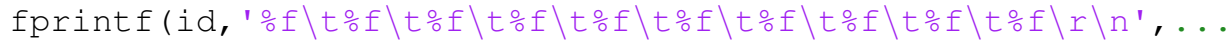
[ind_var',Loss]'); fprintf(id, '\r\n');

fprintf(id, 'Current Simulation Version= od \r\n', currentVersion);

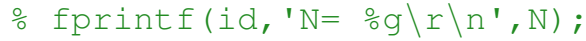

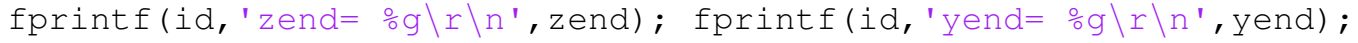

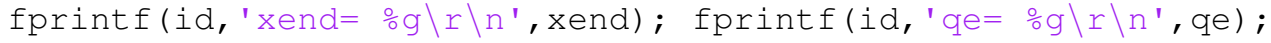

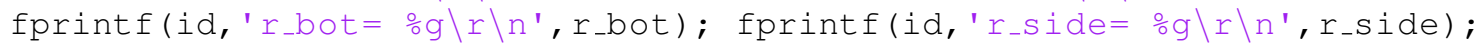

fprintf (id, 'r_selectMirror = $\frac{\mathrm{g}}{\mathrm{f}} \backslash \mathrm{r} \backslash \mathrm{n}$ ', r_selectMirror);

fprintf (id, 'selectMirrorWL $=\circ g \backslash r \backslash n$ ', selectMirrorWL);

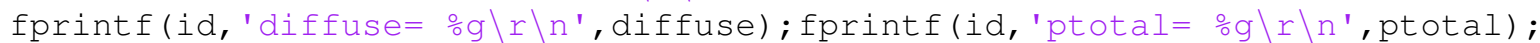

fprintf (id, 'samplethick= og $\backslash r \backslash n$ ', samplethick) ;

fprintf(id, 'output_filter $=\circ g \backslash r \backslash n$ ', output_filter);

fprintf (id, 'src_type= os $\backslash r \backslash n$ ', src_type) ;

fprintf (id, 'matrixType $=\% s \backslash r \backslash n$ ', matrixType) ;

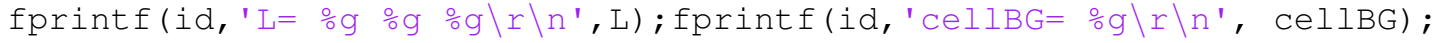

fprintf(id, 'wl_src $=\circ g \backslash r \backslash n$ ', wl_src) ;

fprintf (id, 'bounceLoss= og $\backslash r \backslash n$ ', bounceLoss) ;

side $=[\operatorname{SIDE}\{1\}$ ', ' $\operatorname{SIDE}\{2\}$ ', ' $\operatorname{SIDE}\{3\}$ ', ' $\operatorname{SIDE}\{4\}]$;

fprintf(id, 'SIDE: os $\backslash r \backslash n^{\prime}$, side); fprintf(id, 'Matrix $\left.n=\backslash r \backslash n^{\prime}\right)$; 


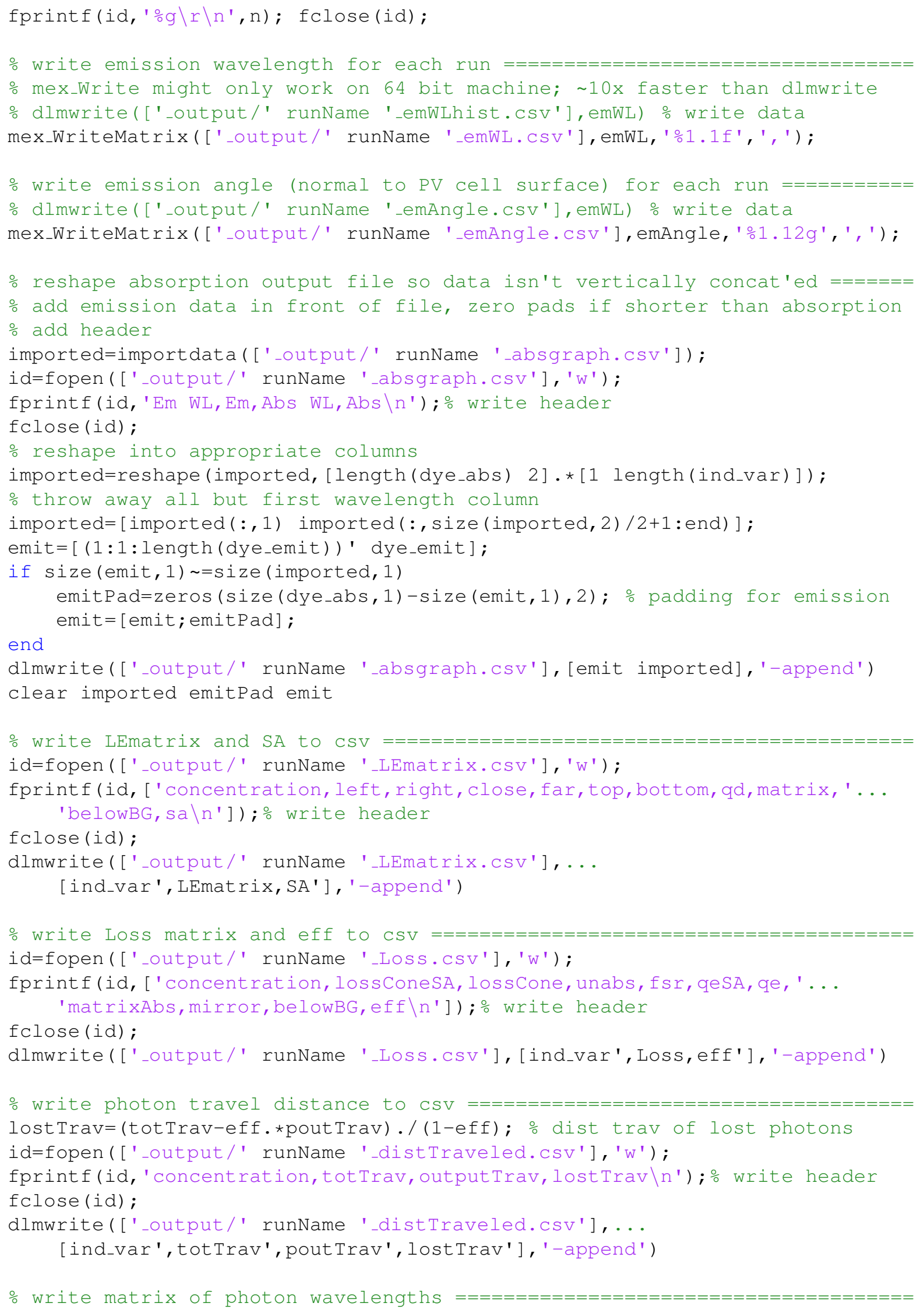


\% dlmwrite( ['_output/' runName '_sourceWavelength.csv'], pwl)

mex_WriteMatrix(['_output/' runName '_sourceWavelength.csv'],pwl,...

'⒈ 1 f', ', ');

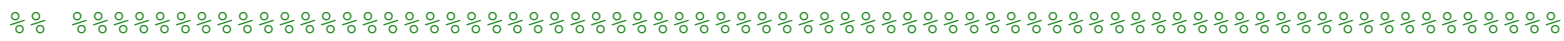
응 Done

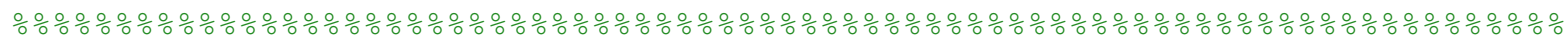

'DONE DONE DONE DONE' \%\#OK<NOPTS> output alert to console

load Handel \% load sound clip

$\mathrm{y}=\mathrm{y}(1:$ floor $($ length $(\mathrm{y}) / 7)) ;$ o shorten clip

$\mathrm{y}=\mathrm{y} /(\max (\max (\mathrm{y}), \min (\mathrm{y}) \star-1)) \star 0.2 ;$ 을 reduce volume

sound $(y, F s) \div$ play alert that simulation is done

\section{B.2 Rtrace.m}

\% Run from batch.m (version history in batch.m)

\% Dennis L Waldron and Craig Ungaro

function [eff, SA_count, tot_trav, pouttrav, LEmatrix, Loss, emWL, emAngle, pwl] =...

Rtrace (runName, $n, N$, zend, xend, yend, qe, r_bot, r_side, diffuse,...

ptotal, samplethick, output_filter,dye_abs, dye_emit, src_type,...

selectMirrorWL, r_selectMirror, matrixAbs, matrixThick, ...

cellBG, wl_src, bounceLoss, SIDE)

을 - LSC raytracing model (3D)

$\div\{$

All distances and wavelengths in $\mathrm{nm}$

Inputs:

runName string, for naming output files

n indexed array of matrix material refractive index

$\mathrm{N} \quad$ concentration of luminophore relative to input conc.

zend thickness of simulated LSC, nm

xend $\quad x$ length of simulated LSC, nm

yend $\quad y$ length of simulated LSC, nm

qe quantum efficiency of luminophore, range [0 1 ]

r_bot reflectivity of bottom mirror, range [0 1]

r_side reflectivity of side mirror, range [ $\left.\begin{array}{ll}0 & 1\end{array}\right]$

diffuse bottom mirror: 1 = diffuse (Lambertian), 0 = specular

ptotal total number of photons to simulate

samplethick thickness of sample used to measure dye_abs

output_filter 0 = no effect, 1 = photon will only be counted as output if it has been abs and emit at least once

dye_abs

dye_emit

src_type

indexed array of absorption probability, range [ 01 1)

indexed array of relative fluor spectra, any range

string, type of input source:

osl1: Thorlabs osL1 halogen fiber illuminator, distrubuted evenly across sample

laser: $532 \mathrm{~nm}$, center of LSC w/ Gaussian spread sunglobalTilt: ATSM 1.5 sunlight source, distrubuted evenly across sample sunglobalTilt1100: ATSM 1.5 sunlight source, only below $1100 \mathrm{~nm}$, distrubuted evenly across sample selectMirrorWL cut-on wavelength of selective top mirror, 0 for off 


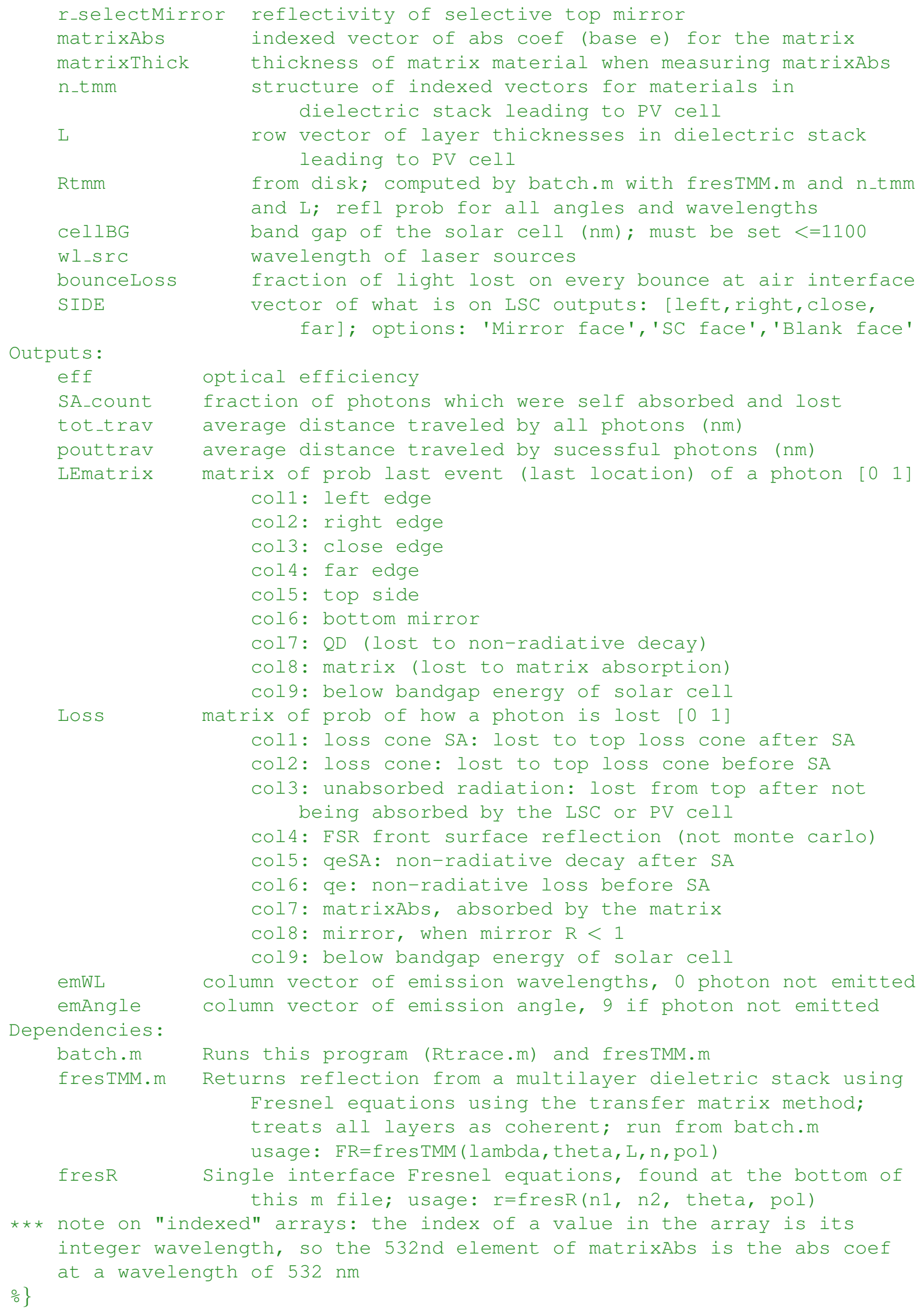




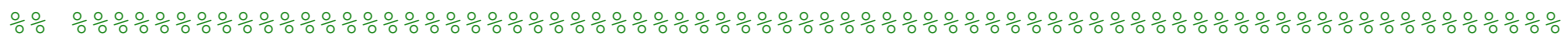
Misc. variable initilization

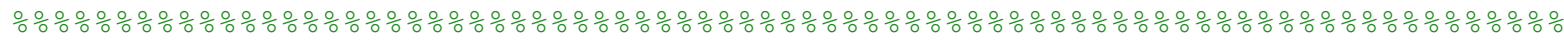
Rtmm=importdata ('_input/Rtmm.CSV'); \% precomputed PV cell face reflection LEmatrix =zeros $(1,9)$;

Loss $=$ zeros $(1,9)$;

emWL=zeros (ptotal, 1$)$; $\frac{\circ}{\circ}$ init to impossible value for later separation

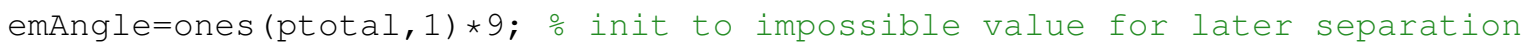
SA_count $=0$; pouttrav $=0$; tot_trav $=0$;

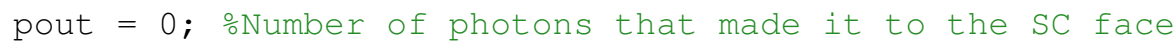
if $\max \left(\right.$ dye_abs $\left._{-}=1\right)$

error(['Dye absorption must be in units of pout/pin (max should be '... 'less than 1). If your max is 1 change it to.9999'])

end

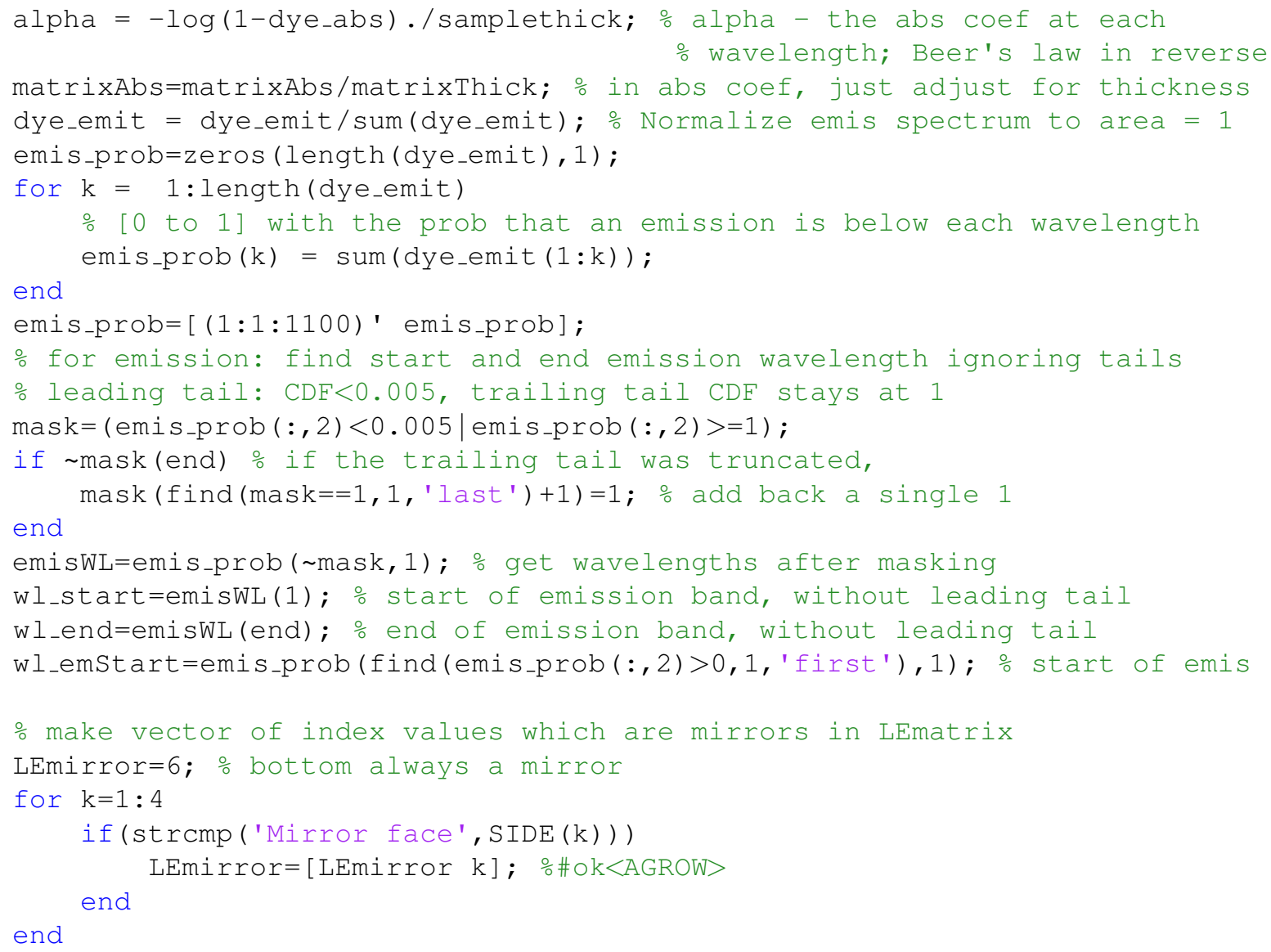




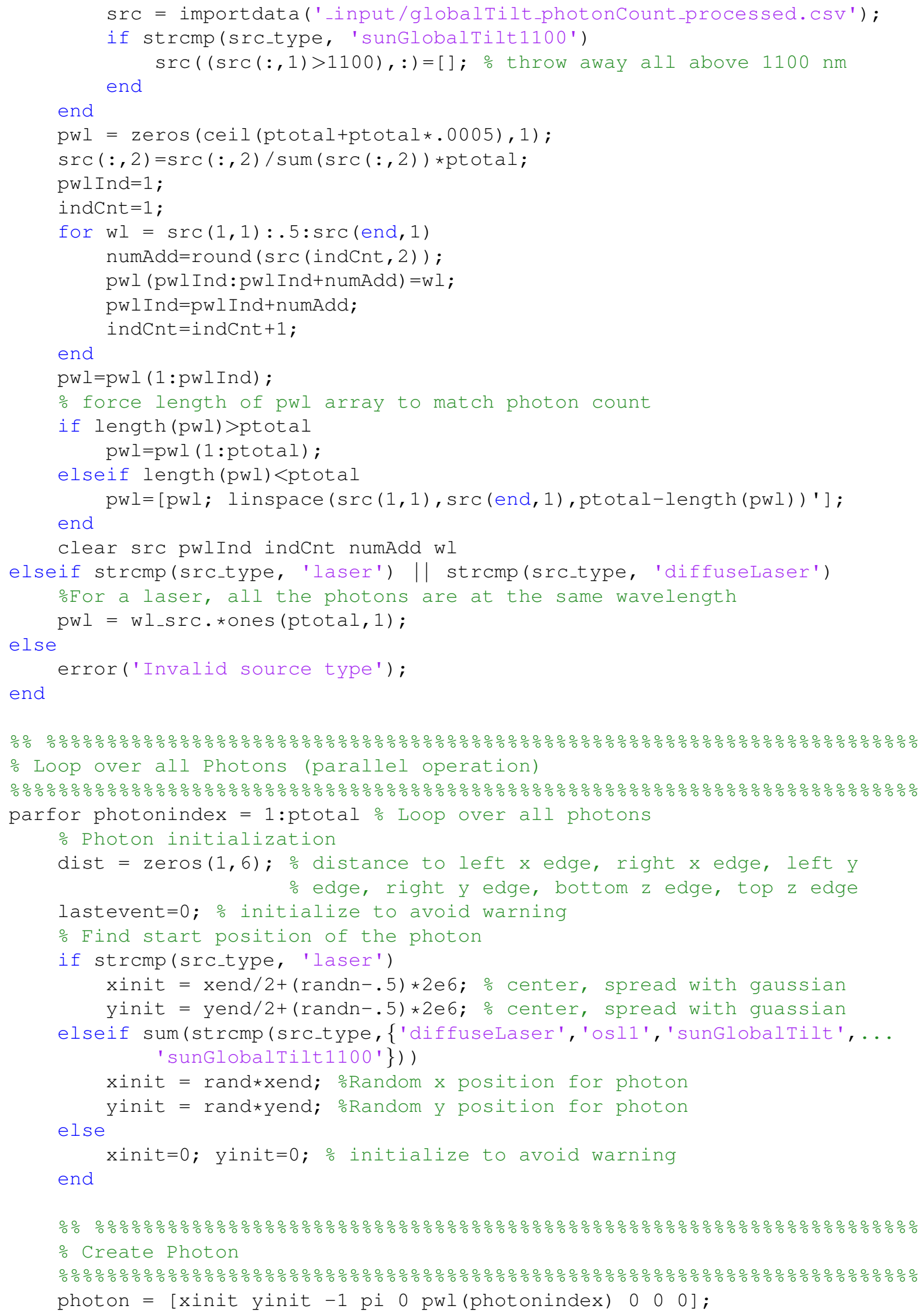




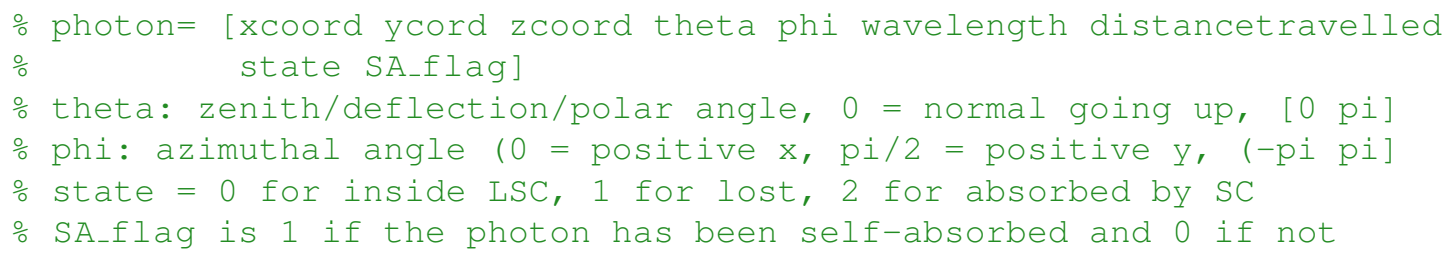




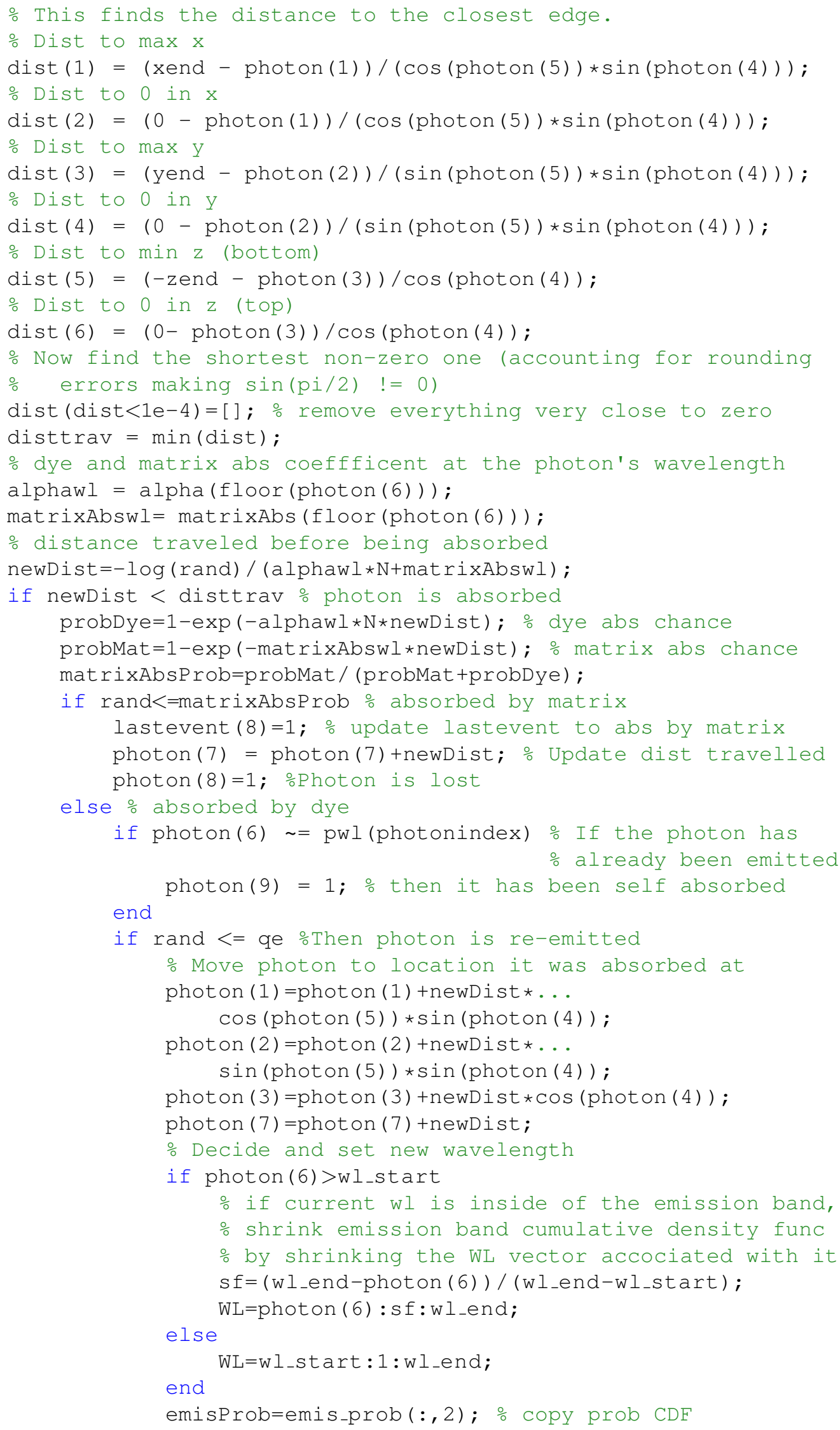




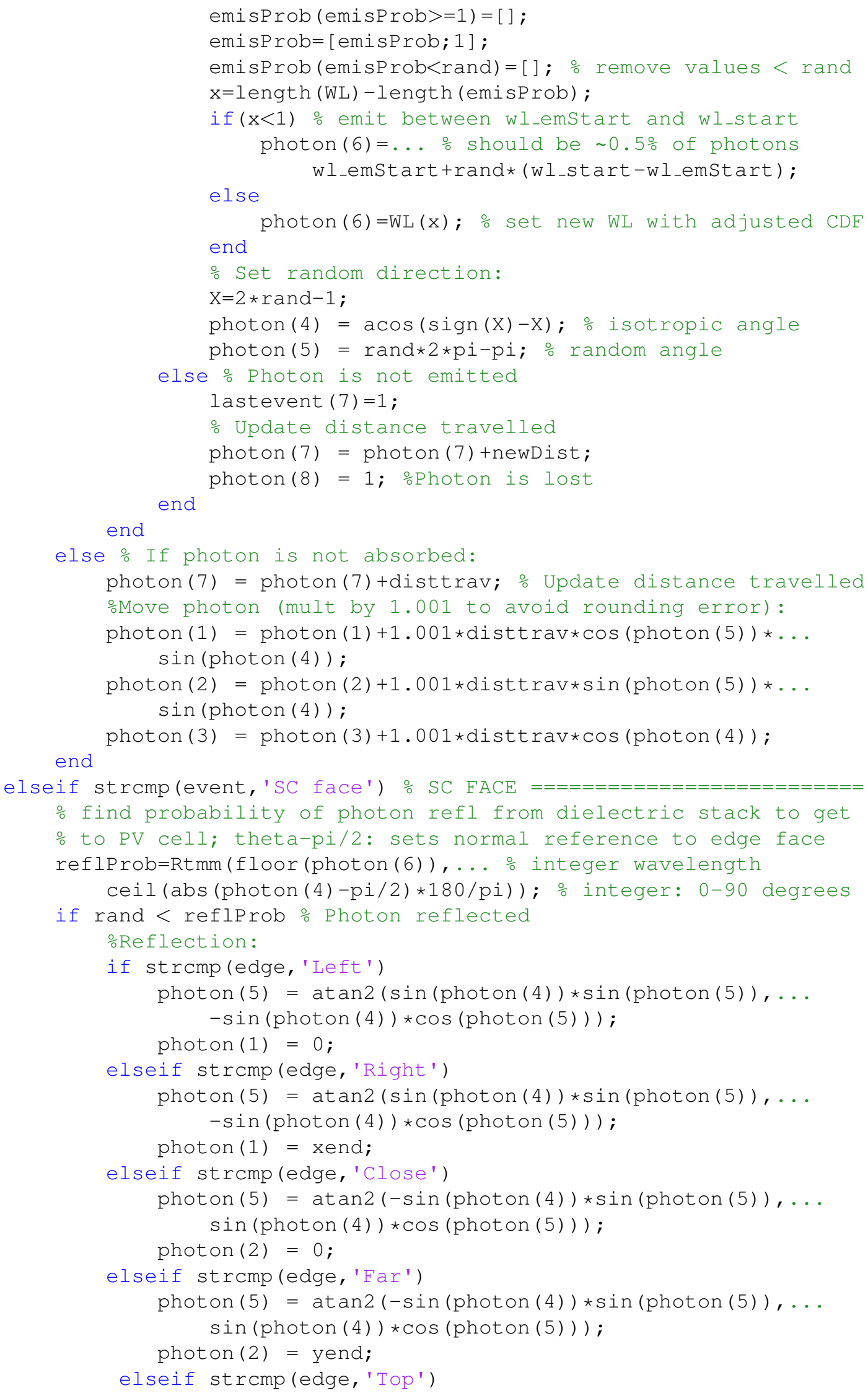




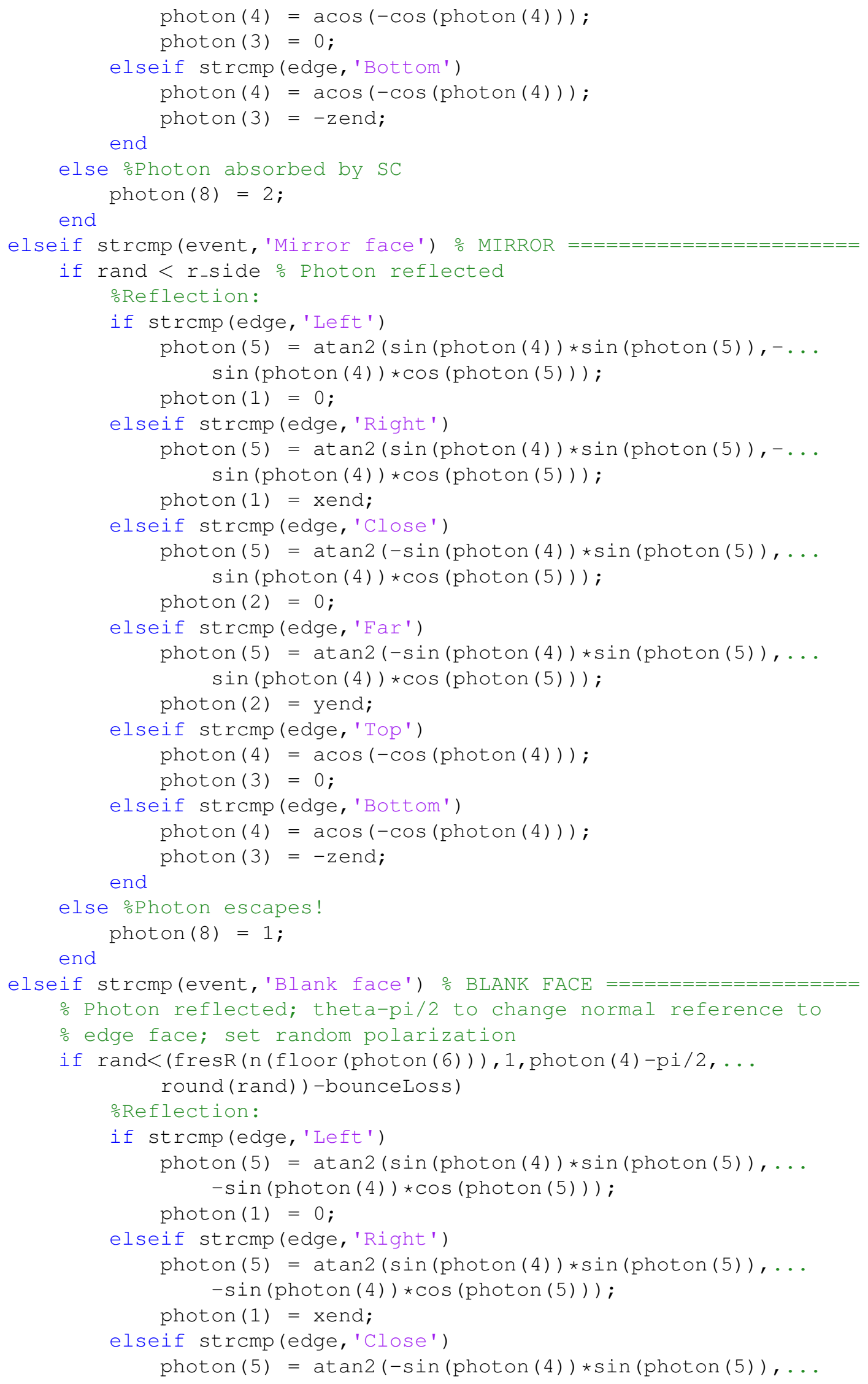




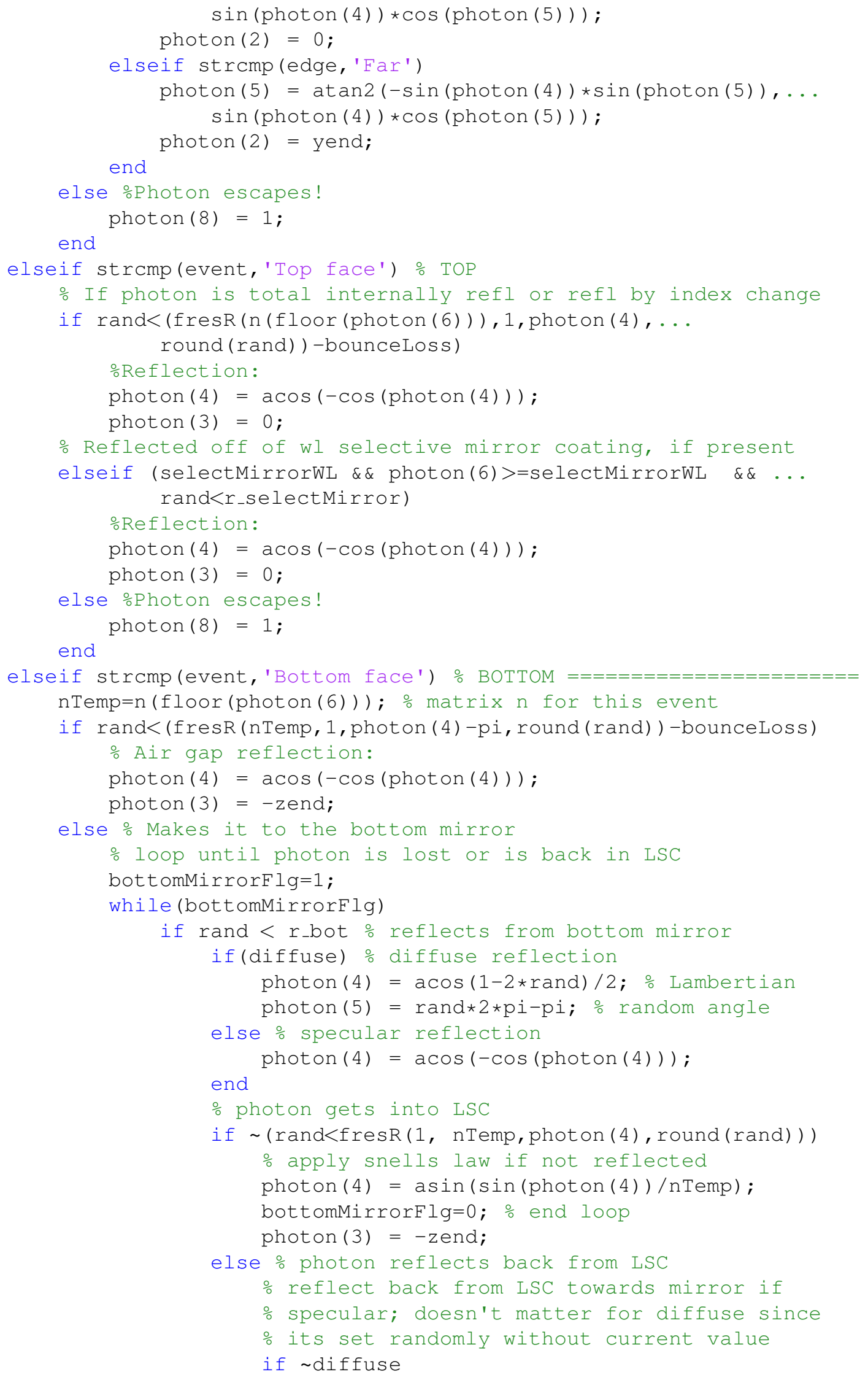




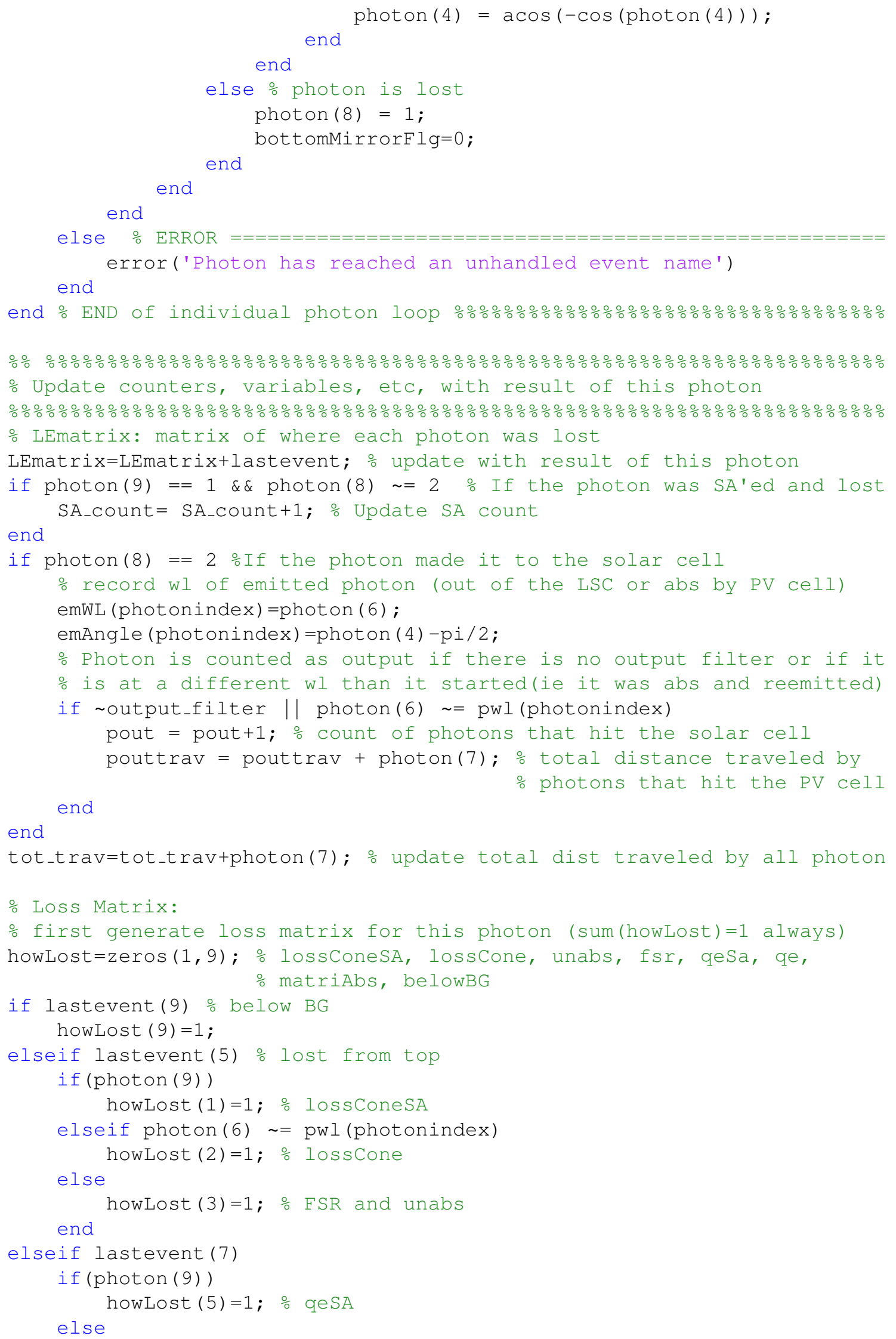




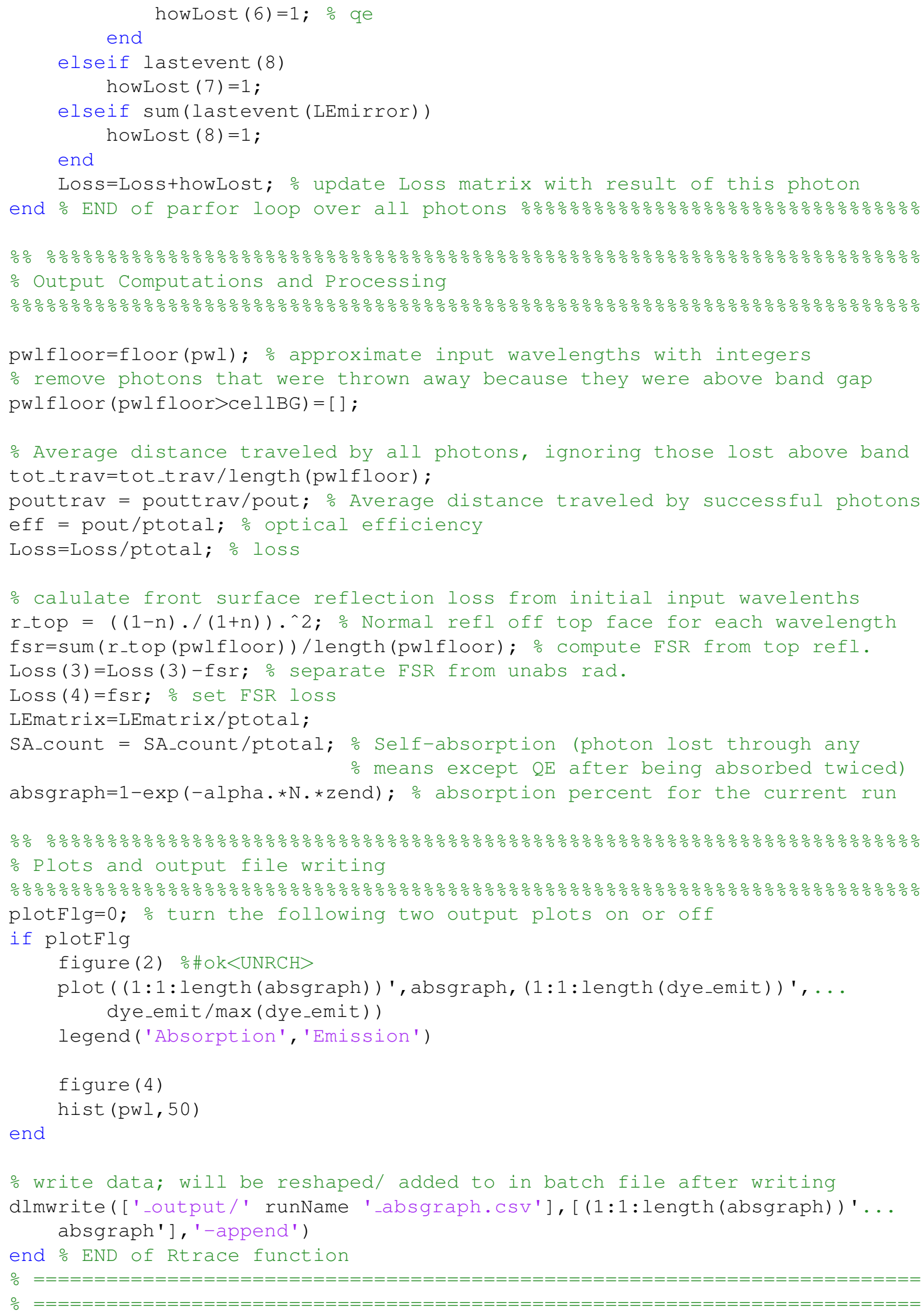




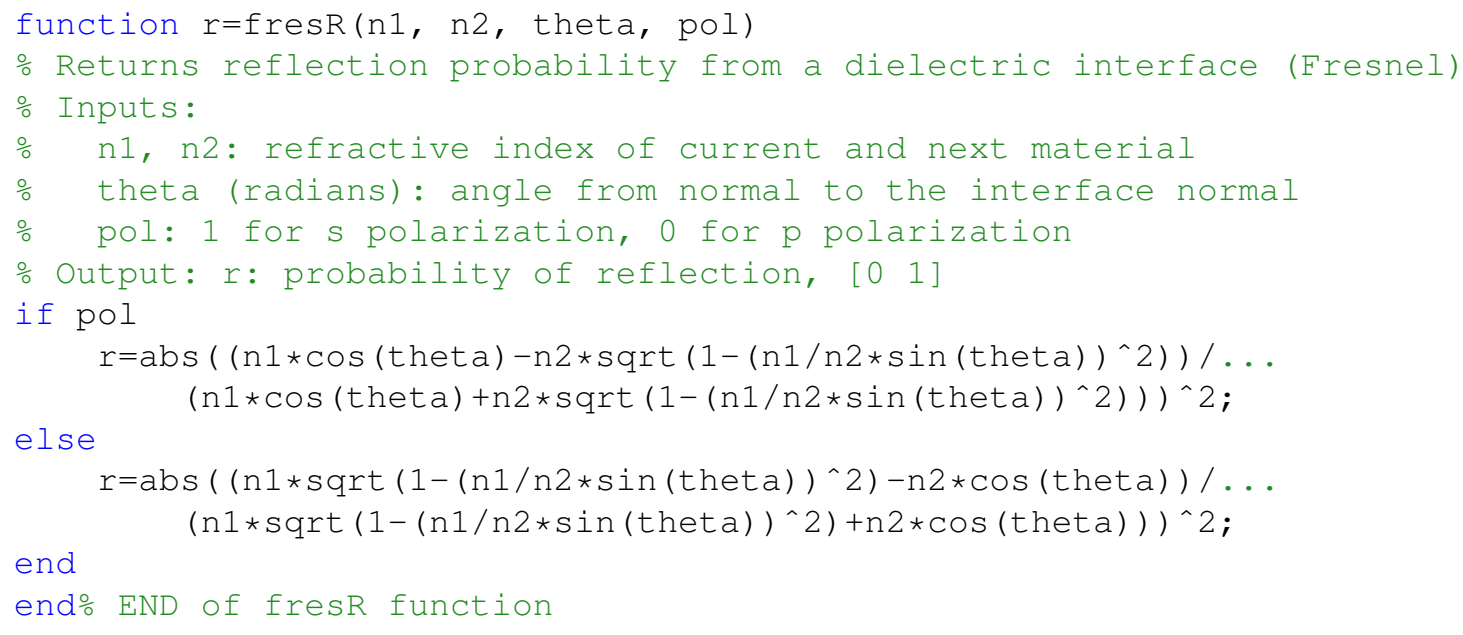

\section{B.3 fresTMM.m}

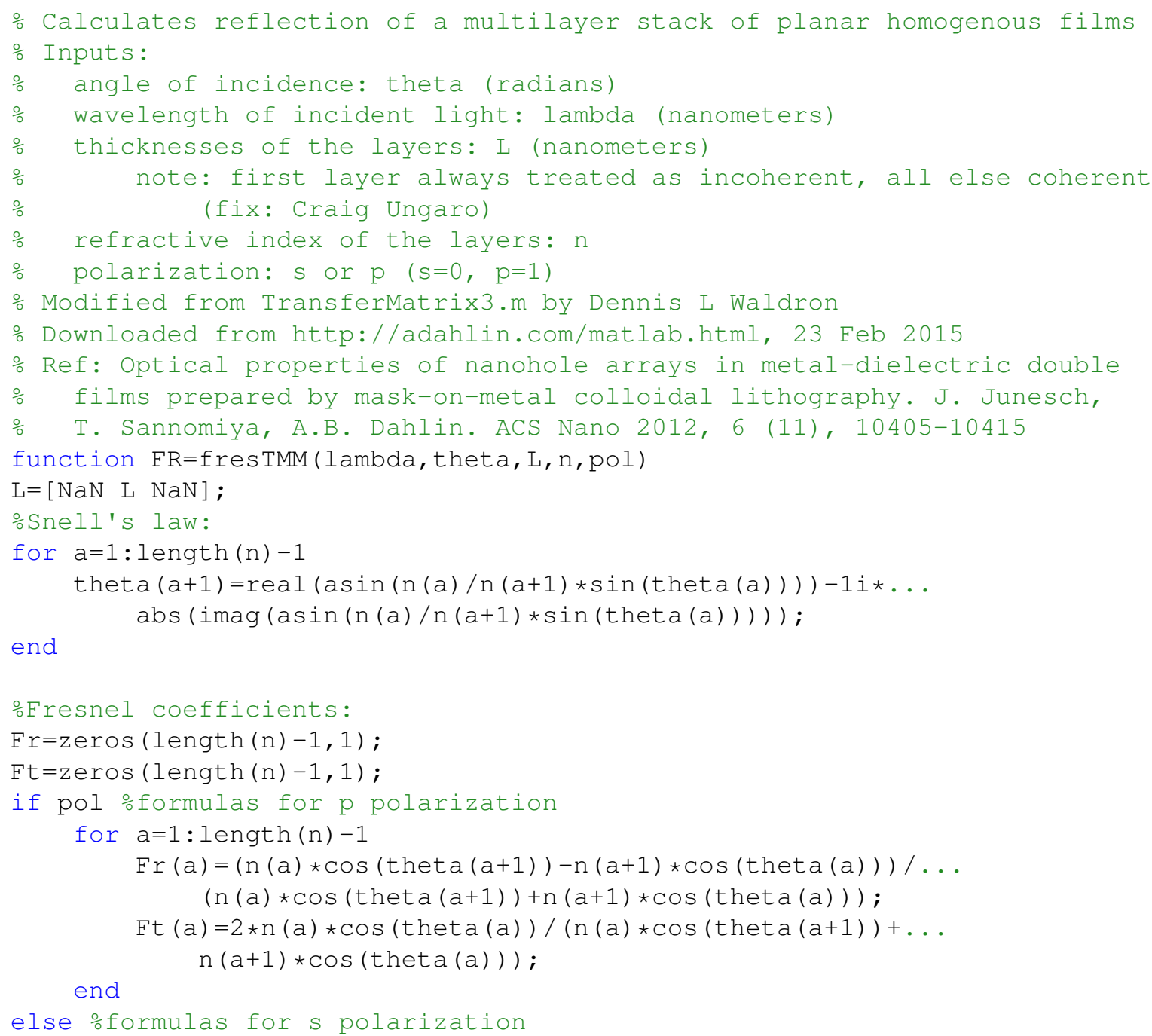


for $a=1:$ length $(n)-1$

$\operatorname{Fr}(a)=(n(a) * \cos ($ theta $(a))-n(a+1) * \cos ($ theta $(a+1))) / \ldots$

$(\mathrm{n}(\mathrm{a}) \star \cos ($ theta $(\mathrm{a}))+\mathrm{n}(\mathrm{a}+1) \star \cos ($ theta $(\mathrm{a}+1)))$;

$\mathrm{Ft}(\mathrm{a})=2 \star \mathrm{n}(\mathrm{a}) \star \cos ($ theta $(\mathrm{a})) /(\mathrm{n}(\mathrm{a}) \star \cos ($ theta $(\mathrm{a}))+\ldots$

$\mathrm{n}(\mathrm{a}+1) * \cos ($ theta $(\mathrm{a}+1)))$;

end

end

- phase shift factors:

delta=zeros (length $(\mathrm{n})-2,1)$;

for $\mathrm{a}=1$ : length $(\mathrm{n})-2$

$\operatorname{delta}(\mathrm{a})=2 * \mathrm{p} i * \mathrm{~L}(\mathrm{a}+1) / \operatorname{lambda} * \mathrm{n}(\mathrm{a}+1) * \cos ($ thet $\mathrm{a}(\mathrm{a}+1))$;

end

\% build up transfer matrix:

\% First, the infinite and incoherent layers

$\mathrm{a}=1$;

$\mathrm{T}_{-} i=1 / \operatorname{Ft}(\mathrm{a}) \star[1, \operatorname{Fr}(\mathrm{a}) ; \operatorname{Fr}(\mathrm{a}), 1] *[\exp (-1 i \star \operatorname{delta}(\mathrm{a})), 0 ; 0, \exp (1 \mathrm{i} * \operatorname{delta}(\mathrm{a}))]$;

$r_{-} T_{-} i=T_{-} i(2,1) / T_{-} i(1,1)$; $\frac{\circ}{0}$ complex reflection coefficient of $T_{-} i$

$t_{-} \mathrm{T}_{-} i=1 / \mathrm{T}_{-} i(1,1)$; $\frac{\circ}{0}$ complex transmission coefficient of $\mathrm{T}_{-} i$

r_p_T_i=-T_i $(1,2) / T_{-} i(1,1)$; o complex reflection coefficient of $T_{-} i$

\% complex transmission coefficient of $T_{-} i$

$t_{-} P_{-} T_{-} i=\left(T_{-} i(1,1) * T_{-} i(2,2)-T_{-} i(1,2) * T_{-} i(2,1)\right) / T_{-} i(1,1)$;

\% transfer matrix for the incoherent layer

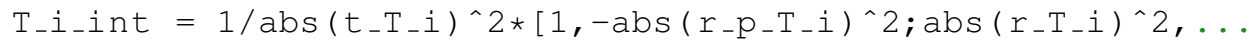

$\left.\left(a b s\left(t_{-} T_{-} i \star t_{-} p_{-} T_{-} i\right)^{\wedge} 2-a_{b s}\left(r_{-} T_{-} i \star r_{-} p_{-} T_{-} i\right)^{\wedge} 2\right)\right] ;$

$\mathrm{T}_{-} \mathrm{C}=[1,0 ; 0,1] ; \%$ start $\mathrm{w} / \mathrm{m}$ ity matrix for coherent stack transfer matrix

o Coherent portion:

for $\mathrm{a}=2$ : length $(\mathrm{n})-2$

$\mathrm{T}_{-} \mathrm{C}=\mathrm{T}_{-} \mathrm{C} \star 1 / \mathrm{Ft}(\mathrm{a}) \star[1, \operatorname{Fr}(\mathrm{a}) ; \operatorname{Fr}(\mathrm{a}), 1] *[\exp (-1 \mathrm{i} \star \operatorname{delta}(\mathrm{a})), 0 ; \ldots$

$0, \exp (1 i \star \operatorname{delta}(\mathrm{a}))]$;

end

\%Last (infinite) layer

$\mathrm{T}_{-} \mathrm{C}=\mathrm{T}_{-} \mathrm{C} * 1 / \mathrm{Ft}($ length $(\mathrm{n})-1) *[1, \operatorname{Fr}($ length $(\mathrm{n})-1) ; \mathrm{Fr}($ length $(\mathrm{n})-1), 1]$;

$r_{-} T_{-} C=T_{-} C(2,1) / T_{-} C(1,1) ; \%$ complex reflection coefficient of $T_{-} C$

$t_{-} T_{-} C=1 / T_{-} C(1,1) ; \%$ complex transmission coefficient of $T_{-} C$

$r_{-} p_{-} T_{-} C=-T_{-} C(1,2) / T_{-} C(1,1) ; \%$ complex reflection coefficient of $T_{-} C$

o complex transmission coefficient of $T_{-} C$

$t_{-} \mathrm{P}_{-} \mathrm{T}_{-} \mathrm{C}=\left(\mathrm{T}_{-} \mathrm{c}(1,1) \star \mathrm{T}_{-} \mathrm{C}(2,2)-\mathrm{T}_{-} \mathrm{c}(1,2) \star \mathrm{T}_{-} \mathrm{C}(2,1)\right) / \mathrm{T}_{-} \mathrm{C}(1,1)$;

$\mathrm{T}_{-} \mathrm{c}_{-}$int $=1 / \mathrm{abs}\left(\mathrm{t}_{-} \mathrm{T}_{-} \mathrm{c}\right)^{\wedge} 2 *\left[1,-\mathrm{abs}\left(\mathrm{r}_{-} \mathrm{p}_{-} \mathrm{T}_{-} \mathrm{c}\right)^{\wedge} 2 ; \mathrm{abs}\left(\mathrm{r}_{-} \mathrm{T}_{-} \mathrm{c}\right)^{\wedge} 2, \ldots\right.$

$\left.\left(a b s\left(t_{-} T_{-} c * t_{-} p_{-} T_{-} c\right)^{\wedge} 2-a b s\left(r_{-} T_{-} c * r_{-} P_{-} T_{-} c\right)^{\wedge} 2\right)\right] ;$

$\mathrm{M}=\mathrm{T}_{-} i_{-}$int $* \mathrm{~T}_{-} \mathrm{C}_{-}$int; $\%$ Total transfer matrix

\% total Fresnel coefficients:

if length $(n)==2$ o special case of single interface:

Frtot $=\operatorname{Fr}(1) ;$

else

Frtot $=\mathrm{M}(2,1) / \mathrm{M}(1,1)$;

end

$\mathrm{FR}=($ abs (Frtot)) ; o total Fresnel coefficients in intensity

end 